\title{
The Role Of Hypoxia and Vascular Growth Factors in Experimental Atherosclerosis
}

Citation for published version (APA):

Theelen, T. L. (2016). The Role Of Hypoxia and Vascular Growth Factors in Experimental Atherosclerosis. [Doctoral Thesis, Maastricht University]. Proefschriftmaken.nl || Uitgeverij BOXPress.

https://doi.org/10.26481/dis.20160520tt

Document status and date:

Published: 01/01/2016

DOI:

10.26481/dis.20160520tt

Document Version:

Publisher's PDF, also known as Version of record

\section{Please check the document version of this publication:}

- A submitted manuscript is the version of the article upon submission and before peer-review. There can be important differences between the submitted version and the official published version of record.

People interested in the research are advised to contact the author for the final version of the publication, or visit the DOI to the publisher's website.

- The final author version and the galley proof are versions of the publication after peer review.

- The final published version features the final layout of the paper including the volume, issue and page numbers.

Link to publication

\footnotetext{
General rights rights.

- You may freely distribute the URL identifying the publication in the public portal. please follow below link for the End User Agreement:

www.umlib.nl/taverne-license

Take down policy

If you believe that this document breaches copyright please contact us at:

repository@maastrichtuniversity.nl

providing details and we will investigate your claim.
}

Copyright and moral rights for the publications made accessible in the public portal are retained by the authors and/or other copyright owners and it is a condition of accessing publications that users recognise and abide by the legal requirements associated with these

- Users may download and print one copy of any publication from the public portal for the purpose of private study or research.

- You may not further distribute the material or use it for any profit-making activity or commercial gain

If the publication is distributed under the terms of Article $25 \mathrm{fa}$ of the Dutch Copyright Act, indicated by the "Taverne" license above, 


\section{The Role of Hypoxia and Vascular Growth Factors in Experimental Atherosclerosis}


C Thomas L. Theelen, Maastricht 2016

All rights reserved. No part of this book may be reproduced, stored in a retrieval system of any nature, or transmitted in any form or by any means (electronic, mechanical, photocopying, recording or otherwise) without prior written permission of the author, or when appropriate, by the publishers of the publications.

ISBN:

978-94-6295-480-9

Author:

Thomas L. Theelen

Layout \& figure design: Thomas L. Theelen

Cover art and design: Volker Zerbe (vz@volker-zerbe.de)

Published by:

Uitgeverij BOXPress, 's Hertogenbosch

Printed by:

Proefschriftmaken.nl || Uitgeverij BOXPress

Fundedby
the dutch heartfoundation Financial support by the Dutch Heart Foundation for the publication

I. Hartstichting of this thesis is gratefully acknowledged.

Financial support by SSF, ABN AMRO for the publication of this thesis is gratefully acknowledged. 


\title{
The Role of Hypoxia and Vascular Growth Factors in Experimental Atherosclerosis
}

\author{
DISSERTATION \\ to obtain the degree of Doctor at Maastricht University, \\ on the authority of Rector Magnificus, Prof. dr. L.L.G. Soete, \\ in accordance with the decision of the Board of Deans, \\ to be defended in public
}

on Friday, May 20 2016 at 10.00 o'clock

by

Thomas Lukas Theelen

born on October $29^{\text {th }} 1985$ in Mönchengladbach, Germany 


\section{Supervisors}

Prof. dr. Mat J.A.P. Daemen

Prof. dr. Erik A.L. Biessen

\section{Co-supervisor}

Dr. Judith C. Sluimer

\section{Assessment committee}

Prof. dr. Tilman M. Hackeng (chairman)

Prof. dr. Jürgen Bernhagen, Klinikum der Universität München, Germany

Dr. Paula A. da Costa Martins

Prof. dr. Robert J. van Oostenbrugge

Prof. dr. Anton Jan van Zonneveld, Leiden University Medical Center 


\section{Contents}

$\begin{array}{lll}\text { Chapter } 1 & \text { General introduction and outline of the thesis }\end{array}$

Chapter $2 \quad$ Reversal of hypoxia in murine atherosclerosis prevents 27

necrotic core expansion by enhancing efferocytosis

Chapter $3 \quad$ Myeloid prolyl hydroxylase domain-containing protein 2

53

deficiency leads to larger but more stable atherosclerotic

plaques in mice

Chapter $4 \quad$ Deficiency of platelet derived growth factor B retention motif

is associated with increased atherosclerotic plaque fibrosis

and size

Chapter $5 \quad$ Angiopoietin-2 blocking antibodies reduce early

101

atherosclerotic plaque development in mice

Chapter 6 Histamine and VEGF mediate pro-angiogenic gene expression

and endothelial cell junction formation through common

cellular regulators

Chapter $7 \quad$ General discussion

Chapter 8

Summary

175

Samenvatting

Chapter $9 \quad$ Valorization

Appendices List of abbreviations

189

Acknowledgements

195

Curriculum Vitae

201 



\section{Chapter 1}

General introduction and outline of the thesis

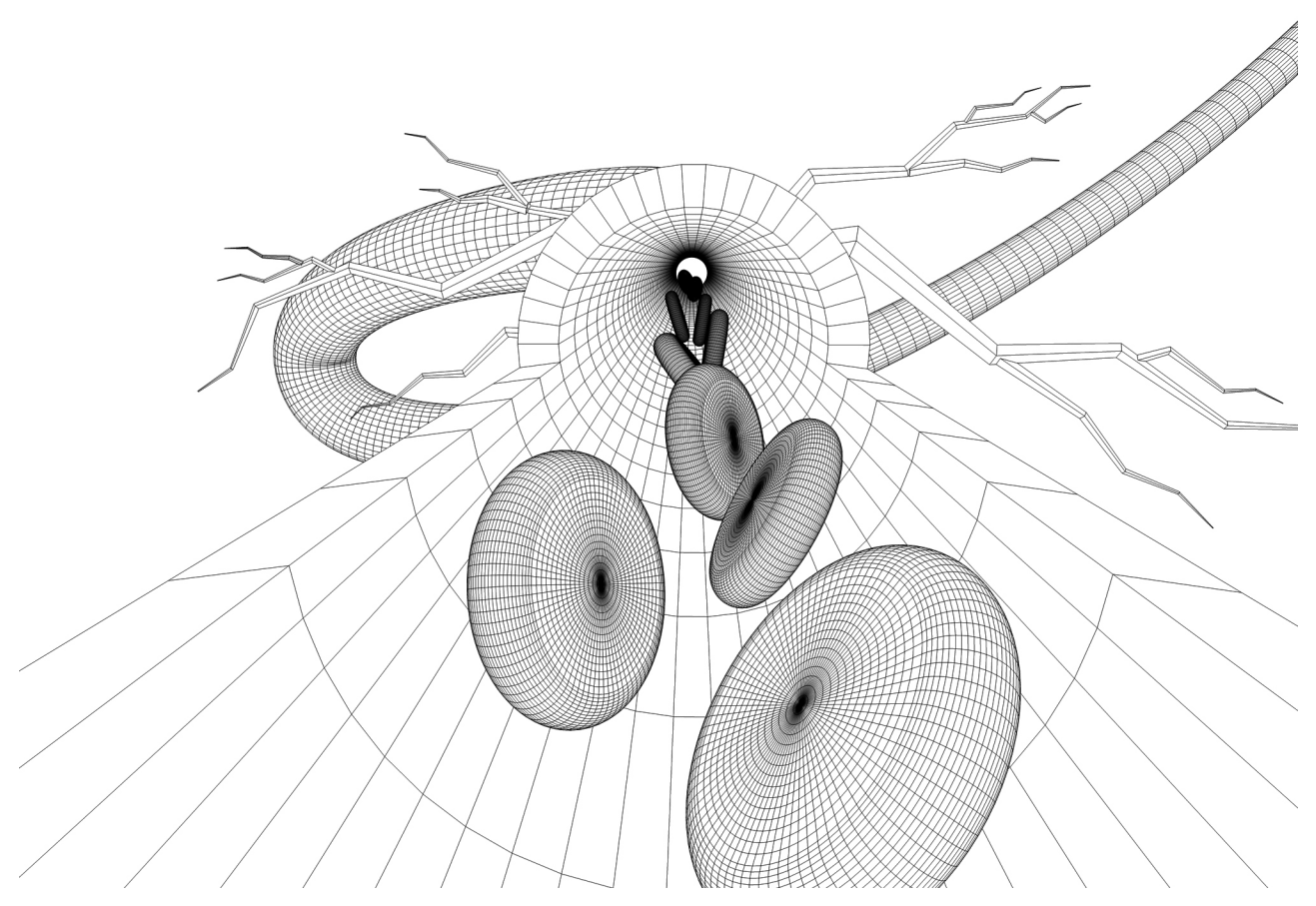




\section{Atherosclerosis and society}

Atherosclerosis is today's leading cause of death worldwide (1), but it is not a recent phenomenon. Tomographic images of around 4000 year old mummies revealed the presence of calcified atherosclerotic plaques in their carotid arteries (2). Atherosclerosis is not only visible with modern technology, it was already recognized around the year 1500 by the famous universal genius and anatomist Leonardo da Vinci during an autopsy of an older man (3). Although atherosclerosis has been studied by pathologists and researchers ever since, the exact disease mechanism still remains unknown.

Risk factors for atherosclerosis include male gender, obesity, smoking, high blood pressure, high plasma levels of low density lipoprotein (LDL) cholesterol and age (4). But it was strikingly demonstrated by Enos et al. in 1953 that atherosclerosis starts early in life. They found in autopsies performed during the Korean war that signs of atherosclerosis were present in over $77 \%$ of the soldiers already in the early twenties (5). This raises two important questions: Why does almost everybody have the disease? And why does not everybody develop clinical symptoms during the course of life?

\section{Atherosclerosis development}

To answer both questions, it is important to understand how an atherosclerotic plaque develops. Atherosclerosis is referred to as a lipid-driven inflammatory disease, but disease initiation is thought to start with the endothelial cells; the inner lining of a blood vessel. Endothelial cells become activated by shear stress. Shear stress is caused by turbulences in blood flow, typically near branch points of the arterial system (6). It is thought that activation of endothelial cells causes changes in cell morphology, triggers apoptosis and endothelial dysfunction, via reduction in nitric oxide and induction of endothelin-1, in this way allowing circulating LDL cholesterol to enter the subendothelial space (7). LDL cholesterol becomes oxidized (oxLDL) enzymatically by for instance myeloperoxidase, or lipoxygenases, or by naturally occurring reactive oxygen species. The retained oxLDL triggers an inflammatory signaling cascade in endothelial cells. They express chemokines, cytokines and adhesion molecules that cause the attraction and activation of inflammatory cells from the circulation $(8,9)$.

Predominantly monocytes are recruited by the endothelial cells in order to initiate the removal of the deposited oxLDL. To this end the extravasated monocytes differentiate into macrophages which engulf the free oxLDL. Macrophages finally turn into foam cells, named after the microscopic appearance as lipid laden foamy cell due to the excessive lipid endocytosis. (10) This lipid overload finally leads to programmed cell death (apoptosis) of foam cells. Apoptotic cells are normally immediately cleared by surrounding macrophages (efferocytosis). However, the efferocytosis system is defective in atherosclerosis and thus apoptotic foam cells accumulate. The exact mechanism for defective efferocytosis are unknown, but amongst others reduced activity or cleavage of the efferocytosis receptor MerTK is a likely explanation (11). The accumulation of apoptotic and/or necrotic cells evokes an even stronger inflammatory response (12). The potentiated immune response leads to an attraction of more immune cells and initiates a vicious circle of cell death and immune cell infiltration. The consequence of this vicious circle is the formation of a necrotic core consisting of cell debris and free cholesterol within the arterial plaque. 
In parallel, smooth muscle cells migrate from the media (medial layer of a blood vessels consisting mainly of smooth muscle cells) into the intima. Smooth muscle cells initiate the formation of a fibrous cap by synthetizing extracellular matrix components such as collagen. The newly formed fibrous cap shields the plaque from the blood stream and is thought to prevent the plaque from rupturing. (8) However, the fibrous cap becomes thinner during disease progression as macrophages infiltrate the cap region and synthetize matrix degrading enzymes, matrix-metallo-proteases (MMPs). MMPs are able to cleave for instance collagen type I, the most common plaque collagen, thereby thinning the fibrous cap and increasing rupture susceptibility (13).

\section{Plaque rupture}

The rupture of an atherosclerotic plaque may have life threatening consequences for the patient, such as stroke or myocardial infarction. Once the content of a plaque is in contact with the blood stream an immediate thrombotic response is initiated by circulating platelets (14). The resulting thrombus may completely occlude the blood vessel at the location of rupture (heart) or dissociates and relocates to smaller blood vessels (brain). The imminent occlusion of smaller blood vessels rapidly causes oxygen-shortage (ischemia) and may finally result in loss of tissue functionality, fibrosis and even necrosis.

The exact underlying mechanisms causing plaque rupture are unknown but several major observations could be linked with plaques at risk for rupture, mostly through imaging and autopsy studies: perivascular-Inflammation, an enlarged necrotic core, a thin fibrous cap, micro-calcification, intra-plaque hemorrhage, plaque neo-angiogenesis and hypoxia $(8,15)$. Besides human imaging and autopsy studies, especially animal models for atherosclerosis have been used to characterize these risk factors. Frequently used animal models for atherosclerosis research are New Zealand white rabbits, pigs, and since the discovery in the 1990s apolipoprotein E (ApoE) and LDL receptor (LDLr) knockout mice (16).

Perivascular inflammatory cells such as macrophages, T cells, B cells and dendritic cells are already present in the adventitia of non-diseased murine arteries and proliferate during disease progression. It has been shown that this immune cell proliferation even leads to the formation of plaque-associated adventitial tertiary lymphoid organs in ApoE KO mice $(17,18)$. In humans perivascular inflammation in the adventitia and media correlated with disease severity and cardiac death in pathological specimens from patients with unstable angina (19).

A large necrotic core has been associated with an increased risk of plaque rupture in human autopsy studies (20). Mechanistically, the presence of a large necrotic core increases the circumferential tensile stress on the fibrous cap. This accelerated biomechanical force might cause rupture of the thin fibrous cap (21).

The necrotic core, apoptotic cells and extracellular matrix might serve as starting point for the development of calcifications. The mechanism of how these calcifications develop is not understood, but vascular imaging by ultrasound and optical coherence tomography revealed a correlation between spotty calcification and plaques at risk of rupture $(22,23)$. It is thought that smooth muscle cells might transdifferentiate into osteogenic smooth muscle cells which promote vascular calcification (24).

Intra-plaque hemorrhage, or the leakage of blood components (erythrocytes, leukocytes, platelets and plasma) into plaques, has been associated with more advanced stages of the 
disease and in particular with rupture prone lesions (25). The hemorrhage originates from either intimal intra-plaque microvessels, or healed thrombi of previous plaque ruptures (26). Erythrocytes in the plaque lead to cholesterol crystal formation, as their membrane contains up to $40 \%$ cholesterol, more than any other celltype. This in turn leads to an expansion of the necrotic core. Moreover, hemorrhages are also thought to increase reactive oxygen species and lipid oxidation via the release of Fe++ from hemoglobin during erythrocyte lysis and myeloperoxidase expressed by neutrophils. The increase of cholesterol oxidation enables an increased recognition of cholesterol by macrophages, and thus promotes foam cell formation. In addition to myeloperoxidase, neutrophils are also a source of proteolytic enzymes that are thought to reduce plaque stability via the degradation of extracellular matrix components $(25,27)$.

Besides the aforementioned risk factors for plaque rupture, also plaque hypoxia and plaque angiogenesis have been associated with rupture prone atherosclerosis. The role of both plaque hypoxia and plaque angiogenesis as risk factors is elucidated in greater detail in the following sections.

\section{Plaque rupture and plaque hypoxia}

Oxygen shortage (hypoxia) within plaques of major and midsized arteries seems paradoxical as the purpose of these vessels is the distribution of freshly oxygenized blood throughout the body. Several studies have shown that exposure of hypercholesterolemic animals (mostly rabbits) to hypoxia leads to an increase in arterial cholesterol and thus an increase in atherosclerosis (28-30). The methods to induce hypoxia in animals are fairly creative, and ranged from breathing of nitrogen (31), housing at constant $10 \%$ oxygen atmosphere (32), housing at simulated high altitude (33), to physical relocation of cholesterol fed rabbits to a mountain top (control group 4,000ft and experimental group 12,470ft above sea level (34)). Although hypoxia or induced hypoxia have been clearly linked to increased atherosclerosis, Greiner (35) made an interesting and logical statement: not many humans are constantly exposed to hypoxia or high altitudes, but still a lot of people suffer from atherosclerosis. This statement implicates that the source of hypoxia, if the hypothesis holds, has to be rather internal than external. Two major sources of internal hypoxia could be identified, cigarette smoke and sleep apnea. The first is assumed to cause hypoxia via increased carbon monoxide although enhanced plaque hypoxia was not studied in this model of cigarette smoking baboons (36). Sleep apnea represents chronic intermittent hypoxia (37), which is also correlated with an increased risk of cardiovascular events. These cases still do not account for all people suffering from atherosclerosis as not everybody undergoing a myocardial infarction or stroke, uses cigarettes or suffer from sleep apnea. Besides these external causes for oxygen shortage, there are two conceivable internal mechanisms of plaque hypoxia in the wall of a main artery: I) large plaque size and II) the metabolic activity of the plaque.

Atheroma can reach a size that is sufficient to limit the residual arterial lumen leading to distal ischemia, but more importantly exceeds the oxygen tissue diffusion distance of 100 $250 \mu \mathrm{m}$ (38). Although the oxygen diffusion distance is a reasonable explanation for hypoxia in human atherosclerosis, also murine plaques were positive for hypoxia-specific markers $(39,40)$. Thus, besides the oxygen diffusion distance, there should be another explanation. It has been shown in human atherosclerosis that areas in plaque with high grade 
inflammation are positive for tracers of metabolic activity, such as fluorine-labeled 2-deoxyD-glucose (FDG, radioactively labeled glucose analogue) indicating a high oxygen consumption rate $(41,42)$. Together with experimental data on the high glucose demand of hypoxic macrophages, this indicates metabolic activity, rather than oxygen diffusion distance, as a main source of plaque hypoxia (43).

As these observational data do not provide any insight in the mechanism of plaque hypoxia, it is important to understand the physiological reaction to hypoxia. Molecularly, the cellular hypoxia response (Figure 1) is regulated via the prolyl hydroxylase domain proteins (PHD). Besides iron and 2-oxaloglutarate, oxygen is the major cofactor of the PHD enzyme. The binding of oxygen enables the PHD enzyme to hydroxylate the transcription factor hypoxia inducible factor 1 alpha (HIF1 $\alpha$ ). Upon hydroxylation HIF1 $\alpha$ is ubiquitinylated by the von Hippel-Lindau enzyme ( $\mathrm{pVHL}$ ) and subsequently degraded in the $26 \mathrm{~S}$ proteasome complex. Conversely, in the absence of oxygen the PHD enzyme is inhibited due to its missing cofactor oxygen and HIF1 $\alpha$ remains stable and translocates to the nucleus. In the nucleus, HIF1 $\alpha$ binds to its constitutively expressed subunit HIF1 $\beta$ and upon dimerization to a distinct DNA promoter region, the hypoxia responsible element (HRE). HIF-HRE binding facilitates the transcription of hypoxia related genes for instance for the cellular oxygen metabolism (glucose transporter (GLUT1) etc.), and for angiogenesis (vascular endothelial growth factor (VEGF)). The goal of the molecular hypoxia response is decreasing cellular oxygen consumption/demand and restoring oxygen supply via the cellular stimulation of angiogenesis (44). Besides HIF1 $\alpha$ also its isoform HIF2 $\alpha$ is regulated by the PHD enzyme, however, HIF2 $\alpha$ is not relevant for the work presented in this thesis.
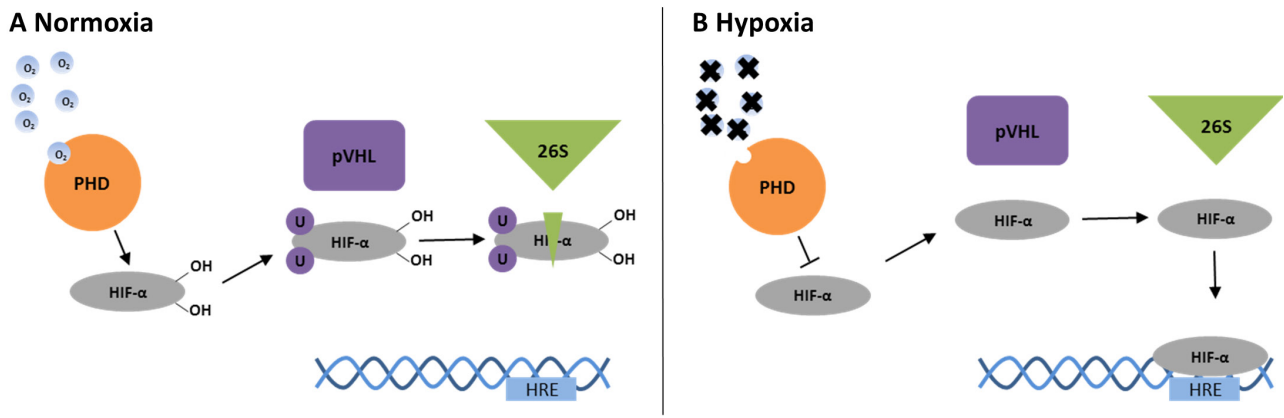

Figure 1. Schematic overview regulation of molecular hypoxia response by PHD

(A) Under normoxia PHD binds to its co-factor oxygen and hydroxylates (OH) HIF1 $\alpha$. The hydroxylation enables ubiquitinylation $(\mathrm{U})$ by the $\mathrm{pVHL}$ and subsequent degradation by the $26 \mathrm{~S}$ proteasome. (B) Under hypoxia HIF1 $\alpha$ remains stable and is able to initiate a transcriptomic response of hypoxia related genes via the binding to the $\mathrm{HRE}$.

Human plaque specimens were positive for the hypoxia specific marker pimonidazole (45), and increased levels of HIF1 $\alpha$ were found in several studies (46-48). HIF1 $\alpha$ regulates processes that are negatively associated with disease outcome in general. Elevated HIF1 $\alpha$ levels could be correlated with increased expression of the angiogenic mediator VEGF and increased plaque angiogenesis (45). On a cellular level, increased HIF1 $\alpha$ induces a shift in the energy metabolism towards anaerobic glycolysis, predominantly in macrophages. This in turn skews macrophages to a -inflammatory phenotype (49). To understand the contribution of HIF1 $\alpha$ responses in relevant cell types to atherosclerosis several transgenic mice were generated (Table 1): HIF1 $\alpha$ KO in macrophages had no effect (50); HIF1 $\alpha$ KO in 
endothelial cells led to reduction in plaque size via decreased monocyte recruitment (51); HIF1 $\alpha$ KO in dendritic cells increased plaque size via increased T cell infiltration (50), local adenoviral HIF1 $\alpha$ inhibition resulted in decreased whereas local overexpression increased plaque size (52) and systemic HIF1 overexpression resulted in decreased plaque size via the downregulation of interferon $\gamma$ (53). These experimental data allow no clear conclusion about the beneficial or adverse effects of HIF1 $\alpha$ in atherosclerosis, as effects seem celltype dependent.

A simpler manner to reverse the observed detrimental effects of plaque hypoxia is the use of hyperoxic gas. Already in 1954, Altschul et al. treated hypercholesterolemic rabbits with hyperoxic gas and could show a reduction in plaque size (54). The results were confirmed in 1969 by Kjeldsen et al. who also reported a reduction in plaque size after inhalation of hyperoxic gas in rabbits (55). These findings prove the effectivity of this simple approach, though molecular insights in the underlying molecular mechanism were absent and direct effects on in plaque hypoxia were not analyzed. The translation of this treatment into clinical practice, however, is not feasible and thus a pharmacological approach seems more logical.

In addition to HIF1 $\alpha$, also its master regulators, the PHD enzymes, may represent suitable drug targets. It should be noted that besides HIF1 $\alpha$, PHDs have several other binding partners (56), three of which have an established link with atherosclerosis: activation transcription factor 4 (ATF4), $\beta(2)$-adrenergic receptor ( $\beta(2) A R$ ) and inhibitor of kappa $B$ kinase $\beta$ (IKK $\beta$ ). These targets are regulated in an isoform specific manner: the activity of ATF4 is regulated by PHD1 and $3(57,58), \beta(2)$ AR degradation is regulated by PHD3 (59) and the canonical pathway of nuclear factor kappa beta (NFKB) is negatively regulated by all three PHD isoforms (60-62). Thus, increasing PHD2 may not only lower HIF1 $\alpha$ levels, but also decrease the pro-inflammatory canonical NFkB pathway. On the contrary, PHD2 deletion has been proven beneficial as it protects from diabetes and obesity (63).

To conclude, hypoxia is present in atherosclerotic plaques and is negatively associated with disease outcome and progression. In line, reversal by hyperoxic gas is beneficial, but inference with the cellular hypoxia pathway yields inconclusive results and was never attributed to direct changes in plaque hypoxia. Further investigation is needed to unravel the exact underlying mechanism of plaque hypoxia und to translate the findings into a therapeutic intervention.

\section{Plaque rupture and plaque microvessels}

In addition to hypoxia, also plaque microvessels have been associated with negative disease outcome in atherosclerosis. Under physiological circumstances, microvessels originate from the adventitia and provide the muscular media with oxygen and nutrients (64). It is thought that the physiological role of microvessels in atherosclerosis is drainage of lipids, nutrient supply and re-oxygenation (65). Therefore, it is postulated that plaque angiogenesis is likely initiated by plaque hypoxia. Hypoxia and the related HIF1 $\alpha$ expression are a wellestablished trigger for angiogenesis and both have been identified in atherosclerotic plaques (45). Furthermore, hypoxia/HIF1 $\alpha$-induced angiogenic growth factors have been found in human atherosclerosis, such as VEGF, angiopoietin (Ang) 1 and 2 (66-69). In addition to hypoxia, also inflammatory cells are thought to contribute to angiogenesis induction (70). 
The presence of microvessels has been linked to plaque rupture and cardiovascular events in human autopsy studies $(71,72)$. Several experimental studies support this link, as induced angiogenesis increased plaque burden in all studies. Here, different methods of inducing angiogenesis in combination with different animal models (mouse, rabbit and pig) were used. Plaque angiogenesis was induced by stress $(73,74)$, treatment with pro-inflammatory mediators $(75)$, pro-angiogenic growth factors $(67,76)$ and viral gene delivery of proangiogenic factors (77-83). However, not only an increased number of microvessels was shown to have adverse effects on disease outcome, but also microvessel quality has emerged as a risk factor for human plaque rupture. Microvessel quality is determined by its ultrastructural composition: the morphology and integrity of endothelial cells, their junctions, and the extent of pericyte coverage. Microvessels from pathological specimens of ruptured plaques in coronary arteries displayed detachments of the endothelial junctions, endothelial membrane blebs and a thin or absent endothelial basement membrane. In addition to the endothelial malformation, surrounding pericytes were found to be absent in a majority of microvessels in ruptured plaques.(84) These ultrastructural characteristics suggest a leaky microvessel phenotype known from tumor vessels (85). Vascular leakage might result in increased extravasation of immune cells and deposition of lipids and red blood cells in the plaques $(72,86)$. Therefore, these microvessels are thought to represent one of the main sources of intra-plaque hemorrhage, in addition to healed thrombi (27).

Microvessel leakage might have three underlying causes in atherosclerosis: I) defective angiogenesis II) defective vascular maintenance/quiescence III) acute vascular permeability. The following paragraphs will highlight processes in physiological angiogenesis, sensitive to changes that may induce microvascular hyper-permeability.

Physiological angiogenesis is a complex molecular process that relies on the interplay between different cell types and the optimal balance of pro- and anti-angiogenic factors (Figure 2). Upon stimulation of an existing vessel with proangiogenic factors, such as VEGF, fibroblast growth factors (FGFs) and Ang-2, pericytes detach from the endothelial cells. Subsequently, endothelial cell junctions loosen and proteases are released to prepare the surrounding extracellular matrix (ECM) for angiogenesis and free ECM bound angiogenic molecules. Integrin signaling stimulates the movement of endothelial cells into the ECM space. Vessel sprouting is led by one endothelial cell (tip cell) along a gradient of angiogenic factors. The tip cell is followed by dividing endothelial cells (stalk cells) assuring vessel outgrowth. A lumen is formed via the expression of amongst others vascular endothelial (VE) cadherin and VEGF by the endothelial cells. Finally, pericyte coverage is induced by the secretion of Ang-1, platelet derived growth factor B (PDGF-B) and tumor growth factor- $\beta$ (TGF- $\beta$ ); and a basement membrane is established (87). If these processes are disturbed, leakage might be induced, as illustrated by severe blood-brain permeability after prevention of matrix-retention of PDGFB by a retention motif KO. Another example of a disturbance in growth factor balance has been shown for VEGF as increased levels have been found in leaky tumor vessels (88). Similarly immature leaky microvessels in atherosclerosis also correlate with high levels of VEGF (89). 


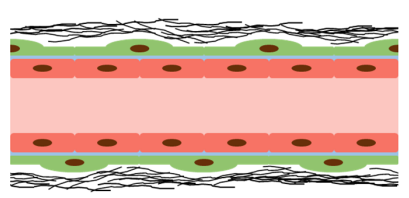

A Quiescent state

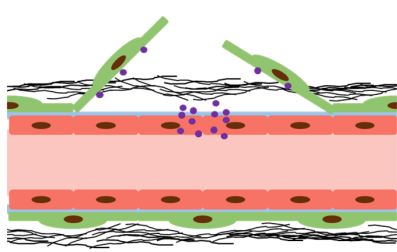

B Angiogenic stimulus and pericyte detachment

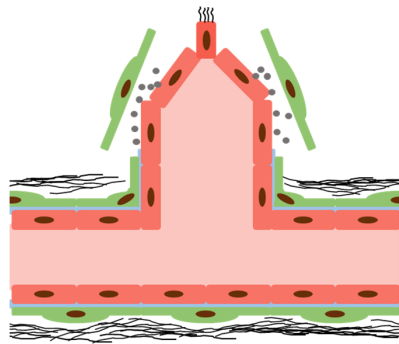

E Lumen formation and pericyte recruitment

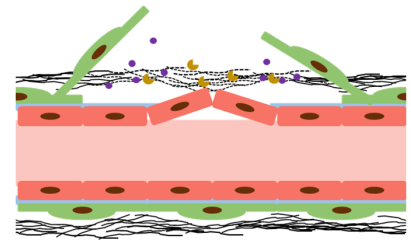

C ECM degradation and EC detachment

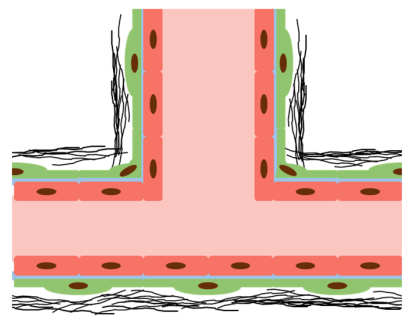

F Quiescent state

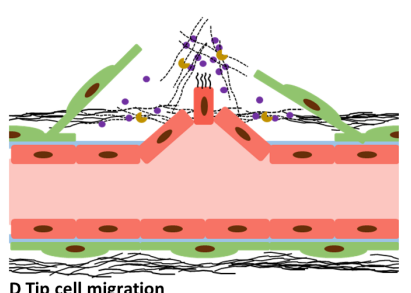

D Tip cell migration

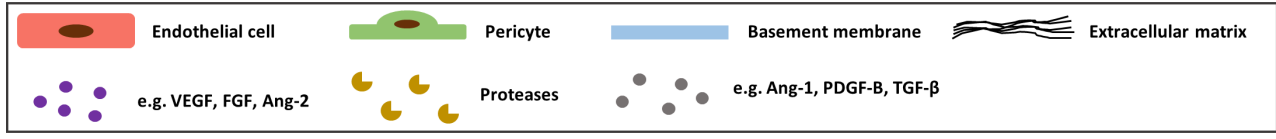

Figure 2. Schematic overview angiogenesis

(A) Vessel in its quiescent state. (B) Angiogenic stimulus by e.g. VEFG, FGF, Ang-2 causes first pericyte detachment and (C) second endothelial cell (EC) detachment and facilitated by protease secretion migration into the extracellular matrix. (D) Neo-vessel outgrowth along growth factor gradient guided by tip cell. (E) Lumen establishment, pericyte recruitment and basement membrane formation stimulated by e.g. Ang-1, PDGF-B, TGF$\beta$. (F) Newly formed quiescent vascular branch.

Once a vessel has been formed, its quiescent state has to be maintained in order to function physiologically. Here, autocrine Ang-1 has a prominent role, as binding to its receptor Tie2 inhibits VEGF induced permeability. Furthermore, it was shown that Tie2 receptors of neighboring endothelial cells dimerize at the junction upon Ang-1 binding, thereby contributing to junction stability. In addition, Ang-1 signaling is able to restore pericyte coverage. The beneficial effects of Ang-1 are counteracted by its natural antagonist Ang-2 (90). High Ang-2 levels have been associated with increased plaque angiogenesis in culprit lesions and potentially also with microvessel instability suggesting increased leakage (68).

Ang-2 also has a potential role in acute vascular permeability induced by inflammatory mediators such as histamine, bradykinin and VEGF. These mediators are able to induce rapid and robust hyper-permeability but only in the presence of Ang-2 (91). The same might be true for atherosclerosis as plaques with high numbers of histamine-producing mast cells are associated with increased intra-plaque hemorrhage and cardiovascular events (92). The direct effect of the proangiogenic factors on microvessel permeability in atherosclerosis is difficult to distinguish from their prominent role in angiogenesis. However, the potential to evoke hyper-permeability has been extensively investigated in other disease models, which strongly suggests a similar role in atherosclerosis.

Overall, two therapeutic strategies arise from the current knowledge about angiogenesis in atherosclerosis: I) angiogenesis inhibition might be beneficial when assuming that high numbers of microvessels are associated with cardiovascular events and II) restoration of 
vascular functionality and/or quiescence might prevent leakage of pre-existing microvessels and subsequent cardiovascular events.

Inhibition of angiogenesis in atherosclerosis has been studied in various experimental animal models using different approaches such as pharmacological angiogenesis inhibitors (93-100), vascular growth factor antibodies $(101,102)$ and vaccination for vascular growth factor receptors (103-105). All studies conclude that inhibition of angiogenesis decreased atherosclerosis and increases plaque stability (93-105). However, several of these studies hardly find any intra-plaque microvessels at baseline and the treatment was often started together with atherosclerosis induction. This makes a translation to humans difficult as microvessels are already present at time of clinical complications. Thus restoring microvessel functionality might be a more relevant clinical approach (106). 


$$
\text { | }
$$




\section{Overall aim and hypothesis}

The overall aim of this thesis is to explore a possible causal role for plaque hypoxia and angiogenesis in experimental murine atherosclerosis, as both have been identified as risk factors for plaque rupture in humans.

We hypothesize that:

I. Hyperoxic treatment alleviates plaque hypoxia and atherosclerosis burden and the underlying molecular mechanism can be identified (chapter 2).

II. Aggravation of the cellular hypoxia response via myeloid PHD2 deficiency increases atherosclerosis burden via elevated hypoxia signaling and increased plaque angiogenesis (chapter $\mathbf{3}$ ).

III. Microvascular pericyte loss via PDGF-B retention motif $\mathrm{KO}$ and subsequent increased microvascular permeability exacerbates atherosclerosis (chapter 4).

IV. Microvessel normalization via anti-Ang-2 treatment reduces atherosclerosis progression (chapter 5 ).

V. Comparison of histamine, and VEGF-A, D and F induced angiogenic response and microvascular permeability unravels overlapping and independent stimulation of relevant pathways in the endothelial transcriptome (chapter 6).

\section{Outline of the thesis}

Plaque hypoxia is associated with an adverse disease outcome and hyperoxic treatment seems beneficial (54). However, hyperoxic treatment is rather impractical on a large scale and therefore the molecular mechanism by which hyperoxic therapy ameliorates atherosclerosis was investigated (chapter 2) by treating pre-existing plaques in atherosclerosis-prone LDLr KO mice with hyperoxic carbogen gas $\left(95 \% \mathrm{O}_{2}\right)$.

As hypoxia is a known stimulus of angiogenesis it is conceivable that it also triggers angiogenesis in atherosclerosis. In chapter $\mathbf{3}$ cellular hypoxia was enhanced by a myeloid $\mathrm{KO}$ of the hypoxia master regulator PHD2 and intra-plaque and adventitial microvessels and atherosclerotic plaque characteristics were studied in LDLr KO mice.

As not only microvessel quantity, but also microvessel quality, has been associated with consequences on human atherosclerosis (84), structurally defective microvessels in murine atherosclerosis were modeled in chapter 4 . Therefore, microvessel pericyte loss was instigated in atherosclerosis-prone mice by breeding PDGF-B retention motif KO (107) LDLr KO mice.

In contrast to the generation of defective microvessels in chapter 4, microvessels in preexisting atherosclerosis were normalized in chapter 5. LDLr receptor KO mice with preexisting plaques were treated with an antibody for the vascular growth factor Ang-2 and effects on atherosclerosis were analyzed.

Defective microvessels are a major source of intra-plaque hemorrhage in humans and intraplaque hemorrhage is thought to originate from microvessel hyper-permeability. Hyperpermeability, in addition to angiogenesis, is induced by members of the VEGF family, but also by histamine. The potential of VEGF to induce vascular permeability and angiogenesis in comparison to histamine is unknown. In chapter 6 the elicited transcriptomic response related to permeability induction and angiogenic capacity of these compounds was therefore compared. Their stimulation of the transcriptomic response was studied in 
Chapter 1

primary human umbilical cord endothelial cell using RNA sequencing. In addition, the permeability induction capacity was studied after injection of the compounds in wild type mice. 


\section{References}

1. WHO. Fact Sheet Cardiovascular Diseases [Internet]. Fact Sheet No 317. 2015 [cited 2015 Oct 27]. Available from: http://www.who.int/mediacentre/factsheets/fs317/en/

2. Thompson RC, Allam AH, Lombardi GP, Wann LS, Sutherland ML, Sutherland JD, et al. Atherosclerosis across 4000 years of human history: the Horus study of four ancient populations. Lancet (London, England). Elsevier Ltd; $2013 \mathrm{Apr}$ 6;381(9873):1211-22.

3. Boon B. Leonardo da Vinci on atherosclerosis and the function of the sinuses of Valsalva. Neth Heart J. 2009 Dec;17(12):496-9.

4. Fruchart J-C, Nierman MC, Stroes ESG, Kastelein JJP, Duriez P. New risk factors for atherosclerosis and patient risk assessment. Circulation. 2004 Jun 15;109(23 Suppl 1):III15-9.

5. Enos WF, Holmes RH, Beyer J. Coronary disease among United States soldiers killed in action in Korea; preliminary report. J Am Med Assoc. 1953 Jul 18;152(12):1090-3.

6. Cheng C, Tempel D, van Haperen R, van der Baan A, Grosveld F, Daemen MJAP, et al. Atherosclerotic lesion size and vulnerability are determined by patterns of fluid shear stress. Circulation. 2006 Jun $13 ; 113(23): 2744-53$.

7. Wentzel JJ, Chatzizisis YS, Gijsen FJH, Giannoglou GD, Feldman CL, Stone PH. Endothelial shear stress in the evolution of coronary atherosclerotic plaque and vascular remodelling: current understanding and remaining questions. Cardiovasc Res. 2012 Nov 1;96(2):234-43.

8. Bentzon JF, Otsuka F, Virmani R, Falk E. Mechanisms of plaque formation and rupture. Circ Res. 2014 Jun 6;114(12):1852-66.

9. Weber C, Noels H. Atherosclerosis: current pathogenesis and therapeutic options. Nat Med. Nature Publishing Group; 2011 Nov 7;17(11):1410-22.

10. Hansson GK. Inflammation, atherosclerosis, and coronary artery disease. N Engl J Med. 2005 Apr 21;352(16):1685-95.

11. Moore KJ, Tabas I. Macrophages in the pathogenesis of atherosclerosis. Cell. Elsevier; 2011 Apr 29;145(3):341-55.

12. Tabas I. Macrophage death and defective inflammation resolution in atherosclerosis. Nat Rev Immunol. 2010 Jan;10(1):36-46.

13. Libby P. Mechanisms of acute coronary syndromes and their implications for therapy. N Engl J Med. 2013 May 23;368(21):2004-13.

14. Fernández-Ortiz A, Badimon JJ, Falk E, Fuster V, Meyer B, Mailhac A, et al. Characterization of the relative thrombogenicity of atherosclerotic plaque components: implications for consequences of plaque rupture. J Am Coll Cardiol. 1994 Jun;23(7):1562-9.

15. Stone GW, Maehara A, Lansky AJ, de Bruyne B, Cristea E, Mintz GS, et al. A prospective natural-history study of coronary atherosclerosis. N Engl J Med. 2011 Jan 20;364(3):226-35.

16. Shim J, Al-Mashhadi RH, Sørensen CB, Bentzon JF. Large animal models of atherosclerosis - new tools for persistent problems in cardiovascular medicine. J Pathol. 2015 Sep 28;914531200.

17. Moos MPW, John N, Gräbner R, Nossmann S, Günther B, Vollandt R, et al. The lamina adventitia is the major site of immune cell accumulation in standard chow-fed apolipoprotein E-deficient mice. Arterioscler Thromb Vasc Biol. 2005 Nov;25(11):2386-91.

18. Galkina E, Kadl A, Sanders J, Varughese D, Sarembock IJ, Ley K. Lymphocyte recruitment into the aortic wall before and during development of atherosclerosis is partially L-selectin dependent. J Exp Med. 2006 May 15;203(5):1273-82. 
19. Kohchi K, Takebayashi S, Hiroki T, Nobuyoshi M. Significance of adventitial inflammation of the coronary artery in patients with unstable angina: results at autopsy. Circulation. 1985 Apr;71(4):709-16.

20. Virmani R, Burke AP, Farb A, Kolodgie FD. Pathology of the vulnerable plaque. J Am Coll Cardiol. 2006 Apr 18;47(8 Suppl):C13-8.

21. Gertz SD, Roberts WC. Hemodynamic shear force in rupture of coronary arterial atherosclerotic plaques. Am J Cardiol. 1990 Dec 1;66(19):1368-72.

22. Kataoka Y, Puri R, Hammadah M, Duggal B, Uno K, Kapadia SR, et al. Spotty calcification and plaque vulnerability in vivo: frequency-domain optical coherence tomography analysis. Cardiovasc Diagn Ther. 2014 Dec;4(6):460-9.

23. Ehara S, Kobayashi Y, Yoshiyama M, Shimada K, Shimada Y, Fukuda D, et al. Spotty calcification typifies the culprit plaque in patients with acute myocardial infarction: an intravascular ultrasound study. Circulation. 2004 Nov 30;110(22):3424-9.

24. Willems B a. G, Vermeer C, Reutelingsperger CPM, Schurgers LJ. The realm of vitamin K dependent proteins: shifting from coagulation toward calcification. Mol Nutr Food Res. 2014 Aug;58(8):1620-35.

25. Kolodgie FD, Gold HK, Burke AP, Fowler DR, Kruth HS, Weber DK, et al. Intraplaque hemorrhage and progression of coronary atheroma. N Engl J Med. 2003 Dec 11;349(24):2316-25.

26. Michel J-B, Martin-Ventura JL, Nicoletti A, Ho-Tin-Noé B. Pathology of human plaque vulnerability: mechanisms and consequences of intraplaque haemorrhages. Atherosclerosis. Elsevier Ltd; 2014 Jun;234(2):311-9.

27. Michel J-B, Virmani R, Arbustini E, Pasterkamp G. Intraplaque haemorrhages as the trigger of plaque vulnerability. Eur Heart J. 2011 Aug;32(16):1977-85, 1985a, 1985b, 1985c.

28. Nakano D, Hayashi T, Tazawa N, Yamashita C, Inamoto S, Okuda N, et al. Chronic hypoxia accelerates the progression of atherosclerosis in apolipoprotein E-knockout mice. Hypertens Res. 2005 Oct;28(10):83745.

29. Garbarsch C, Matthiessen ME, Helin P, Lorenzen I. Arteriosclerosis and hypoxia. J Atheroscler Res. 1969 May;9(3):283-94.

30. Kjeldsen K, Astrup P, Wanstrup J. Reversal of rabbit atheromatosis by hyperoxia. J Atheroscler Res. 1969 Sep;10(2):173-8.

31. Helin G, Helin P, Lorenzen I. The aortic glycosaminoglycans in arteriosclerosis induced by systemic hypoxia. Atherosclerosis. 1970 Sep;12(2):235-40.

32. Lindy S, Turto H, Uitto J, Garbarsch C, Helin P, Lorenzen I. The effect of chronic hypoxia on lactate dehydrogenase in rabbit arterial wall Biochemical studies on normal and injured aortas. Atherosclerosis. 1974 Sep;20(2):295-301.

33. Frith $\mathrm{CH}, \mathrm{McMurtry}$ IF, Alexander AF, Will DH. Influence of hypertension and hypoxemia on arterial biochemistry and morphology in swine. Atherosclerosis. 1974 Sep;20(2):189-206.

34. Zemplenyi T, Tidwell DF, Fronek K. Aortic enzymes and lactate in high altitude-raised and cholesterol-fed rabbits. Atherosclerosis. 1984 Aug;52(2):233-42.

35. Gainer JL, Chisolm GM. Oxygen diffusion and atherosclerosis. Atherosclerosis. 1974 Jan;19(1):135-8.

36. Rogers WR, Bass RL, Johnson DE, Kruski AW, McMahan CA, Montiel MM, et al. Atherosclerosis-related responses to cigarette smoking in the baboon. Circulation. 1980 Jun 18;61(6):1188-93.

37. Marin JM, Carrizo SJ, Vicente E, Agusti AG. Long-term cardiovascular outcomes in men with obstructive sleep apnoea-hypopnoea with or without treatment with continuous positive airway pressure: an observational study. Lancet. 2005 Mar;365(9464):1046-53. 
38. Torres Filho IP, Leunig M, Yuan F, Intaglietta M, Jain RK. Noninvasive measurement of microvascular and interstitial oxygen profiles in a human tumor in SCID mice. Proc Natl Acad Sci U S A. 1994 Mar $15 ; 91(6): 2081-5$.

39. Parathath S, Mick SL, Feig JE, Joaquin V, Grauer L, Habiel DM, et al. Hypoxia is present in murine atherosclerotic plaques and has multiple adverse effects on macrophage lipid metabolism. Circ Res. 2011 Oct 28;109(10):1141-52.

40. Marsch E, Theelen TL, Demandt JAF, Jeurissen M, van Gink M, Verjans R, et al. Reversal of hypoxia in murine atherosclerosis prevents necrotic core expansion by enhancing efferocytosis. Arterioscler Thromb Vasc Biol. 2014 Dec;34(12):2545-53.

41. Tawakol A, Migrino RQ, Bashian GG, Bedri S, Vermylen D, Cury RC, et al. In vivo 18F-fluorodeoxyglucose positron emission tomography imaging provides a noninvasive measure of carotid plaque inflammation in patients. J Am Coll Cardiol. 2006 Nov 7;48(9):1818-24.

42. Tarkin JM, Joshi FR, Rudd JHF. PET imaging of inflammation in atherosclerosis. Nat Rev Cardiol. Nature Publishing Group; 2014 Aug;11(8):443-57.

43. Folco EJ, Sheikine Y, Rocha VZ, Christen T, Shvartz E, Sukhova GK, et al. Hypoxia but not inflammation augments glucose uptake in human macrophages: Implications for imaging atherosclerosis with 18fluorine-labeled 2-deoxy-D-glucose positron emission tomography. J Am Coll Cardiol. 2011 Aug 2;58(6):603-14.

44. Bishop T, Ratcliffe PJ. HIF hydroxylase pathways in cardiovascular physiology and medicine. Circ Res. 2015 Jun 19;117(1):65-79.

45. Sluimer JC, Gasc J-M, van Wanroij JL, Kisters N, Groeneweg M, Sollewijn Gelpke MD, et al. Hypoxia, hypoxia-inducible transcription factor, and macrophages in human atherosclerotic plaques are correlated with intraplaque angiogenesis. J Am Coll Cardiol. 2008 Apr 1;51(13):1258-65.

46. Higashida T, Kanno H, Nakano M, Funakoshi K, Yamamoto I. Expression of hypoxia-inducible angiogenic proteins (hypoxia-inducible factor-1alpha, vascular endothelial growth factor, and E26 transformationspecific-1) and plaque hemorrhage in human carotid atherosclerosis. J Neurosurg. 2008 Jul;109(1):8391.

47. Vink A, Schoneveld AH, Lamers D, Houben AJS, van der Groep P, van Diest PJ, et al. HIF-1 alpha expression is associated with an atheromatous inflammatory plaque phenotype and upregulated in activated macrophages. Atherosclerosis. 2007 Dec;195(2):e69-75.

48. Luque A. Overexpression of hypoxia/inflammatory markers in atherosclerotic carotid plaques. Front Biosci. 2008; Volume(13):6483.

49. Tawakol A, Singh P, Mojena M, Pimentel-Santillana M, Emami H, MacNabb M, et al. HIF-1 $\alpha$ and PFKFB3 Mediate a Tight Relationship Between Proinflammatory Activation and Anerobic Metabolism in Atherosclerotic Macrophages. Arterioscler Thromb Vasc Biol. 2015 Jun;35(6):1463-71.

50. Chaudhari SM, Sluimer JC, Koch M, Theelen TL, Manthey HD, Busch M, et al. Deficiency of HIF1 $\alpha$ in Antigen-Presenting Cells Aggravates Atherosclerosis and Type 1 T-Helper Cell Responses in Mice. Arterioscler Thromb Vasc Biol. 2015 Nov;35(11):2316-25.

51. Akhtar S, Hartmann P, Karshovska E, Rinderknecht F-A, Subramanian P, Gremse F, et al. Endothelial Hypoxia-Inducible Factor-1 $\alpha$ Promotes Atherosclerosis and Monocyte Recruitment by Upregulating MicroRNA-19a. Hypertension. 2015 Oct 19;HYPERTENSIONAHA.115.05886.

52. Christoph M, Ibrahim K, Hesse K, Augstein A, Schmeisser A, Braun-Dullaeus RC, et al. Local inhibition of hypoxia-inducible factor reduces neointima formation after arterial injury in ApoE-/- mice. Atherosclerosis. Elsevier Ltd; 2014 Apr;233(2):641-7.

53. Ben-Shoshan J, Afek A, Maysel-Auslender S, Barzelay A, Rubinstein A, Keren G, et al. HIF-1alpha 
overexpression and experimental murine atherosclerosis. Arterioscler Thromb Vasc Biol. 2009 May;29(5):665-70.

54. ALTSCHUL R, HERMAN IH. Influence of oxygen inhalation on cholesterol metabolism. Arch Biochem Biophys. 1954 Jul;51(1):308-9.

55. Kjeldsen K, Astrup P, Wanstrup J. Reversal of rabbit atheromatosis by hyperoxia. J Atheroscler Res. 1969;10(2):173-8.

56. Wong BW, Kuchnio A, Bruning U, Carmeliet P. Emerging novel functions of the oxygen-sensing prolyl hydroxylase domain enzymes. Trends Biochem Sci. Elsevier Ltd; 2013 Jan;38(1):3-11.

57. Hiwatashi Y, Kanno K, Takasaki C, Goryo K, Sato T, Torii S, et al. PHD1 interacts with ATF4 and negatively regulates its transcriptional activity without prolyl hydroxylation. Exp Cell Res. 2011 Dec 10;317(20):2789-99.

58. Köditz J, Nesper J, Wottawa M, Stiehl DP, Camenisch G, Franke C, et al. Oxygen-dependent ATF-4 stability is mediated by the PHD3 oxygen sensor. Blood. 2007 Nov 15;110(10):3610-7.

59. Xie L, Xiao K, Whalen EJ, Forrester MT, Freeman RS, Fong G, et al. Oxygen-Regulated 2-Adrenergic Receptor Hydroxylation by EGLN3 and Ubiquitylation by pVHL. Sci Signal. 2009 Jul 7;2(78):ra33-ra33.

60. Klotzsche-von Ameln A, Muschter A, Mamlouk S, Kalucka J, Prade I, Franke K, et al. Inhibition of HIF prolyl hydroxylase-2 blocks tumor growth in mice through the antiproliferative activity of TGF $\beta$. Cancer Res. 2011 May 1;71(9):3306-16.

61. Cummins EP, Berra E, Comerford KM, Ginouves A, Fitzgerald KT, Seeballuck F, et al. Prolyl hydroxylase-1 negatively regulates IkappaB kinase-beta, giving insight into hypoxia-induced NFkappaB activity. Proc Natl Acad Sci U S A. 2006 Nov 28;103(48):18154-9.

62. Fu J, Taubman MB. Prolyl hydroxylase EGLN3 regulates skeletal myoblast differentiation through an NFkappaB-dependent pathway. J Biol Chem. 2010 Mar 19;285(12):8927-35.

63. Rahtu-Korpela L, Karsikas S, Hörkkö S, Blanco Sequeiros R, Lammentausta E, Mäkelä KA, et al. HIF prolyl 4-hydroxylase-2 inhibition improves glucose and lipid metabolism and protects against obesity and metabolic dysfunction. Diabetes. 2014 Oct;63(10):3324-33.

64. Buerk DG, Goldstick TK. Arterial wall oxygen consumption rate varies spatially. Am J Physiol. 1982 Dec;243(6):H948-58.

65. Gössl M, Versari D, Lerman LO, Chade AR, Beighley PE, Erbel R, et al. Low vasa vasorum densities correlate with inflammation and subintimal thickening: potential role in location--determination of atherogenesis. Atherosclerosis. 2009 Oct;206(2):362-8.

66. Shweiki D, Itin A, Soffer D, Keshet E. Vascular endothelial growth factor induced by hypoxia may mediate hypoxia-initiated angiogenesis. Nature. 1992 Oct 29;359(6398):843-5.

67. Celletti FL, Waugh JM, Amabile PG, Brendolan A, Hilfiker PR, Dake MD. Vascular endothelial growth factor enhances atherosclerotic plaque progression. Nat Med. 2001 Apr;7(4):425-9.

68. Post S, Peeters W, Busser E, Lamers D, Sluijter JPG, Goumans M-J, et al. Balance between angiopoietin-1 and angiopoietin-2 is in favor of angiopoietin-2 in atherosclerotic plaques with high microvessel density. J Vasc Res. 2008;45(3):244-50.

69. Calvi C, Dentelli P, Pagano M, Rosso A, Pegoraro M, Giunti S, et al. Angiopoietin 2 Induces Cell Cycle Arrest in Endothelial Cells: A Possible Mechanism Involved in Advanced Plaque Neovascularization. Arterioscler Thromb Vasc Biol. 2004 Mar 1;24(3):511-8.

70. Cheng C, Chrifi I, Pasterkamp G, Duckers HJ. Biological mechanisms of microvessel formation in advanced atherosclerosis: the big five. Trends Cardiovasc Med. Elsevier; 2013 Jul;23(5):153-64. 
71. Hellings WE, Peeters W, Moll FL, Piers SRD, van Setten J, Van der Spek PJ, et al. Composition of carotid atherosclerotic plaque is associated with cardiovascular outcome: a prognostic study. Circulation. 2010 May 4;121(17):1941-50.

72. Virmani R, Kolodgie FD, Burke AP, Finn A V, Gold HK, Tulenko TN, et al. Atherosclerotic plaque progression and vulnerability to rupture: angiogenesis as a source of intraplaque hemorrhage. Arterioscler Thromb Vasc Biol. 2005 Oct;25(10):2054-61.

73. Najafi AH, Aghili N, Tilan JU, Andrews JA, Peng X, Lassance-Soares RM, et al. A new murine model of stress-induced complex atherosclerotic lesions. Dis Model Mech. 2013 Mar;6(2):323-31.

74. Roth L, Rombouts M, Schrijvers DM, Lemmens K, De Keulenaer GW, Martinet W, et al. Chronic intermittent mental stress promotes atherosclerotic plaque vulnerability, myocardial infarction and sudden death in mice. Atherosclerosis. Elsevier Ltd; 2015 Sep;242(1):288-94.

75. Haghighat A, Weiss D, Whalin MK, Cowan DP, Taylor WR. Granulocyte colony-stimulating factor and granulocyte macrophage colony-stimulating factor exacerbate atherosclerosis in apolipoprotein Edeficient mice. Circulation. 2007 Apr 17;115(15):2049-54.

76. Tanaka K, Nagata D, Hirata Y, Tabata Y, Nagai R, Sata M. Augmented angiogenesis in adventitia promotes growth of atherosclerotic plaque in apolipoprotein E-deficient mice. Atherosclerosis. Elsevier Ireland Ltd; 2011 Apr;215(2):366-73.

77. Lucerna M, Zernecke A, de Nooijer R, de Jager SC, Bot I, van der Lans C, et al. Vascular endothelial growth factor-A induces plaque expansion in ApoE knock-out mice by promoting de novo leukocyte recruitment. Blood. 2007 Jan 1;109(1):122-9.

78. Leppänen $\mathrm{P}$, Koota S, Kholová I, Koponen J, Fieber C, Eriksson U, et al. Gene transfers of vascular endothelial growth factor-A, vascular endothelial growth factor-B, vascular endothelial growth factor-C, and vascular endothelial growth factor-D have no effects on atherosclerosis in hypercholesterolemic low-density lipoprotein. Circulation. 2005 Aug 30;112(9):1347-52.

79. Khurana R, Zhuang Z, Bhardwaj S, Murakami M, De Muinck E, Yla-Herttuala S, et al. Angiogenesisdependent and independent phases of intimal hyperplasia. Circulation. 2004 Oct 19;110(16):2436-43.

80. Giannarelli C, Alique M, Rodriguez DT, Yang DK, Jeong D, Calcagno C, et al. Alternatively spliced tissue factor promotes plaque angiogenesis through the activation of hypoxia-inducible factor- $1 \alpha$ and vascular endothelial growth factor signaling. Circulation. 2014 Oct 7;130(15):1274-86.

81. Bhardwaj S, Roy H, Babu M, Shibuya M, Yla-Herttuala S. Adventitial gene transfer of VEGFR-2 specific VEGF-E chimera induces MCP-1 expression in vascular smooth muscle cells and enhances neointimal formation. Atherosclerosis. Elsevier Ireland Ltd; 2011 Nov;219(1):84-91.

82. Bhardwaj S, Roy H, Kärpänen T, He Y, Hi Y, Jauhiainen S, et al. Periadventitial angiopoietin-1 gene transfer induces angiogenesis in rabbit carotid arteries. Gene Ther. 2005 Mar;12(5):388-94.

83. Bhardwaj S, Roy H, Heikura T, Ylä-Herttuala S. VEGF-A, VEGF-D and VEGF-D(DeltaNDeltaC) induced intimal hyperplasia in carotid arteries. Eur J Clin Invest. 2005 Nov;35(11):669-76.

84. Sluimer JC, Kolodgie FD, Bijnens APJJ, Maxfield K, Pacheco E, Kutys B, et al. Thin-walled microvessels in human coronary atherosclerotic plaques show incomplete endothelial junctions relevance of compromised structural integrity for intraplaque microvascular leakage. J Am Coll Cardiol. 2009 Apr 28;53(17):1517-27.

85. Carmeliet P. Angiogenesis in health and disease. Nat Med. 2003 Jun;9(6):653-60.

86. Eriksson EE. Intravital microscopy on atherosclerosis in apolipoprotein e-deficient mice establishes microvessels as major entry pathways for leukocytes to advanced lesions. Circulation. 2011 Nov 8;124(19):2129-38. 
87. Carmeliet P, Jain RK. Molecular mechanisms and clinical applications of angiogenesis. Nature. 2011 May 19;473(7347):298-307.

88. Dvorak HF, Sioussat TM, Brown LF, Berse B, Nagy JA, Sotrel A, et al. Distribution of vascular permeability factor (vascular endothelial growth factor) in tumors: concentration in tumor blood vessels. J Exp Med. 1991 Nov 1;174(5):1275-8.

89. Dunmore BJ, McCarthy MJ, Naylor AR, Brindle NPJ. Carotid plaque instability and ischemic symptoms are linked to immaturity of microvessels within plaques. J Vasc Surg. 2007 Jan;45(1):155-9.

90. Augustin HG, Koh GY, Thurston G, Alitalo K. Control of vascular morphogenesis and homeostasis through the angiopoietin-Tie system. Nat Rev Mol Cell Biol. 2009 Mar;10(3):165-77.

91. Benest A V, Kruse K, Savant S, Thomas M, Laib AM, Loos EK, et al. Angiopoietin-2 is critical for cytokineinduced vascular leakage. Parikh SM, editor. PLoS One. 2013 Aug 5;8(8):e70459.

92. Willems S, Vink A, Bot I, Quax PHA, de Borst GJ, de Vries J-PPM, et al. Mast cells in human carotid atherosclerotic plaques are associated with intraplaque microvessel density and the occurrence of future cardiovascular events. Eur Heart J. 2013 Dec;34(48):3699-706.

93. Moulton KS, Heller E, Konerding M a, Flynn E, Palinski W, Folkman J. Angiogenesis inhibitors endostatin or TNP-470 reduce intimal neovascularization and plaque growth in apolipoprotein E-deficient mice. Circulation. 1999 Apr 6;99(13):1726-32.

94. Herrmann J, Best PJ, Ritman EL, Holmes DR, Lerman LO, Lerman A. Chronic endothelin receptor antagonism prevents coronary vasa vasorum neovascularization in experimental hypercholesterolemia. J Am Coll Cardiol. 2002 May 1;39(9):1555-61.

95. Moulton KS, Vakili K, Zurakowski D, Soliman M, Butterfield C, Sylvin E, et al. Inhibition of plaque neovascularization reduces macrophage accumulation and progression of advanced atherosclerosis. Proc Natl Acad Sci U S A. 2003 Apr 15;100(8):4736-41.

96. Drinane M, Mollmark J, Zagorchev L, Moodie K, Sun B, Hall A, et al. The antiangiogenic activity of rPAI$1(23)$ inhibits vasa vasorum and growth of atherosclerotic plaque. Circ Res. 2009 Feb 13;104(3):337-45.

97. Kampschulte M, Gunkel I, Stieger P, Sedding DG, Brinkmann A, Ritman EL, et al. Thalidomide influences atherogenesis in aortas of ApoE(-/-)/LDLR (-/-) double knockout mice: a nano-CT study. Int J Cardiovasc Imaging. 2014 Apr;30(4):795-802.

98. Xu X, Mao W, Chai Y, Dai J, Chen Q, Wang L, et al. Angiogenesis Inhibitor, Endostar, Prevents Vasa Vasorum Neovascularization in a Swine Atherosclerosis Model. J Atheroscler Thromb. 2015 Oct 1;22(10):1100-12.

99. Bot I, Jukema JW, Lankhuizen IM, van Berkel TJC, Biessen EAL. Atorvastatin inhibits plaque development and adventitial neovascularization in ApoE deficient mice independent of plasma cholesterol levels. Atherosclerosis. Elsevier Ireland Ltd; 2011 Feb;214(2):295-300.

100. Gössl M, Herrmann J, Tang H, Versari D, Galili O, Mannheim D, et al. Prevention of vasa vasorum neovascularization attenuates early neointima formation in experimental hypercholesterolemia. Basic Res Cardiol. 2009 Nov;104(6):695-706.

101. Luttun A, Tjwa M, Carmeliet P. Placental growth factor (PIGF) and its receptor Flt-1 (VEGFR-1): novel therapeutic targets for angiogenic disorders. Ann N Y Acad Sci. 2002 Dec;979:80-93.

102. Stefanadis C, Toutouzas K, Stefanadi E, Lazaris A, Patsouris E, Kipshidze N. Inhibition of plaque neovascularization and intimal hyperplasia by specific targeting vascular endothelial growth factor with bevacizumab-eluting stent: an experimental study. Atherosclerosis. 2007 Dec;195(2):269-76.

103. Hauer AD, van Puijvelde GHM, Peterse N, de Vos P, van Weel V, van Wanrooij EJA, et al. Vaccination against VEGFR2 attenuates initiation and progression of atherosclerosis. Arterioscler Thromb Vasc Biol. 
2007 Sep;27(9):2050-7.

104. Petrovan RJ, Kaplan CD, Reisfeld RA, Curtiss LK. DNA vaccination against VEGF receptor 2 reduces atherosclerosis in LDL receptor-deficient mice. Arterioscler Thromb Vasc Biol. 2007 May;27(5):1095-100.

105. Hauer AD, Habets KLL, van Wanrooij EJA, de Vos P, Krueger J, Reisfeld RA, et al. Vaccination against TIE2 reduces atherosclerosis. Atherosclerosis. 2009 Jun;204(2):365-71.

106. Jain RK, Finn A V, Kolodgie FD, Gold HK, Virmani R. Antiangiogenic therapy for normalization of atherosclerotic plaque vasculature: a potential strategy for plaque stabilization. Nat Clin Pract Cardiovasc Med. 2007 Sep;4(9):491-502.

107. Lindblom P, Gerhardt H, Liebner S, Abramsson A, Enge M, Hellstrom M, et al. Endothelial PDGF-B retention is required for proper investment of pericytes in the microvessel wall. Genes Dev. 2003 Aug 1;17(15):1835-40. 
Chapter 1 


\section{Chapter 2}

\section{Reversal of hypoxia in murine atherosclerosis prevents necrotic core expansion by enhancing efferocytosis}

Marsch E*, Theelen TL*, Demandt JA, Jeurissen M, van Gink M, Verjans R, Janssen A, Cleutjens JP, Meex SJ, Donners MM, Haenen GR, Schalkwijk CG, Dubois L, Lambin P, Mallat Z, Gijbels MJ, Heemskerk JW, Fisher EA, Biessen EA, Janssen BJ, Daemen MJ, Sluimer JC

*Authors contributed equally Modified from Arterioscler Thromb Vasc Biol. 2014 Dec;34(12):2545-53. 


\section{ABSTRACT}

\section{Objective}

Advanced murine and human plaques are hypoxic, but it remains unclear whether plaque hypoxia is causally related to atherogenesis. Here, we tested the hypothesis that reversal of hypoxia in atherosclerotic plaques by breathing hyperoxic carbogen gas will prevent atherosclerosis.

\section{Approach and Results}

$\mathrm{LDLr}^{-/-}$mice were fed a western-type diet, exposed to carbogen $\left(95 \% \mathrm{O}_{2}, 5 \% \mathrm{CO}_{2}\right)$ or air and the effect on plaque hypoxia, size and phenotype was studied.

First, the hypoxic marker pimonidazole was detected in murine $\mathrm{LDLr}^{-/}$plaque macrophages from plaque initiation onwards. Second, the efficacy of breathing carbogen (90min, single exposure) was studied. Compared to air, carbogen increased arterial blood $\mathrm{pO}_{2} 5$-fold in $\mathrm{LDLr}^{-/}$mice, and reduced plaque hypoxia in advanced plaques of the aortic root $(-32 \%)$ and arch $(-84 \%)$. Finally, the effect of repeated carbogen exposure on progression of atherosclerosis was studied in $\mathrm{LDLr}^{-/-}$mice fed a high cholesterol diet for an initial 4 weeks, followed by 4 weeks of diet and carbogen or air (both $90 \mathrm{~min} /$ day). Carbogen reduced plaque hypoxia (-40\%), necrotic core size (-37\%), and TUNEL+ apoptotic cell content $(-50 \%)$ and increased efferocytosis of apoptotic cells by MAC $3+$ macrophages $(+36 \%)$ in advanced plaques of the aortic root. Plaque size, plasma cholesterol, hematopoiesis and systemic inflammation were unchanged. In vitro, hypoxia hampered efferocytosis by bone marrow-derived macrophages, which was dependent on the receptor MerTK.

\section{Conclusions}

Carbogen restored murine plaque oxygenation and prevented necrotic core expansion by enhancing efferocytosis, likely via MerTK. Thus, plaque hypoxia is causally related to necrotic core expansion. 


\section{INTRODUCTION}

In many pathophysiological conditions, reduced oxygen tension (hypoxia) is a known stimulus of inflammation, angiogenesis and apoptosis $(1,2)$. As the progression of atherosclerosis is driven by the very same processes (3-5), we recently investigated if hypoxia was present in atherosclerosis. Indeed, plaque hypoxia was detected specifically in macrophages of advanced human atherosclerotic lesions using administration of the hypoxia-specific marker pimonidazole (6), as well as in advanced rabbit and murine plaques (7-10). However, whether plaque hypoxia is an innocent bystander-effect of macrophage influx, or a driving force behind plaque progression is still unknown.

As the in vitro effects of hypoxia on macrophage function are pro-angiogenic, proinflammatory (11) and anti-fibrotic (12), we hypothesized that hypoxia in plaque macrophages is pro-atherogenic. Thus, reoxygenation would be expected to prevent plaque progression. An attractive strategy to achieve plaque reoxygenation may be the breathing of hyperoxic gas carbogen, composed of $95 \% \mathrm{O}_{2}$ and $5 \% \mathrm{CO}_{2}$, as this successfully alleviated tumor hypoxia (13-15). Hyperoxic carbogen will increase oxygen dissolved in blood, thus $\mathrm{pO}_{2}$, rather than enhancing already maximal hemoglobin saturation. Additionally, the $\mathrm{CO}_{2}$ component of carbogen contributes to increased oxygen supply: $5 \%$ $\mathrm{CO}_{2}$ ensures a respiratory drive, prevents vasodilation associated with $100 \% \mathrm{O}_{2}$ and shifts the hemoglobin- $\mathrm{O}_{2}$ dissociation curve towards facilitated oxygen delivery (16). Thus, oxygen blood content, oxygen delivery, and oxygen diffusion are vastly improved, and enhance tissue oxygenation. Therefore, we hypothesized that carbogen delivery will reverse hypoxia in murine atherosclerotic plaques, and thus reverse hypoxia-associated detrimental effects on plaque inflammation and progression. 


\section{MATERIALS AND METHODS}

\section{Animals}

Mouse experiments were approved by the regulatory authority of the Maastricht University Medical Center, and were performed in compliance with the Dutch government guidelines. All mice were bred at least $9 \mathrm{x}$ on C57/BI6 background. Male $\mathrm{LDLr}^{-/}$were obtained from an in-house breeding colony, originally derived from Charles River (Wilmington, MA, USA). Mer tyrosine kinase domain deficient $\left(\right.$ MerTK $\left.^{\mathrm{kd}}\right)(17,18)$ and CD36 knockout mice $\left(\mathrm{CD}^{-/-} 6^{-}\right.$(19) and wildtype control mice were previously described.

\section{Blood gas analyses}

An arterial catheter (20) was implanted in five $\mathrm{LDLr}^{-/}$mice on chow using isoflurane anesthesia (1.5-2\%) and peri-operative ibuprofen (s.c). The right femoral artery was exposed, and a heat-stretched polyethylene- 25 cannula was inserted $(1.5 \mathrm{~cm})$ and subcutaneously guided to the neck of the mouse. Here the catheter was fixed, extended, filled with heparinized saline $(10 \mathrm{U} / \mathrm{ml})$, and plugged. The mice were allowed to recover $24 \mathrm{hrs}$ before measurements were made. Mice were placed in induction chambers and the arterial line was extended through the outlet of the chamber to allow blood sampling without altering the continuous carbogen flow $(5 \mathrm{~L} / \mathrm{min}$, normobaric, NTG Sol). Arterial blood $(70 \mu \mathrm{l})$ was collected before and after 30 minutes of flow, and 5 minutes after flow cessation and analyzed within 5 minutes on a Chiron blood gas analyzer (Siemens).

\section{Atherogenesis and carbogen exposure}

First, hypoxia development in atherogenesis was investigated in $\mathrm{LDLr}^{-1-}$ mice ( $\mathrm{n}=5 /$ group) fed a $0.25 \%$ cholesterol diet (special diet services, \#824171, 15\% cocoa butter, $10 \%$ maize starch, $20 \%$ casein, $40.5 \%$ sucrose, $5.95 \%$ cellulose) for $0,4,8,12$ or 16 weeks. Secondly, acute reversal of plaque hypoxia was investigated in $\mathrm{LDLr}^{-/}$mice with advanced, hypoxic plaques ( $n=5 /$ group, 12 weeks of diet), which were subjected to a single 90 minute exposure of carbogen or air $\left(21 \% \mathrm{O}_{2}\right)$. Halfway during the exposure, mice were injected intraperitoneally (IP) with the hypoxia-specific marker pimonidazole $(100 \mathrm{mg} / \mathrm{kg}$, hypoxyprobe Omni kit, Hypoxyprobe Inc. Burlington, MA, USA) and sacrificed directly after cessation of carbogen flow. Thirdly, the effect of chronic carbogen exposure on atherogenesis was studied in thirty $\mathrm{LDLr}^{-/}$( $n=15 /$ group), fed a $0.25 \%$ cholesterol diet for four weeks. During the four additional weeks of diet, mice were exposed daily to 90 minutes of carbogen or air. Twenty-four hours after the last exposure, mice were injected with pimonidazole one hour prior to sacrifice.

\section{Atherosclerotic lesion quantification and immunohistochemistry}

Mice were euthanized using a pentobarbital overdose $(100 \mathrm{mg} / \mathrm{kg})$ and blood was collected via the left ventricle for flow cytometry, absolute white blood cell counts (Coulter counter AC.T diff) and total plasma cholesterol analysis. Aortic roots, arches and organs were excised and fixed in $1 \%$ paraformaldehyde. In additional $\mathrm{LDLr}^{-/}$mice, white blood cells were collected from the abdominal aorta and the saphenous vein, and fixed (1\% paraformaldehyde, 2 hours) following erythrocyte lysis in iso-osmotic ammonium 
chloride solution. Remaining white blood cells were mixed with agar and paraffinembedded.

Aortic roots and arches were serially sectioned, and stained with hematoxylin and eosin (HE, Sigma) for blinded quantification of the lesion areas using computerized morphometry (Leica QWin V3, Cambridge, UK). Total plaque area and lipid core content were obtained by averaging measurements of five representative $(\mathrm{HE})$ sections $(25 \mu \mathrm{m}$ apart) of the aortic root and aortic arch. In the aortic arch, plaque size was quantified as the sum of the inner and outer curvature plaques, as well as all three major branches.

Necrotic core was defined as a-cellular and a-nuclear plaque area containing cholesterol clefts.

Atherosclerotic plaque size and necrotic core area in the aortic arch was determined in 5 representative HE stainings.

Hypoxia was detected in atherosclerotic plaques (aortic root, aortic arch), murine kidney (positive control), liver, spleen, lymph nodes, thymus and white blood cells. In vivo, pimonidazole is irreversibly metabolized in living, hypoxic cells $\left(\mathrm{pO}_{2} \leq 10 \mathrm{mmHg} \sim 1 \% \mathrm{O}_{2}\right)(21)$, and metabolites form adducts onto thiol-containing proteins. These epitopes were ex vivo detected by immunohistochemistry with a rabbit polyclonal antibody (clone 2627) that only recognizes hypoxic metabolites derivatives of pimonidazole (22). Hypoxia was quantified in images of entire organs or plaques (\% pimonidazole area/total tissue area). Atherosclerotic plaques were further characterized for macrophage size and content $\left(\mathrm{MAC3}^{+}\right.$cells/total cells and $\mathrm{MAC3}^{+}$cells/plaque area, BD), T-cells (CD3 ${ }^{+}$cells/plaque area, Miltenyi), collagen (picosirius red area/plaque area, analyzed with polarized light microscopy), proliferating cells ( $K i 67^{+}$cells/plaque area, Abcam), tissue factor (TF) $\left(\mathrm{TF}^{+}\right.$ cells/plaque area, Santa Cruz), M1 inducible nitric oxide synthase (iNOS ${ }^{+}$cells/plaque area, Abcam), M2 mannose receptor ( $\mathrm{MR}^{+}$cells/adventitia, Abcam) expression and 8-hydroxy2'deoxy-guanosine $\left(8 \mathrm{OH}-\mathrm{dG}\right.$, Bioconnect) for oxidative stress damage $\left(8 \mathrm{OH}-\mathrm{dG}^{+}\right.$ cells/plaque area, Japan institute for the control of aging). Apoptosis (TUNEL ${ }^{+}$ nuclei/plaque area, Roche) and clearance of apoptotic cells (efferocytosis) in situ were analyzed in entire tissue sections using TUNEL/MAC3 double immunohistochemistry as the ratio of bound/free apoptotic cells according to established methods $(23,24)$.

\section{Multispectral image analysis}

Multispectral imaging was used to quantitatively measure colocalization of individual immunohistochemical staining products in tissue stained with multiple antibodies. Sections stained with pimonidazole and MAC3 were analyzed with the Nuance spectral imaging system (Perkin Elmer/Caliper Life Sciences, Hopkinton, MA, USA). Spectral imaging data sets were taken at $420-720 \mathrm{~nm}$ at $20 \mathrm{~nm}$ intervals using a DM-5000 Leica microscope system at 20x (plan apo). Spectral libraries of single-red (Vector Red), singleblue (Vector Blue) were obtained from the control slides. The resulting library was applied to the double stained slides to spectrally segregate them into individual component images using the Nuance ${ }^{\mathrm{TM}} 3.0$ software. Pseudo-colors were assigned to unmixed images, and composite images showing co-localization were generated with the Nuance 3.0 software. Quantitative assessment of co-localization and pixel-based measurement of the individual markers per microscopic field was done with the same software. 


\section{Total plasma cholesterol, triglyceride, antioxidant capacity and cytokines}

Plasma was separated by centrifugation, and stored at $-80^{\circ} \mathrm{C}$ until further use. Standard enzymatic techniques were used for the assessment of plasma cholesterol (product no. 07-3663-5, Roche, Almere, the Netherlands) and plasma triglycerides (FS5' Ecoline REF 1 57609990 314; DiaSys - Diagnostic Systems GmbH, Holzheim, Germany) automated on the Cobas Fara centrifugal analyzer (Roche).

Anti-oxidant capacity of plasma was determined using the trolox equivalent antioxidant capacity (TEAC) assay, as described previously (25). In short, plasma was deproteinized with a final concentration of 5\% TCA and incubated with an ABTS radical solution $\left(2,2^{\prime}-\right.$ azino-bis(3-ethylbenzthiazoline-6-sulfonic acid) diammonium salt) for $5 \mathrm{~min}$. Subsequently, the reduction in absorbance at $734 \mathrm{~nm}$ was quantified. This was normalized to trolox control, a water-soluble vitamin $\mathrm{E}$ analogue. The assay reflects the amount of scavenged radicals expressed as $\mu \mathrm{M}$ trolox equivalents.

Additionally, murine Interleukins (IL) $2,4,5,6,10,12$ p70 and $1 \beta$, Interferon $\gamma$ (IFN- $\gamma$ ), chemokine (C-X-C motif) ligand 1 (CXCL1, KC) and Tumor Necrosis Factor $\alpha$ (TNF- $\alpha$ ) were measured in plasma using a multispot sandwich immunoassay (V-plex pro-inflammatory panel 1, Meso Scale Discovery, Gaithersburg, USA), according to manufacturers' instruction.

\section{Flow cytometry analysis}

Flow cytometry analysis was performed on cells isolated from blood, spleen, lymph nodes, and bone marrow ( $n=10 /$ group). Spleens and lymph nodes were dissociated into singlecell suspensions, and enzymatically digested for dendritic cell separation using liberase and DNAse (both $0.2 \mathrm{mg} / \mathrm{ml}$, Roche) for 30 minutes in RPMI medium. Blood and spleen were subjected to erythrocyte lysis. Staining was performed using combinations of specific antibodies to detect bone marrow stem cells (lin/Sca-1/c-kit; eBioscience, Bd), common myeloid progenitors (CD16/32 ${ }^{\text {int }}, C D 34^{\text {int; }}$; eBioscience, $B D$ respectively), granulocytemacrophage progenitors $\left(C D 16 / 32^{\text {high }}, C D 34^{\text {high }}\right)$, erythrocyte-megakaryocyte progenitors (CD16/32- CD34-), monocytes (CD11 b high $^{-}$Ly6G-, Ly6C high/int/low; BD, eBioscience, Miltenyi respectively), granulocytes $\left(C D 11 b^{\text {high }}\right.$, Ly6G $\left.^{\text {high }}\right), T$ cells $\left(C D 3 \varepsilon^{+}\right.$; Miltenyi), T helper cells $\left(\mathrm{CD} 4^{+} ; \mathrm{BD}\right)$, cytotoxic T cells $\left(\mathrm{CD} 8 \mathrm{a}^{+} ; \mathrm{BD}\right)$, effector/memory T cells $\left(\mathrm{CD} 44^{\text {high }}, \mathrm{CD} 62^{\text {low }} ; \mathrm{BD}\right.$, eBioscience respectively), naïve T cells (CD44 ${ }^{\text {low }}, C D 62^{\text {high }}$ ), regulatory $T$ cells $\left(C D 4^{+}, C D 25^{+}\right.$, FoxP3 ${ }^{+}$; eBioscience), B cells (B220+; BD), CDC (CD11 $c^{\text {high }}$, eBioscience), pDC (CD11 $c^{\text {mid }}$, $\mathrm{B} 220^{+}, \mathrm{Ly}_{6 \mathrm{C}^{+}}$), and NK cells (NK1.1 ${ }^{+}$; eBioscience). Data were acquired and analyzed using a FACSCanto II and FACSdiva software (BD Bioscience).

\section{Cell culture}

Bone marrow was isolated and cells were cultured for 7 days in RPMI-1640 (Gibco with Glutamax, 2g/L glucose) supplemented with 10\% FCS, 100U/ml Penicillin-Streptomycin, and 15\% L929-conditioned medium to generate bone marrow-derived macrophages (BMDM). Jurkat T cells and $\mathbf{J 7 7 4}$ murine macrophages were cultured in RPMI-1640 supplemented with $10 \%$ FCS, $100 \mathrm{U} / \mathrm{ml}$ Penicillin Streptomycin.

Human THP-1 cells were obtained from the American Type Culture Collection (ATCC 10801, Manassas, VA). Cells were grown in RPMI 1640 medium (Gibco-Invitrogen, Grand Island, NY, USA) containing 10\% FetalClone ${ }^{\circledR}$ III (Hyclone, Pe-bio, Hogan, UT, USA) (v/v), 1\% GlutaMAXTM I (w/v) (Gibco-Invitrogen, Grand Island, NY, USA), 125 mM Hepes, penicillin 
(100 units/ml), and streptomycin $(100 \mu \mathrm{g} / \mathrm{ml})$ (Gibco-Invitrogen, Grand Island, NY, USA) at $37^{\circ} \mathrm{C}$ in a humidified incubator with a $5 \% \mathrm{CO}_{2}$ atmosphere. To differentiate THP-1 cells into macrophages, cells were seeded at a density of $1 \times 106$ cells $/ \mathrm{ml}$ and incubated for 72 hours with $0.2 \mu \mathrm{M}$ phorbol 12-myristate 13-acetate (PMA, Sigma, Saint Louis, MO, USA).

Jurkat $\mathrm{T}$ cells were labeled with calcein-AM $(1 \mu \mathrm{g} / \mathrm{ml}$ Invitrogen) prior to induction of apoptosis by UV irradiation (15 $\mathrm{min} 254 \mathrm{~nm}$, UVS-26, $6 \mathrm{~W}$ bulb $0.02 \mathrm{~J} / \mathrm{s} / \mathrm{cm}_{2}$ ) and added 3:1 to $\mathrm{J} 774$ or BMDM. Macrophages were exposed to $21 \%$ or $0.2 \%$ O2 (Invivo2 1000, Ruskinn technology LTD, Pencoed, UK) during $45 \mathrm{~min}$ of efferocytosis. After thorough washing, macrophages were dissociated and analyzed using FACS for the percentage of calcein/jurkat $^{+}$macrophages. Twenty-four hours prior to efferocytosis, BMDM were

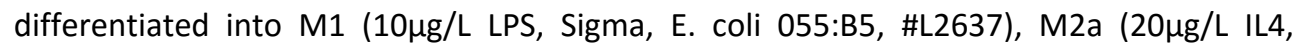

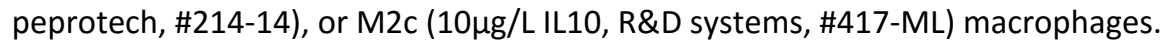

To analyze apoptosis, BMDM or $\mathrm{J} 774$ were exposed to hypoxia $\left(0.2 \% \mathrm{O}_{2}\right)$ for $24 \mathrm{hrs}$ or UVirradiated for $15 \mathrm{~min}$ and apoptotic cells detected using annexin A5-Alexa594 (Invitrogen) on microscopic images. Additionally, BMDMs were cultured in normoxia or hypoxia with 7 ketocholesterol $(25 \mu \mathrm{M}$, Sigma), tunicamycin $(2.5 \mu \mathrm{g} / \mathrm{ml}$, Sigma) or oxLDL $(25 \mu \mathrm{g} / \mathrm{ml})$ supplementation for $24 \mathrm{hrs}$ and apoptotic cells were detected using TUNEL (Roche).

Pimonidazole specificity was confirmed in bone marrow derived macrophages treated with $25 \mu \mathrm{g} / \mathrm{ml}$ oxLDL, LDL or control for $24 \mathrm{~h}$. Foam cell formation was confirmed by OilRed-O staining. Subsequently, cells were supplemented with pimonidazole $(100 \mu \mathrm{M}$,$) and$ exposed to $0,0.2,1,5$ or $20 \% \mathrm{O}_{2}$ for $0 \mathrm{~h}, 1 \mathrm{~h}, 4 \mathrm{~h}$ or $16 \mathrm{~h}$. Cells were subsequently fixed, stained with anti-pimonidazole-FITC and geomean fluorescence was acquired and analyzed using a FACSCanto II and FACSdiva software (BD Bioscience).

ROS production by BMDMs cultured in $21 \%$ or $0.2 \%$ oxygen for 24 hours was measured using the cell permeant reagent $2^{\prime}, 7^{\prime}$-dichlorofluorescin diacetate (DCFDA, 10 $\mu \mathrm{M}$, Invitrogen, 488). Percent DCFDA positive cells were assessed via flow cytometry and $\mathrm{H}_{2} \mathrm{O}_{2}$ treated BMDMs in normoxia $(100 \mu \mathrm{M}, 10 \mathrm{~min})$ served as positive control.

\section{Quantitative RT- PCR (QPCR)}

In vitro experiments for gene expression analysis were performed in quadruplicate, and repeated twice. Total RNA was isolated and transcribed as described (26). QPCR analyses were performed from $10 \mathrm{ng}$ CDNA using SYBR green and gene specific primer sets (supplemental table 1). Two housekeeping genes (cyclophilin, 18S rRNA) insensitive to changes in $\mathrm{O} 2$ were used to correct for differences in mRNA levels between samples.

\section{Statistical analysis}

All data are presented as mean \pm SEM. Blood gas parameters were analyzed using pairedsample testing, while independent-sample tests were done for all other parameters. Following a Shapiro-Wilk normality test, two groups were compared with student's t-test or Mann-Whitney rank-sum test. Parameters with more than two groups were analyzed using Kruskal-Wallis rank-sum test, followed by Dunn's post-hoc testing (GraphPad Prism4). Correlations between MAC3, plaque area and necrotic core with hypoxia (pimonidazole) were analyzed using univariate and multivariate linear regression (IBM SPSS Statistics 20). 


\section{RESULTS}

\section{Murine plaque macrophages are hypoxic, independent of plaque stage}

Low density lipoprotein receptor ( $\left.\mathrm{LLLr}^{--}\right)$mice were fed a Western type diet (WTD) for 0 16 weeks to monitor the onset and distribution of plaque hypoxia with pimonidazole at different plaque stages in the aortic root (Figure $1 \mathrm{~A}$ ).

First, specificity of pimonidazole and its detection antibody was confirmed in mice injected with vehicle or pimonidazole (Supplemental Figure 1A). Plaque hypoxia colocalized mainly with MAC3+ macrophages and foam cells shown by multispectral analysis of double immunohistochemistry (Figure 1C-D). Pimonidazole detection in cultured bone marrow derived macrophages (BMDM) sharply increased below $1 \% \mathrm{O}_{2}(\sim 10 \mathrm{mmHg})$ (Supplemental Figure $1 \mathrm{~B}-\mathrm{C}$ ), confirming previous data (21). Also, macrophage lipid loading per se did not result in pimonidazole positivity (Supplemental Figure $1 \mathrm{D}$ ). Earlier studies have also shown that oxidative stress exposure does not affect pimonidazole detection (6), overall suggesting that pimonidazole staining truly marks hypoxic macrophages.

Plaque hypoxia may either arise from reduced oxygen supply to the growing plaque or from increased oxygen demand by inflammatory cells. A strong correlation between MAC3 content and pimonidazole positivity supports the latter explanation, while plaque size was not a determinant for plaque hypoxia (Figure 1E). A slight reduction in macrophage content after 16 weeks was mirrored by a reduction in hypoxia (Figure 1A, D). Thus, murine plaque hypoxia is dictated by macrophage content, not by plaque thickness and oxygen supply. Indeed, pimonidazole was detected directly adjacent to the luminal arterial oxygen supply, in small fatty streaks after 4 weeks WTD, while being absent in the media of diseased and non-diseased arteries (Figure 1A). Although pimonidazole is metabolized by living cells only, reflected by a significant univariate correlation of plaque necrosis and hypoxia, necrosis was not a significant predictor in multivariate regression (figure $1 \mathrm{~A}, \mathrm{E}$ ). In addition, tissue-resident macrophages in inflammation-rich fatty livers and lymphoid organs of WTD-fed LDLr-/- mice were hypoxic (Supplemental Figure $2 \mathrm{~A}-\mathrm{C}$ ). As even venous pO2 is 4-fold higher than the $10 \mathrm{mmHg}$ detection threshold of pimonidazole (21), arterial and venous white blood cells were negative for pimonidazole (Supplemental Figure 2 D), as expected.

In conclusion, tissue resident and plaque macrophages are hypoxic. Plaque macrophages remain the only predictor of plaque hypoxia after multivariate regression, not plaque size or necrotic core content. 
A

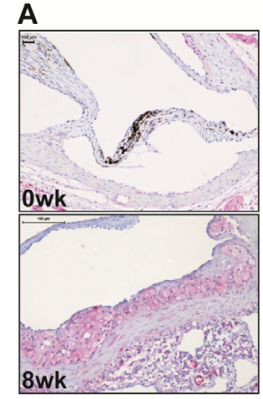

C

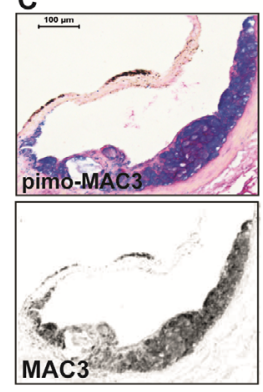

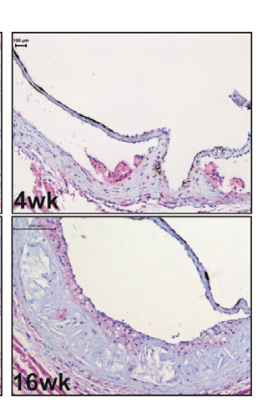

B

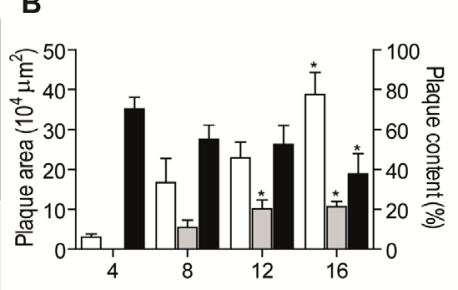

weeks of WTD

\section{Plaque area} Macrophage

D

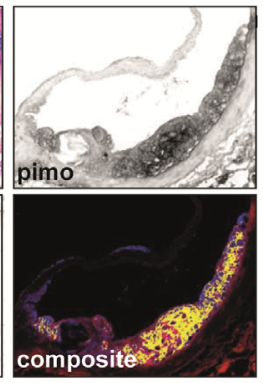

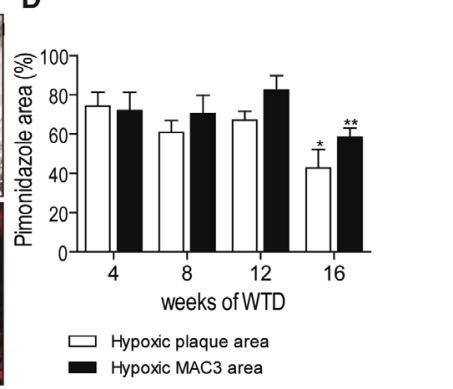

$\mathrm{E}$

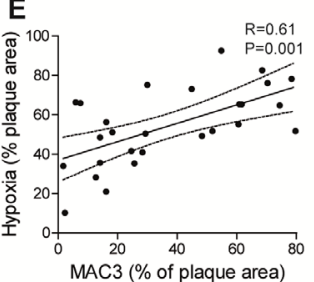

MAC3 (\% of plaque area)
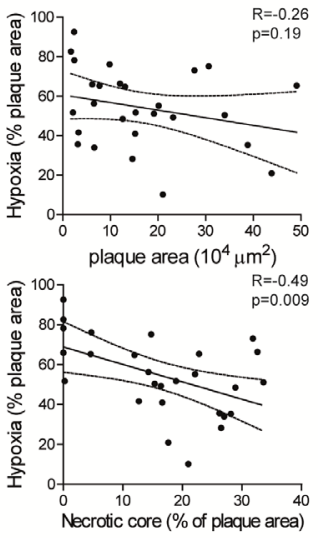

Figure 1. Murine plaque macrophages are hypoxic, independent of plaque stage

(A) Representative pictures of pimonidazole-stained aortic root (red) from LDLr ${ }^{-/}$mice after $0,4,8$ and 16 weeks of WTD ( $n=5 /$ group) and (B) quantification of plaque area (left $y$-axis), necrotic core (right $y$-axis) and macrophage content (right $y$-axis), ${ }^{*} p$-value $<0.05$ vs. 4 weeks. (C) Illustration of principle multispectral analysis in aortic roots from LDLr/- mice with advanced plaque, double-stained with pimonidazole (Pimo, red) and MAC3 (blue), respectively. (D) Quantification of plaque hypoxia and hypoxic macrophages (MAC3, pimonidazole double positive) in LDLr ${ }^{-/}$aortic root ( $n=5 /$ time point). ${ }^{*}$ p-value $<0.054$ vs. 16 weeks, ${ }^{* *}$-value $<0.0112$ vs. 16 weeks. (E) Scatter plots of plaque hypoxia with MAC3 (top panel), plaque area (middle panel), or necrotic core area (lower panel) with univariate linear regression line and $95 \%$ confidence interval (dashed lines). Data from LDLr- mice fed WTD for 4, 8, 12 and 16 weeks ( $n=5$ per time point $+n=15$ air breathing controls. $n=35$ in total).

\section{Carbogen exposure increased arterial $\mathrm{pO}_{2}$ and alleviated plaque hypoxia}

Carbogen significantly increased arterial $\mathrm{pO}_{2}$ in awake $\mathrm{LDLr}^{-/}$mice (Figure 2A). Simultaneously, blood lactate was drastically reduced, while $\mathrm{pCO}_{2}$ was slightly elevated (Figure $2 \mathrm{~A}$ ), and $\mathrm{pH}$, glucose and electrolytes (data not shown) unchanged. Within minutes after cessation of carbogen breathing, all blood levels, except for lactate, returned to baseline (data not shown).

As carbogen successfully increased arterial $\mathrm{pO}_{2}$, its effect on plaque hypoxia was studied. Single 90 minute carbogen exposure in $\mathrm{LDLr}^{-/}$mice with advanced, hypoxic plaques led to a dramatic reduction of plaque hypoxia in the aortic arch and root compared to similarly sized plaques in mice breathing air (Figure 2B, C). Macrophage content, the main predictor of plaque hypoxia, was similar in both groups (Figure 2B, C). Likewise, repeated carbogen exposure was able to alleviate plaque hypoxia in $\mathrm{LDLr}^{-1-}$ mice, which were fed a WTD for an initial 4 weeks followed by 4 weeks of diet and carbogen or air exposure (both $90 \mathrm{~min} /$ day). Hypoxia decreased with $50 \%$ in the aortic arch and $42 \%$ in the root compared to control (Figure 2D,E), while macrophage content was not affected by carbogen gas (Figure 2D-F). Moreover, carbogen was able to significantly reoxygenate liver and spleen (Supplemental Figure $2 \mathrm{~A}-\mathrm{C}$ ). 
To prevent oxygen toxicity possibly associated with long-term carbogen exposure, carbogen exposure was limited to 4 weeks. Chronic carbogen exposure did not affect plasma cholesterol, triglycerides, hemoglobin, or hematocrit, nor erythropoiesis, or myelopoiesis as shown by flow cytometry analysis (27) of stem cells and myeloid progenitors in bone marrow. Also it did not impact monocyte, granulocyte, T cell, B cell, and dendritic cell numbers in blood, spleen and lymph node (Supplemental Figure 3).

A
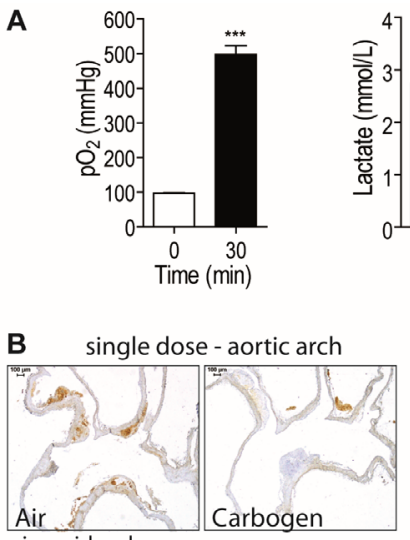

pimonidazole
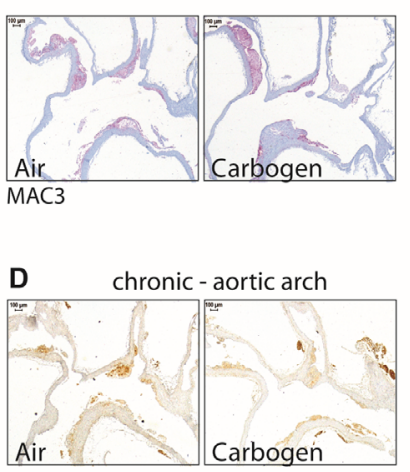

pimonidazole

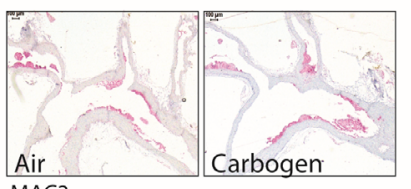

MAC3
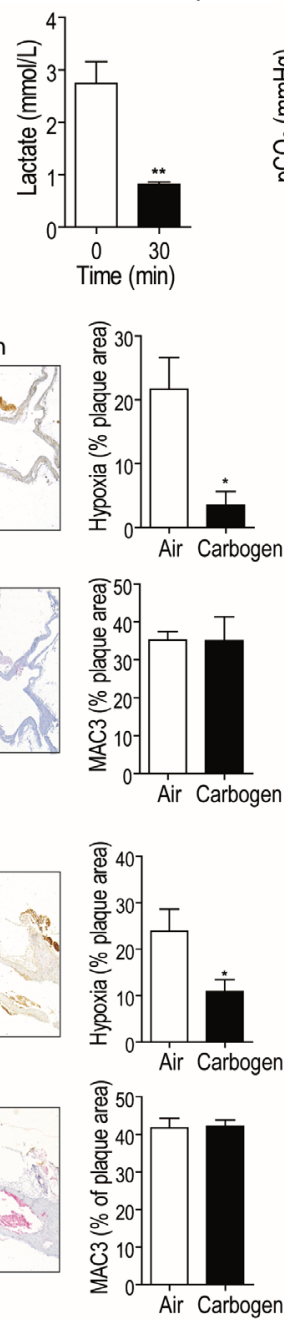
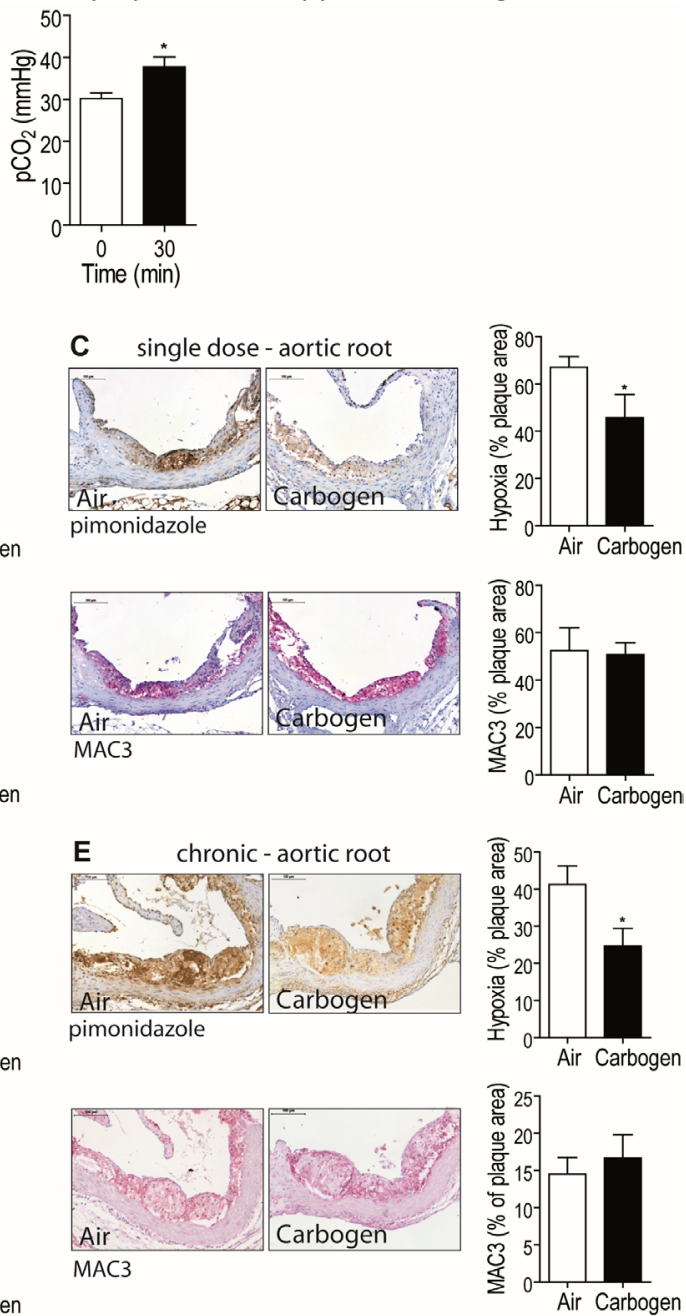

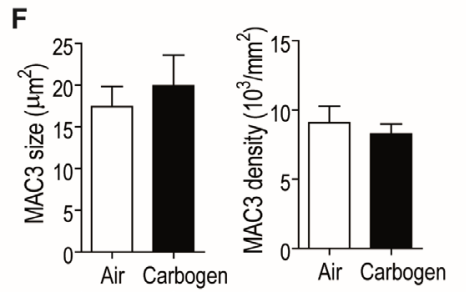

Figure 2. Carbogen increased arterial $\mathrm{pO}_{2}$ and alleviated plaque hypoxia

(A) Arterial $\mathrm{pO}_{2}$ (left panel), lactate concentration (middle panel) and $\mathrm{pCO}_{2}$ (right panel) before and after 30 minutes of carbogen exposure in $\mathrm{LDLr}^{-/}$mice on chow, ${ }^{* * *} \mathrm{p}$-value $<0.001,{ }^{* *} \mathrm{p}$-value $<0.01$ vs. before exposure. 36 
(B) Representative pictures and quantification of pimonidazole (brown) and MAC3 (red) immunohistochemistry in aortic arch and branches of $\mathrm{LDLr}^{-/}$mice exposed to single dose of air or carbogen. (C) Representative pictures and quantification of plaque hypoxia and macrophages in the aortic root upon single dose of air or carbogen. (D) Representative pictures and quantification of plaque hypoxia and macrophages in the aortic arch and (E) root of $\mathrm{LDLr}^{-1-}$ mice after chronic carbogen or air treatment. *p-value $<0.05$ vs. air. (F) Quantification of macrophage size (left graph) and density (right graph) upon air or carbogen treatment.

\section{Plaque reoxygenation improved efferocytosis and apoptosis in vivo}

Notably, plaque reoxygenation through daily carbogen exposure was able to prevent necrotic core expansion by $37 \%$ in advanced plaques of the aortic root compared to control, while plaque size was not altered (Figure 3A). Necrotic core expansion is determined by the balance between apoptosis and the clearance of apoptotic cells by phagocytes, a process called efferocytosis. Efferocytosis was quantified in situ, as the ratio of macrophage-bound vs. free apoptotic cells in a TUNEL/MAC3 double immunohistochemistry $(23,24)$. Indeed, carbogen exposure reduced the density of apoptotic cells in the plaque in situ by $50 \%$ and improved efferocytosis of apoptotic cells by $\mathrm{MAC3}^{+}$-macrophages by almost $40 \%$ compared to air (Figure 3B,C). Increased efferocytosis was seen despite similar plaque macrophage content, size and density (Figure 2E, F). Intraplaque proliferation, collagen content, T cell and total cell density as well as tissue factor (TF) expression as a measure of thrombogenicity (28) were unaffected by carbogen (Figure 3E-H).

In initial plaques of the aortic arch, carbogen did not affect plaque size or macrophage content (Supplemental Figure 4), despite successful reoxygenation (Figure 2D). 
A
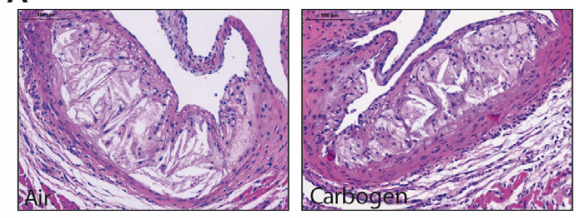

B

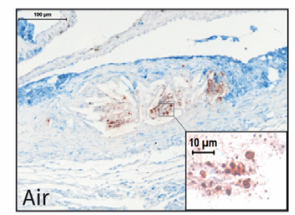

D

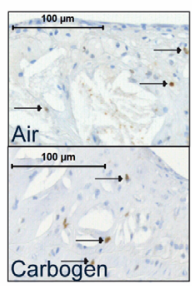

$\mathbf{F}$

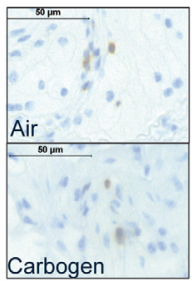

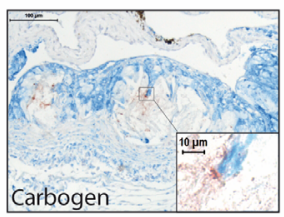

E
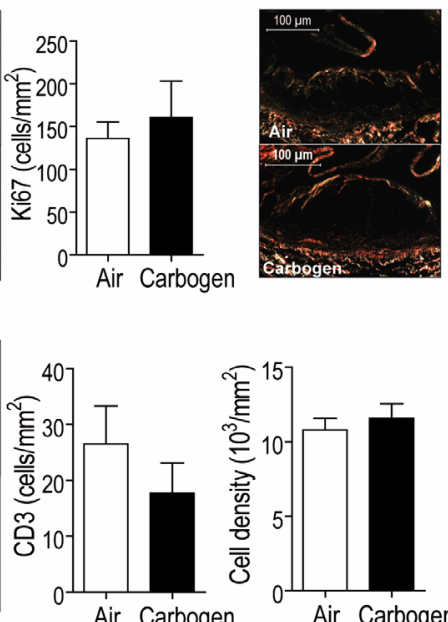
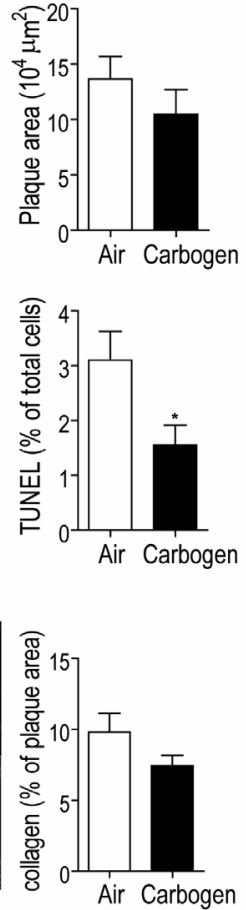

G

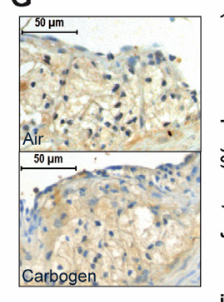

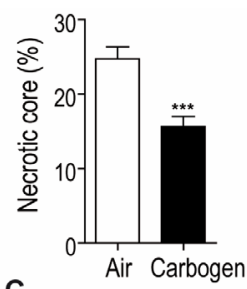

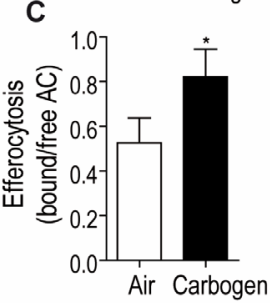

Figure 3. Plaque reoxygenation prevented necrotic core expansion and stimulated efferocytosis in vivo

(A) Hematoxylin and eosin staining and quantifications of aortic root plaque size (left graph) and necrotic core content (right graph) of male LDLr ${ }^{-/}$mice treated with air or carbogen and fed a WTD for 8 weeks. ${ }^{* * *} \mathrm{p}$ value $<0.001$ vs. air. (B) TUNEL and MAC3 double immunohistochemistry and weak counterstain with hematoxylin (1:50) of LDLr-/ aortic roots treated with air or carbogen. Insets show free TUNEL + nuclei (brick red) in air treated group, while TUNEL+ nuclei were associated with macrophages (blue) in carbogen treated group. Graphs represent quantification of apoptosis, and (C) efferocytosis (ratio MAC3-bound/free TUNEL+ cells), *p-value $<0.05$ vs. air (D). Quantification of proliferation (Ki67) expression (E) collagen content, (F) T cell density and total cell density, as well as (G) tissue factor expression, as a measure for thrombogenicity, in aortic roots of $\mathrm{LDLr}^{-1}$ mice after chronic air or carbogen exposure (left graph) and in BMDM exposed to 21 or $0.2 \% \mathrm{O}_{2}$ (right graph). 


\section{Neither ROS formation, nor macrophage polarization explain hypoxic repression of efferocytosis}

Generation of reactive oxygen species (ROS) during carbogen-induced reoxygenation might be involved in apoptosis or efferocytosis. However, neither ROS-mediated DNA damage, analyzed by 8-hydroxy-2'deoxy-guanosine ( $8 \mathrm{OH}-\mathrm{dG})$ immunohistochemistry, nor anti-oxidant capacity of the plasma was changed after chronic (Figure 4A) and single carbogen exposure (not shown). Additionally, hypoxia did not alter macrophage ROS activity in vitro (Figure 4A).

Apoptosis and efferocytosis were further studied in vitro. Chronic hypoxia $\left(0.2 \% \mathrm{O}_{2}\right)$ did not stimulate apoptosis in murine primary BMDM (Figure 4B) or $\mathrm{J774}$ (data not shown) as single stimulus, nor augment apoptosis of additional ER-stress stimuli such as 7ketocholesterol or tunicamycin. In fact, cells even seemed to be protected from ER-stress mediated apoptosis (Figure 4B). Efferocytosis of labeled, apoptotic Jurkat T-cells by both the $\mathbf{J 7 7 4}$ murine macrophage cell line and BMDM was hampered in hypoxia $\left(0.2 \% \mathrm{O}_{2}\right)$ compared to $21 \% \mathrm{O}_{2}$ control (Figure $4 \mathrm{C}$ ). Thus, hypoxia hampered efferocytosis in vitro.

Since anti-inflammatory $\mathrm{M} 2 \mathrm{c}$ macrophages are thought to be more efficient in phagocytosis/efferocytosis (29), the effect of hypoxia on polarization was studied. Hypoxia reduced M2 gene expression (interleukin 10 (IL-10) and mannose receptor (MR)), whereas M1 genes (IL-6 and inducible nitric oxide synthase) were strongly upregulated (Figure 4D). In vivo, unlike iNOS, $\mathrm{MR}^{+}$macrophages were hardly present in the plaque; therefore adventitial $\mathrm{MR}^{+}$cells were quantified. A trend towards less $\mathrm{M} 1$ in the plaque and more M2 expression in the adventitia of carbogen treated mice was found (Figure 4E-F). Additionally, plasma cytokine levels were essentially not changed, with a trend towards less IL-6 in the carbogen treated group (Figure 4G).

Next, it was investigated if M2 polarization prior to hypoxia could rescue hypoxiamediated repression of efferocytosis. Under normoxic conditions $\mathrm{M} 2 \mathrm{c}$ polarization enhanced macrophage efferocytosis capacity compared to unpolarized BMDM, and, unexpectedly, so did M1 polarization (Figure $4 \mathrm{H}$ ). However, polarization to M2c or M1 was not able to restore the repression of efferocytosis by hypoxia, suggesting that macrophage polarization is not involved in oxygen-dependent regulation of efferocytosis. 

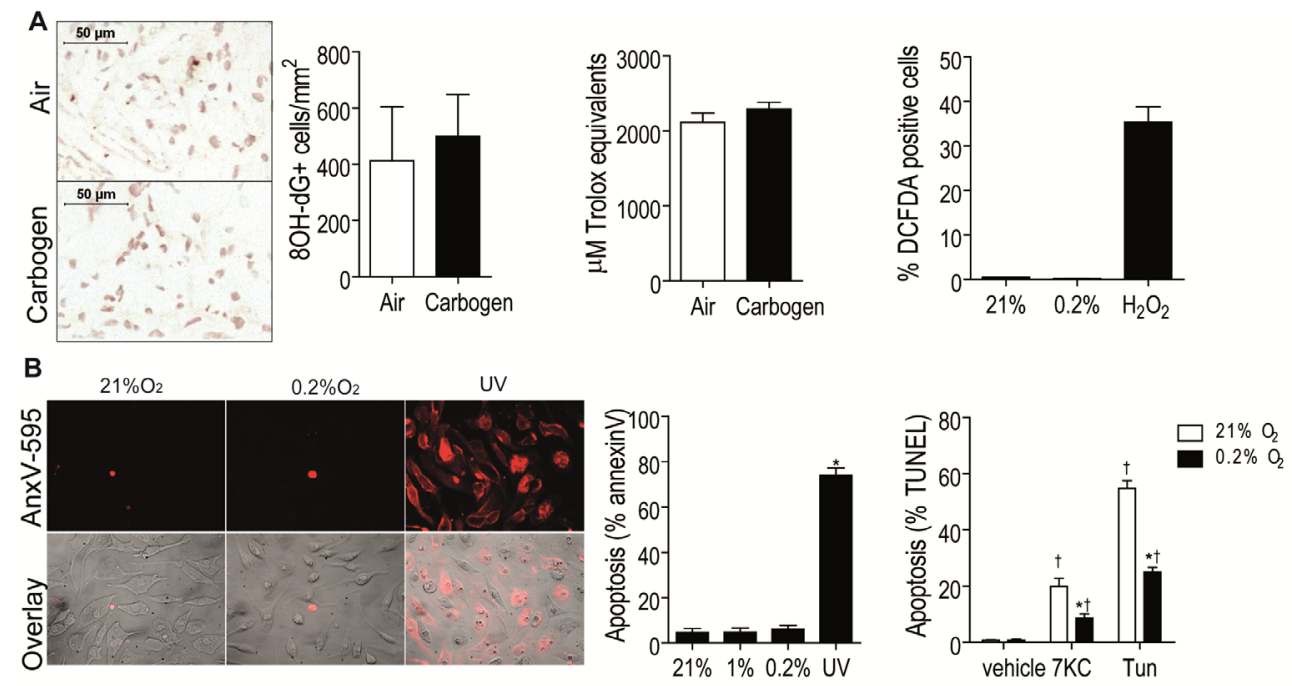

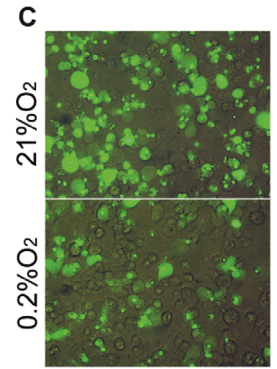

E
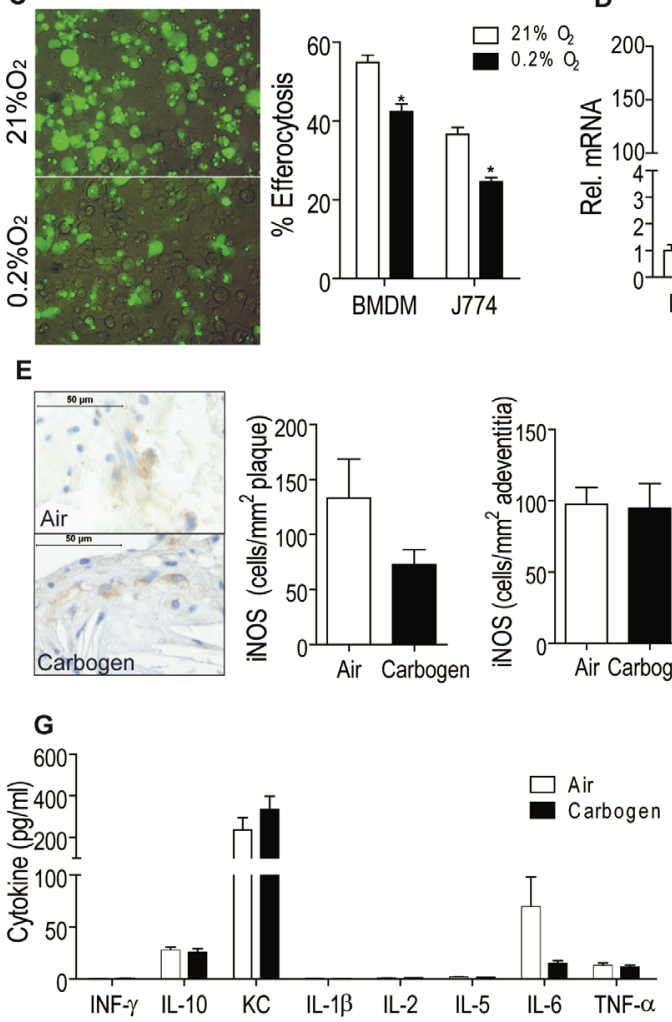

D

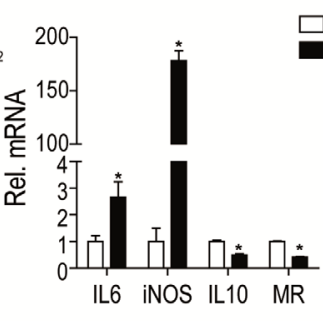

$\mathrm{F}$
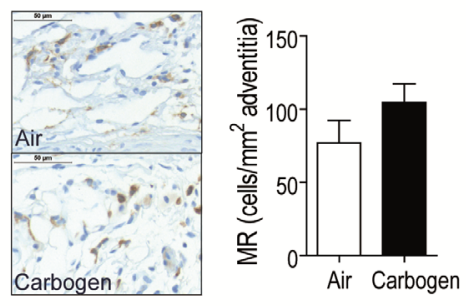

H

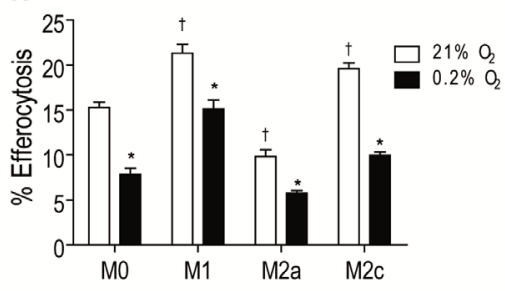

Figure 4. Neither ROS formation, nor macrophage polarization explain hypoxic repression of efferocytosis

(A) Representative images and quantifications of ROS-mediated DNA damage (8OH-dG) in aortic roots of carbogen or air-treated mice ( $n=15 /$ group, left panel), $\mathrm{LDLr}^{-/}$plasma anti-oxidant capacity relative to the standard trolox, a water-soluble vitamin $\mathrm{E}$ analogue (trolox equivalents, middle graph), and DCFDA incorporation in BMDM exposed to $21 \%, 0.2 \% \mathrm{O}_{2}$ (right graph) for 24hours. (B) Representative images and quantifications of percentage of annexin $\mathrm{A} 5$ (red) apoptotic BMDM exposed to $21 \% \mathrm{O}_{2}, 0.2 \% \mathrm{O}_{2}$ or UV-irradiated (left graph). 
Quantification of apoptosis (\% TUNEL) in 7 ketocholesterol and tunicamycin treated BMDM in $21 \% \mathrm{O}_{2}$ or $0.2 \% \mathrm{O}_{2}$. ${ }^{*}$ p-value $<0.05$ vs. normoxia, + p-value $<0.05$ vs. normoxia vehicle. (C) Representative images of calcein-AM labeled, apoptotic Jurkat $\mathrm{T}$ cells (green fluorescent) ingested by BMDM (phase contrast overlay with fluorescence) in $21 \% \mathrm{O}_{2}$ and $0.2 \% \mathrm{O}_{2}$. Quantification shows percentage of calcein+ BMDM or J774 macrophages having ingested labeled, apoptotic Jurkat T cells. *p-value<0.05. (D) M1 (IL6 - interleukin 6; iNOS - inducible nitric oxide synthase) and M2 (IL10; MR - mannose receptor) gene expression of BMDM exposed to $21 \%$ or $0.2 \% \mathrm{O}_{2}$. ${ }^{*} p$-value $<0.05$ (E) iNOS in plaque and adventitia and (F) MR expression in the adventitia of aortic roots after chronic carbogen compared to air. (G) Plasma cytokine expression in carbogen and air-treated mice $(\mathbf{H})$ Efferocytosis of apoptotic Jurkats by normoxic or hypoxic unpolarized (M0) BMDM or polarized towards an M1, M2a or M2c phenotype. * $p$-value<0.05 vs. normoxia of same polarization state, $\dagger p$-value $<0.05$ vs. normoxic M0.

\section{Hypoxia limits efferocytosis via MerTK}

Efferocytosis is a receptor-mediated process. Therefore, we studied whether hypoxia affects expression of known efferocytosis receptors. Indeed, expression of the efferocytosis receptor Mer tyrosine kinase (MerTK) and Cluster of Differentiation 36 (CD36) was reduced in hypoxic BMDM, whereas that of Scavenger receptor A (SRA) was unchanged. In contrast, low density lipoprotein receptor-related protein 1 (LRP1) was upregulated (Figure 5A). As efferocytosis capacity was decreased upon hypoxia and LRP-1 was previously not involved in efferocytosis (30), LRP1 is likely not involved.

Compromised function of MerTK was seen to underlie the impaired efferocytosis during murine atherogenesis in vivo $(17,24)$. As expected, disrupted MerTK signaling in MerTK kinase domain deficient (MerTK ${ }^{\mathrm{kd}}$ ) BMDM led to repressed efferocytosis in normoxia (Figure 5B). Importantly, while hypoxia significantly reduced the efferocytosis capacity of WT BMDM, hypoxia was not able to repress efferocytosis in MerTK ${ }^{\mathrm{kd}}$ BMDM any further (Figure 5B). This effect was specifically mediated by the MerTK ${ }^{\mathrm{kd}}$, since expression of other efferocytosis receptor expression in MerTK ${ }^{\mathrm{kd}}$ BMDM was unaltered, except for a reduction in CD36 mRNA (Figure 5C). However, as CD36 knockout did not affect efferocytosis (Figure 5D), as reported previously (30), this suggests that hypoxic repression of efferocytosis in WT BMDM is no longer effective in MerTK ${ }^{k d}$ BMDM. Hypoxia-dependent downregulation of MerTK in WT is thus likely responsible for repression of efferocytosis in a hypoxic milieu, such as the atherosclerotic plaque.
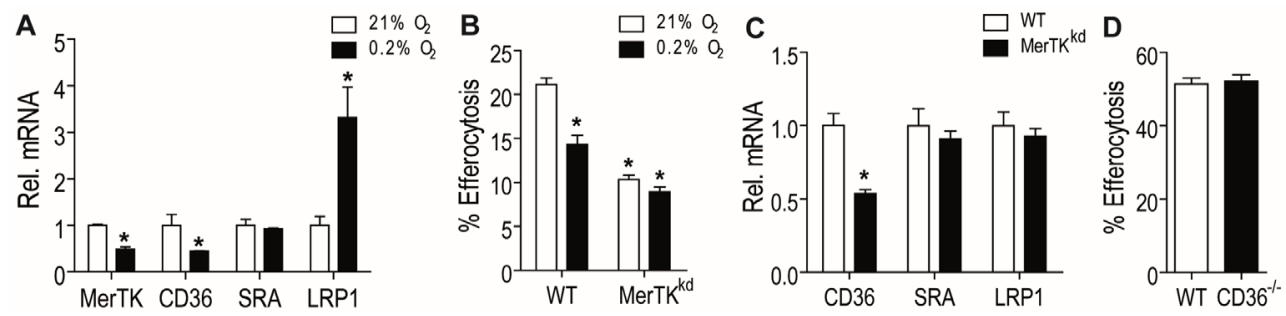

Figure 5. Hypoxia limits efferocytosis via MerTK

(A) Efferocytosis receptor gene expression (SRA - Scavenger receptor A; LRP1 - Low density lipoprotein receptorrelated protein 1 ) in normoxia or $0.2 \% \mathrm{O}_{2} .{ }^{*}$ p-value $<0.05$ vs. normoxia of same gene. (B) Efferocytosis in wildtype and MerTK $^{\mathrm{kd}}$ BMDM exposed to $21 \%$ or $0.2 \% \mathrm{O}_{2}$. ${ }^{*}$ p-value $<0.05$ vs. wildtype normoxia. (C) Efferocytosis receptor gene expression in wt and MerTK ${ }^{\mathrm{kd}}$ BMDMs. ${ }^{*} \mathrm{p}$-value<0.05 vs. wt of same gene. (D) Efferocytosis of apoptotic Jurkats by wt and $\mathrm{CD} 36^{-1-} \mathrm{BMDM}$. 


\section{DISCUSSION}

This study revealed that reversal of plaque hypoxia can inhibit the progression of atherosclerotic plaques to a vulnerable phenotype, by reducing the development of the plaque-destabilizing necrotic core. Also, it was shown that murine plaque macrophages are hypoxic from the initiation of atherosclerosis on, using the hypoxia-specific marker pimonidazole. Importantly, we revealed that hyperoxic carbogen gas not only achieved reoxygenation of atherosclerotic plaques, liver and spleen in $\mathrm{LDLr}^{-/}$mice, but also prevented apoptotic cell accumulation in the plaque.

Apoptosis induction in vitro was not augmented by hypoxia, suggesting that reoxygenation restored the known deficiency in apoptotic cell clearance in atherosclerosis (23). Mechanistically, hypoxia-dependent transcriptional downregulation of MerTK, and subsequent reduction of apoptotic cell clearance by MerTK is the likely underlying cause for hampered efferocytosis by hypoxic macrophages. Thus, this study is the first to show a causal role of hypoxia in plaque destabilization, specifically in efferocytosis.

In addition, plaque thrombogenicity was studied using TF expression, a key trigger of the extrinsic coagulation system causally linked to plaque thrombosis (28). Interestingly, hypoxia enhanced macrophage expression of TF, supporting the prior correlation of human plaque hypoxia to intraplaque hemorrhage (6). Nevertheless, carbogen did not prevent the hypoxia-induced expression of plaque TF.

The oxygen debt in atherosclerosis is thought to arise from the sizeable oxygen-consuming metabolism of inflammatory cells, as even macrophages of $\mathrm{LDLr}^{-/}$fatty streaks directly adjacent to arterial oxygen supply, within the 100-200 $\mu$ m oxygen diffusion distance, were hypoxic. This was already suggested by our previous work, showing that human plaque macrophages were hypoxic, despite the close proximity - within $40 \mu \mathrm{m}$ - of arterial blood oxygen supply (6). Moreover, hypoxia was also detected in most tissue-resident leukocytes of lymphoid organs in $\mathrm{LDLr}^{-/-}$mice. Not surprisingly, as hypoxia was found in many, if not all tissues with an extensive inflammatory influx, such as wound healing, obesity, rheumatoid arthritis, and tumors (31-34). As pimonidazole only forms adducts in low oxygen concentrations $(<10 \mathrm{mmHg}$ oxygen (21)), this indicates that hypoxia in plaque macrophage arises from a high oxygen demand exceeding oxygen supply. Indeed, activated macrophages, in need of ATP for protein production and migration, show 9-fold enhancement of their oxygen consumption (35). Oxygen usage of activated macrophages would then compare to the notoriously high $\mathrm{O}_{2}$ consumption by cardiac myocytes (36) which were indeed also positive for pimonidazole and sensitive to carbogen-mediated reoxygenation (data not shown). Therefore, it is highly likely that upon activation, macrophages rapidly become hypoxic, even within the oxygen-diffusion limit.

Once hypoxic, macrophages will primarily rely on glycolysis as main energy supply, as suggested by a hypoxia-dependent increase in glucose uptake (37). Together, this may explain prior results of plaques with low glucose and ATP content, and high lactate levels (38). Increased glycolytic activity has been linked with M1 polarization of macrophages (39). Indeed, hypoxic macrophages presented with a pro-inflammatory M1 phenotype in vitro, although reoxygenation in vivo failed to switch polarization. Nevertheless, tumorassociated macrophages located in hypoxic tumor regions, express an M2-like profile, and both $\mathrm{M} 1$ and $\mathrm{M} 2$ marker expression was found in hypoxic adipose tissue macrophages 
$(40,41)$. Hence, macrophage subtype distinction might not be as clear in vivo, and despite hypoxia, the microenvironment seems to influence macrophage polarization/function also in the plaque. M2c macrophages are the most superior efferocytic macrophage subtype in humans. (29). However, neither M1 nor M2 polarization could protect from hypoxiamediated reduction in efferocytosis. Mechanistically, low oxygen tension downregulated MerTK and CD36 expression, while upregulating LRP1. Both CD36 and LRP were not involved in efferocytosis in vitro as shown by us and others (30). Hypoxic upregulation of LRP1 expression may be efferocytosis-independent, as LRP1 has a myriad of other functions related to atherosclerosis (42). Only MerTK ${ }^{k d}$ mimicked the reduced efferocytosis seen in hypoxic macrophages. In fact, MerTK ${ }^{k d}$ has been shown to aggravate atherosclerosis in mice and worsen lupus-like autoimmunity $(17,24,43)$, suggesting a role of MerTK in chronic inflammatory diseases.

While MerTK ${ }^{\mathrm{kd}}$ bone marrow accelerated both atherosclerotic plaque size, as well as necrotic core size (17), carbogen treatment only affected the latter. This may be explained by the potentially partial inhibition of MerTK expression, the intermittent nature of the carbogen treatment and treatment onset after initiation of atherosclerosis. While MerTK ${ }^{\mathrm{kd}}$ transplanted $\mathrm{LDLr}^{-/-}$recipient mice presented with a continuous and complete absence of MerTK activity prior to plaque initiation (17), normoxic macrophages in vitro show a $50 \%$ increase in MerTK expression compared to hypoxic macrophages. Treatment of MerTK ${ }^{\mathrm{kd}}$ mice with carbogen gas could provide definite proof of the MerTK-mediated carbogen effect.

The current study is limited by the systemic nature of carbogen exposure. Therefore, many aspects of peripheral processes instrumental in atherogenesis were examined. Carbogen did not affect erythropoiesis, myelopoiesis, thrombogenicity or plasma cholesterol level. Also, blood pressure and heart rate were unaffected $(44,45)$. Although unlikely, we cannot fully exclude an indirect effect of carbogen on atherogenesis.

Our results unveil an exciting new process to exploit for therapeutic strategies for atherosclerosis. In mice, carbogen gas was able to stabilize the atherosclerotic plaque; if translatable to the human situation, this may potentially prevent plaque ruptures and thrombosis. Indeed, carbogen and hyperbaric oxygen therapy were safely and successfully applied in cancer and diabetic foot patients, respectively $(46,47)$. While in humans with sleep apnea, maintained oxygen supply through continuous positive airway pressure therapy already proved effective in reversing sub-clinical atherosclerosis (48), effectiveness in existing human atherosclerosis remains unclear. Additionally, currently developed tools for imaging of hypoxia $(13,49)$ can be used to monitor the efficacy of carbogen to alter human plaque hypoxia and stability.

In conclusion, carbogen-mediated reoxygenation supports the role of plaque hypoxia as a driver of plaque instability through dysfunctional MerTK-mediated clearance of apoptotic cells and subsequent necrotic core expansion. Nevertheless, translation of carbogen therapy to human atherosclerosis will need to be confirmed using non-invasive imaging of plaque hypoxia and stability. 


\section{ACKNOWLEDGMENTS}

Research was supported by a VENI fellowship of the Netherlands Organization of Scientific research (to J.C.S. 016.116.017), a PhD-student fellowship from the Cardiovascular Research Institute Maastricht (to T.T.), a Dr. E. Dekker grant from the Dutch Heart Foundation (M.M.D. 2007 T034 and 2012T079) NIH grant HL084312 (to E.A.F.). 


\section{REFERENCES}

1. Carmeliet P. Angiogenesis in life, disease and medicine. Nature. 2005 Dec 15;438(7070):932-6.

2. Fong C-C, Zhang Q, Shi Y-F, Wu RSS, Fong W-F, Yang M. Effect of hypoxia on RAW264.7 macrophages apoptosis and signaling. Toxicology. 2007 Jun 3;235(1-2):52-61.

3. Hansson GK. Inflammation, atherosclerosis, and coronary artery disease. N Engl J Med. 2005 Apr 21;352(16):1685-95.

4. Tabas I. Macrophage death and defective inflammation resolution in atherosclerosis. Nat Rev Immunol. 2010 Jan;10(1):36-46.

5. Virmani R, Burke AP, Farb A, Kolodgie FD. Pathology of the unstable plaque. Prog Cardiovasc Dis. 2002 Mar;44(5):349-56.

6. Sluimer JC, Gasc J-M, van Wanroij JL, Kisters N, Groeneweg M, Sollewijn Gelpke MD, et al. Hypoxia, hypoxia-inducible transcription factor, and macrophages in human atherosclerotic plaques are correlated with intraplaque angiogenesis. J Am Coll Cardiol. 2008 Apr 1;51(13):1258-65.

7. Lever E, Sheer D. The role of nuclear organization in cancer. J Pathol. 2010 Jan;220(2):114-25.

8. Björnheden T, Levin M, Evaldsson M, Wiklund O. Evidence of hypoxic areas within the arterial wall in vivo. Arterioscler Thromb Vasc Biol. 1999 Apr;19(4):870-6.

9. Ramkhelawon B, Yang Y, van Gils JM, Hewing B, Rayner KJ, Parathath S, et al. Hypoxia induces netrin-1 and Unc5b in atherosclerotic plaques: mechanism for macrophage retention and survival. Arterioscler Thromb Vasc Biol. 2013 Jun;33(6):1180-8.

10. Parathath S, Mick SL, Feig JE, Joaquin V, Grauer L, Habiel DM, et al. Hypoxia is present in murine atherosclerotic plaques and has multiple adverse effects on macrophage lipid metabolism. Circ Res. 2011 Oct 28;109(10):1141-52.

11. Murdoch C, Muthana M, Lewis CE. Hypoxia regulates macrophage functions in inflammation. J Immunol. 2005 Nov 15;175(10):6257-63.

12. Burke B, Giannoudis A, Corke KP, Gill D, Wells M, Ziegler-Heitbrock L, et al. Hypoxia-induced gene expression in human macrophages: implications for ischemic tissues and hypoxia-regulated gene therapy. Am J Pathol. 2003 Oct;163(4):1233-43.

13. Dubois LJ, Lieuwes NG, Janssen MHM, Peeters WJM, Windhorst AD, Walsh JC, et al. Preclinical evaluation and validation of [18F]HX4, a promising hypoxia marker for PET imaging. Proc Natl Acad Sci U S A. 2011/08/30 ed. 2011 Aug 30;108(35):14620-5.

14. Jordan BF, Magat J, Colliez F, Ozel E, Fruytier A-C, Marchand V, et al. Mapping of oxygen by imaging lipids relaxation enhancement: a potential sensitive endogenous MRI contrast to map variations in tissue oxygenation. Magn Reson Med. 2012/10/02 ed. 2013 Sep;70(3):732-44.

15. van Laarhoven HWM, Bussink J, Lok J, Verhagen I, Punt CJA, Heerschap A, et al. Modulation of hypoxia in murine liver metastases of colon carcinoma by nicotinamide and carbogen. Radiat Res. 2005/09/03 ed. 2005 Sep;164(3):245-9.

16. Kaanders JH a M, Bussink J, van der Kogel AJ. ARCON: a novel biology-based approach in radiotherapy. Lancet Oncol. 2002/12/11 ed. 2002 Dec;3(12):728-37.

17. Ait-Oufella H, Pouresmail V, Simon T, Blanc-Brude O, Kinugawa K, Merval R, et al. Defective mer receptor tyrosine kinase signaling in bone marrow cells promotes apoptotic cell accumulation and accelerates atherosclerosis. Arterioscler Thromb Vasc Biol. 2008 Aug;28(8):1429-31. 
18. Scott RS, McMahon EJ, Pop SM, Reap E a, Caricchio R, Cohen PL, et al. Phagocytosis and clearance of apoptotic cells is mediated by MER. Nature. 2001/05/11 ed. 2001 May 10;411(6834):207-11.

19. Febbraio M, Abumrad N a., Hajjar DP, Sharma K, Cheng W, Pearce SF, et al. A null mutation in murine CD36 reveals an important role in fatty acid and lipoprotein metabolism. J Biol Chem. 1999 Jul 2;274(27):19055-62.

20. Janssen B, Debets J, Leenders P, Smits J. Chronic measurement of cardiac output in conscious mice. Am J Physiol Regul Integr Comp Physiol. 2002/02/08 ed. 2002 Mar;282(3):R928-35.

21. Chou S-C, Azuma Y, Varia MA, Raleigh JA. Evidence that involucrin, a marker for differentiation, is oxygen regulated in human squamous cell carcinomas. $\mathrm{Br} J$ Cancer. 2004/02/05 ed. 2004 Feb 9;90(3):728-35.

22. Kleiter MM, Thrall DE, Malarkey DE, Ji X, Lee DYW, Chou S-C, et al. A comparison of oral and intravenous pimonidazole in canine tumors using intravenous $\mathrm{CCl}-103 \mathrm{~F}$ as a control hypoxia marker. Int J Radiat Oncol Biol Phys. 2006 Feb 1;64(2):592-602.

23. Schrijvers DM, De Meyer GRY, Kockx MM, Herman AG, Martinet W. Phagocytosis of apoptotic cells by macrophages is impaired in atherosclerosis. Arterioscler Thromb Vasc Biol. 2005 Jun;25(6):1256-61.

24. Thorp E, Cui D, Schrijvers DM, Kuriakose G, Tabas I. Mertk receptor mutation reduces efferocytosis efficiency and promotes apoptotic cell accumulation and plaque necrosis in atherosclerotic lesions of apoe-/- mice. Arterioscler Thromb Vasc Biol. 2008 Aug;28(8):1421-8.

25. Fischer MAJG, Gransier TJM, Beckers LMG, Bekers O, Bast A, Haenen GRMM. Determination of the antioxidant capacity in blood. Clin Chem Lab Med. 2005/10/07 ed. 2005 Jan 1;43(7):735-40.

26. Sluimer JC, Kisters N, Cleutjens KB, Volger OL, Horrevoets AJ, van den Akker LH, et al. Dead or alive: gene expression profiles of advanced atherosclerotic plaques from autopsy and surgery. Physiol Genomics. 2007 Aug 20;30(3):335-41.

27. Seijkens T, Hoeksema M a, Beckers L, Smeets E, Meiler S, Levels J, et al. Hypercholesterolemia-induced priming of hematopoietic stem and progenitor cells aggravates atherosclerosis. FASEB J. 2014 May;28(5):2202-13.

28. Borissoff JI, Heeneman S, Kilinç E, Kassák P, Van Oerle R, Winckers K, et al. Early atherosclerosis exhibits an enhanced procoagulant state. Circulation. 2010 Aug 24;122(8):821-30.

29. Zizzo G, Hilliard BA, Monestier M, Cohen PL. Efficient clearance of early apoptotic cells by human macrophages requires M2c polarization and MerTK induction. J Immunol. 2012 Oct 1;189(7):3508-20.

30. Li Y, Gerbod-Giannone M-C, Seitz H, Cui D, Thorp E, Tall AR, et al. Cholesterol-induced apoptotic macrophages elicit an inflammatory response in phagocytes, which is partially attenuated by the Mer receptor. J Biol Chem. 2006 Mar 10;281(10):6707-17.

31. Kennedy a S, Raleigh J a, Perez GM, Calkins DP, Thrall DE, Novotny DB, et al. Proliferation and hypoxia in human squamous cell carcinoma of the cervix: first report of combined immunohistochemical assays. Int J Radiat Oncol Biol Phys. 1997 Mar 1;37(4):897-905.

32. Lee Y-A, Kim JY, Hong S-J, Lee S-H, Yoo MC, Kim KS, et al. Synovial proliferation differentially affects hypoxia in the joint cavities of rheumatoid arthritis and osteoarthritis patients. Clin Rheumatol. 2007 Dec;26(12):2023-9.

33. Haroon ZA, Raleigh JA, Greenberg CS, Dewhirst MW. Early wound healing exhibits cytokine surge without evidence of hypoxia. Ann Surg. 2000 Jan;231(1):137-47.

34. Rausch ME, Weisberg S, Vardhana P, Tortoriello D V. Obesity in C57BL/6J mice is characterized by adipose tissue hypoxia and cytotoxic T-cell infiltration. Int J Obes (Lond). 2008 Mar;32(3):451-63. 
35. Rist RJ, Naftalin RJ. Glucose- and phorbol myristate acetate-stimulated oxygen consumption and superoxide production in rat peritoneal macrophages is inhibited by dexamethasone. Biochem $\mathrm{J}$. 1993/04/15 ed. 1993 Apr 15;291 ( Pt 2:509-14.

36. Wagner BA, Venkataraman S, Buettner GR. The rate of oxygen utilization by cells. Free Radic Biol Med. 2011/06/15 ed. 2011 Aug 1;51(3):700-12.

37. Folco EJ, Sheikine Y, Rocha VZ, Christen T, Shvartz E, Sukhova GK, et al. Hypoxia but not inflammation augments glucose uptake in human macrophages: Implications for imaging atherosclerosis with 18fluorine-labeled 2-deoxy-D-glucose positron emission tomography. J Am Coll Cardiol. 2011 Aug 2;58(6):603-14.

38. Levin M, Leppänen O, Evaldsson M, Wiklund O, Bondjers G, Björnheden T. Mapping of ATP, glucose, glycogen, and lactate concentrations within the arterial wall. Arterioscler Thromb Vasc Biol. 2003 Oct $1 ; 23(10): 1801-7$

39. Kawaguchi T, Veech RL, Uyeda K. Regulation of energy metabolism in macrophages during hypoxia. Roles of fructose 2,6-bisphosphate and ribose 1,5-bisphosphate. J Biol Chem. 2001 Jul 27;276(30):28554-61

40. Fujisaka S, Usui I, Ikutani M, Aminuddin A, Takikawa A, Tsuneyama K, et al. Adipose tissue hypoxia induces inflammatory M1 polarity of macrophages in an HIF-1 $\alpha$-dependent and HIF-1 $\alpha$-independent manner in obese mice. Diabetologia. 2013 Jun;56(6):1403-12.

41. Van Overmeire E, Laoui D, Keirsse J, Van Ginderachter JA. Hypoxia and tumor-associated macrophages: A deadly alliance in support of tumor progression. Oncoimmunology. 2014 Jan 1;3(1):e27561.

42. Lillis AP, Van Duyn LB, Murphy-Ullrich JE, Strickland DK. LDL receptor-related protein 1: unique tissuespecific functions revealed by selective gene knockout studies. Physiol Rev. 2008 Jul;88(3):887-918.

43. Cohen PL, Caricchio R, Abraham V, Camenisch TD, Jennette JC, Roubey RAS, et al. Delayed apoptotic cell clearance and lupus-like autoimmunity in mice lacking the c-mer membrane tyrosine kinase. J Exp Med. 2002 Jul 1;196(1):135-40.

44. Brizel DM, Lin S, Johnson JL, Brooks J, Dewhirst MW, Piantadosi CA. The mechanisms by which hyperbaric oxygen and carbogen improve tumour oxygenation. Br J Cancer. 1995 Nov;72(5):1120-4.

45. Kergoat H, Faucher C. Effects of oxygen and carbogen breathing on choroidal hemodynamics in humans. Invest Ophthalmol Vis Sci. 1999/11/05 ed. 1999 Nov;40(12):2906-11.

46. Hoskin P, Rojas A, Saunders M. Accelerated radiotherapy, carbogen, and nicotinamide (ARCON) in the treatment of advanced bladder cancer: mature results of a Phase II nonrandomized study. Int J Radiat Oncol Biol Phys. 2009 Apr 1;73(5):1425-31.

47. Lipsky B a., Berendt AR. Hyperbaric oxygen therapy for diabetic foot wounds: has hope hurdled hype? Diabetes Care. 2010 May;33(5):1143-5.

48. Drager LF, Bortolotto LA, Figueiredo AC, Krieger EM, Lorenzi GF. Effects of continuous positive airway pressure on early signs of atherosclerosis in obstructive sleep apnea. Am J Respir Crit Care Med. 2007 Oct 1;176(7):706-12.

49. Silvola JMU, Saraste A, Forsback S, Laine VJO, Saukko P, Heinonen SE, et al. Detection of hypoxia by [18F]EF5 in atherosclerotic plaques in mice. Arterioscler Thromb Vasc Biol. 2011 May;31(5):1011-5. 
Chapter 2

SUPPLEMENTAL DATA

\begin{tabular}{lll}
\hline Gene & Forward primer & Reverse primer \\
\hline Cyclophylin & CAAATGCTGGACCAAACACAA & TTCACCTTCCCAAAGACCACAT \\
18S rRNA & GTAACCCGTTGAACCCCATT & CCATCCAATCGGTAGTAGCG \\
IL6 & ACAAGTCGGAGGCTTAATTACACAT & TTGCCATTGCACAACTCTTTTC \\
iNOS & CAGCTGGGCTGTACAAACCTT & CATTGCAAGTGAAGCGTTTCG \\
IL10 & TGCTCCTAGAGCTGCGGACT & TGCTCCTAGAGCTGCGGACT \\
MR & GCAAATGGAGCCGTCTGTGC & CTCGTGGATCTCCGTGACAC \\
MerTK & CACAATGACAAAGGACTGACG & AGTAGCCATCAAAACCAGGG \\
CD36 & GCCAAGCTATTGCGACATGA & AAAAGAATCTCAATGTCCGAGACTTT \\
LRP1 & GGACCACCATCGTGGAAA & TCCCAGCCACGGTGATAG \\
\hline
\end{tabular}

Supplemental table 1. Sequences of quantitative RT-PCR primers 
A

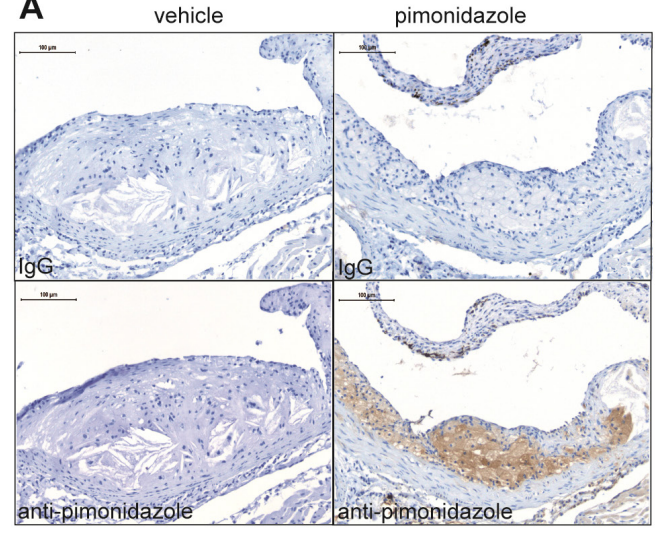

C

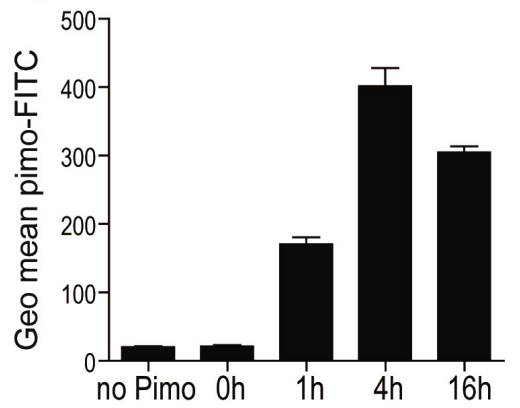

B

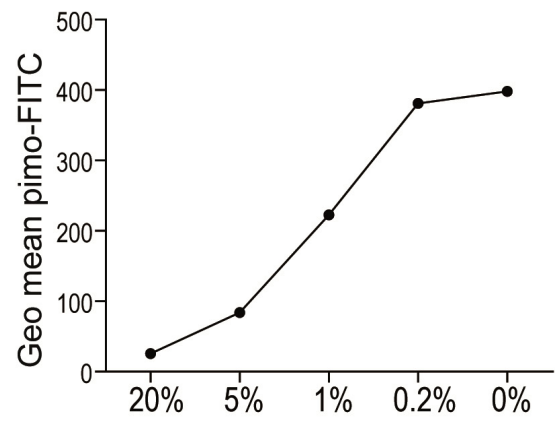

D

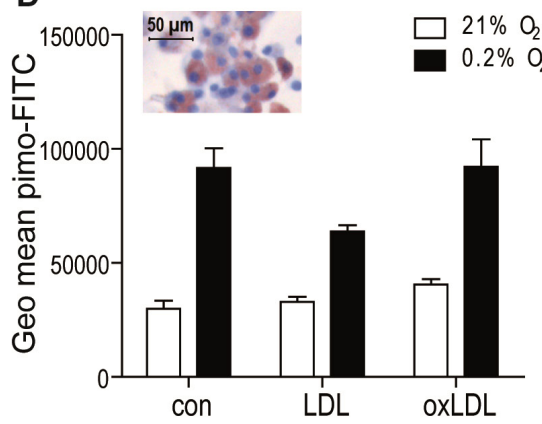

Supplemental figure 1. Specificity of pimonidazole as a hypoxic marker

(A) Representative pictures of pimonidazole detection (brown, DAB) in $\mathrm{LDLr}^{\%}$ mice receiving pimonidazole injection (right panels) or vehicle (left panels), and immunohistochemistry using anti-pimonidazole antibody (bottom panels) or isotype IgG control (top panels). (B) THP-1 macrophages exposed to decreasing oxygen concentrations show increased pimonidazole incorporation. (C) Pimonidazole positivity in THP-1 macrophages subjected to $0.2 \%$ oxygen for increasing time periods. (D) BMDMs treated with LDL, oxLDL or control only show increased pimonidazole incorporation upon exposure to $0.2 \% \mathrm{O} 2$ not after incubation in normoxia. Foam cell formation was confirmed with Oil Red O. All graphs present geo mean fluorescence of anti-pimonidazole FITC measured by flow cytometry. 
A

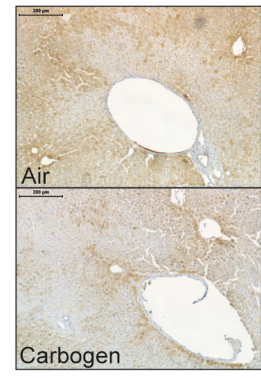

C

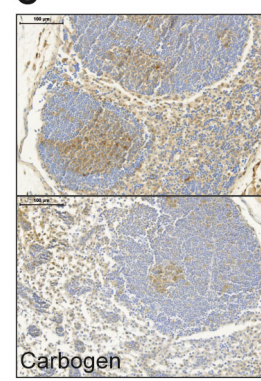

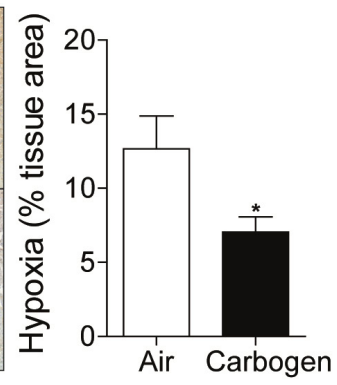

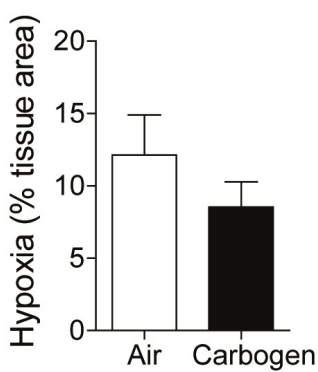

B
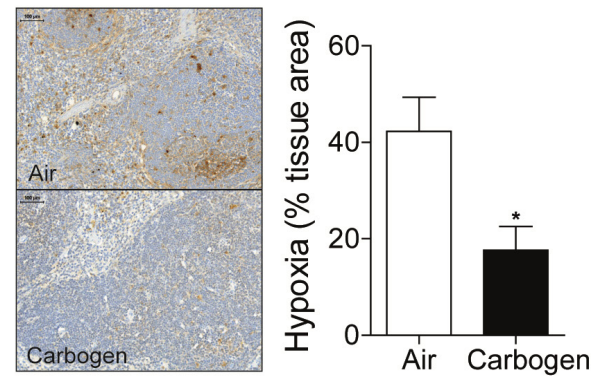

D

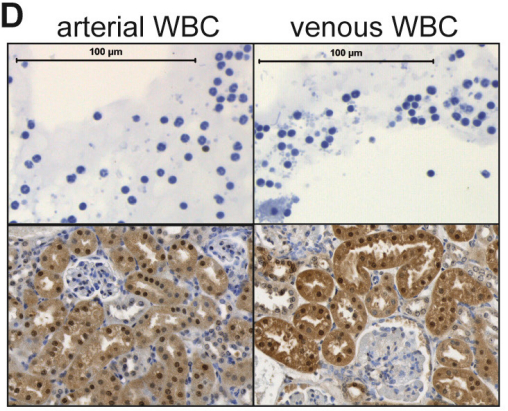

Supplemental figure 2. Carbogen reoxygenated liver spleen and lymph nodes

(A) Representative pictures and quantifications of liver, (B) spleen, and (C) lymph node stained with pimonidazole (brown) from air or carbogen treated $\mathrm{LDLr}^{-/-}$mice. ${ }^{*} \mathrm{p}$-value<0.05. D. Representative pictures of pimonidazole stained arterial and venous white blood cells of two separate $\mathrm{LDLr}^{--}$mice, with their respective mouse-matched kidney sections stained as positive control. 

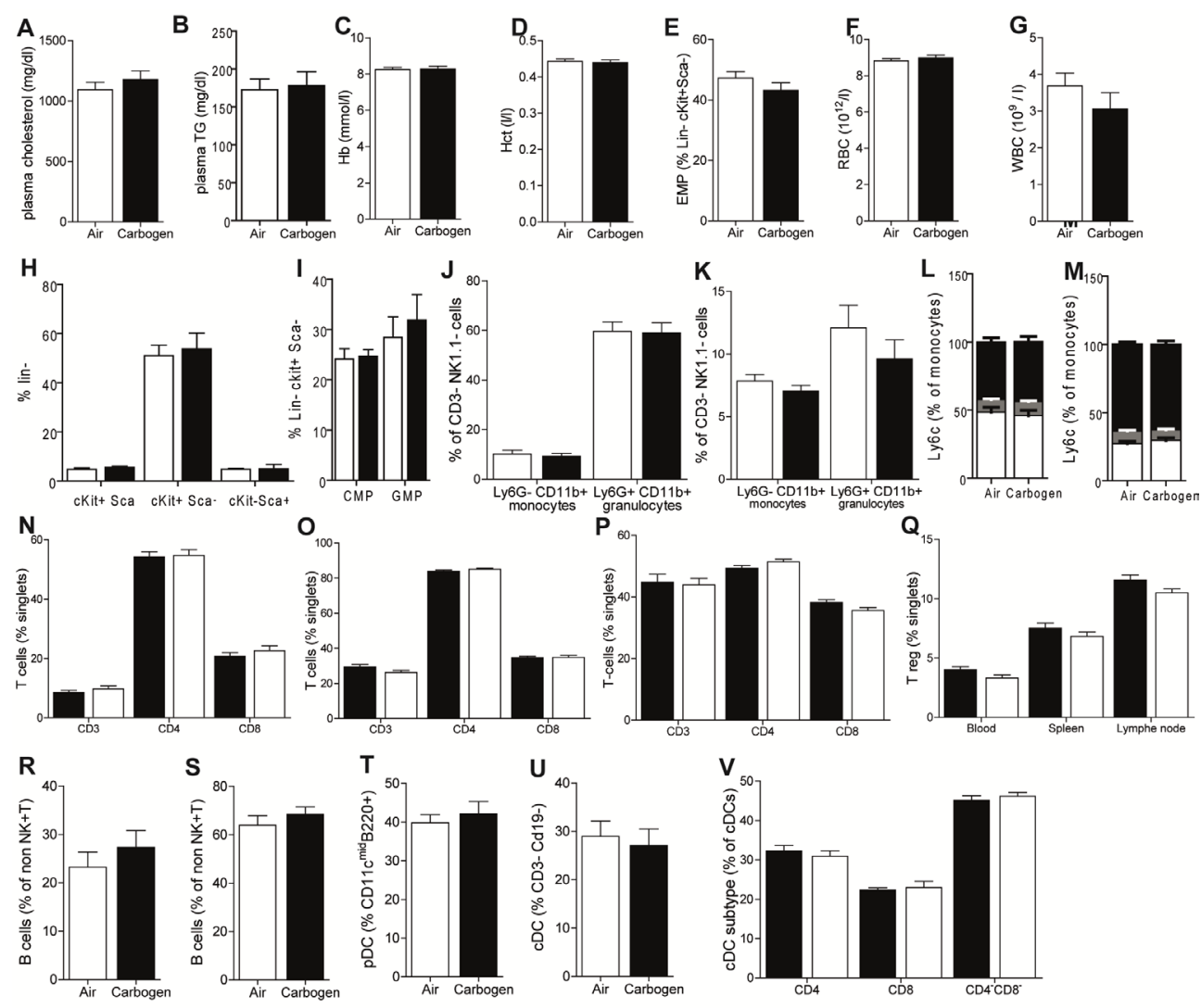

Supplemental figure 3. Cholesterol and hematopoiesis are unchanged after carbogen exposure

(A) plasma cholesterol, (B) plasma triglycerides (TG), (C) hemoglobin ( $\mathrm{Hb})$, and (D) hematocrit ( $\mathrm{Ht}),(\mathrm{E})$ bone marrow erythrocyte-megakaryocyte progenitors (EMP, CD16/32-CD34-), (F) absolute erythrocyte counts and (G) absolute white blood cell counts were similar between carbogen (black bars) or air-treated mice (white bars). (H) Bone marrow stem cells, (I) common myeloid progenitor (CMP), and granulocyte-macrophage progenitor (GMP) were similar between carbogen or air-treated mice. $(\mathrm{J})$ Blood and $(\mathbf{K})$ spleen monocyte and granulocyte populations and (L) blood and (M) spleen Ly6C monocytic subsets (Ly6Chigh, white bar; Ly6Clow, grey bar; Ly6Cneg, black bar) were similar between carbogen or air-treated mice N. Total T cells (CD3), Thelper (CD4) and cytotoxic T cells (CD8) were similar between carbogen or air-treated mice in blood, (O) spleen, and (P) lymph nodes. (Q) T regulatory cell (T-reg) counts were also similar in blood, spleen and lymph nodes of carbogen or airtreated mice. (R) B-cells were similar between carbogen or air-treated mice in blood, and (S) spleen. (T) Plasmacytoid dendritic cells $(\mathrm{pDC}),(\mathbf{U})$ conventional $\mathrm{DC}(\mathrm{cDC})$ and $(\mathbf{V}) \mathrm{cDC}$ subsets were similar between carbogen or air-treated mice in spleen. 


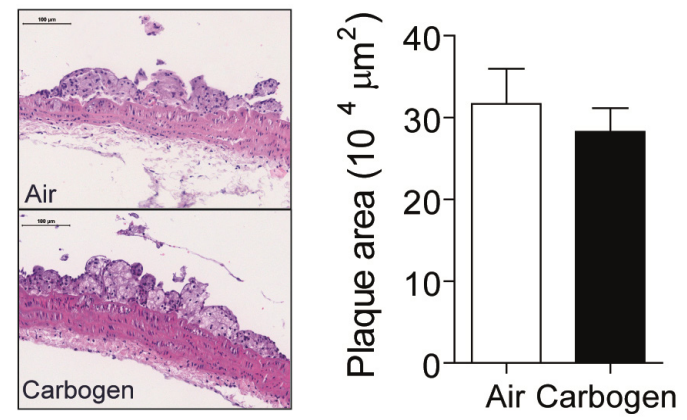

Supplemental figure 4. Carbogen did not change plaque initiation in the aortic arch

Representative hematoxylin and eosin images and quantification of aortic arch plaques of LDLr ${ }^{-/}$mice exposed to an initial 4 weeks of WTD followed by 4 weeks of diet in combination with air (white bars) or carbogen gas (black bars). 


\section{Chapter 3}

\section{Myeloid prolyl hydroxylase domain-containing protein $\mathbf{2}$ deficiency leads to larger, but more stable atherosclerotic plaques in mice}

Theelen TL, Marsch E, Demandt JA, Fallais S, Tullemans BM, van der Vorst EP, Donners MM, Gijbels MJ, Welting TJ, Reutelingsperger CP, Mastenbroek TG, Cosemans JM, Willems

BA, Schurgers L, Carmeliet P, Biessen EA, Daemen MJ, Sluimer JC

Submitted

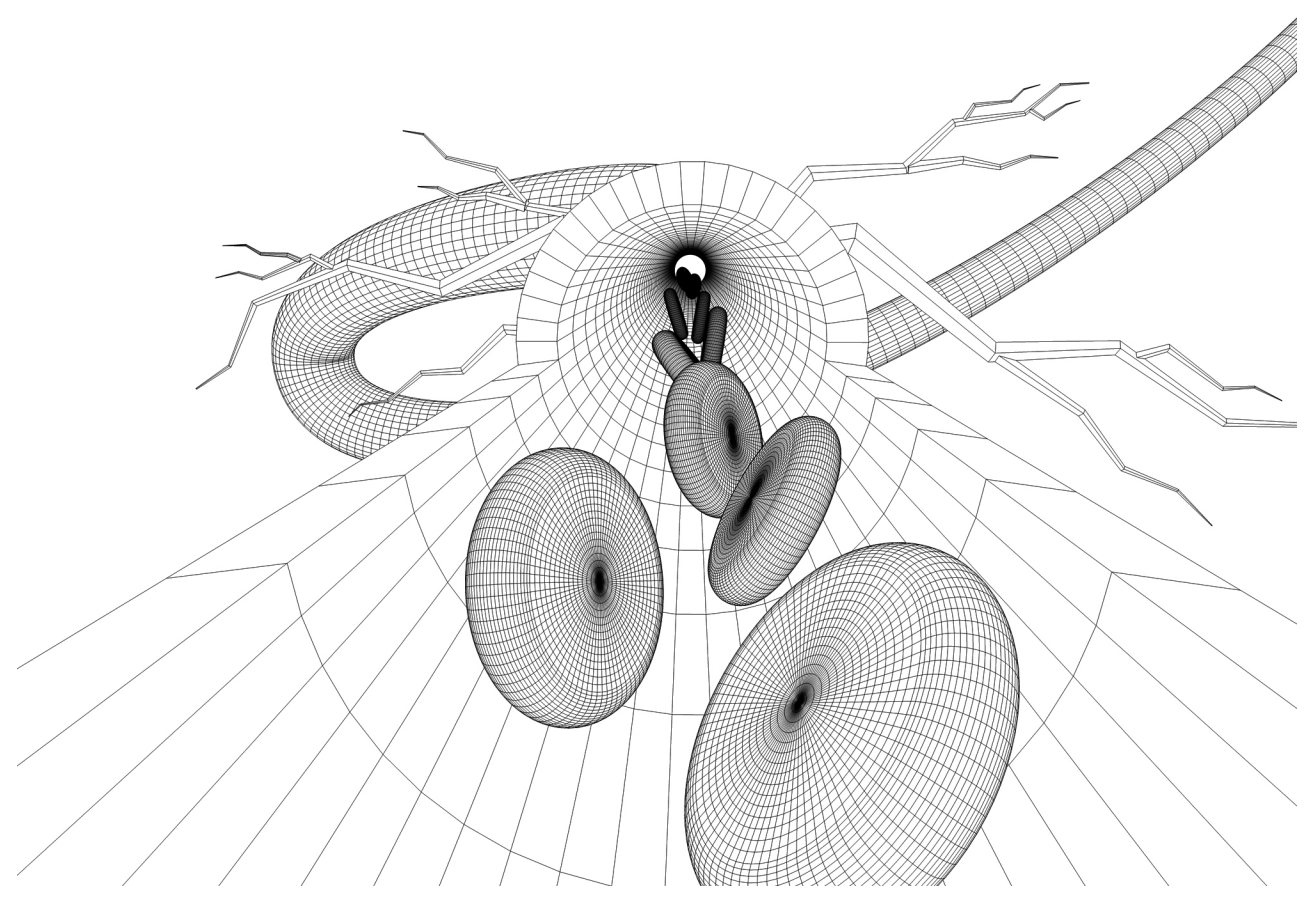




\section{ABSTRACT}

\section{Objective}

Angiogenesis and macrophage hypoxia in atherosclerotic plaques are causally related to plaque inflammation and progression, but the underlying triggers remain unknown. Hypoxia-dependent, pro-angiogenic signaling is mainly regulated by the oxygen sensor prolyl hydroxylase domain protein 2 (PHD2), mediated by its stabilization of the hypoxiainducible transcript factor (HIF) $1 \alpha$. Here, the effect of myeloid-specific PHD2 knockout was studied in atherosclerotic mice.

\section{Approach and results}

PHD2 expression was analyzed in human atherosclerotic plaques by immunohistochemistry and microarray. We found that PHD2 expression correlated mainly with macrophages. To further study PHD2 knockout in atherosclerosis, low-density lipoprotein receptor knockout $\left(\mathrm{LDLr}^{-/}\right)$mice were crossed with LysMCre ${ }^{+/-}$PHD2 floxed (PHD2 cKO) mice. The resulting PHD2 cKO mice were put on a high cholesterol diet for either 6 or 12 weeks. The aortic root and brachiocephalic artery were excised, paraffin embedded and sequentially sectioned for atherosclerosis characterization.

Myeloid PHD2 deficiency resulted in significantly larger atherosclerotic plaques in the aortic root and brachiocephalic artery in all plaque stages (early $+60 \%$, intermediate $+50 \%$, advanced $+40 \%$ ). Plaque and adventitial angiogenesis were similar, while PHD2 cKO resulted in a remarkable pro-fibrotic phenotype of atherosclerotic plaques accounting for the larger plaques of PHD2 cKO mice (early $+65 \%$; intermediate $+70 \%$, advanced $+22 \%$ collagen). This collagen expansion stabilized atheromas, as cap thickness was increased in intermediate $(+47 \%)$ and advanced $(+29 \%)$ plaques.

Macrophages generally regulate collagen content by matrix metalloproteinase (MMP)dependent collagen degradation. PHD2 CKO macrophage MMP activity was unchanged and plaque macrophage content decreased by PHD2 cKO. This suggests a net reduction in collagen degradation. Also, PHD2 cKO macrophages in vitro increased collagen synthesis (Sirius red $+13 \%, p<0.01$ ). In addition, SMC content increased slightly during plaque initiation, although SMC collagen synthesis, proliferation and migration was not altered by PHD2 cKO macrophage conditioned medium. Nevertheless, the ratio between collagenproducing SMC and collagen-degrading macrophages was increased. Thus, the increased ratio between smooth muscle cell and macrophage content, together with the increase in macrophage collagen production, most likely accounts for the net increase in plaque collagen.

\section{Conclusions}

Myeloid PHD2 deficiency shifts the balance between collagen production and degradation to net collagen accumulation, thus firmly enhancing plaque stability. 


\section{INTRODUCTION}

Ruptured atherosclerotic plaques account for the highest number of deaths in the Western world through their devastating consequences such as ischemic heart disease (1). Several hallmarks have been identified in the transition from a clinically silent, stable atherosclerotic plaque to a clinically relevant, unstable atherosclerotic plaque: inflammation, intra-plaque hemorrhage, thinning of the fibrous cap, a large necrotic core, hypoxia and angiogenesis (2). Although plaque angiogenesis is a well-known risk factor in humans, the underlying mechanisms and its exact contribution to plaque rupture remain unknown, as murine models of spontaneous plaque angiogenesis are scarce.

Human plaque angiogenesis is thought to be triggered by hypoxia (3). Even fatty streaks are hypoxic, suggesting that increased oxygen consumption on top of limited oxygen diffusion determines plaque hypoxia (4). We and others have previously demonstrated that macrophages, the most prominent cell type in atherosclerotic lesions, are the main hypoxic cell type present (5). It has been shown that hypoxia stimulates macrophage expression of several pro-angiogenic factors such as vascular endothelial growth factor (6), thereby linking hypoxia signaling to plaque angiogenesis. In addition, hypoxia triggers inflammation and lipid accumulation, both factors worsening atherosclerosis. Hypoxia related gene expression is mainly coordinated by the transcription factor hypoxia inducible factor (HIF) 1 alpha, via its binding to the hypoxia responsive element within their promotor region. In the presence of oxygen, HIF1 $\alpha$ is marked for degradation by oxygen-dependent hydroxylation by prolyl-hydroxylase domain enzymes (PHD), predominantly by PHD2. In the absence of its essential co-factor oxygen, PHDs are inactive, preventing HIF degradation. HIF levels are thereby tightly regulated, and PHD2 represents the main regulatory switch for the cellular hypoxia response (7).

It has been demonstrated that knockdown of the master regulator PHD2 in macrophages increased angiogenesis under acute, ischemic conditions through HIF-independent signaling via nuclear factor kappa B (NFKB) (8). In addition, PHD2 knockdown evoked an M2-like macrophage phenotype, which is considered pro-angiogenic, but antiinflammatory and involved in pro-fibrotic wound healing processes (8).

As multiple processes are affected by macrophage PHD2, and often have opposing effects on plaque stability, we investigated the consequences of PHD2 knockout in macrophages on atherogenesis and plaque angiogenesis using lysosyme M-driven Cre (LysMCre) PHD2 floxed $\left(\mathrm{PHD}^{\mathrm{fl} / \mathrm{fl}}\right.$ ) mice on a low density lipoprotein receptor (LDLr) knock out background. 


\section{METHODS}

\section{Human plaque selection}

All experiments were conducted in agreement with the code for proper secondary use of human tissue in the Netherlands (http://www.fmwv.nl). PHD2 protein expression was assessed by immunohistochemistry in human carotid autopsy samples $(n=38$, mean age 72 years, $64 \%$ men) representing the following stages of atherosclerosis: intimal thickening, pathological intimal thickening, thick fibrous cap (stable) atheroma, and plaque with intraplaque hemorrhage. PHD2 mRNA expression in 21 thick fibrous cap atheromas (stable) and 23 ruptured plaques was analyzed by microarray derived from human carotid endarterectomy, as described in (9). PHD2 mRNA expression intensities from microarrays was correlated with morphometrically analyzed histological plaque characteristics: plaque size, lipid core, necrotic core (\% of plaque), hemorrhage (\% of plaque), microvessels (\%CD31 of plaque), macrophages (\%CD68 of plaque), T-cells (\%CD3 of plaque). Classification of all used plaques was performed on hematoxylin and eosin (HE) stained slides according to Virmani et al. (10) by two investigators independently (T.L.T. and S.F.).

\section{Experimental animals}

All animal experiments were approved by the Dutch regulatory authorities and performed in accordance with the Dutch governmental guidelines. PHD2 cKO $(\mathrm{fl} / \mathrm{fl})$ mice were provided by Carmeliet (8) and crossed with LysMCre provided by Förster (11). The resulting $\mathrm{CKO} \mathrm{PHD}^{\mathrm{fl} / \mathrm{fl}} \mathrm{LysMCre}^{+/-}$mice were crossed with $\mathrm{LDLr}^{-/}$mice from an in house breeding colony (referred to as PHD2 cKO, Charles River). All mice were crossed back on a C57Bl6 background at least nine times. LysMCre ${ }^{+/-} \mathrm{LDLr}^{-/}$mice served as control in all experiments (referred to as PHD2 WT). For atherosclerosis experiments, only male mice were used ( $n=20$ per group). Animals were housed in the laboratory animal facility of Maastricht University under standard conditions. Food and water were provided ad libitum during the entire experiment.

\section{Atherosclerosis induction and plaque size measurement}

Atherogenesis was induced by feeding a Western-type diet: $0.25 \%$ cholesterol (Special Diet Services, \#824171, 15\% cocoa butter, 10\% maize starch, 20\% casein, $40.5 \%$ sucrose, $5.95 \%$ cellulose) for 6 or 12 weeks. The mice were euthanized by an intraperitoneal pentobarbital overdose $(100 \mathrm{mg} / \mathrm{kg})$ and subsequent blood draw from the right ventricle for hematopoietic parameters, flow cytometry, plasma cholesterol and plasma triglyceride determination. The remaining blood was removed by perfusion with PBS-sodium nitroprusside dehydrate. The aortic root and brachiocephalic artery were isolated and fixed in $1 \%$ paraformaldehyde.

Serial sections $(4 \mu \mathrm{m})$ from the paraffin embedded aortic root and brachiocephalic artery were cut and HE stained for plaque size measurement. Plaque size was measured in five representative sections of the aortic root and six sections of the brachiocephalic artery using computerized morphometry (Leica QWin V3, Cambridge, UK). Morphometry was performed by two blinded researchers independently. Necrotic core was defined as acellular and a-nuclear plaque area containing cholesterol clefts. 


\section{Immunohistochemistry}

Mouse atherosclerotic plaques were characterized for macrophage content (Mac3+ area/plaque area, BD Cat. No. 553322), smooth muscle cell content ( $\alpha \mathrm{SMA}^{+}$area/ plaque area, $\alpha$ SMA Sigma F3777), adventitial microvessel density (CD31 ${ }^{+}$microvessels/adventitial area, BD Cat. No. 550274), collagen type I (Coll-I ${ }^{+}$area/ plaque area, Abcam ab21286), collagen (picosirius red area/plaque area, analyzed with polarized light microscopy, Polyscience 09400), HIF1 $\alpha$ (Novus Biologicals, NB100-449), glycosaminoglycans (alcian blue $^{+}$area/ plaque area, Sigma A5268) and cartilage (safranin O). Sections were subjected to heat-induced antigen retrieval (PHD2, MAC3, Coll-I) or trypsin digestion (CD31) if needed, and specific antigen-antibody binding visualized by appropriate secondary antibodies and diaminobenzamidin (DAB), unless specified otherwise. In murine plaques, aSMA (DAKO M0851) and Ki67 (Abcam ab15580) doublestaining was done to measure SMC proliferation. Thickness of the fibrous cap was measured on $\alpha$ SMA-staned sections of the brachiocephalic artery (at least three continuous sections) and in the aortic root (two sections per plaque) as described in (12) using ImageJ. Murine PHD2 expression (Novus Biologicals NB100-2219) was visualized using streptavidin Dyelight 594 (Jackson Laboratories 016-510-084), and DAPI nuclear staining (Sigma D9542). Human plaques were serially stained with PHD2 (Novus Biologicals NB100-2219) and CD68 (DAKO M0814).

\section{Multispectral imaging}

Multispectral imaging (MSI) was performed as described (5) to analyze murine and human PHD2 expression, and PHD2-CD68 and $\alpha$ SMA-ki67 co-localization aSMA. Spectral images were taken between 420-720 nm (10 nm interval) at a 10x (human) and 20x (mouse) (plan apo) magnification using a Nuance spectral imaging system (Perkin Elmer/Caliper Life Sciences, Hopkinton, MA, USA) mounted on a Leica DM4000 B LED microscope, in case of immunofluorescence by using filters for N21 and A. Single stained sections, one chromogen/fluoro-chrome or counter stain only (DAB, Vector Red, Vector Blue, Dyelight 594, hematoxylin, DAPI), were used to create a spectral library. Computational decomposition of the individual image components was performed using the spectral library and NuanceTM 3.0.2 software. In case of aSMA/Ki67 double staining co-localization was assessed on a pixel-based measurement of the individual markers per plaque area using the same software.

\section{Blood count and flow cytometry}

White blood cells, red blood cells and platelets counts, hematocrit, hemoglobin, mean cell volume, mean cell hemoglobin, and mean corpuscular hemoglobin were measured using a Coulter Counter (Beckman Coulter, Woerden, NL). Immune cell subsets were defined in blood using flow cytometry (see supplemental figure 6 for gating strategy), and the TrueCount (BD, Cat No 340334) system to obtain absolute cell counts. Prior to antibody labeling the blood was subjected to erythrocyte lysis. Combinations of different antibodies were used to identify leucocytes (CD45 Biolegend clone 30-F11), B-cells (B220 BD clone RA3-6B2), T-helper cells (CD3 eBioScience clone 17A2/CD4 BD clone GK1.5), cytotoxic Tcells (CD3/CD8 eBioScience clone 53-6.7), NK cells (CD8/NK1.1 BD clone PK136) NKT cells (CD3/NK1.1), monocyte subsets (Ly6C Milteni clone 1G7.G10/CD11b BD clone M1/70) and granulocytes (Ly6G BD clone 1A8/CD11b). 


\section{Cell culture}

Bone marrow was isolated and cells were cultured for seven days in RPMI-1640 (Gibco with Glutamax, 2g/L glucose) supplemented with $10 \%$ FCS, 100U/ml PenicillinStreptomycin, and 15\% L929-conditioned medium to generate bone marrow-derived macrophages (BMDM).

Primary vascular smooth muscle cells (SMC) were isolated by enzymatic digestion ( $4 \mathrm{~h}$ at $37^{\circ} \mathrm{C}$ in DMEM with $3 \mathrm{mg} / \mathrm{ml}$ collagenase (Sigma, Zwijndrecht, the Netherlands) and 1 $\mathrm{mg} / \mathrm{ml}$ elastase (Sigma, Zwijndrecht, the Netherlands)) from aortas of C57Bl6 mice (after removal of endothelium and adventitia) and cultured in DMEM (Gibco) supplemented with $10 \%$ FCS and $100 \mathrm{U} / \mathrm{ml}$ Penicillin-Streptomycin.

Macrophage conditioned medium was obtained by culturing differentiated BMDM for $48 \mathrm{~h}$ in RPMI-1640 (Gibco with Glutamax, 2g/L glucose) supplemented with 1 or $10 \%$ FCS depending on the assay, 100U/ml Penicillin-Streptomycin, and 15\% L929-conditioned medium.

\section{Real time quantitative PCR}

In vitro experiments for gene expression analysis were performed in quadruplicate and repeated twice. Total RNA was isolated and transcribed as described (13). QPCR analyses were performed from $10 \mathrm{ng}$ cDNA using SYBR green (Biorad) and gene specific primer sets (Eurogentec, Liege, Belgium, Supplemental Table 1). Two housekeeping genes (cyclophilin, $18 \mathrm{~S}$ rRNA) insensitive to changes in $\mathrm{O}_{2}$ were used to correct for differences in mRNA levels between samples.

\section{Macrophage polarization measurement}

Macrophage polarization markers were measured using qPCR (Supplemental Table 1) and enzyme-linked immunosorbent assays (ELISA) for TNF- $\alpha$, IL12 and IL10 secretion (all from Invitrogen). ELISAs on conditioned medium from stimulated macrophages (PBS, LPS, IL4 or IL10) were performed according to manufacturer's protocol. Nitric oxide (NO) production was measured in conditioned medium using Griess reagent $\left(2.5 \% \mathrm{H}_{3} \mathrm{PO}_{4}, 1 \%\right.$ sulfanilamide, and $0.1 \%$ naphthalene diamine dihydrochloride). Analysis was performed using a microplate reader (Bio-Rad) at $450 \mathrm{~nm}$ (for ELISA) and at $550 \mathrm{~nm}$ (for NO assay).

\section{Western blot}

Cell pellets of BMDM were homogenized in an equal volume of RIPA buffer with EDTA-free protease inhibitor cocktail (Roche). The protein concentration was determined using BCA kit (Thermo Fisher Scientific, Cat. No. 23227), subsequently separated by $10 \%$ SDS-PAGE and transferred to a nitrocellulose membrane. Target proteins were detected with primary antibodies against HIF1 $\alpha$ (Novus Biologicals, NB100-449), HIF2 $\alpha$ (Novus Biologicals, NB100-122) and $\beta$-actin as loading control (MP biochemical, 691001), followed by secondary antibody incubation (Cell signaling, 7074 or Jackson Laboratories 715-035150) and chemiluminescent detection (SuperSignal West Femto, ThermoScientific, 34095) on a digital scanner.

\section{Collagen synthesis assay}

Collagen synthesis by BMDM and primary murine vascular smooth muscle cells (SMC) was evaluated after stimulation with BMDM-conditioned medium using Sirius red. SMC $\left(2 \times 10^{5}\right)$ 
were incubated for 96 hours with BMDM-conditioned RPMI medium. Macrophage collagen synthesis was measured after 72 hours with the last 48 hours under hypoxia $\left(0.2 \% \mathrm{O}_{2}\right)$, in 24 wells plates with a density of $0.4 \times 10^{6}$ cells per well. Collagen was visualized by Sirius red staining ( $1 \%$ Sirius red in $0.01 \mathrm{M} \mathrm{HCl}$ for one hour at RT), after fixation with $3.7 \%$ formaldehyde for $1 \mathrm{~h}$ at RT. The amount of collagen was spectrophotometrically determined in cell lysates at $544 \mathrm{~nm}$.

\section{SMC proliferation assay}

Proliferation of SMC in response to macrophage conditioned medium (see above) was measured on an ACEA xCELLigence (Roche). Primary SMC $\left(2 \times 10^{4}\right.$ cells) were seeded on a gold electrode implemented in a 96 wells plate and allowed to attach for 24 hours. After starvation for $12 \mathrm{~h}$ in RPMI-1640 (Gibco with Glutamax, $2 \mathrm{~g} / \mathrm{L}$ glucose) containing $0.5 \%$ FCS, BMDM-conditioned medium was added to the cells and incubated for 96 hours. Impedance was measured hourly and used to quantify proliferation (slope of impedance increment over time) using RCTA software (version 1.2, Roche).

\section{Smooth muscle cell migration assay}

Migration stimulation in primary murine SMC by conditioned macrophage medium of WT and PHD2 cKO was measured on an ACEA xCELLigence (Roche). SMC were starved in RPMI-1640 (Gibco with Glutamax, 2g/L glucose) containing 0.5\% FCS for 24 hours. Upper chambers of ACEA CIM 16 plates (ACEA, 20131122) were coated with 10ug/ml collagen $G$ (Biochrom, L7213) for one hour per side prior to start of the experiment. Subsequently, lower chambers were equilibrated for the respective conditioned mediums and controls. BMDM-conditioned medium contained a final concentration of $1 \%$ FCS and $15 \%$ LCM. SMC ( $4 \times 10^{4}$ cells) were then added to the upper chamber and migration was quantified in hourly measurements over time (during 24 hours) using the slope of the impedance increment over time.

\section{Collagen degradation assay}

MMP activity was determined using OmniMMP ${ }^{\mathrm{TM}}$ fluorogenic substrate (Enzo Life Science, BML-P126-0001). The substrate (Mca-Pro-Leu-Gly-Leu-Dpa-Ala-Arg-NH2) incorporates a quencher (Dpa) and a fluorescent side (Mca). MMP cleavage of the quencher will yield an increase in fluorescence. A total of $0.14 * 10^{6}$ BMDM (lysed in PBS containing $1 \%$ TritionX100) were incubated with $180 \mathrm{mM} \mathrm{CaCl}_{2}$ and $65 \mu \mathrm{M}$ OmniMMP substrate at $37^{\circ} \mathrm{C}$ and the fluorescence was detected at an interval of two minutes up to 300 minutes on a Spectromax (Ex 328nm, Em 393nm, Molecular Devices SPECTRAmax M2).

\section{Plasma lipid measurements}

Plasma was separated by centrifugation, and stored at $-80^{\circ} \mathrm{C}$ until further use. Standard enzymatic techniques were used to assess plasma cholesterol (CHOD-PAP method Cholesterol FS Ecoline product no. 113009990314, DiaSys, Holzheim, GE) and plasma triglycerides (FS5' Ecoline REF 157609990314; DiaSys - Diagnostic Systems GmbH, Holzheim, Germany) automated on the Cobas Fara centrifugal analyzer (Roche). 


\section{Statistical analysis}

All data are presented as mean \pm SEM. Shapiro-Wilk test for normal distribution was performed, followed by a Grubbs outlier test. Finally, groups were compared with student's t-test or Mann-Whitney rank-sum test. (GraphPad Prism4, La Jolla, CA, USA). A $\mathrm{p}$-value of $\mathrm{p}<0.05$ was considered significant $\left(* p<0.05,{ }^{*} p<0.01, * * * p<0.001\right)$. 


\section{RESULTS}

PHD2 expressed in human plaques, mainly in macrophages

PHD2 protein and mRNA was detected in human atherosclerosis however, expression levels were similar in stable versus ruptured human atherosclerotic plaques (Figure $1 \mathrm{~A}-\mathrm{B}$ ). Protein expression was present in macrophages, endothelial cells and smooth muscle cells, but protein correlated mostly with macrophages (Figure 1A). Similarly, mRNA expression of PHD2 correlated with CD68 positivity in adjacent immunohistochemically stained plaque locations (Table 1). 

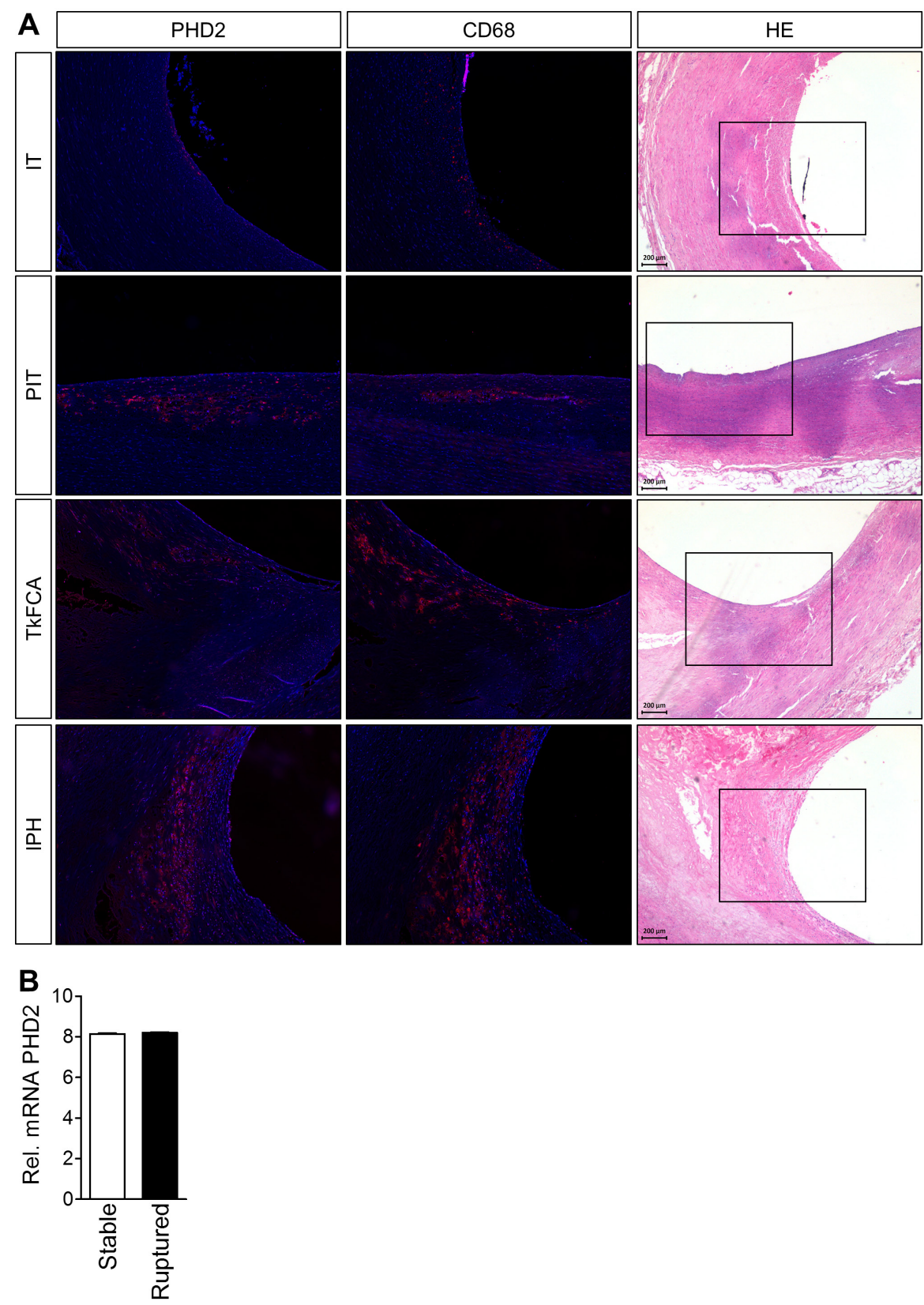

Figure 1. PHD2 expression in human plaques, co-localized with CD68+ macrophages

(A) PHD2 and CD68 protein expression (both in red) in different stages of human atherosclerosis, HE for overview (samples from autopsy; IT=intmal thickening, PIT=pathological intimal thickening, TkFCA=thick fibrous cap atheroma, IPH=intra plaque hemorrhage). (B) PHD2 mRNA expression (derived from microarrays) in stable and ruptured human carotid endarterectomy plaques ( $n=21$ for stable, unstable $n=23$ ). PHD2 and CD68 100x magnification, HE insert 100x magnification, picture 50x magnification. 


\begin{tabular}{lll}
\hline PHD2 versus: & Regression Coefficient & P-value \\
\hline Plaque size & -0.3579 & 0.7204 \\
Lipid Core & 157063752194667.0000 & 0.1163 \\
Necrotic core (\%) & 151417511136095.0000 & 0.1300 \\
Hemorrhage (\%) & 10053255065528.0000 & 0.3147 \\
CD31 (\%) & 114598631697176.0000 & 0.2518 \\
CD68 (\%) & 196932166967027.0000 & $0.0489 *$ \\
CD3 (\%) & 111014971234584.0000 & 0.2669 \\
\hline
\end{tabular}

Table 1. PHD2 mRNA expression in human plaques correlates with plaque macrophages Correlation of PHD2 mRNA expression (derived from microarray) with histological features in neighboring plaque areas. Samples were prepared from human carotid endarterectomy samples ( $n=44$ patients). ${ }^{*} \mathrm{P}<0.05$.

\section{Effective myeloid PHD2 knock out in atherosclerotic mice}

Although hypoxia and HIF expression have been demonstrated in murine lesions (5), the expression pattern of PHD2 in murine atherosclerosis is unknown. Here, we show the expression of PHD2 in macrophages, EC and SMC of murine plaques (Figure 2A). To study macrophage specific PHD2 deficiency, PHD2 cKO $\mathrm{LDLr}^{-/}$mice were used. PHD2 cKO reduced PHD2 levels in macrophages in vivo and in BMDM in vitro (Figure 2A, B). Since PHD2 is the main regulator of HIF1 $\alpha$ stability, higher HIF1 $\alpha$ levels were observed in PHD2 cKO macrophages and plaques (Supplemental figure 1A-B). HIF2 $\alpha$ protein was unchanged (Supplemental figure 1A). Thus, PHD2 cKO mice show a functional PHD2 knock down.

\section{Conditional PHD2 knock out increased plaque size without systemic side effects}

To study macrophage specific PHD2 deficiency, atherosclerosis was induced in PHD2 cKO $\mathrm{LDLr}^{-1}$ mice by feeding a Western-type diet for 6 or 12 weeks. PHD2 cKO increased plaque size in all examined arteries and all time points (Figure $2 \mathrm{C}$ left panel): fatty streaks in the aortic root after 6 weeks, intermediate plaques in the brachiocephalic artery after 12 weeks, and advanced plaques in the aortic root after 12 weeks of Western type diet. In all three locations, no difference in necrotic core percentage (AR 6 weeks $1.6 \%$ vs $2.7 \%$; BC 12 weeks $4.9 \%$ vs $5.9 \%$; AR 12 weeks $12.7 \%$ vs $12.5 \%$ ) could be found between PHD2 cKO and WT (Figure $2 \mathrm{C}$ right panel).

The potent decrease in PHD2 levels did not affect body weight or plasma lipid levels, except a slight decrease in plasma cholesterol level at 12 weeks (Supplemental Figure 2CE). Flow cytometry revealed no difference in immune cell counts (Supplemental figure 2). Also total red blood cells, hemoglobin and hematocrit were normal in the PHD2 cKO mice (Supplemental figure 3), despite HIFs known role in erythropoiesis. An experienced laboratory animal pathologist (M.G.) did not detect any macroscopic or microscopic abnormalities in any major organ (data not shown). 

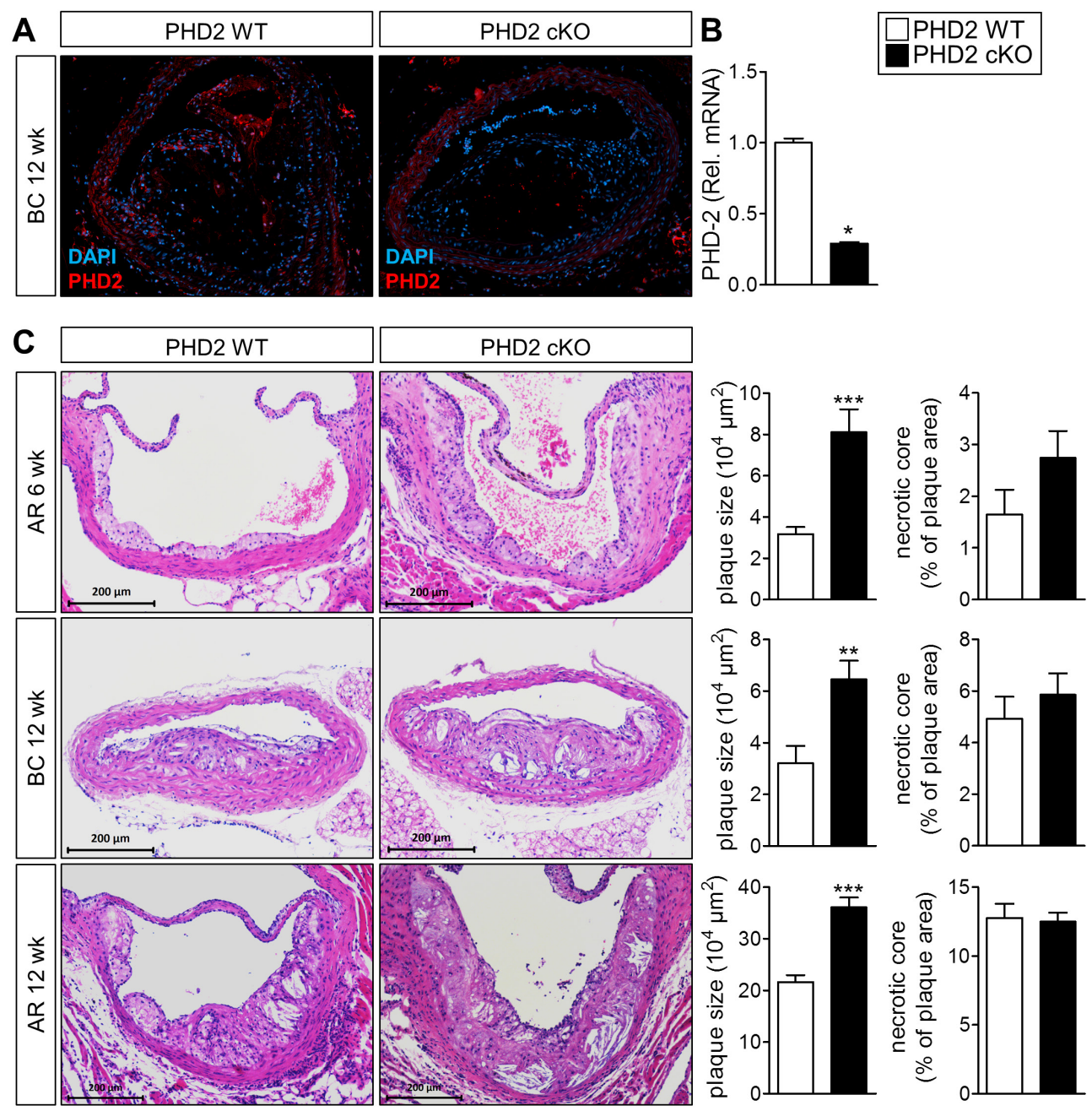

Figure 2. Myeloid PHD2 cKO increased plaque size

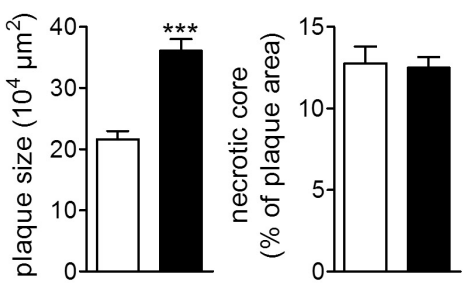

(A) Immunofluorescent staining for PHD2 expression in murine plaques (left) and PHD2 cKO (right). (B) PHD2 mRNA expression in PHD2-cKO $(n=4)$ compared to PHD2-WT $(n=4)$ BMDM. (C) Plaque size in PHD2 cKO mice in all analyzed locations: early plaques in the aortic root after 6 wks of HCD (upper panel, n=17 for PHD2-WT, n=19 for PHD2-cKO), intermediate plaques in the brachiocephalic artery after 12 wks of Western diet (middle panel, $n=18$ for PHD2-WT, n=16 for PHD2-cKO) and advanced plaques in the aortic root after 12 weeks of diet (lower panel, $\mathrm{n}=17$ for PHD2-WT, $\mathrm{n}=17$ for PHD2-cKO). Quantification of the necrotic core in all stages (right panel). Mean \pm SEM $* \mathrm{P}<0.05, * * \mathrm{P}<0.01, * * * \mathrm{P}<0.001$.

\section{Increased plaque size was not related to angiogenesis}

As myeloid specific PHD2 downregulation is known to induce angiogenesis (8), CD31 positive microvessels in plaque and adventitia were quantified. PHD2 cKO was not able to boost the paucity of intra plaque microvessels common to murine plaques: no CD31+ microvessels were observed in any arterial bed (Figure 3A). In addition, no difference in adventitial microvessel density was found (Figure $3 \mathrm{~A}$ ), despite the enhanced expression of pro-angiogenic growth factors (VEGF-A and Ang-1, Figure 3B) by isolated macrophages in vitro. Likewise, maturation of the adventitial microvessels (aSMA-surrounded 64 
microvessels) was similar (Figure 3C), Moreover, secondary effects of plaque angiogenesis were not observed either, as Perls iron staining did not detect iron deposition resulting from extravasated, degraded red blood cells (data not shown). Also, angiogenesis was not enhanced in any other major organ (data not shown). Thus, despite the previously reported high angiogenic potential of PHD2 cKO in other mouse models of vascular disease, we were unable to find changes in atherosclerosis-related angiogenesis.
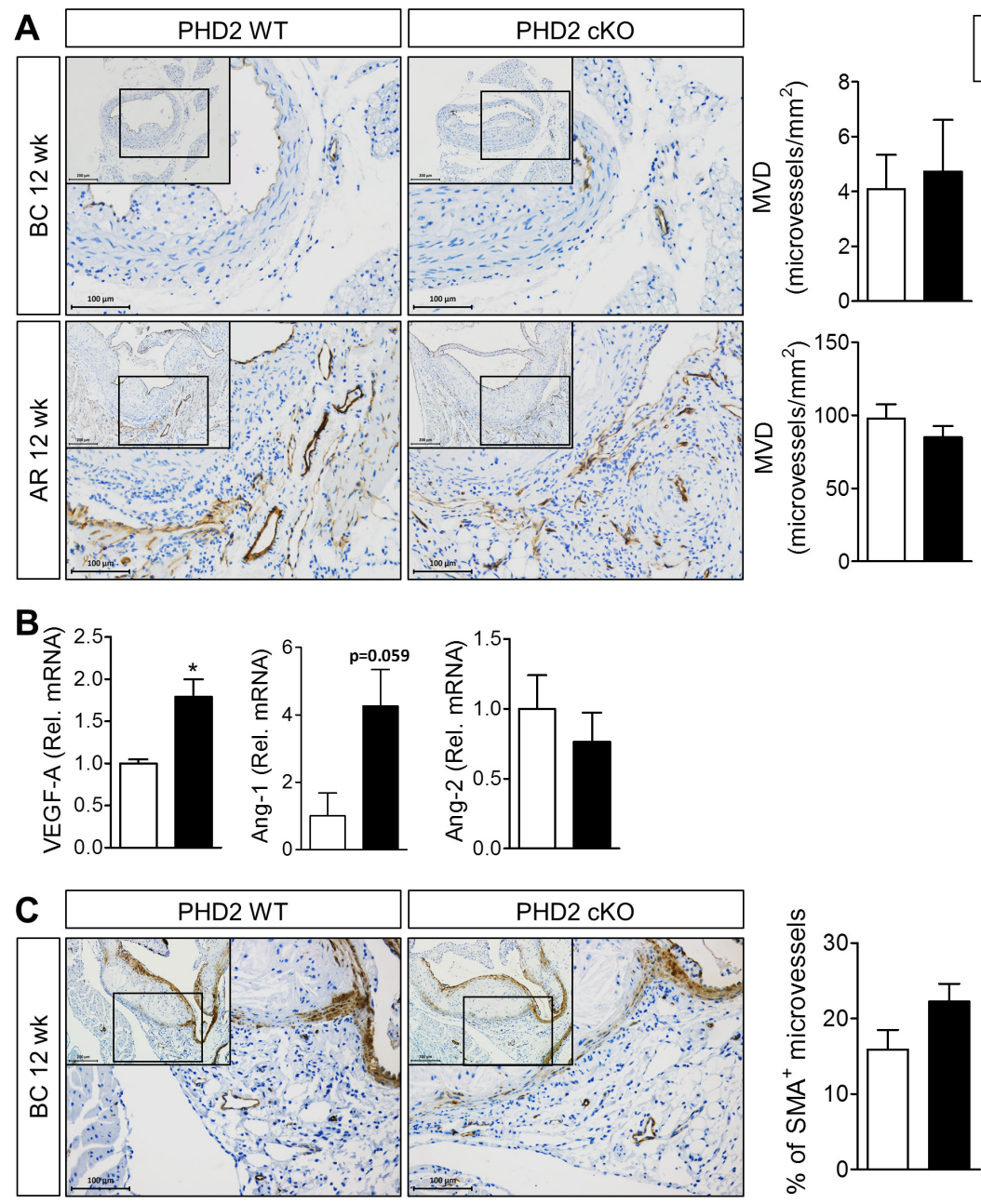

Figure 3. PHD2 cKO does not affect adventitial microvessel density

(A) Quantification of the adventitial CD31+ microvessel density underlying plaques of the brachiocephalic artery (for both $\mathrm{n}=17$ ) and aortic root (for both $\mathrm{n}=15$ ) after 12 wks of Western diet. (B) Gene expression of angiogenic factors in PHD2 cKO BMDM (VEGF $n=4$, Ang-1 $n=4$, Ang-2 $n=3$ ). (C) Maturation status of adventitial plaque associated microvessels in the aortic root after 12 wks of HCD $(n=14)$. Insert 100x magnification, zoom picture 200x magnification. Mean \pm SEM *P $<0.05$ 


\section{Conditional PHD2 knock out increased plaque stability via increased collagen}

Plaques of PHD2 cKO mice contained an increased amount of collagen (Figure 4A), specifically type I collagen (Supplemental figure $4 \mathrm{~A}$ ). The increase in collagen resulted in a significantly thickened cap in intermediate and advanced plaques of the PHD2 cKO mice (Figure 4B). Also, glycosaminoglycans, reflecting extracellular matrix components, were increased in PHD2 cKO plaques (Supplemental figure 4B). Staining for cartilage revealed that no mature cartilage or chondrocytes were present in any of the plaques (Supplemental figure 4C). Overall, the increased total collagen and cap thickness together with the unchanged necrotic core size (Figure $2 \mathrm{C}$ right panel) suggest improved plaque stability by PHD2 cKO despite the increase in atheroma size. 

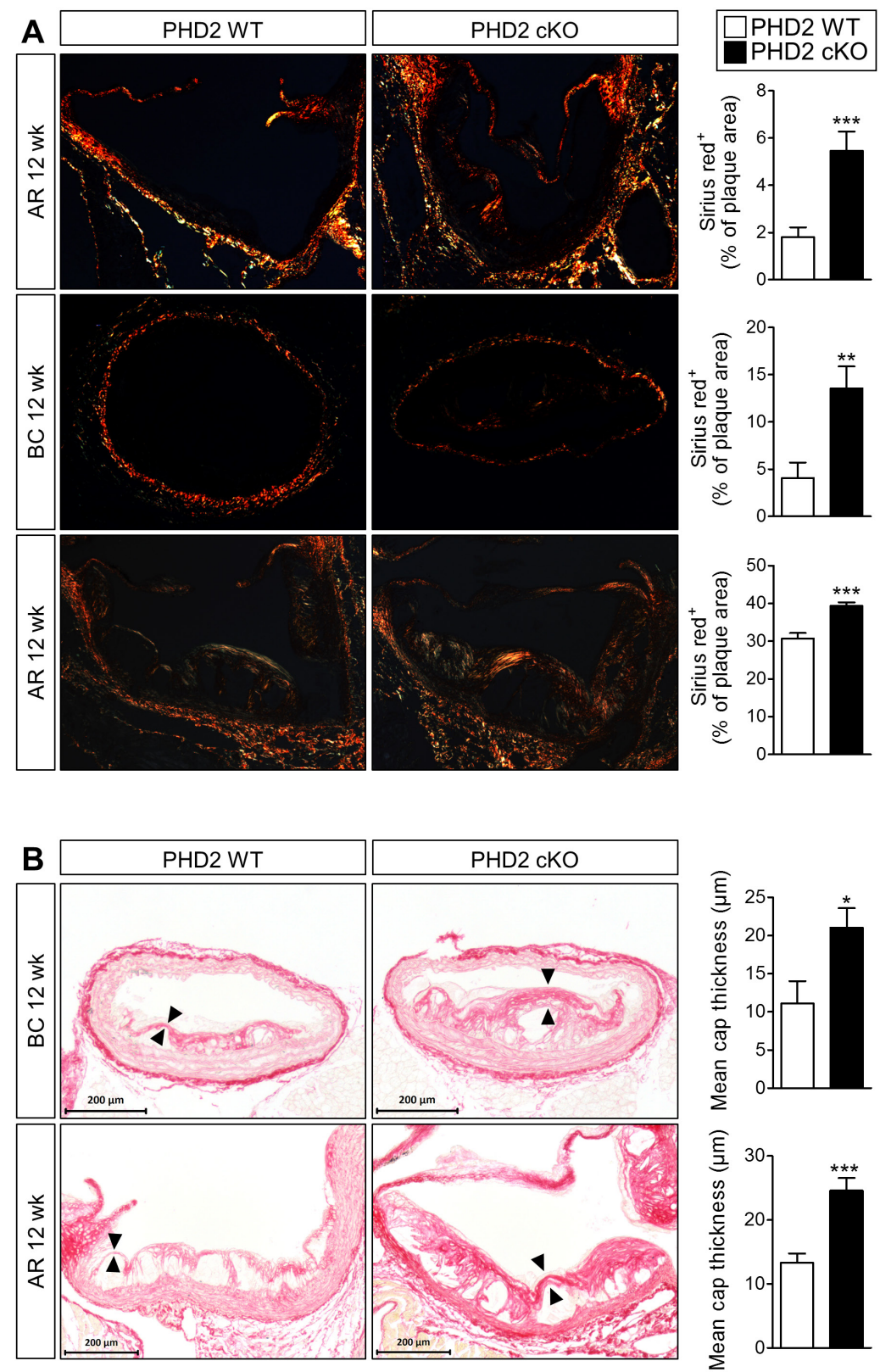

Figure 4. Increased plaque collagen and cap thickness by PHD2 cKO

(A) Total plaque collagen content of early plaques (upper panel), intermediate plaques and advanced plaques (lower panel) visualized by Sirius red staining and polarized light. (B) Mean cap thickness measurement of Sirius red stained sections in intermediate (upper panel) and advanced plaques (lower panel). Mean \pm SEM $* P<0.05$, $* * \mathrm{P}<0.01, * * * \mathrm{P}<0.001$. 


\section{PHD2 cKO macrophages account for increased collagen}

Collagen content depends on SMC-dependent synthesis and macrophage-dependent collagen degradation. Macrophages degrade collagen through the expression of matrix metalloproteinases (MMPs). The observed increase in plaque collagen content could thus be explained by a reduction in plaque macrophages and/or enhanced macrophage collagen degradation capacity. Indeed, macrophage content was significantly decreased in early and advanced plaques of PHD2 cKO mice, and a similar trend was observed in intermediate plaques (Figure 5A). Macrophage MMP activity and expression of the MMPs most relevant for murine atherosclerosis in vitro was unaltered by PHD2 cKO (14) (Figure $5 B, C)$. Similarly, the expression of major MMPs inhibitors, TIMP2 and 3 did not differ (Figure $5 \mathrm{C}$ ). In addition, a clear in vitro M1-M2 polarization prolife is lacking (Supplemental Figure 5). However, arginase is enhanced, which is mostly associated with fibrosis (15). Together, despite the similar degradation capacity per macrophage, an absolute reduction in collagen-degrading macrophages is expected to yield a net reduction in collagen degradation.

Macrophages are also capable of producing slight amounts of collagen (16) and direct collagen production by PHD2 cKO macrophages in vitro was enhanced compared to WT macrophages (Figure 5D). Notably, the production of collagen by WT macrophages was enhanced by hypoxic conditions, while hypoxia did not further enhance collagen production by PHD2 cKO macrophages (Figure 5D). This suggests that hypoxia and PHD2 have overlapping transcriptional effects, likely mediated by HIF. Indeed, transcription of collagen prolyl 4-hydroxylase alpha (P4HA) 1, a key enzyme of the collagen production cascade regulated by HIF1 $\alpha$ (17), was upregulated by PHD2 cKO in a hypoxia-dependent way (Figure $5 \mathrm{E}$ ). In contrast, $\mathrm{P} 4 \mathrm{HA} 2$ expression was dependent on synergistic, but not overlapping signaling pathways induced by hypoxia and PHD2. Together, PHD2 cKO stimulated macrophage collagen production in vitro likely via P4HA1. However, in light of the reduction in macrophage content in vivo, a slight increase in collagen production by macrophages may not be completely sufficient to explain the accumulation of plaque collagen in PHD2 cKO mice. 
A

A
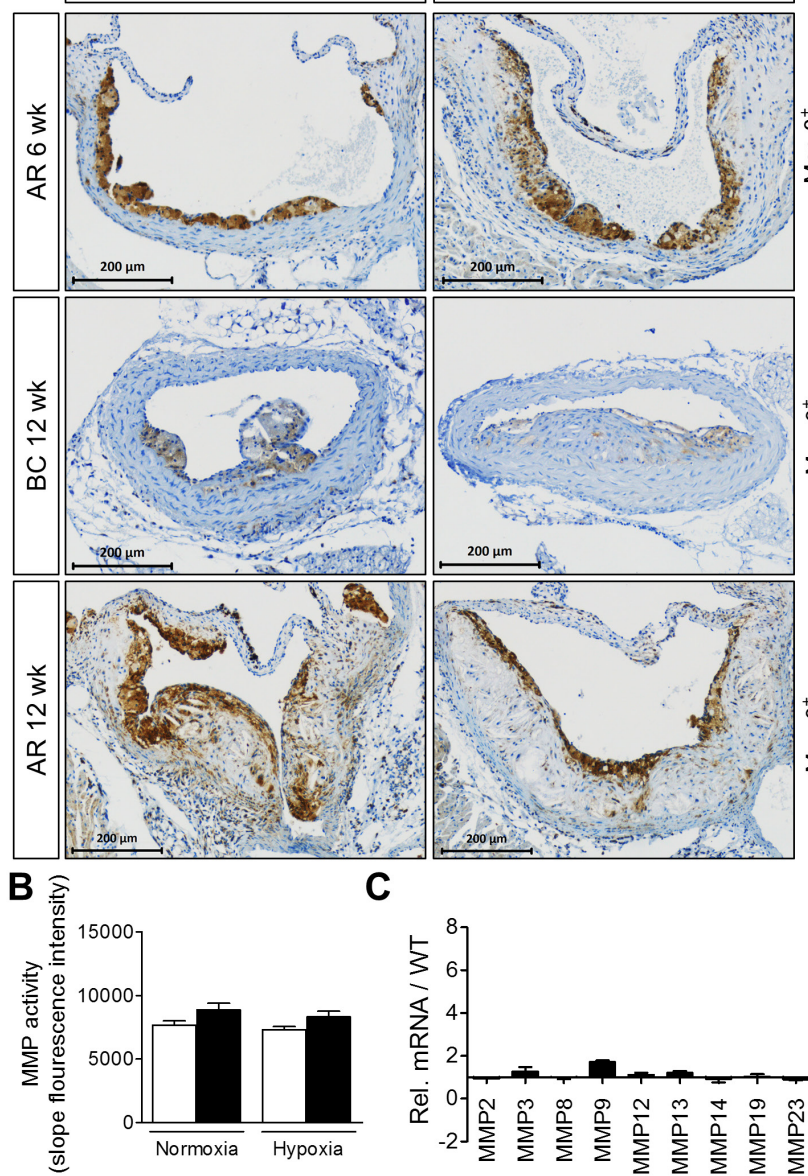

D

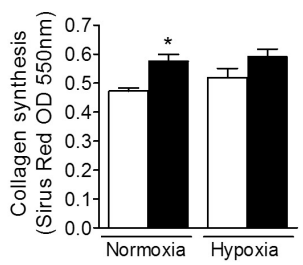

C

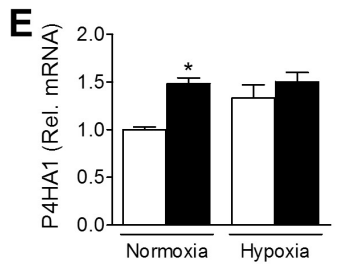

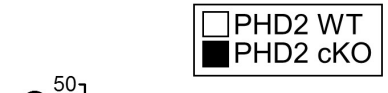
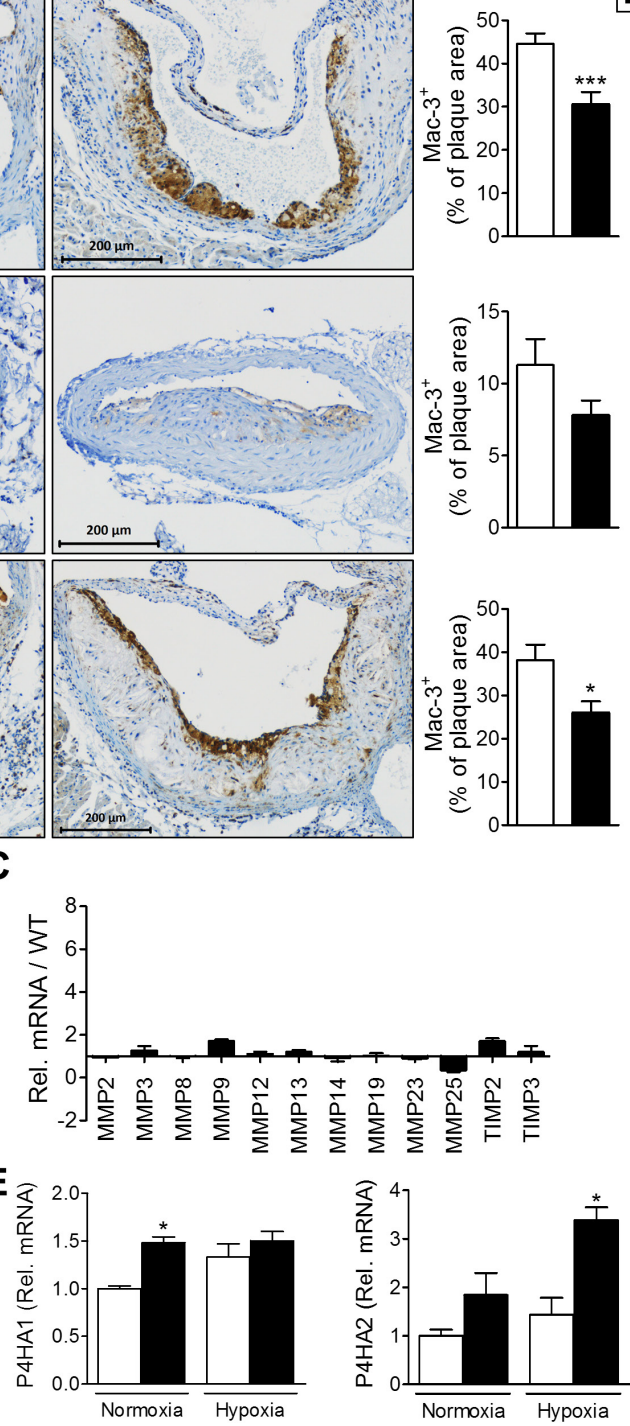

Figure 5. PHD2 cKO decreased macrophage content, but enhanced collagen synthesis, not degradation

(A) Percentage of Mac3 positive macrophages in early, intermediate and advanced plaques. (B) In vitro MMP activity in lysates of PHD2 WT and cKO BMDM ( $n=4$ for all conditions). (C) In vitro mRNA expression levels of relevant MMPs in PHD2 cKO BMDM compared to PHD2 WT BMDM ( $n=4$ for all). (D) In vitro macrophage collagen synthesis in PHD2 WT and PHD2 cKO BMDM ( $n=4$ for all conditions). (E) In vitro gene expression of collagen prolyl hydroxylases 1 and 2 in PHD2 WT and PHD2 cKO BMDM ( $n=4$ for all). Mean \pm SEM $* P<0.05, * * * P<0.001$.

\section{Increase in collagen independent of altered smooth muscle cell function}

Smooth muscle cells (SMCs) are the major source of collagen production in atherosclerosis. Net collagen deposition depends on increased SMC content, and/or enhanced SMC collagen production, which can both be regulated by paracrine signaling through factors secreted by macrophages. However, while aSMA positive SMC content 
was increased in early lesions, it was unchanged in intermediate plaques and even decreased advanced plaques (Figure 6A). The early increase in SMC content was not due to SMC proliferation in vivo (Figure $6 \mathrm{~B}$ ). SMC proliferation and migration in vitro in response to WT or PHD2cKO macrophage conditioned medium were also unchanged (Figure $6 \mathrm{C}, \mathrm{D}$ ). In addition, SMC collagen production was not altered by incubation with conditioned medium of PHD2 cKO macrophages (Figure 6E). However, the ratio of collagen-producing smooth muscle cells over collagen-degrading macrophages was enhanced in plaques of PHD2 cKO mice (Figure 6F). Overall, the enhanced $\mathrm{SMC} /$ macrophage ratio, together with a slight increase in collagen production by macrophages likely explains the accumulation of plaque collagen in PHD2 cKO mice. 
A
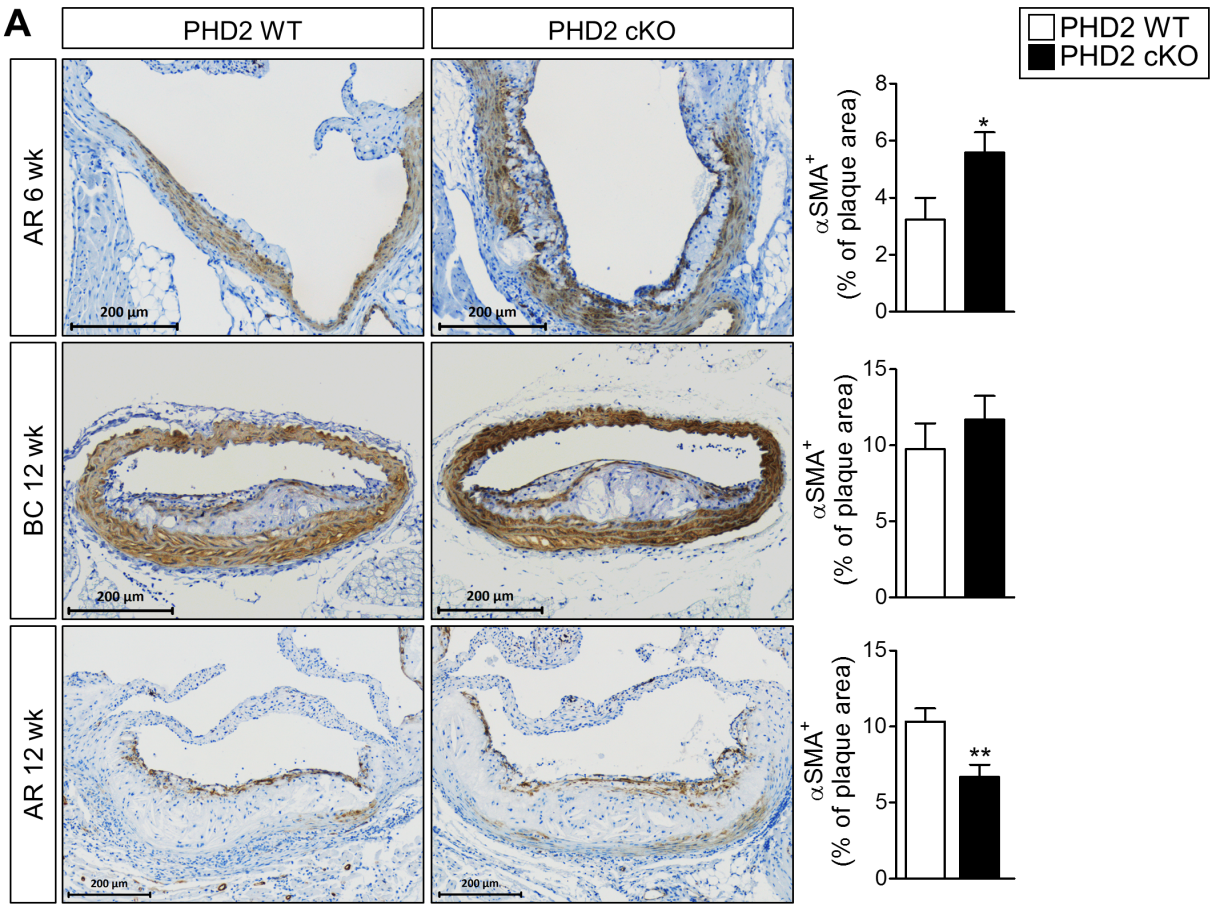

B PHD2 WT
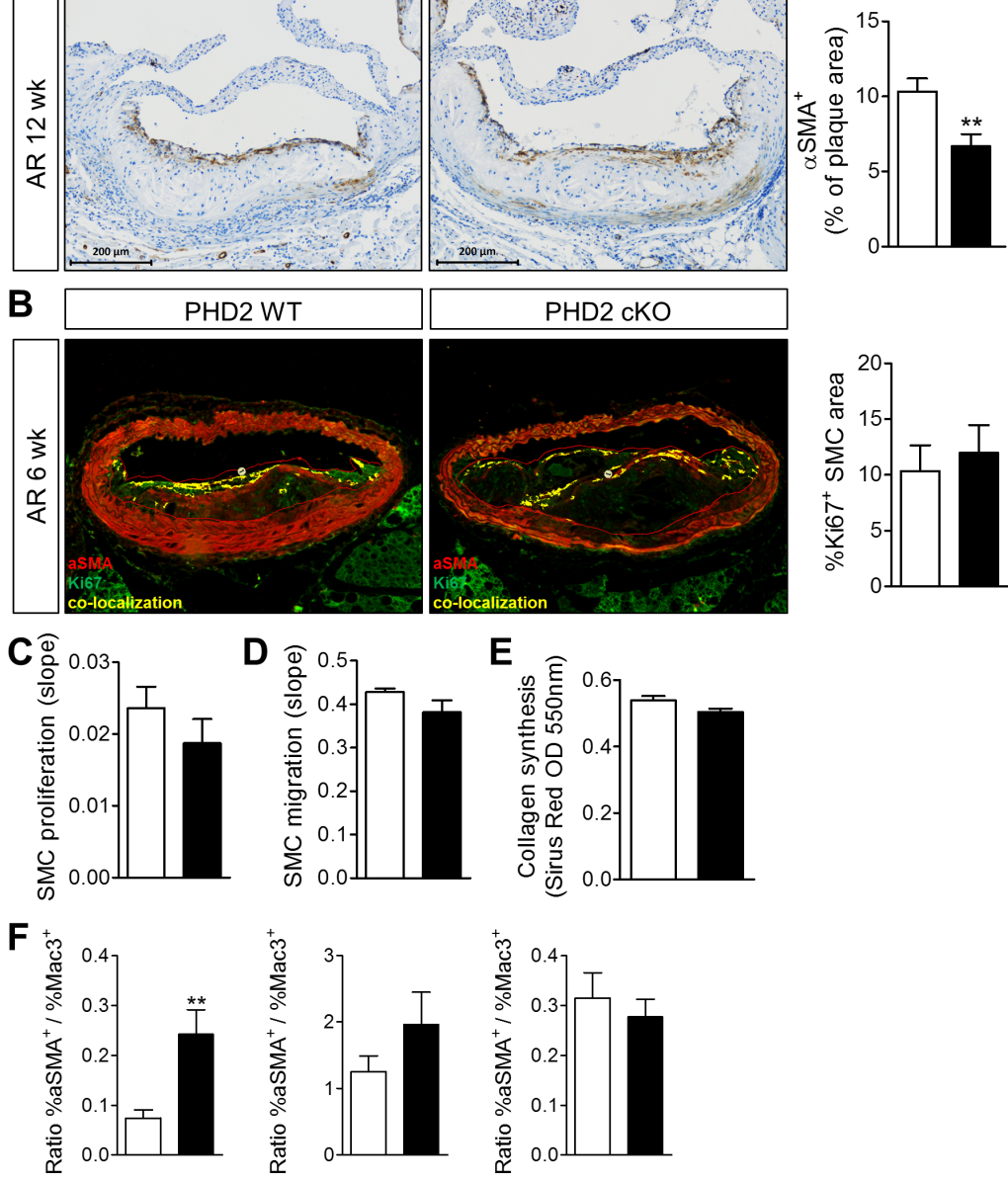

Figure 6. Increased SMC content in PHD2 cKO plaques but no difference in SMC functionality

(A) Percentage of $\alpha$ SMA positive SMC in early, intermediate and advanced plaques. (B) SMC proliferation in early and intermediate plaques. (C) SMC proliferation ( $n=4$ for both) (D) migration ( $n=4$ for both) and (E) collagen synthesis ( $n=4$ for both) in vitro after stimulation with WT or PHD2 cKO BMDM conditioned medium. (F) In vivo 
Chapter 3

ratio of SMC percentage to Mac3 percentage in early, intermediate and advanced plaques. Mean \pm SEM $* P<0.05$, $* * \mathrm{P}<0.01, * * * \mathrm{P}<0.001$. 


\section{DISCUSSION}

Myeloid PHD2 deficiency resulted in a remarkable pro-fibrotic phenotype of the atherosclerotic plaque, while plaque size was increased all throughout plaque progression (early and advanced plaques). Interestingly, the increase in plaque size could not be attributed to augmented angiogenesis. Rather, lesions had increased collagen content and a thickened fibrous cap, together with an unchanged necrotic core and reduced macrophage content, all features of a more stable plaque. PHD2 cKO thus firmly enhanced plaque stability.

\section{PHD2 deficiency shifts balance to collagen accumulation}

Collagen content is the resultant of a balance between production and degradation. This balance is dependent on macrophage and smooth muscle cell density, as well as their activity. As smooth muscle cells are major contributors to collagen production, the effect of paracrine signaling by PHD2 cKO macrophages on SMC function was studied. SMC collagen synthesis was unchanged. SMC density in vivo was only enhanced during the initiation phase of atherogenesis, and proliferation and migration were not affected by PHD cKO. Macrophages have been shown to play a major role in collagen homeostasis via MMP excretion (18). Although macrophage MMP activity was unaltered in PHD2 cKO macrophages, macrophage density in vivo was reduced. This may result in a net decrease of collagen degradation. Interestingly, collagen production by macrophage was increased. However, macrophage collagen production could only be assessed in vitro, making a definite conclusion difficult. Although macrophages represent the most frequent cell type within an atherosclerotic plaque and are capable of producing slight amounts of collagen, the overall contribution of macrophage collagen production to the total collagen production in atherosclerosis remains unknown (16). Thus, the increased ratio between smooth muscle cell and macrophage content, together with the slight increase in macrophage collagen production, most likely accounts for the net increase in plaque collagen.

\section{Myeloid PHD2 deficiency does not increase angiogenesis in atherosclerosis}

Unexpectedly, PHD2 cKO did not enhance microvessel density, nor maturation in plaques or other organs. This is in contrast to a previous report showing increased angiogenesis by macrophage PHD2 knockdown under acute ischemic conditions (8). The major difference between the present study and the previously reported study (8) are the use of homozygous $\mathrm{PHD}_{2}{ }^{\mathrm{fl} / \mathrm{fl}}-\mathrm{Cre}^{+/-}$versus heterozygous $\mathrm{PHD}^{\mathrm{fl} / \mathrm{wt}}-\mathrm{Cre}^{+/-}$mice. Macrophages derived from $\mathrm{PHD}^{\mathrm{fl} / \mathrm{fl}}$ mice did display an increase in the expression of pro-angiogenic cytokines, as did PHD2 ${ }^{\mathrm{fl} / \mathrm{wt}}$ macrophages. However, initiation of angiogenesis not only depends on EC proliferation, but is also critically dependent on EC migration and thus degradation of extracellular matrix. Here, the massive matrix production by PHD2 homozygous knockout mice, may simply have inhibited EC migration in vivo. Indeed, Takeda et al did not observe any changes in fibrosis, and PHD2 heterozygosity might have resulted in a different balance of pro-angiogenic and anti-fibrotic signaling. Nevertheless, homozygous PHD2 deficiency in macrophages does not appear to affect angiogenesis in atherosclerosis. 


\section{Therapeutic PHD2 inhibition in human atherosclerosis}

PHD inhibitors which are currently tested in clinical trials for the treatment of anemia in patients with chronic kidney disease (NCT02174731), might represent an accessible drug. Cardiovascular events might be an interesting secondary outcome of these clinical trials, elucidating the role of PHD inhibition in atherosclerosis. Although our data clearly suggest PHD2 inhibition as a potential way to stabilize plaques, one should be aware of the risk of enhancing plaque size. The macrophages in our model were PHD2 deficient from the beginning of atherosclerosis formation onwards, therefore the effect of PHD2 inhibition on established plaque burden and stabilization remains unclear.

The current PHD inhibitors are neither PHD isoform specific nor cell type specific. There are four PHD isoforms with varying biological functions. The general inhibition of all PHDs is therefore difficult to predict. In the current study only the myeloid compartment was PHD2 deficient, and orally administered PHD inhibitors would affect the entire body and evoke off-target effects. One possibility for targeted delivery of PHD inhibitors into plaques would be the packaging in HDL nanoparticles, which has been shown for statins recently (19). Thus, PHD2 inhibition might become a suitable target once isoform-specific and cell-targeted inhibitors are available to assess the effect of PHD2 inhibition on established plaque size and stability.

In conclusion, myeloid PHD2 deficiency dramatically enhanced plaque stability by autocrine and paracrine effects on macrophages and smooth muscle cells respectively, resulting in massive accumulation of plaque collagen. Future studies with macrophagetargeted therapy should thus exploit PHD2 inhibition to promote plaque stabilization. 


\section{ACKNOWLEDGEMENTS}

The authors want to thank Clairy Dinjens, Anique Janssen and Niko Deckers for their technical support. The authors also want to thank the staff of the animal facility at Maastricht University for their help with breeding of and providing care for the animals. The research was supported by a VENI fellowship of the Netherlands Organization of Scientific research (NWO, 016.116.017 to J.C.S.), a PhD-student fellowship from the Cardiovascular Research Institute Maastricht (to T.L.T.), the Dutch Heart Foundation (2011T6 to J.M.E.M.C., 2012T079 to M.M.P.C.D.) and ZonMW MKMD (114021004 to J.M.E.M.C.). 


\section{REFERENCES}

1. Murray CJL, Lopez AD. Measuring the global burden of disease. N Engl J Med. 2013 Aug 1;369(5):44857.

2. Bentzon JF, Otsuka F, Virmani R, Falk E. Mechanisms of plaque formation and rupture. Circ Res. 2014 Jun 6;114(12):1852-66.

3. Ribatti D, Levi-Schaffer F, Kovanen PT. Inflammatory angiogenesis in atherogenesis--a double-edged sword. Ann Med. 2008 Jan;40(8):606-21.

4. Marsch E, Sluimer JC, Daemen MJAP. Hypoxia in atherosclerosis and inflammation. Curr Opin Lipidol. 2013 Oct;24(5):393-400.

5. Marsch E, Theelen TL, Demandt JAF, Jeurissen M, van Gink M, Verjans R, et al. Reversal of hypoxia in murine atherosclerosis prevents necrotic core expansion by enhancing efferocytosis. Arterioscler Thromb Vasc Biol. 2014 Dec;34(12):2545-53.

6. Bosco MC, Puppo M, Blengio F, Fraone T, Cappello P, Giovarelli M, et al. Monocytes and dendritic cells in a hypoxic environment: Spotlights on chemotaxis and migration. Immunobiology. 2008 Nov;213(910):733-49.

7. Kaelin WG, Ratcliffe PJ. Oxygen sensing by metazoans: the central role of the HIF hydroxylase pathway. Mol Cell. 2008 May 23;30(4):393-402.

8. Takeda Y, Costa S, Delamarre E, Roncal C, Leite de Oliveira R, Squadrito ML, et al. Macrophage skewing by Phd2 haplodeficiency prevents ischaemia by inducing arteriogenesis. Nature. 2011 Nov 3;479(7371):122-6.

9. Goossens P, Gijbels MJJ, Zernecke A, Eijgelaar W, Vergouwe MN, van der Made I, et al. Myeloid type I interferon signaling promotes atherosclerosis by stimulating macrophage recruitment to lesions. Cell Metab. 2010 Aug 4;12(2):142-53.

10. Virmani R, Kolodgie FD, Burke AP, Farb A, Schwartz SM. Lessons from sudden coronary death: a comprehensive morphological classification scheme for atherosclerotic lesions. Arterioscler Thromb Vasc Biol. 2000 May;20(5):1262-75.

11. Clausen BE, Burkhardt C, Reith W, Renkawitz R, Förster I. Conditional gene targeting in macrophages and granulocytes using LysMcre mice. Transgenic Res. 1999 Aug;8(4):265-77.

12. Seimon TA, Wang Y, Han S, Senokuchi T, Schrijvers DM, Kuriakose G, et al. Macrophage deficiency of p38alpha MAPK promotes apoptosis and plaque necrosis in advanced atherosclerotic lesions in mice. J Clin Invest. 2009 Apr;119(4):886-98.

13. Sluimer JC, Kisters N, Cleutjens KB, Volger OL, Horrevoets AJ, van den Akker LH, et al. Dead or alive: gene expression profiles of advanced atherosclerotic plaques from autopsy and surgery. Physiol Genomics. 2007 Aug 20;30(3):335-41.

14. Newby AC. Metalloproteinases promote plaque rupture and myocardial infarction: A persuasive concept waiting for clinical translation. Matrix Biol. 2015 May;44-46:157-66.

15. Bhatta A, Yao L, Toque HA, Shatanawi A, Xu Z, Caldwell RB, et al. Angiotensin II-induced arterial thickening, fibrosis and stiffening involves elevated arginase function. Jourd'heuil D, editor. PLoS One. 2015 Mar 25;10(3):e0121727.

16. Weitkamp B, Cullen P, Plenz G, Robenek H, Rauterberg J. Human macrophages synthesize type VIII collagen in vitro and in the atherosclerotic plaque. FASEB J. 1999 Aug;13(11):1445-57.

17. Aro E, Khatri R, Gerard-O’Riley R, Mangiavini L, Myllyharju J, Schipani E. Hypoxia-inducible factor-1 (HIF1) but not HIF-2 is essential for hypoxic induction of collagen prolyl 4-hydroxylases in primary newborn 
mouse epiphyseal growth plate chondrocytes. J Biol Chem. 2012 Oct 26;287(44):37134-44.

18. Moore KJ, Tabas I. Macrophages in the pathogenesis of atherosclerosis. Cell. 2011 Apr 29;145(3):34155.

19. Tang J, Lobatto ME, Hassing L, van der Staay S, van Rijs SM, Calcagno C, et al. Inhibiting macrophage proliferation suppresses atherosclerotic plaque inflammation. Sci Adv. 2015 Apr;1(3). 


\section{SUPPLEMENTAL DATA}

\section{A}

HIF1a

HIF2 $\alpha$

\section{PHD2 WT PHD2 cKO}

$\beta$-actin

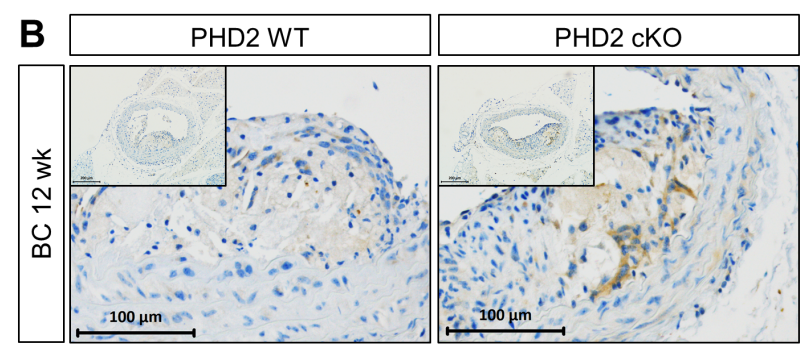

C
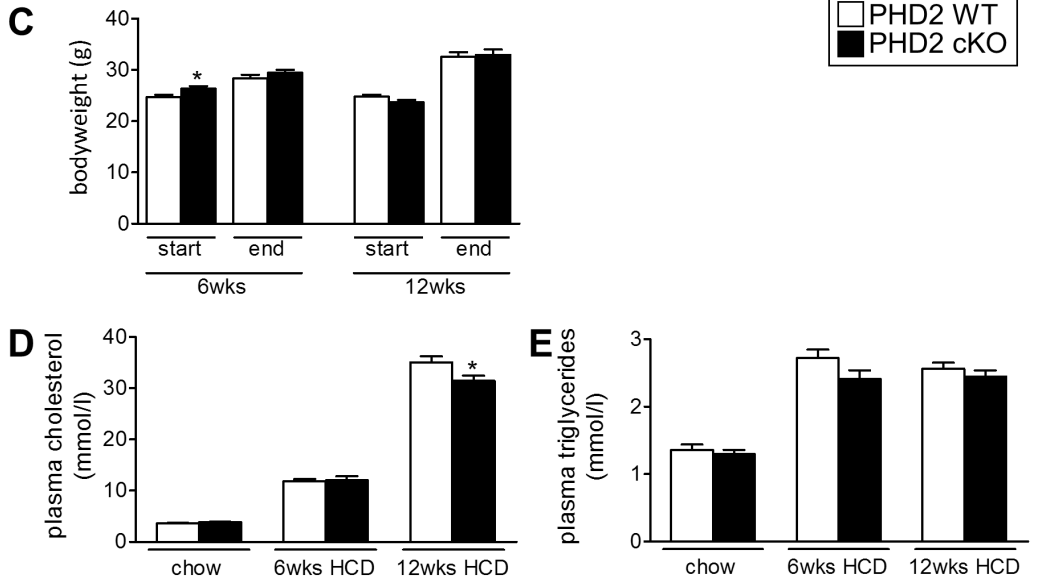

Supplemental figure 1. Increased HIF1 $\alpha$ expression in PHD2 cKO, no change in bodyweight and blood lipids

(A) HIF1 $\alpha$ and HIF2 $\alpha$ levels in macrophage lysates in vitro ( $n=4$ for both). (B) Representative image of HIF1 $\alpha$ immunoreactivity in intermediate murine plaques. (C) Bodyweight of PHD2 WT and PHD2 cKO mice given HCD for 6 or 12 weeks. (D) Plasma cholesterol and (E) plasma triglycerides of PHD2 WT vs PHD2 KO mice after 6 or 12 weeks of HCD. Mean \pm SEM *P $<0.05$. 


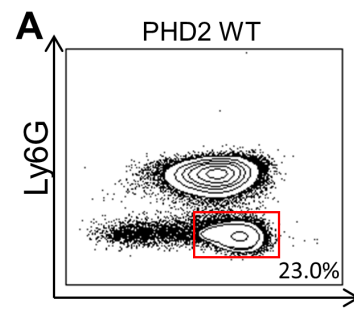

CD11b

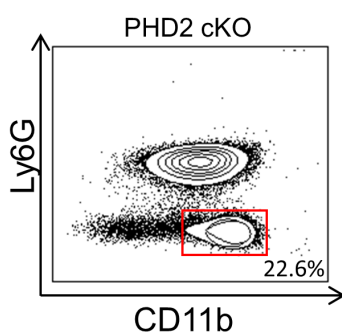

B

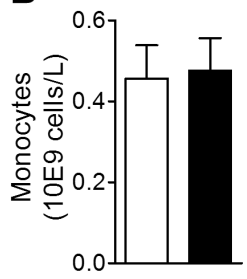

C

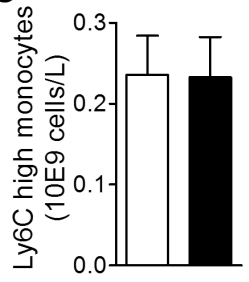

D

E

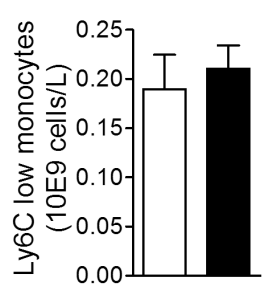

Cell type

PHD2 WT* (mean \pm SEM)

PHD2 cKO* (mean \pm SEM)

p value

\begin{tabular}{|llll}
\hline CD45 leucocytes & $3.765 \pm 0.4245$ & $3.172 \pm 0.3876$ & 0.2799 \\
\hline B cells & $0.8346 \pm 0.1053$ & $0.6441 \pm 0.09247$ & 0.1908 \\
\hline CD3 $^{+}$T cells & $0.4282 \pm 0.04103$ & $0.4037 \pm 0.04377$ & 0.6878 \\
\hline CD8 $^{+}$T cells & $0.1693 \pm 0.01794$ & $0.1667 \pm 0.1988$ & 0.9237 \\
\hline CD4 $^{+}$T cells & $0.2342 \pm 0.2103$ & $0.2167 \pm 0.02200$ & 0.5724 \\
\hline NK cells & $0.1170 \pm 0.1585$ & $0.1027 \pm 0.1022$ & 0.4582 \\
\hline NKT cells & $0.0074 \pm 0.000973$ & $0.0096 \pm 0.0018$ & 0.3316 \\
\hline Granulocytes & $1.733 \pm 0.2232$ & $1.453 \pm 0.2058$ & 0.3692 \\
\hline
\end{tabular}

*All cell values displayed as $10 \mathrm{E} 9$ cells/L

Supplemental figure 2. Unchanged immune cell subsets in PHD2 cKO mice

Gating example of flow cytometry for (A) monocytes and quantification of (B) total monocytes and (C+D) Ly6C high/low monocyte subsets after 12 weeks of diet. (E) Table of all immune cell subsets analyzed by flow cytometry. Mean \pm SEM 
Chapter 3
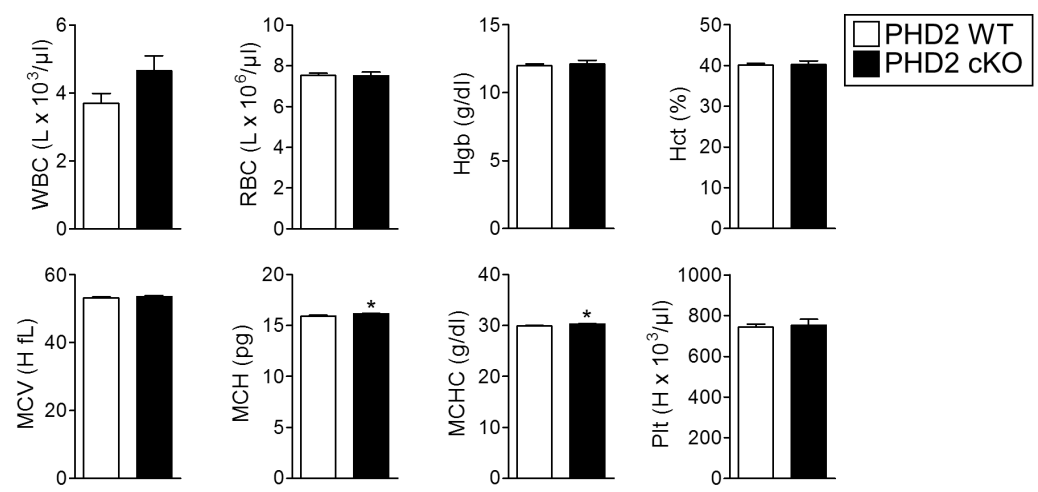

Supplemental figure 3. No difference in hematopoietic parameters in PHD2 cKO

Number of white blood cells, red blood cells, platelets, hematocrit, hemoglobin, mean cell volume, mean cell hemoglobin, mean corpuscular hemoglobin were measured in whole blood of PHD2-WT and PHD2-cKO mice. Mean \pm SEM *P $<0.05$. 

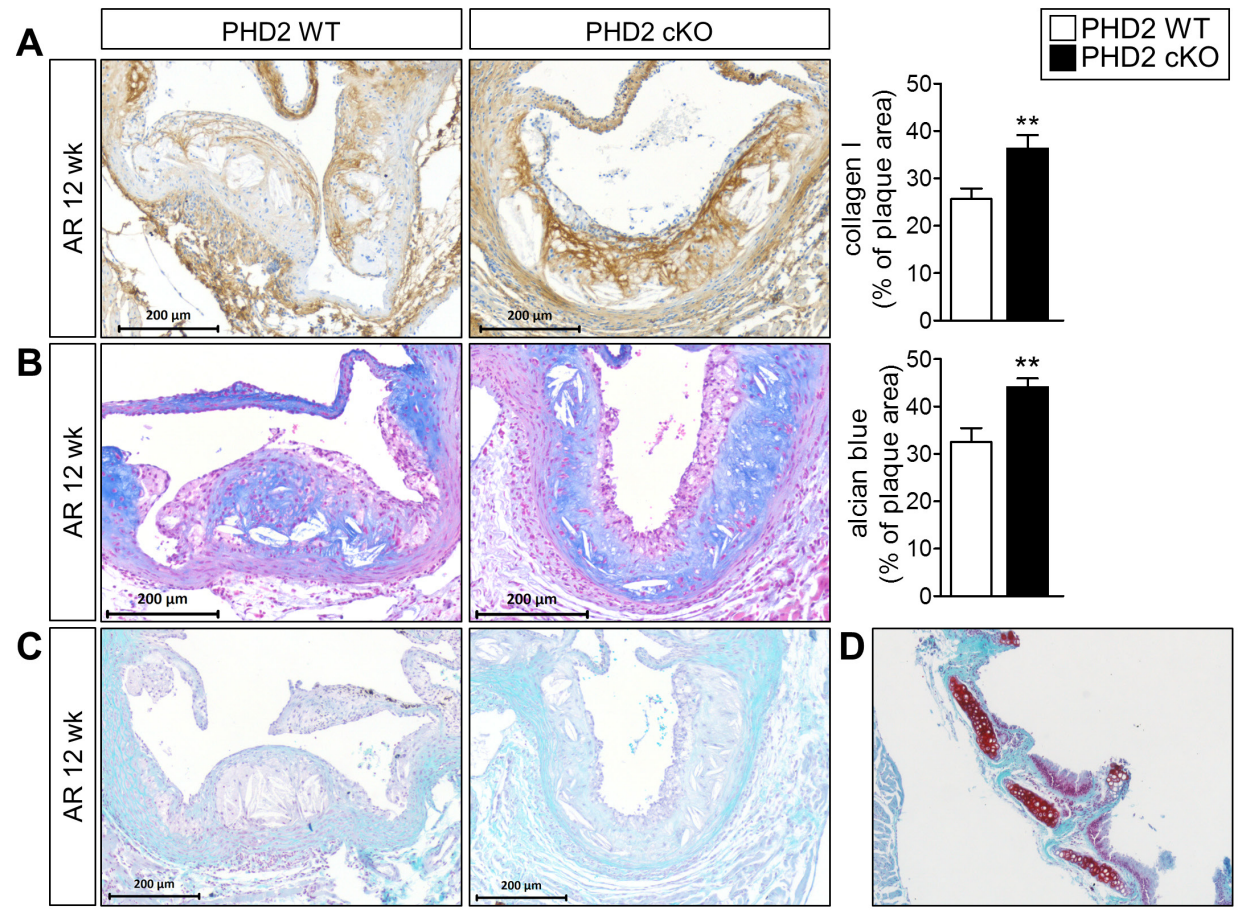

Supplemental figure 4. Increased collagen and glycosaminoglycans, but not cartilage in plaques of PHD2 cKO mice

(A) Immunohistochemistry for collagen type I in advanced plaques of PHD2 WT and PHD2 cKO mice. (B) Histological staining for glycosaminoglycans (Alcian blue) and (C) mature cartilage (Safranin O) in advanced plaques of PHD2 WT and PHD2 cKO mice, and (D) in trachea (positive control for Safranin O). Mean \pm SEM $* * P<$ 0.01 . 

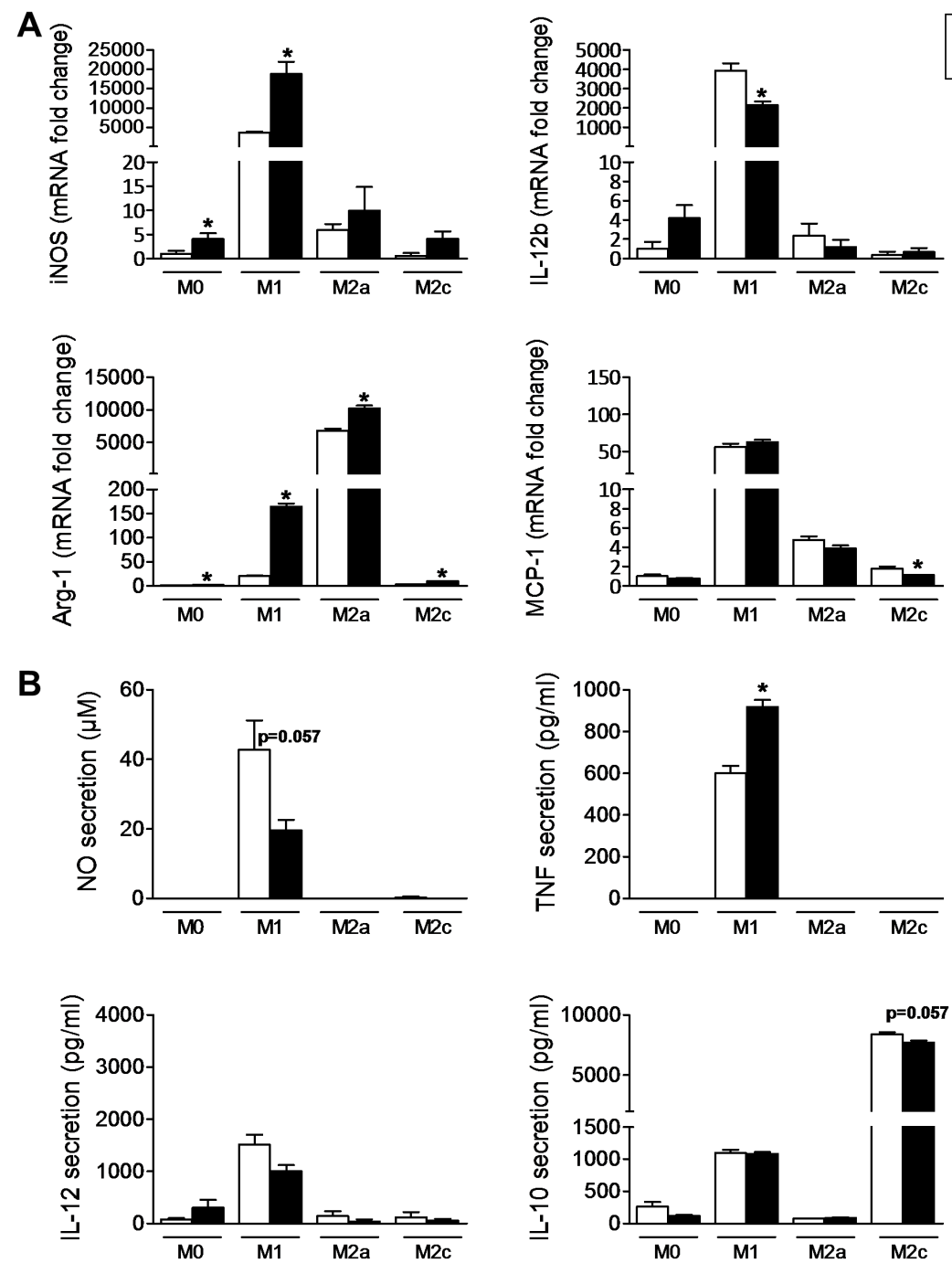

Supplemental figure 5. No distinct M1 or M2 polarization pattern in PHD2 cKO macrophages

(A) mRNA expression of macrophage polarization markers of polarized BMDM (M0=PBS, M1=LPS, M2a=IL4, $M 2 c=I L 10)$ in vitro ( $n=4$ for all). (B) Secretion of cytokine and NO by polarization markers of polarized BMDM (M0=PBS, M1=LPS, M2a=IL4, M2c=IL10) measured in conditioned medium in vitro ( $n=4$ for all). Mean \pm SEM *P < 0.05 
Myeloid PHD2 deficiency in experimental atherosclerosis | 3
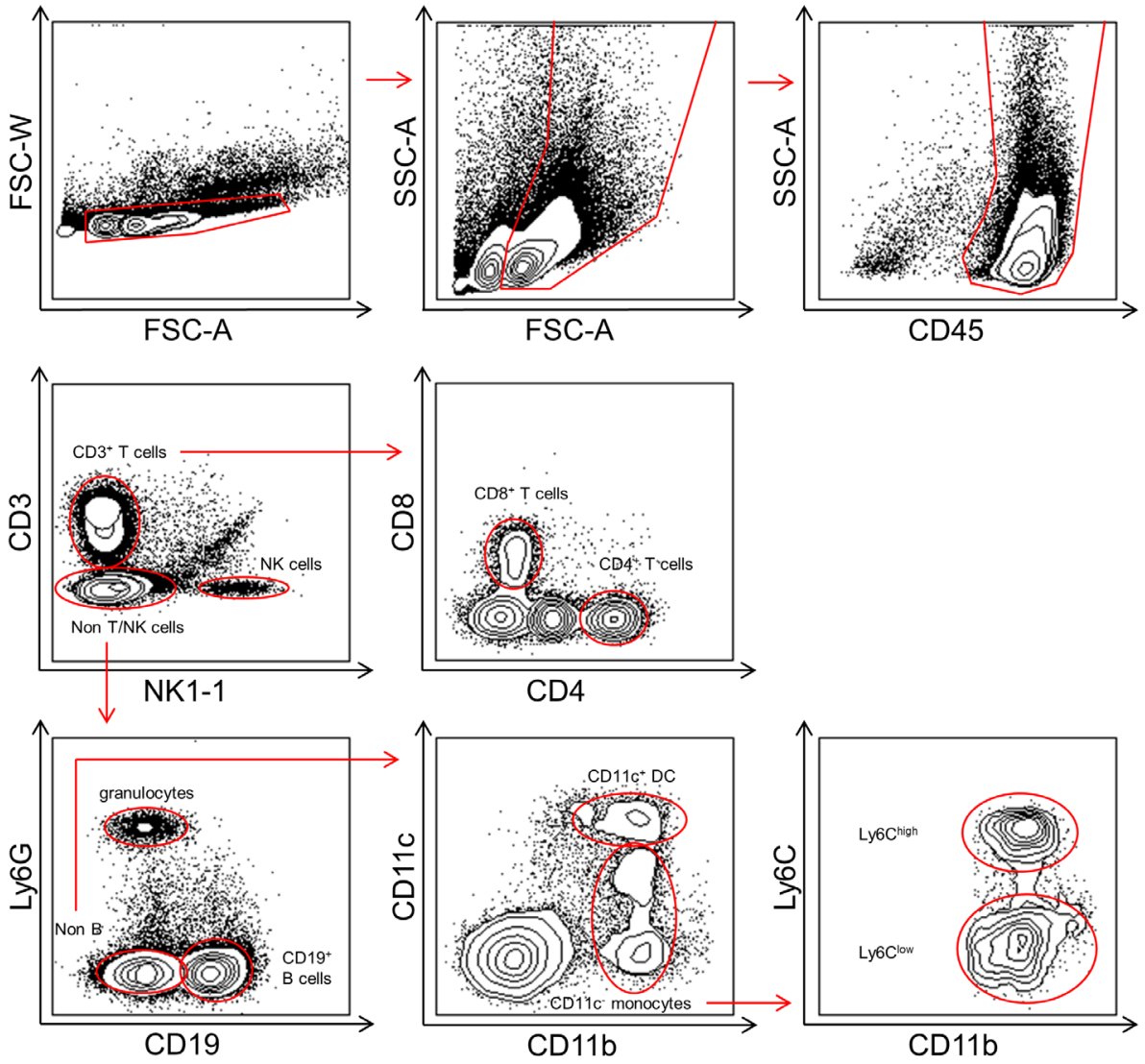

Supplemental figure 6. Gating strategy of flow cytometry 


\begin{tabular}{lll}
\hline Gene & Forward Primer & Reverse Primer \\
\hline Cyclo & CAAATGCTGGACCAAACACAA & TTCACCTTCCCAAGACCACAT \\
18S & TGCATGGCCGTTCTTAGTTG & AGTTAGCATGCCAGAGTCTCGTT \\
PHD2 & TAAACGGCCGACGAAAGC & GGGTTATCAACGTGACGGACA \\
VEGF & CAGAAGGAGGCAGAAGTCC & CTCCAGGGCTTCATCGTTA \\
Ang-1 & CGGATTTCTCTCCAGAAAC & TCCGACTTCATATTTCCACAA \\
bFGF & GCCAACCGGTACCTTGCTAT & CCCAGTTCGTTCAGTGCCA \\
P4HA1 & AAGGCTGAGCCGAGCTACA & GCCAAGCACTCTTAGATACTCTG \\
P4HA2 & AGACAGGTGTCCTCACTGTTG & GCATCTTCGTCATCGCTCCT \\
MMP3 & GCATCCCCTGATGTCCTCGTGG & TCCCCGGAGGGTGCTGACTG \\
MMP8 & TGCCTCGATGTGGAGTGCCTGA & GCCCTTGACAGCTGTGGCGT \\
MMP9 & AGAGAGGAGTCTGGGGTCTGGTTT & GAGAACACCACCGAGCTATCCACT \\
MMP12 & AATTACACTCCGGACATGAAGCGT & GGCTAGTGTACCACCTTGCCATC \\
MMP13 & ATGATGATGAAACCTGGACAAGCA & ATAGGGCTGGGTCACACTTCTCTG \\
MMP14 & ACCACAAGGACTTTGCTCTGAAG & CACCGAGCTGTGAGATTCCCTTGA \\
MMP19 & GATGAACTGGCCAGAACTGACCTT & GTCCCCGGTTGATGAGTTAGTGTC \\
MMP23 & CAAGGTTGGTGAGAGAGGGTAGGA & AGGAGTAGGTGCTGAGAACACGCT \\
MMP25 & CTCTGAGTGGCAGTGTTGGAAGA & TGATGTCAGGCTCCTGGTACTGAG \\
TIMP2 & GACTCCCCCTCAGACTCTCCCTAC & CATATTGATACCACCGCACAGGAA \\
TIMP3 & CACATCAAGGTGCCATTCAGGTAG & GTTCTCTCCTCCTCAACCCAAACA \\
\hline
\end{tabular}

Supplemental table 1. List of all used primers for quantitative PCR 


\section{Chapter 4}

\section{Deficiency of platelet derived growth factor B retention motif is associated with increased atherosclerotic plaque fibrosis and size}

Theelen TL, Marsch E, Legein B, Austen L, Janssen A, Jeurissen ML, Shiri-Sverdlov R, Gijbels MJ, Biessen EA, Betsholtz C, Daemen MJ, Sluimer JC

In preparation 


\section{ABSTRACT}

\section{Objective}

Human plaque rupture, the fatal event in atherosclerosis, has been associated with a high number of intra-plaque microvessels. More recently, also poor microvessel quality of intraplaque microvessels, defined by amongst others the absence of microvessel-surrounding pericytes has been associated with plaque rupture through leakage of erythrocytes and immune cells into the plaques, thereby increasing disease progression and rupture susceptibility. Similar leaky microvessels due to absent pericytes have been modelled in the blood-brain barrier by platelet derived growth factor B (PDGF-B) retention motif KO (PDGF-

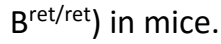

Thus, we hypothesized that microvascular pericyte loss via PDGF-B retention motif KO and subsequent increased microvascular permeability would exacerbate atherosclerosis.

\section{Approach and results}

PDGF-B ${ }^{\text {ret/ret }}$ mice were crossed with atherosclerosis-prone low density lipoprotein receptor $\mathrm{KO}\left(\mathrm{LDLr}^{-/}\right)$mice and at the age of 10 weeks fed a Western type diet $(0.25 \%$ cholesterol) for additional 10 weeks, PDGF-B ${ }^{\mathrm{WT} / \mathrm{WT}} \mathrm{LDLr}^{-/}$mice served as control. The aortic root was excised and sequential cryo-sections cut for analysis of plaque size (hematoxylin-eosin staining) and phenotype (macrophages MOMA2, microvessels CD31, smooth muscle cells aSMA, iron deposition Perls iron, plaque collagen and cap thickness Sirius Red). Arterial blood was collected during sacrifice to determine leukocyte profiles by flow cytometry and plasma lipids.

Plaque size was significantly increased in PDGF-B ${ }^{\text {ret/ret }}$ compared to control mice $(+41 \%$, $p<0.001)$, without differences in necrotic core content. The increased plaque size was independent of differences in microvessel quantity and/or quality. While plasma lipid levels were unaltered, PDGF-B ${ }^{\text {ret/ret }}$ mice featured overt leukocytosis (total leukocytes $+44 \%$, $\mathrm{p}<0.001$ ), with major increments in blood T-cells and in particular myeloid subsets. Despite increased myeloid numbers in blood, plaque macrophage content was even decreased in PDGF-B ${ }^{\text {ret/ret }}$ mice $(-50 \%, p<0.01)$. The plaque expansion in PDGF-B ${ }^{\text {ret } / \text { ret }}$ mice could be attributed to an increase in collagen content $(+25 \%, p<0.01)$, whereas smooth muscle cell contend remained unchanged.

\section{Conclusions}

PDGF-B ${ }^{\text {ret/ret }}$ resulted in plaque expansion and fibrosis independent of microvessel quantity or quality. The increase in fibrosis was unrelated to plaque smooth muscle cell content. A potentially pro-inflammatory myelocytosis in blood of PDGF-B ${ }^{\text {ret/ret }}$ mice was not reflected in plaque macrophage content. 


\section{INTRODUCTION}

Atherosclerosis still remains the major cause of death in Western society, despite the large scale cholesterol lowering treatment in $71 \%$ of cardiovascular patients (1). Atherosclerosis is a lipid driven, slowly progressing, inflammatory disease that becomes clinically relevant after plaque rupture and subsequent luminal thrombus formation. Several processes have been associated with plaque rupture, perivascular inflammation, necrotic core size, microcalcification, intra-plaque hemorrhage, plaque hypoxia and angiogenesis (2). Indeed, the formation of intra-plaque microvessels that originate from the adventitia, has been identified as major source of intra-plaque hemorrhage, i.e. the leakage of erythrocytes and immune cells into the plaque. Hence plaque and adventitial microvessels are thought to increase disease progression and severity (3). An important criterion in this association is not only microvessel quantity, but also the quality. Microvessel quality is defined by healthy endothelial cell morphology, intact endothelial junctions, an intact basement membrane and especially the presence of microvessel surrounding pericytes. Microvessels in ruptured human coronary plaques generally display with endothelial abnormalities and the absence of stabilizing, and supportive pericytes (4). Pericytes are recruited to the endothelium by platelet derived growth factor B (PDGF-B), which is secreted by healthy endothelial cells. PDGF-B is anchored in the extracellular matrix via its retention motif, forming a growth factor gradient, which guides pericytes towards the endothelial cells of the developing vessel (5). Disruption of this gradient by deletion of the retention motif was seen to induce pericyte loss, and subsequently to increase microvascular leakage of the blood-brain barrier (6). Vice versa, absence of pericyte coverage and vessel dysfunction, can be restored by overexpressing PGDF-B (7).

Besides its role in angiogenesis, PDGF-B may have effects on other cell types involved in atherogenesis. For instance, PDGF-B stimulates smooth muscle cell proliferation (8) and extracellular matrix formation (9). Also, immune cells have been shown to express PDGF-B (10) and hematopoietic KO of PDGF-B resulted in a pro-inflammatory phenotype as it increased numbers of activated CD4 T cell in blood and monocyte accumulation in plaques of $\mathrm{ApoE}^{-/}$mice (11).

Thus, we hypothesized that microvascular pericyte loss via PDGF-B retention motif $\mathrm{KO}$ and subsequent increased microvascular permeability would exacerbate atherosclerosis. 


\section{MATERIALS AND METHODS}

\section{Experimental animals}

All animal experiments were conducted according to Dutch governmental guidelines and approved by the Dutch regulatory authorities. PDGF-B retention motif knockout (PDGF$B^{\text {ret/ret }} \mathrm{KO}$ ) mice with XlacZ knockin as pericyte marker were provided by Betzholz (6) and crossed with low density lipoprotein receptor ( $\left.\mathrm{LLLr}^{-/}\right) \mathrm{KO}$ mice from an in house breeding colony. All mice were crossed back on a C57BI6 background at least nine times. PDGF-B retention motif ${ }^{\mathrm{wt} / \mathrm{wt}} \mathrm{LDLr}^{-/-}$mice served as control in all experiments (referred to as control). For all experiments male mice were used (control $n=19$, PDGF- $B^{\text {ret } / r e t} n=10$ ). Animals were housed in the laboratory animal facility of Maastricht University under standard conditions. Food and water were provided ad libitum during the entire experiment.

\section{Atherosclerosis induction and plaque size measurement}

At the age of 10 weeks, mice were fed a Western type diet containing: $0.25 \%$ cholesterol (Special Diet Services, \#824171, 15\% cocoa butter, 10\% maize starch, 20\% casein, 40.5\% sucrose, $5.95 \%$ cellulose) for 10 weeks to induce atherosclerosis. All mice were euthanized by an intraperitoneal pentobarbital injection $(100 \mathrm{mg} / \mathrm{kg})$ and heart puncture of the right ventricle. Whole blood was collected to analyze hematopoietic parameters, immune cell subsets (flow cytometry), and plasma cholesterol and plasma triglyceride content. Mice were then perfused with PBS-sodium nitroprusside dehydrate. The aortic root was dissected and immediately embedded in OCT compound. Serial cryo-sections $(5 \mu \mathrm{m})$ from the OCT embedded aortic roots were cut and HE stained to quantify plaque size and necrotic core content in four representative sections of the aortic root using computerized morphometry (Leica QWin V3, Cambridge, UK). The necrotic core was defined as acellular and anuclear plaque areas, rich in cholesterol clefts.

\section{Histology and immunohistochemistry}

Atherosclerotic plaques were analyzed for collagen (Sirius red area/plaque area, Polyscience 09400), iron (Perls Prussian blue), macrophage content (MOMA-2 area/plaque area, produced by M.L.J.), smooth muscle cell content ( $\alpha \mathrm{SMA}^{+}$area/ plaque area, $\alpha \mathrm{SMA}$ Sigma F3777) and adventitial microvessel density (number of CD31 ${ }^{+}$ microvessels/adventitial area, BD Cat. No. 550274). Mean fibrous cap thickness was determined in the aortic root as described in (12) using ImageJ software.

\section{Blood count and flow cytometry}

White blood cells, red blood cells and platelets counts, hematocrit, hemoglobin, mean cell volume, mean cell hemoglobin, and mean corpuscular hemoglobin were measured using a Coulter Counter (Beckman Coulter, Woerden, NL). Flow cytometry was performed to quantify absolute immune cell subsets in blood (TrueCount BD, Cat No 340334). Erythrocytes were lysed before antibody labelling. Combinations of the following antibodies were used to characterize leucocytes (CD45 Biolegend clone 30-F11), B-cells (B220 BD clone RA3-6B2), T-helper cells (CD3 eBioScience clone 145-2c11/CD4 BD clone GK1.5), cytotoxic T-cells (CD3/CD8 eBioScience clone 53-6.7), NK cells (CD8/NK1.1 BD clone PK136), monocyte subsets (Ly6C Miltenyi clone 1G7.G10/CD11b BD clone M1/70) and granulocytes (Ly6G BD clone 1A8/CD11b). Gating was performed as described previously (13). 


\section{Plasma lipid measurements}

Plasma samples were obtained by centrifugation of whole blood drawn from the heart, and stored at $-80^{\circ} \mathrm{C}$ until further use. Standard enzymatic techniques were used to assess plasma cholesterol (CHOD-PAP method - Cholesterol FS Ecoline product no. 113009990314, DiaSys, Holzheim, GE) and plasma triglycerides (FS5' Ecoline REF 157609990314; DiaSys Diagnostic Systems $\mathrm{GmbH}$, Holzheim, Germany) automated on the Cobas Fara centrifugal analyzer (Roche).

\section{Statistical analysis}

All data are presented as mean \pm SEM. Shapiro-Wilk test for normal distribution was performed, followed by a Grubbs' outlier test. Finally, groups were compared with student's t-test or Mann-Whitney rank-sum test. (GraphPad Prism4, La Jolla, CA, USA). A p-value of $p<0.05$ was considered significant $\left(* p<0.05,{ }^{* *} p<0.01, * * * p<0.001\right)$. 


\section{RESULTS}

\section{Increased plaque size after PDGF-B retention motif KO}

To investigate the effect of PDGF-B retention motif $K O$ on atherosclerosis, mice were fed a Western type diet for 10 weeks. Weight gain after 10 weeks of Western type diet was less in PDGF-B ${ }^{\text {ret/ret }}$ mice (Figure $1 \mathrm{~A}$ ). This effect was independent of changes in plasma lipids, as both plasma cholesterol and triglyceride levels were not affected by the PDGF-B ${ }^{\text {ret/ret }} \mathrm{KO}$ (Figure 1B). As hypothesized, plaques of PDGF-B ${ }^{\text {ret/ret }}$ mice displayed a significantly increase in size of $41 \%$ compared to control (Figure $2 A, B$ ). The increase in plaque size was not accompanied by an increase in necrotic core content (Figure $2 \mathrm{C}$ ).

A

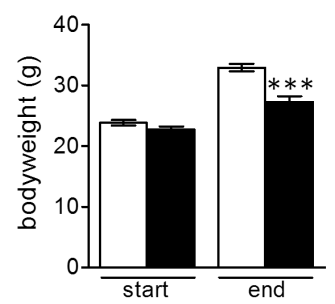

B

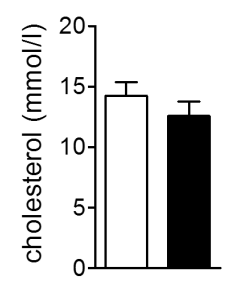

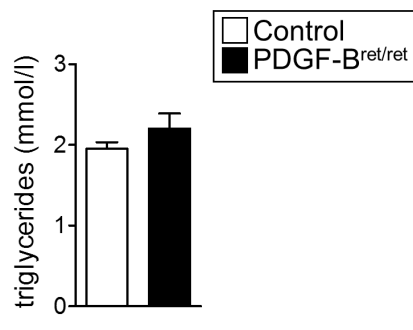

Figure 1. PDGF-B $\mathrm{B}^{\text {ret/ret }}$ mice have reduced diet-induced weight gain with similar plasma lipid levels (A) Bodyweight of control and PDGF-B ret/ret $^{2}$ the beginning and end of Western type diet feeding. (B) Plasma cholesterol levels (left graph) and plasma triglyceride levels (right graph) diets after 10 weeks of Western type diet. Mean \pm SEM $* * * p<0.001$
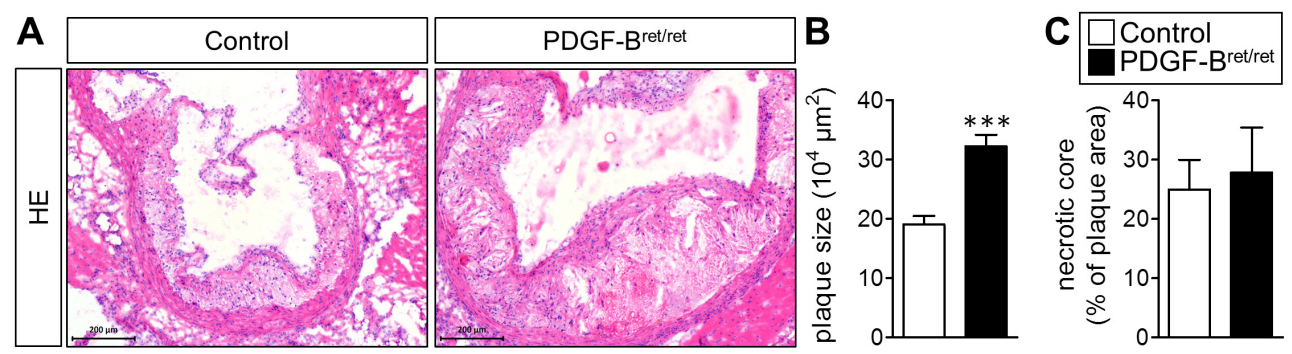

Figure 2. PDGF-B retention motif KO increased plaque size, but not necrotic core

(A) Representative images and (B) Quantification of plaque size, and (C) necrotic core percentage on hematoxylin eosin stained plaques of the aortic root after 10 weeks of high cholesterol diet. Mean \pm SEM $* * * p<0.001$ 


\section{Increased plaque size unrelated microvessel quality}

PDGF-B ${ }^{\text {ret/ret }} \mathrm{KO}$ has been shown to increase microvessel leakage at the blood-brain barrier (5). However, no CD31 positive microvessels could be detected in plaque of WT or of PDGF$B^{\text {ret/ret }} \mathrm{KO}$ (Figure $3 \mathrm{~A}$ ). Due to the lack of intra-plaque vessels, we assessed adventitial plaque vessel quantity (CD31, Figure 3B) as a surrogate parameter. However, also adventitial vessel quantity did not differ between genotypes. In line, no difference in intra-plaque iron residues, potentially originating from lysed erythrocytes after hemorrhage, could be found in PDGF-B ${ }^{\text {ret/ret }}$ compared to WT mice (Figure 3C).
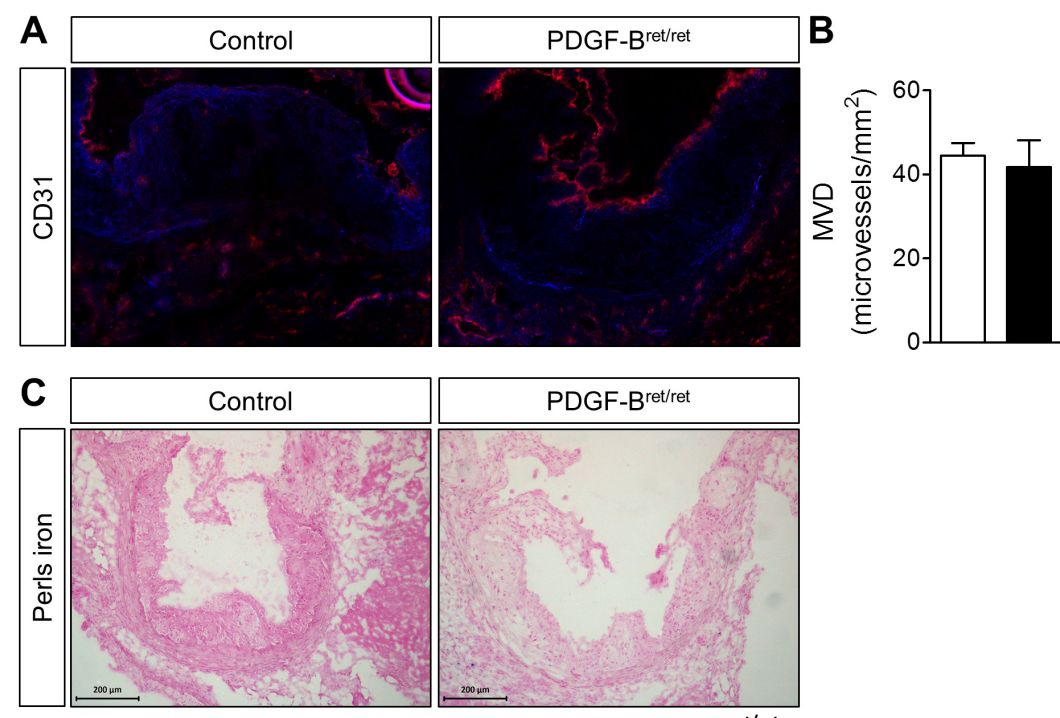

Figure 3. Unaltered microvessel quantity or quality by PDGF-B ${ }^{\text {ret/ret }}$

(A) Representative pictures and (B) quantification of CD31+ microvessels (red) in the adventitia. (C) Representative pictures of Perls iron staining (both negative for Perls iron). Mean \pm SEM 


\section{PDGF-B ${ }^{\text {ret/ret }}$ increased leukocytes numbers in blood}

Atherosclerosis is an inflammatory disease and immune cell numbers can causally influence atherogenesis. Therefore, blood counts were first analyzed by coulter counter showing marked increases in blood leukocytes of PDGF-B ${ }^{\text {ret/ret }}$ mice (Figure 4). Apart from B-cells, all of the analyzed immune cell subsets were increased in PDGF-B ${ }^{\text {ret } / \text { ret }}$ mice (Figure 5). Especially the observed monocytosis and granulocytosis point to a pro-inflammatory immune cell status of the PDGF-B ${ }^{\text {ret/ret }}$ KO mice.
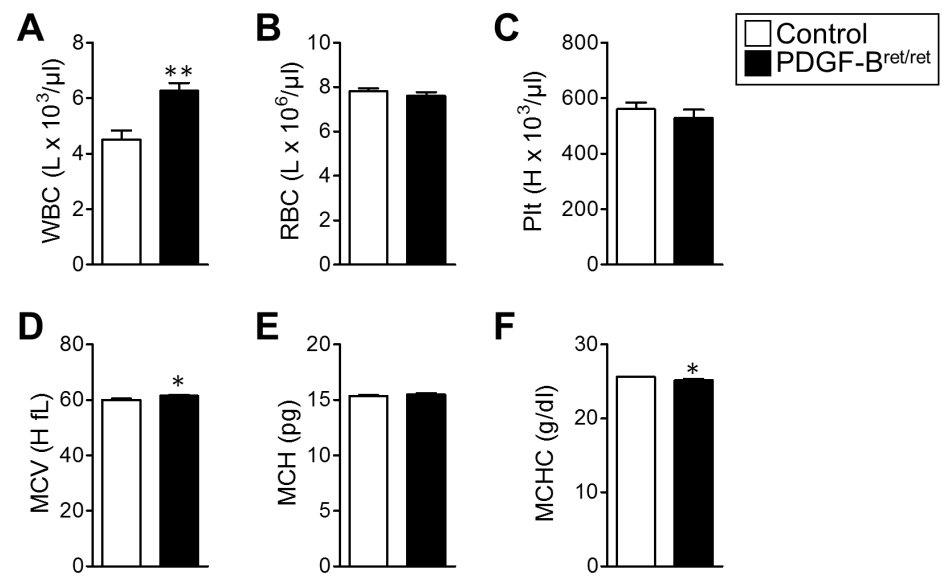

F
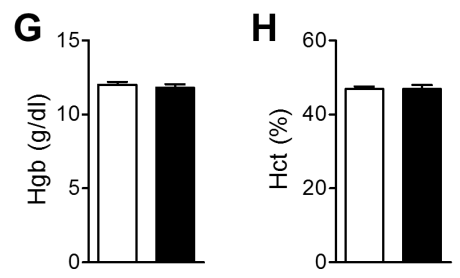

Figure 4. Increased circulating white blood cells in PDGF-B ${ }^{\text {ret/ret }}$ mice

Number of (A) white blood cells, (B) red blood cells, (C) platelets, (D) mean cell volume, (E) mean cell hemoglobin, (F) mean corpuscular hemoglobin, $(\mathbf{G})$ hemoglobin and $(\mathrm{H})$ hematocrit in whole blood of control and PDGF-B ${ }^{\text {ret/ret }}$ mice. Mean \pm SEM $* p<0.05, * * p<0.01$ 


\section{No increase in plaque macrophages}

Atherosclerotic plaque macrophages are thought to originate from extravasated circulating monocytes or by clonal expansion (14). Although overall blood monocytes including the tissue homing Ly6C $\mathrm{C}^{\text {high }}$ monocyte species were elevated (Figure 5), and although PDGF-B was previously reported to stimulate macrophage proliferation in vitro (15), the percentage of MOMA2-positive macrophage plaque content was even decreased in PDGF-B ${ }^{\text {ret } / r e t} \mathrm{KO}$ mice (Figure 6A,B).

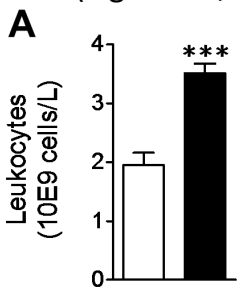

E

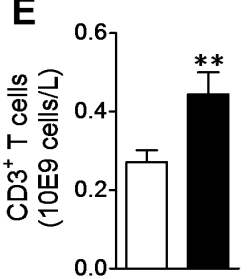

H

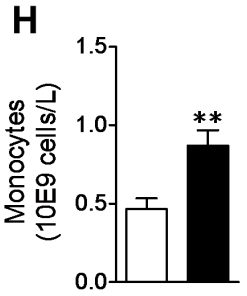

B

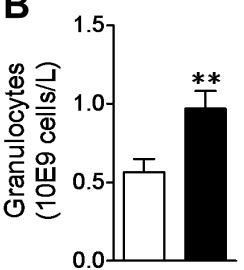

$\mathbf{F}$
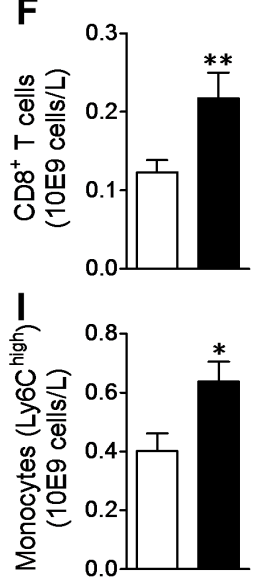

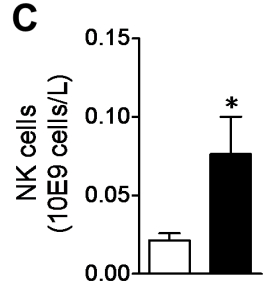

G
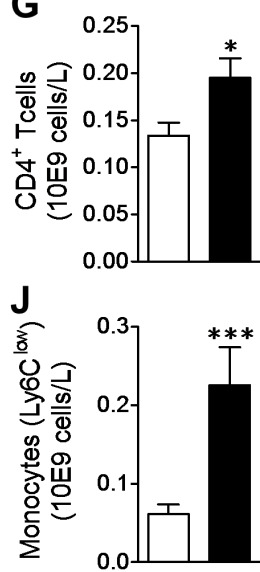
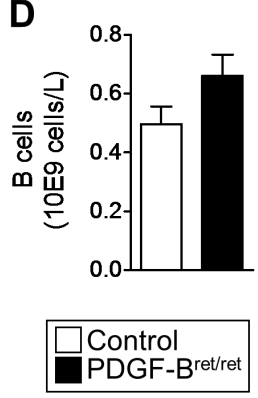

Figure 5. PDGF-B ${ }^{\text {ret/ret }}$ mice display a pro-inflammatory leukocyte phenotype in circulating blood Flow cytometry for (A) total leukocytes, (B) granulocytes, (C) NK cells, (D) B cells, (E) total T cells, (F) cytotoxic T cells, (G) T helper cells, (H) total monocytes, (I) tissue homing monocytes (Ly6Chigh) and (J) patrolling monocytes (Ly6Clow). Mean \pm SEM *p $<0.05, * * p<0.01, * * * p<0.001$ 


\section{Increased collagen content with no change in smooth muscle cell content}

PDGF-B is an important factor for smooth muscle cell function and proliferation (16). Therefore, plaques were analyzed for smooth muscle cell presence. No increase in the percentage of plaque smooth muscle cells could be found in plaques of PDGF-B ${ }^{\text {ret/ret }}$ mice (Figure 6C,D). In contrast, collagen content (Figure 6E,F) and cap thickness (Figure 6G) were

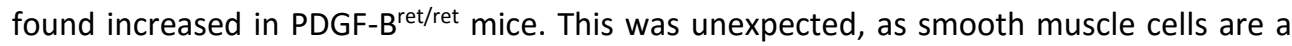
major source of collagen in atherosclerosis (16).
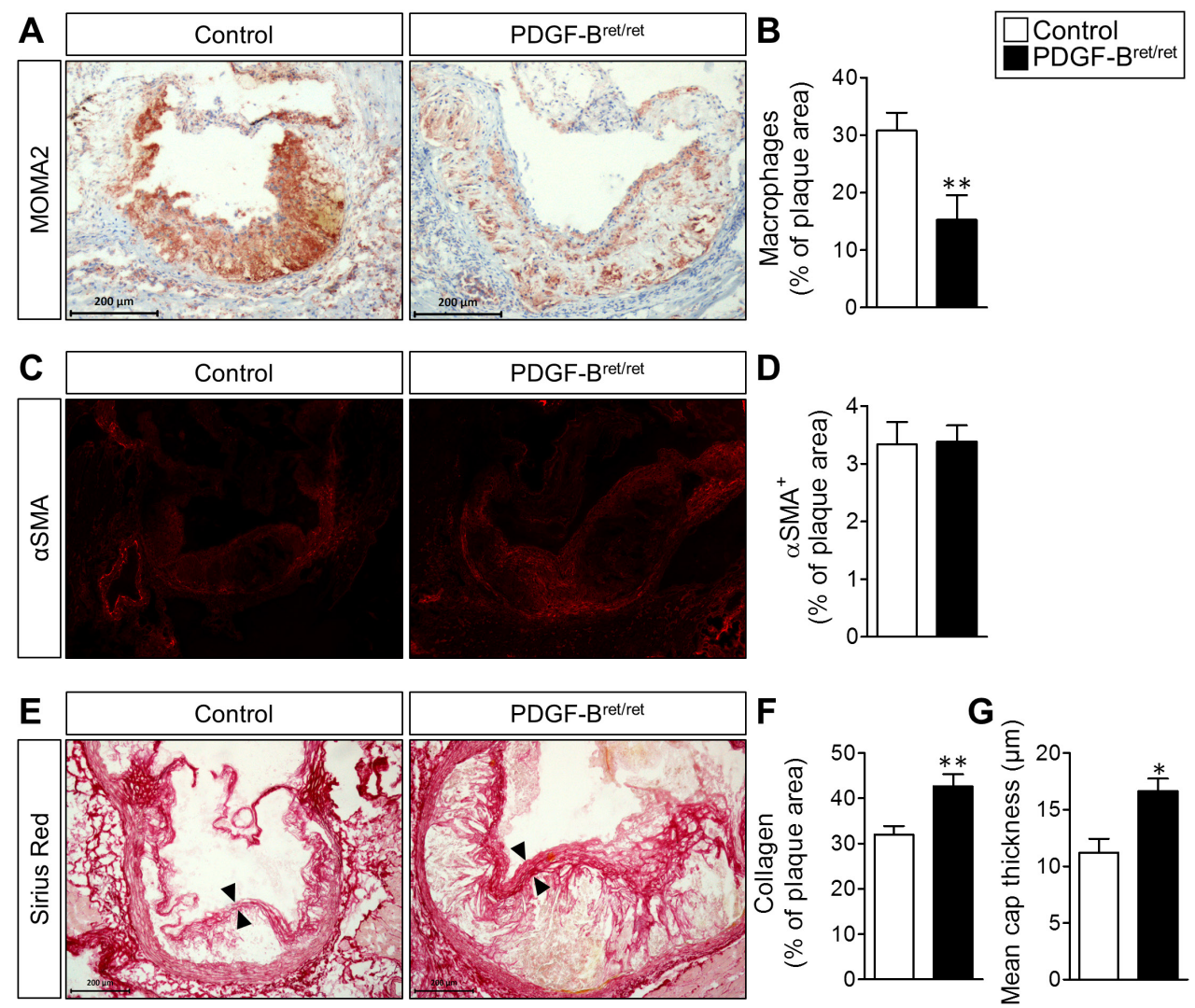

Figure 6. Increased plaque size in PDGF-B ${ }^{\text {ret/ret }}$ mice caused by increased collagen content rather than macrophages and smooth muscle cells

(A) Representative pictures and (B) quantification of MOMA-2 stained macrophages and (C+D) $\alpha$ SMA stained smooth muscle cells (red) in plaques of the aortic root. (E) Representative pictures and (F) quantification of Sirius red stained plaque collagen and $(\mathbf{G})$ mean cap thickness. Mean $\pm S E M * p<0.05, * * p<0.01$ 


\section{DISCUSSION}

Disruption of PDGF-B's ability to anchor to extracellular matrix, by deleting its retention motif, led to increased plaque size in atherosclerosis-prone mice. Surprisingly, this did neither affect plaque or adventitia microvessel number nor leakage, as assessed by iron deposition. The increase in plaque size was accompanied by an increase in leukocyte counts in blood and in particular of major myeloid subsets relevant to atherosclerosis. However, the increased circulating monocyte and granulocyte presence did not translate into enhanced plaque macrophage content. Rather, we observed clear profibrotic effects: collagen content and cap thickness increased and likely accounted for the plaque expansion in PDGF-B ${ }^{\text {ret/ret }}$ mice. Interestingly, the increase in fibrosis was independent of plaque smooth muscle cell content. PDGF-B ${ }^{\text {ret/ret }}$ reduced weight gain after Western type diet feeding. However, the difference in bodyweight did not reflect a diminished food intake and related plasma lipid profiles, as cholesterol and triglyceride levels were similar.

\section{No effect of PDGF-B ${ }^{\text {ret/ret }}$ on adventitial microvessel quantity or quality}

It was reported previously that PDGF-B ${ }^{\text {ret/ret }}$ reduced microvessel density in the retina (5), suggesting reduced angiogenesis in PDGF-B ${ }^{\text {ret/ret }} \mathrm{KO}$ mice. In line no intra-plaque vessels were found in PDGF-B ${ }^{\text {ret/ret }}$ mice, however, also no intra-plaque vessels could be found in control mice. In addition, no difference in adventitial microvessel numbers was observed. The pericyte coverage of adventitial microvessels, thus microvessel quality, remains to be elucidated in the current model using pericyte markers such as the XlacZ transgene and/or NG2. However, the absence of iron deposition in the plaque hints to similar microvessel leakage as intra-plaque and adventitial hemorrhage is absent in both control and PDGF$\mathrm{B}^{\text {ret/ret }}$ mice.

\section{Despite increased leukocytes in blood, less macrophages in PDGF-B ${ }^{\text {ret/ret }}$ plaques}

A prominent feature of PDGF-B ${ }^{\text {ret/ret }}$ mice was the leukocytosis, with expansion of almost all subsets in circulation. This effect has not been reported earlier, but a possible explanation for the elevated blood immune cell counts could be differences in hematopoiesis in PDGF$\mathrm{B}^{\text {ret/ret }}$ mice. Culture of bone marrow cells with PDGF-B led to activation of primitive hematopoietic precursors via the upregulation of interleukin 1 in marrow macrophages (18). However in vivo proof of this concept is absent. In contrast to the in vitro observations, bone marrow reconstitution of lethally irradiated mice with fetal PDGF-B KO liver cells showed no effect on hematopoiesis (19). In this model, PDGF-B is completely absent, while in our model PDGFB is not anchored into the extracellular matrix. This may result in redistribution of extracellular PDGFB from tissue to circulation. Besides possible effects on hematopoiesis, PDGF-B may act on circulating immune cells. Myeloid PDGF-B deficiency was reported to increase $T$ cell number, resulted in pro-inflammatory $T$ cell skewing and led to increased intimal monocyte recruitment (20). However, the elevated leukocyte counts in blood did not correlate with macrophage content within the plaques, as the macrophage content was even found to be decreased. This may be explained by the recent finding that plaque macrophage numbers in advanced plaques rather depend on local proliferation, instead of monocyte recruitment from the circulation (21). However, PDGF-B stimulation was reported to induce macrophage proliferation in vitro (15) suggesting lower PDGF-B levels within the analyzed plaques of PDGF-B ${ }^{\text {ret/ret }}$ mice. The plaque PDGF-B levels remain 
elucidated in the current study, but the lack of the matrix-retention motif may indeed result in lower plaque PDGF-B. Also, the effect of increased immune cells counts on other plaque immune cells such as $T$ cells and granulocytes remains to be investigated in the current model.

\section{PDGF-B pro-atherogenic via effects on smooth muscle cells}

There are strong indications that PDGF-B may play a detrimental role in atherosclerosis, as local overexpression (22) or PDGF-B infusion (23) resulted in larger plaques. In both studies, smooth muscle cell proliferation could be identified as causal mechanism for the expansion. Since in the present study no difference in smooth muscle cell content was found, the altered PDGF-B distribution after retention motif $\mathrm{KO}$ might rather act on smooth muscle cell functionality than proliferation. The possible effect on smooth muscle cell functionality is supported by a previous finding that PDGF-B stimulates collagen production in smooth muscle cell in vitro (24) and in vivo (25). This is in line with the current observation of increased total collagen and cap thickness in PGDF-B ${ }^{\text {ret/ret }}$ mice. Thus, elevated serum PDGF$B$ levels in PDGF-B ${ }^{\text {ret/ret }}$ would partially explain the current findings. As PDGF-B after retention motif $K O$ cannot anchor in the extracellular matrix, serum levels may be enhanced by increased diffusion. To explain our findings, an altered distribution of PDGF-B after retention motif $\mathrm{KO}$ in plaques and plasma seems likely, but remains to be proven.

\section{Dual role of PDGF-B in atherosclerosis}

PDGF-B expression is enhanced at predilection sites of atherosclerosis exposed to turbulent flow and low shear stresses (26). PDGF-B is also strongly expressed in atherosclerotic plaques, compared to non-diseased vessels (27). In addition, high PDGF-B plasma levels were associated with hypercholesterolemia (28), stenosis (29) and symptomatic patients (30). Moreover, inhibition of PDGF-B receptor by either a kinase inhibitor (31) or a blocking antibody (32) inhibited atherosclerosis in atherosclerosis-prone $\mathrm{ApoE}^{-/-}$mice. Besides the aforementioned pro-atherogenic effects of PDGF-B and the results of the current study, also anti-atherogenic properties have been reported. Hematopoietic PDGF-B KO resulted in a pro-inflammatory phenotype in atherosclerosis (11) and PDGF-B may thus have antiinflammatory properties. This is supported by the finding that stimulation of isolated NK cells with PDGF-B results in reduction of cytotoxicity (33). Finally, PDGF-B inhibition was not able to prevent stent restenosis in patients with pre-existing atherosclerosis (34), but did not accelerate the disease either. Thus PDGF-B inhibition or overexpression does not represent an ideal target for atherosclerosis treatment, suggesting the further investigation of downstream pathways to isolate beneficial and detrimental effects of PDGF-B in different stages of atherogenesis.

\section{Conclusion}

Retention motif KO of PDGF-B resulted in a pro-inflammatory immune cell phenotype in the blood, while plaque macrophage content even decreased. Rather, the plaque expansion in PDGF-B ${ }^{\text {ret/ret }}$ mice could be attributed to an increase in collagen content, whereas smooth muscle cell content remained unchanged. 


\section{AKNOWLEDGEMENTS}

The authors thank the staff of the laboratory animal center Maastricht for assistance with mouse breeding and housing. Research was supported by a VENI fellowship of the Netherlands Organization of Scientific research (to J.C.S. 016.116.017) and a PhD-student fellowship from the Cardiovascular Research Institute Maastricht (to T.T.). 


\section{REFERENCES}

1. Gu Q, Paulose-Ram R, Burt VL, Kit BK. Prescription cholesterol-lowering medication use in adults aged 40 and over: United States, 2003-2012. NCHS Data Brief. 2014 Dec;(177):1-8.

2. Bentzon JF, Otsuka F, Virmani R, Falk E. Mechanisms of plaque formation and rupture. Circ Res. 2014 Jun 6;114(12):1852-66.

3. Kolodgie FD, Gold HK, Burke AP, Fowler DR, Kruth HS, Weber DK, et al. Intraplaque hemorrhage and progression of coronary atheroma. N Engl J Med. 2003 Dec 11;349(24):2316-25.

4. Sluimer JC, Kolodgie FD, Bijnens APJJ, Maxfield K, Pacheco E, Kutys B, et al. Thin-walled microvessels in human coronary atherosclerotic plaques show incomplete endothelial junctions relevance of compromised structural integrity for intraplaque microvascular leakage. J Am Coll Cardiol. 2009 Apr 28;53(17):1517-27.

5. Lindblom P, Gerhardt H, Liebner S, Abramsson A, Enge M, Hellstrom M, et al. Endothelial PDGF-B retention is required for proper investment of pericytes in the microvessel wall. Genes Dev. 2003 Aug 1;17(15):1835-40.

6. Armulik A, Genové G, Mäe M, Nisancioglu MH, Wallgard E, Niaudet $C$, et al. Pericytes regulate the blood-brain barrier. Nature. 2010 Nov 25;468(7323):557-61.

7. Abramsson A, Lindblom P, Betsholtz C. Endothelial and nonendothelial sources of PDGF-B regulate pericyte recruitment and influence vascular pattern formation in tumors. J Clin Invest. 2003 Oct;112(8):1142-51.

8. Deguchi J, Namba T, Hamada H, Nakaoka T, Abe J, Sato O, et al. Targeting endogenous platelet-derived growth factor B-chain by adenovirus-mediated gene transfer potently inhibits in vivo smooth muscle proliferation after arterial injury. Gene Ther. 1999 Jun;6(6):956-65.

9. Cattaruzza M, Nogoy N, Wojtowicz A, Hecker M. Zinc finger motif-1 antagonizes PDGF-BB-induced growth and dedifferentiation of vascular smooth muscle cells. FASEB J. 2012 Dec;26(12):4864-75.

10. Billett MA, Adbeish IS, Alrokayan SA, Bennett AJ, Marenah CB, White DA. Increased expression of genes for platelet-derived growth factor in circulating mononuclear cells of hypercholesterolemic patients. Arterioscler Thromb Vasc Biol. 1996 Mar;16(3):399-406.

11. Tang J, Kozaki K, Farr AG, Martin PJ, Lindahl P, Betsholtz C, et al. The absence of platelet-derived growth factor- $B$ in circulating cells promotes immune and inflammatory responses in atherosclerosisprone ApoE-/- mice. Am J Pathol. American Society for Investigative Pathology; 2005 Sep;167(3):90112.

12. Seimon TA, Wang Y, Han S, Senokuchi T, Schrijvers DM, Kuriakose G, et al. Macrophage deficiency of p38alpha MAPK promotes apoptosis and plaque necrosis in advanced atherosclerotic lesions in mice. J Clin Invest. 2009 Apr;119(4):886-98.

13. Theelen et al. Myeloid Prolyl Hydroxylase Domain-Containing Protein 2 Deficiency Leads to Larger but more Stable Atherosclerotic Plaques in Mice. Unpublished. 2016;

14. Robbins CS, Hilgendorf I, Weber GF, Theurl I, Iwamoto Y, Figueiredo J-L, et al. Local proliferation dominates lesional macrophage accumulation in atherosclerosis. Nat Med. 2013 Sep;19(9):1166-72.

15. Inaba T, Shimano H, Gotoda T, Harada K, Shimada M, Ohsuga J, et al. Expression of platelet-derived growth factor beta receptor on human monocyte-derived macrophages and effects of platelet-derived growth factor BB dimer on the cellular function. J Biol Chem. 1993 Nov 15;268(32):24353-60.

16. Hellström M, Kalén M, Lindahl P, Abramsson A, Betsholtz C. Role of PDGF-B and PDGFR-beta in recruitment of vascular smooth muscle cells and pericytes during embryonic blood vessel formation in 
the mouse. Development. 1999 Jun;126(14):3047-55.

17. Bentzon JF, Falk E. Atherosclerotic lesions in mouse and man: is it the same disease? Curr Opin Lipidol. 2010 Oct;21(5):434-40.

18. Yan XQ, Brady G, Iscove NN. Platelet-derived growth factor (PDGF) activates primitive hematopoietic precursors (pre-CFCmulti) by up-regulating IL-1 in PDGF receptor-expressing macrophages. J Immunol. 1993 Mar 15;150(6):2440-8.

19. Kaminski WE, LindahI P, Lin NL, Broudy VC, Crosby JR, Hellström M, et al. Basis of hematopoietic defects in platelet-derived growth factor (PDGF)-B and PDGF beta-receptor null mice. Blood. $2001 \mathrm{Apr}$ 1;97(7):1990-8.

20. Kozaki K, Kaminski WE, Tang J, Hollenbach S, Lindahl P, Sullivan C, et al. Blockade of platelet-derived growth factor or its receptors transiently delays but does not prevent fibrous cap formation in ApoE null mice. Am J Pathol. American Society for Investigative Pathology; 2002 Oct;161(4):1395-407.

21. Ye Y, Calcagno C, Binderup T, Courties G, Keliher EJ, Wojtkiewicz GR, et al. Imaging Macrophage and Hematopoietic Progenitor Proliferation in Atherosclerosis. Circ Res. 2015 Oct 23;117(10):835-45.

22. Nabel EG, Yang Z, Liptay S, San H, Gordon D, Haudenschild CC, et al. Recombinant platelet-derived growth factor $\mathrm{B}$ gene expression in porcine arteries induce intimal hyperplasia in vivo. J Clin Invest. 1993 Apr;91(4):1822-9.

23. Jawien A, Bowen-Pope DF, Lindner V, Schwartz SM, Clowes AW. Platelet-derived growth factor promotes smooth muscle migration and intimal thickening in a rat model of balloon angioplasty. J Clin Invest. 1992 Feb;89(2):507-11.

24. Amento EP, Ehsani N, Palmer H, Libby P. Cytokines and growth factors positively and negatively regulate interstitial collagen gene expression in human vascular smooth muscle cells. Arterioscler Thromb. 1991 Sep 1;11(5):1223-30.

25. Pompili VJ, Gordon D, San H, Yang Z, Muller DW, Nabel GJ, et al. Expression and function of a recombinant PDGF B gene in porcine arteries. Arterioscler Thromb Vasc Biol. 1995 Dec;15(12):2254-64.

26. Mondy JS, Lindner V, Miyashiro JK, Berk BC, Dean RH, Geary RL. Platelet-derived growth factor ligand and receptor expression in response to altered blood flow in vivo. Circ Res. 1997 Sep;81(3):320-7.

27. Raines EW. PDGF and cardiovascular disease. Cytokine Growth Factor Rev. 2004 Aug;15(4):237-54.

28. Bath PM, Martin JF. Serum platelet-derived growth factor and endothelin concentrations in human hypercholesterolaemia. J Intern Med. 1991 Oct;230(4):313-7.

29. Nilsson J, Volk-Jovinge S, Svensson J, Landou C, De Faire U, Hamsten A. Association between high levels of growth factors in plasma and progression of coronary atherosclerosis. J Intern Med. 1992 Nov;232(5):397-404.

30. Chowdhury M, Ghosh J, Slevin M, Smyth J V, Alexander MY, Serracino-Inglott F. A comparative study of carotid atherosclerotic plaque microvessel density and angiogenic growth factor expression in symptomatic versus asymptomatic patients. Eur J Vasc Endovasc Surg. Elsevier Ltd; 2010 Apr;39(4):388-95.

31. Myllärniemi M, Calderon L, Lemström K, Buchdunger E, Häyry P. Inhibition of platelet-derived growth factor receptor tyrosine kinase inhibits vascular smooth muscle cell migration and proliferation. FASEB J. 1997 Nov;11(13):1119-26.

32. Sano H, Sudo T, Yokode M, Murayama T, Kataoka H, Takakura N, et al. Functional blockade of plateletderived growth factor receptor-beta but not of receptor-alpha prevents vascular smooth muscle cell accumulation in fibrous cap lesions in apolipoprotein E-deficient mice. Circulation. 2001 Jun 19;103(24):2955-60. 
33. Gersuk GM, Westermark B, Mohabeer AJ, Challita PM, Pattamakom S, Pattengale PK. Inhibition of human natural killer cell activity by platelet-derived growth factor (PDGF). III. Membrane binding studies and differential biological effect of recombinant PDGF isoforms. Scand J Immunol. 1991 May;33(5):521-32.

34. Zohlnhöfer D, Hausleiter J, Kastrati A, Mehilli J, Goos C, Schühlen H, et al. A randomized, double-blind, placebo-controlled trial on restenosis prevention by the receptor tyrosine kinase inhibitor imatinib. J Am Coll Cardiol. 2005 Dec 6;46(11):1999-2003. 


\section{Chapter 5}

\section{Angiopoietin-2 blocking antibodies reduce early atherosclerotic plaque development in mice}

Theelen TL*, Lappalainen JP*, Sluimer JC*, Gurzeler E, Cleutjens JP, Gijbels MJ, Biessen EA, Daemen MJ, Alitalo K, Ylä-Herttuala S

*Authors contributed equally Modified from Atherosclerosis. 2015 Aug;241(2):297-304. 


\section{ABSTRACT}

\section{Objective}

Angiopoietin-2 (Ang-2) blocking agents are currently undergoing clinical trials for use in cancer treatment. Ang-2 has also been associated with rupture-prone atherosclerotic plaques in humans, suggesting a role for Ang-2 in plaque stability. Despite the availability of Ang-2 blocking agents, their clinical use is still lacking. Our aim was to establish if Ang-2 has a role in atheroma development and in the transition of subclinical to clinically relevant atherosclerosis. We investigated the effect of antibody-mediated Ang-2 blockage on atherogenesis in a mouse model of atherosclerosis.

\section{Approach and results}

Hypercholesterolemic (low-density lipoprotein receptor-/- apolipoprotein $\mathrm{B}^{100 / 100}$ ) mice were subjected to high-cholesterol diet for eight weeks, one group with and one group without Ang-2 blocking antibody treatment during weeks 4-8. To enhance plaque development, a peri-adventitial collar was placed around the carotid arteries at the start of antibody treatment. Aortic roots, carotid arteries and brachiocephalic arteries were analyzed to evaluate the effect of Ang-2 blockage on atherosclerotic plaque size and stable plaque characteristics.

Anti-Ang- 2 treatment reduced the size of fatty streaks in the brachiocephalic artery $(-72 \%$, $\mathrm{p}<0.05)$. In addition, antibody-mediated Ang-2 blockage reduced plasma triglycerides ($27 \%, p<0.05)$. In contrast, Ang- 2 blockage did not have any effect on the size or composition (collagen content, macrophage percentage, adventitial microvessel density) of pre-existing plaques in the aortic root or collar-induced plaques in the carotid artery.

\section{Conclusions}

Ang-2 blockage was beneficial as it decreased fatty streak formation and plasma triglyceride levels, but had no adverse effect on pre-existing atherosclerosis in hypercholesterolemic mice. 


\section{INTRODUCTION}

Increased plasma levels of the vascular growth factor angiopoietin-2 (Ang-2) occur in several diseases, including cancer (1), systemic infections (2), and in cardiovascular diseases such as acute myocardial infarction (3). Ang-2 is also increased in highly vascularized, ruptureprone human atherosclerotic plaques $(4,5)$. Despite the detrimental role of Ang-2 in atherosclerosis suggested by these results, Ang-2 overexpression decreased plaque size in a mouse model of atherosclerosis (6). However, we considered that systemic Ang-2 overexpression fails to mimic the function of endogenous Ang-2 in atherogenesis.

Despite increased Ang-2 levels in clinically relevant atherosclerosis, and the availability of Ang-2 blocking agents, there have been no attempts to study Ang-2 blockage in cardiovascular diseases. Therefore, we considered it important to study the role of Ang-2 blocking as a therapeutic strategy in atherosclerosis. Because the blocking of vascular endothelial growth factor in cancer therapy increased the risk of unpredicted cardiovascular side effects, such as arterial thrombosis, heart failure or cardiomyopathy (7-10), and because anti-Ang-2 therapy is currently used for cancer treatment as well, we aimed to evaluate the potential cardiovascular risk of anti-Ang-2 treatment in an experimental setting of pre-existing atherosclerosis. In order to investigate the role of endogenous Ang2, a blocking strategy was adopted. Currently, a wide variety of Ang-2 blocking agents are being tested in clinical trials already in Phase III against different types of cancer (reviewed in (11)). We used an antibody that inhibits tumor growth in mice by blocking Ang-2 binding to its receptor Tie-2 (12).

The therapeutic potential of Ang-2 blockage is based on the adverse effects of Ang-2 on processes leading to plaque instability: microvessel growth (angiogenesis), decreased pericyte coating of microvasculature and increased vascular permeability $(2,13,14)$, that promotes leukocyte recruitment $(13,15,16)$. In addition, Tie- 2 expressing macrophages respond to Ang-2 by secreting tumor necrosis factor $\alpha$ (TNF $\alpha$ ) (17), which can contribute to the inflammation in the vessel wall.

Microvessel growth and permeability, and inflammation are also involved in the transition of a clinically silent, stable atherosclerotic plaque into a vulnerable plaque at risk of fibrous cap rupture and ensuing clinical events (18). The abundance and compromised structural integrity of plaque microvessels are detrimental to plaque stability, increasing the likelihood of plaque rupture (18-20). Structurally abnormal microvessels involve the risk of plaque hemorrhage and lead to increased lipoprotein deposition and influx of leukocytes, triggering a pro-inflammatory cycle that results in plaque weakening.

In this study, we investigated the effects of the Ang-2 blocking antibody on fatty streak formation and atherosclerotic plaque progression in aortic root, brachiocephalic arteries and in collar-induced carotid atherosclerosis in $\mathrm{LDLr}^{-/-} \mathrm{ApoB}^{100 / 100}$ mice. 


\section{MATERIALS AND METHODS}

\section{Experimental Animals}

Ten to twelve week old male $\mathrm{LDLr}^{-/} \mathrm{ApoB}^{100 / 100}$ mice $(n=26)$ were placed on a high cholesterol diet ( $42 \%$ of calories from fat and $0.15 \%$ from cholesterol, no sodium cholate; TD 88173 Harlan Teklad, Boxmeer, NL) for 8 weeks. After 3 weeks of diet, mice underwent collar placement surgery as previously described (21). In brief, the mice were anaesthetized with isoflurane (induction: $4.5 \%$ isoflurane, $450 \mathrm{ml}$ air, maintenance: $2.0 \%$ isoflurane, 200 $\mathrm{ml}$ air; Baxter International, Helsinki, Finland) and injected with $0.1 \mathrm{mg}$ Rimadyl s.c. (Pfizer, Helsinki, FI). Anesthesia was controlled regularly by visual inspection and toe pinch reflex. Carotid arteries were accessed via a sagittal anterior neck incision and dissected from the surrounding tissue without damaging the vagus nerve or the carotid arteries. A $2 \mathrm{~mm}$ silastic collar was placed bilaterally, right under the bifurcation and fixed with three circumferential surgical silk knots.

One day after collar placement surgery, the mice were distributed randomly and injected intraperitoneally with either a monoclonal Ang-2 blocking antibody (12) $(n=14)$ or a control immunoglobulin ( $\mathrm{n}=12 ; \operatorname{lgG}$ ) at a dose of $10 \mathrm{mg} / \mathrm{kg}$, as previously described (22). The injections were repeated three times weekly over a total time of five weeks.

Food and water were provided ad libitum during the entire study. All animal experiments were approved by National Experimental Animal Board of Finland and carried out in accordance with guidelines of the Finnish Act on Animal Experimentation.

\section{Plasma Ang-2 levels}

Plasma Ang-2 concentrations were measured using enzyme linked immunosorbent assay for murine Ang-2 (R\&D systems, Abingdon, UK).

\section{Echocardiography and carotid artery ultrasound}

Echocardiographic measurements were performed before the collar operations and at 5 weeks after the operation/antibody treatment using the Vevo ${ }^{\circledR} 2100$ Ultrasound System (VisualSonics ${ }^{\circledR}$, Amsterdam, NL). The animals were anesthetized with isoflurane. The Ejection fraction (EF), fractional shortening (FS), left ventricle (LV) mass, LV diastolic and systolic volume were analyzed from parasternal short axis M-mode measurements. EF was calculated by Vevo2100 software using the Teicholz formula (23). Carotid strain was measured as percent change in the arterial diameter: (SD - DD)/DD, where SD was the systolic and DD the diastolic CCA diameter. Peak wall shear stress at $1 \mathrm{~mm}$ proximal to the collar was calculated by the Poiseuille equation: $\tau($ dyn/cm2 $)=4 \times V \times \eta / I D$, where $V$ is the peak systolic flow velocity (cm/s), $\eta$ is the blood viscosity (taken as 0.035 poise), and ID is the maximal lumen diameter of the targeted carotid artery $(\mathrm{cm})(24,25)$.

\section{Total serum cholesterol and triglycerides}

Plasma was separated by centrifugation and stored at $-80^{\circ} \mathrm{C}$ until further use. Cholesterol was determined using standard enzymatic assays (CHOD-PAP method - Cholesterol FS Ecoline product no. 113009990 314, DiaSys, Holzheim, GE), as were triglyceride levels (GPO method - Triglycerides FS Ecoline REF 157609990314 both DiaSys). 


\section{Atherosclerotic plaque quantification and immunohistochemistry}

Atherosclerotic plaque development in the murine arterial tree starts in the aortic root, extending to the aortic arch and brachiocephalic trunk and, after an extended time period, develop into the carotid bifurcation (26). In this study, the mice were fed a high cholesterol diet for 8 weeks and subjected to antibody treatment during the last 5 weeks. The plaques in the brachiocephalic artery consisted mainly of foamy macrophages without an overlying cap or extracellular cholesterol crystals, thus termed fatty streaks. The plaques in the aortic root and carotid arteries were classified as advanced plaques composed of immune cells, smooth muscle cells and a necrotic core, all covered by a fibrous cap. The antibody effect on fatty streak formation could thus be studied in the brachiocephalic artery, while the effect on progression of pre-existing, advanced plaques was studied in the aortic root and carotid arteries with a collar placement, respectively.

Mice were euthanized by $\mathrm{CO}_{2}$ asphyxiation and blood was collected via the right ventricle for ELISA, total cholesterol- and triglyceride analysis. Remaining blood was cleared by perfusion with $20 \mathrm{ml}$ PBS via the left ventricle. Brachiocephalic arteries, aortic root and right common carotid arteries were excised and immersion fixed in $1 \%$ paraformaldehyde overnight.

Tissue samples were paraffin embedded, serially sectioned $(4 \mu \mathrm{m})$ and stained with hematoxylin and eosin ( $\mathrm{HE}$, Sigma, Zwijndrecht, $\mathrm{NL}$ ) for quantification of the plaque areas using computerized morphometry (Leica QWin V3, Cambridge, UK). Total plaque area and necrotic core content were obtained by averaging measurements of five representative sections of the brachiocephalic artery, aortic root and right common carotid artery. The necrotic core was defined by cholesterol clefts, lipid droplets and acellular regions. Collagen content was determined from representative sections stained with Sirius Red (Sirius Red ${ }^{+}$ area/plaque area; Sigma). Plaque macrophages and the intra-plaque and adventitial microvessels were quantified using immunohistochemistry for Mac-3 (Mac- $3^{+}$area/plaque area; BD Pharmingen, Breda, NL) and CD31 (BD Pharmingen, Breda, NL) respectively.

\section{Statistical analysis}

All data are presented as mean \pm SEM. Following Shapiro-Wilk test for normal distribution, the groups were compared with student's t-test or Mann-Whitney rank-sum test. (GraphPad Prism4, La Jolla, CA, USA). A p-value of $p<0.05$ was considered significant. 


\section{RESULTS}

\section{Ang-2 blocking decreases plasma triglycerides but not plasma cholesterol levels}

The Ang2 antibody used in the study blocks the Ang-2 fibrinogen binding domain that is responsible for receptor binding (12), and this results in increased Ang-2 levels in the circulation (27). The antibody injections resulted in an almost 30 -fold increased plasma Ang2 concentration after five weeks of treatment (Figure 1A). An increase in plasma Ang- 2 has also been described in humans treated with the anti-Ang-2 antibody (27). During five weeks of administration, the antibody treatment did not affect the general health status, including body weight (Figure 1B), cardiac function (Figures 1E-J) or shear stress in carotid artery after collar implantation (Figures $1 \mathrm{~K}$ and $\mathrm{L}$ ). However, the treatment led to a $27 \%$ decrease in plasma triglycerides (Figure 1D) without any effects on plasma cholesterol (Figure 1C).
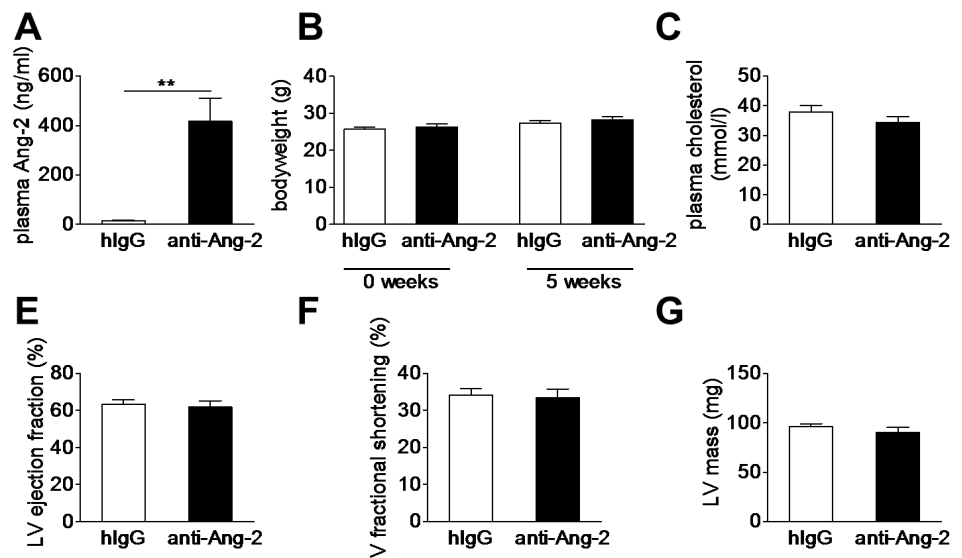

$\mathbf{F}$
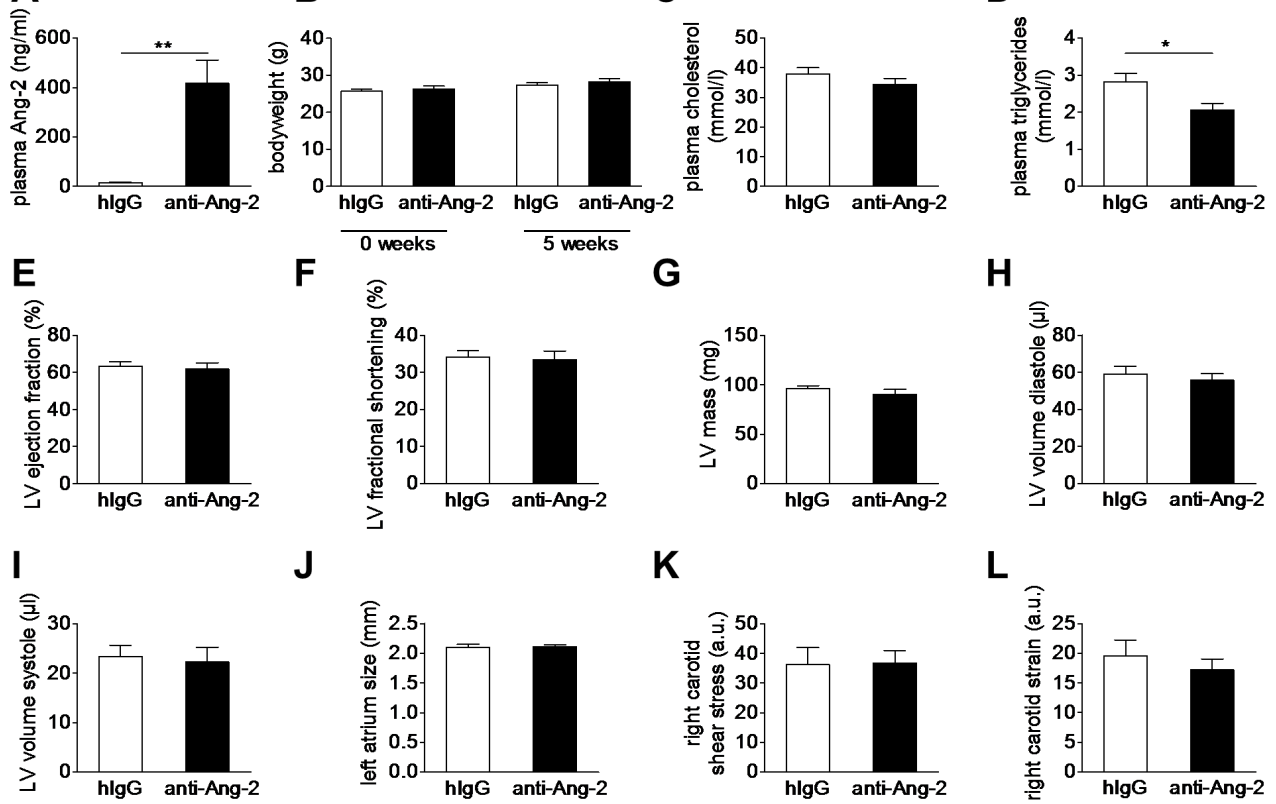

H

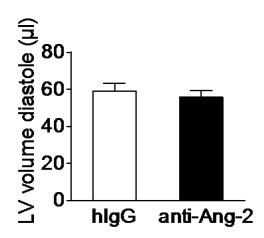

$\mathbf{L}$

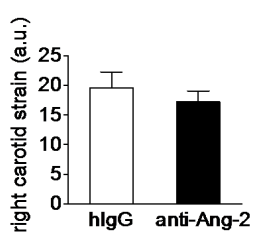

Figure 1. Anti Ang-2 antibody treatment increases plasma Ang-2 levels and decreases plasma triglycerides Plasma Ang-2 levels in anti-Ang and IgG treated mice (A). During the five weeks of administration, the antibody treatment did not affect the general health status, body weight (B), cardiac function (E-J) or shear stress in carotid artery after collar implantation $(\mathbf{K}+\mathbf{L})$. However, the treatment led to a decrease in plasma triglycerides (D) without effects on plasma cholesterol (C). Mean \pm SEM, ${ }^{*} p<0.05 ; * * p<0.01$ 


\section{Ang-2 blocking decreases fatty streak formation}

In order to investigate if Ang-2 blockage affects fatty streak formation, the brachiocephalic artery (28) was analyzed after eight weeks of high-cholesterol diet. Interestingly, fatty streak formation in the brachiocephalic artery was significantly less in mice treated with the anti-Ang-2 antibody than control IgG (Figures 2A-C). While anti-Ang-2 therapy reduced the percentage of stenosis, the lumen and media size remained unchanged (Figures 2D-F). Furthermore, Ang-2 blockage did not alter the fatty streak composition: the collagen content and the percentage of Mac-3 positive macrophages in the plaques (Figures 2G-I and $\mathrm{J}-\mathrm{L}$, respectively) remained unaltered. No microvessels were found in the fatty streaks and adventitial microvessel density was not altered by the antibody treatment (Figures $2 \mathrm{M}-\mathrm{O}$ and data not shown). 
A
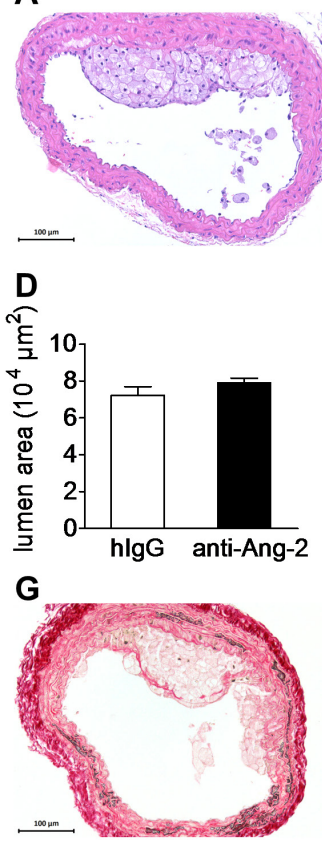

J

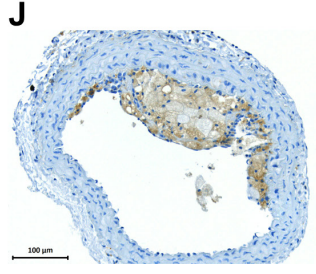

B

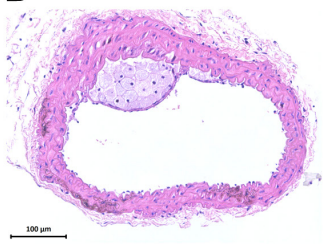

E
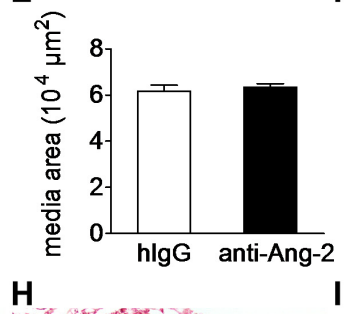

$F$

I
$\mathrm{C}^{\mathrm{T}}$
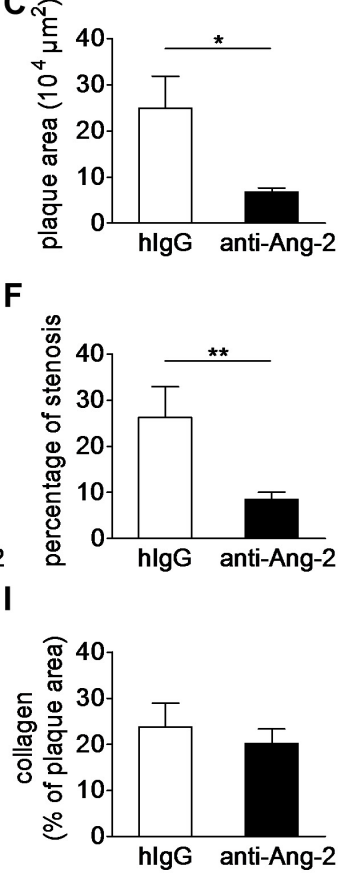

L

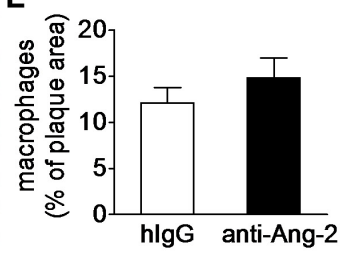

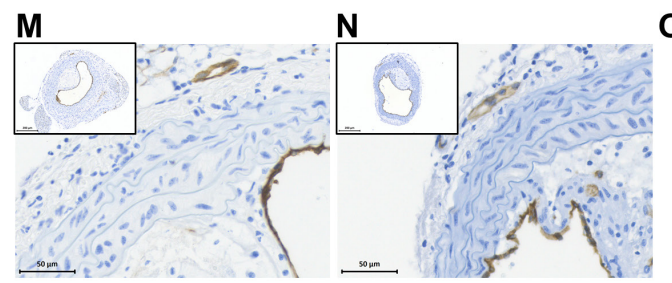

0

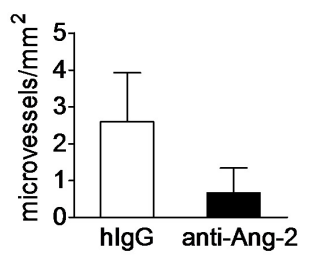

Figure 2. Antibody mediated Ang-2 blockage delays fatty streak formation in the brachiocephalic artery

Representative images of HE-stained brachiocephalic arteries (A+B) show a decrease in fatty streak size in animals treated with anti Ang-2 antibody (C). There is no difference in lumen area or medial thickness (D+E) but a reduction in stenosis after anti-Ang-2 treatment (F). Plaque collagen content (percentage of Sirius Red positive area/total plaque area, G-I), percentage of macrophage area (Mac3+ area/total plaque area, $\mathrm{J}-\mathrm{L}$ ) and adventitial microvessel density (microvessels $/ \mathrm{mm}^{2}$ ) (MVD) (M-O).. Representative images: left panels IgG, right panels anti-Ang-2 antibody. Mean $\pm S E M, * p<0.05$ 


\section{Existing and advanced plaques are not affected by antibody-mediated Ang-2 blockage}

Mice were fed a high-cholesterol diet for three weeks followed by anti-Ang-2 antibody treatment while the diet was continued. This allowed us to analyze the pre-existing plaques in the aortic root in addition to fatty streaks in the brachiocephalic artery. Plaque sizes in aortic roots of mice treated with anti-Ang-2 did not differ from control IgG injected mice after eight weeks of high- cholesterol diet and five weeks of antibody administration (Figures $3 \mathrm{~A}-\mathrm{C}$ ). In addition, the treatment did not alter the amount of necrotic core (Figures $3 \mathrm{~A}, \mathrm{~B}$ and $\mathrm{D}$ ), collagen content (Figures $3 \mathrm{E}-\mathrm{G}$ ) or the macrophages in the plaques (Figures $3 \mathrm{H}$ $J)$. No changes in intra-plaque vessels or adventitial microvessel density were observed (Figures 3K-M and data not shown). Hence, Ang-2 blockage did not interfere with the progression or stability of pre-existing plaques.

In addition to the pre-existing plaques in the aortic root, we were interested in the effects of the antibody on collar-induced fast-growing advanced plaques. The plaque size and necrotic core content in these rapidly progressing collar-induced advanced plaques did not differ between the anti-Ang-2 and control IgG-treated mice (Figures 4A-D). The effect of anti-Ang-2 on fatty streak formation and atherosclerotic plaque development has been summarized in Table 1. 

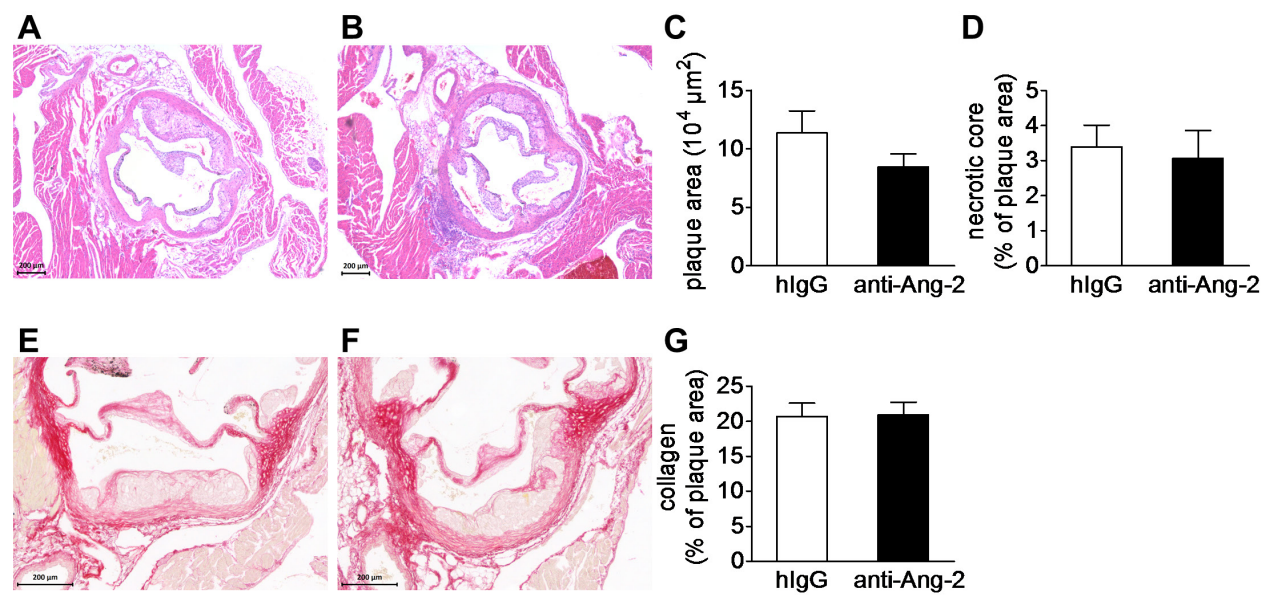

G
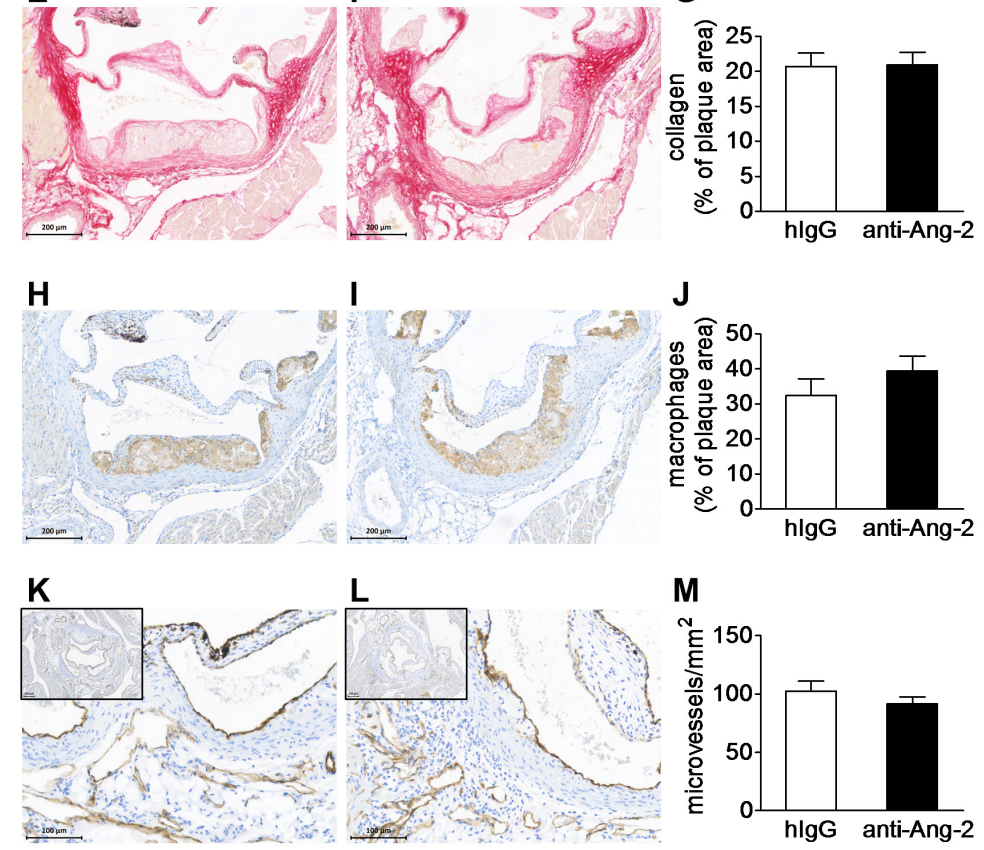

Figure 3. Anti Ang-2 antibody treatment does not alter pre-existing atherosclerosis in the aortic root

Representative images of $\mathrm{HE}$-stained plaques in the aortic root $(\mathbf{A}+\mathbf{B})$ show no difference in plaque size (C) or necrotic core (D) in pre-existing plaques of the aortic root after antibody treatment. Also, collagen (percentage of Sirius Red positive area/total plaque, E-G), macrophage content (Mac3+ area/total plaque area, H-J) and adventitial microvessel density (microvessels $/ \mathrm{mm}^{2}, \mathbf{K}-\mathbf{M}$ ) were not altered by the anti-Ang-2 antibody treatment. Representative pictures: left panels control IgG, right panels anti-Ang-2. Mean $\pm S E M$ 
A

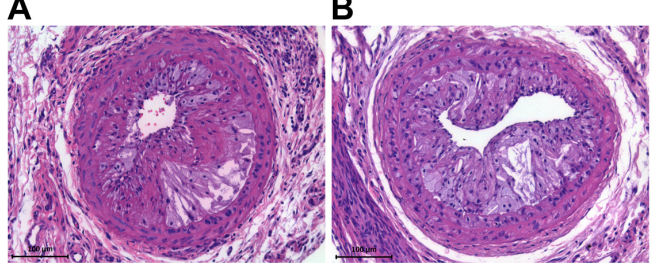

C

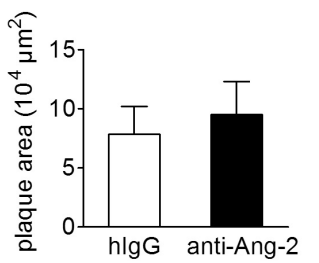

D

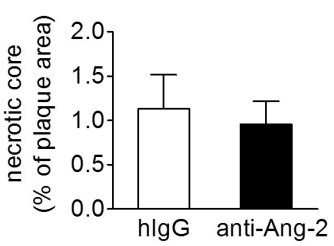

Figure 4. Advanced lesions in the carotid arteries are not affected by Ang-2 blockage

Representative images of HE-stained collar induced advanced plaques $(A+B)$ in the right common carotid artery. Plaque size (C) necrotic core (D). Representative images and quantification of (E-G) collagen, (H-J) macrophages, (K-M) microvessels: left control IgG, right anti-Ang-2. Mean \pm SEM

\begin{tabular}{|c|c|c|c|c|}
\hline & Parameter & Tissue & Alteration in anti-Ang-2 mice & Statistics \\
\hline \multirow[t]{4}{*}{ Plaque burden } & \multirow[t]{3}{*}{ Plaque size } & Brachiocephalic artery & $\downarrow$ & ${ }^{*} p<0.05$ \\
\hline & & Collar model & $=$ & n.s. \\
\hline & & Aortic root & $=$ & n.s. \\
\hline & Degree of stenosis & Brachiocephalic artery & $\downarrow$ & ** $p<0.01$ \\
\hline \multirow[t]{9}{*}{ Plaque phenotype } & \multirow[t]{3}{*}{ Necrotic core } & Brachiocephalic artery & absent in fatty streak & - \\
\hline & & Collar model & $=$ & n.s. \\
\hline & & Aortic root & $=$ & n.s. \\
\hline & \multirow[t]{2}{*}{ Plaque collagen } & Brachiocephalic artery & $=$ & n.s. \\
\hline & & Aortic root & $=$ & n.s. \\
\hline & \multirow[t]{2}{*}{ Plaque macrophages } & Brachiocephalic artery & $=$ & n.s. \\
\hline & & Aortic root & $=$ & n.s. \\
\hline & \multirow[t]{2}{*}{ Microvessel density } & Brachiocephalic artery & $=$ & n.s. \\
\hline & & Aortic root & $=$ & n.s. \\
\hline \multirow{6}{*}{$\begin{array}{l}\text { Systemic effects } \\
\text { of anti-Ang-2 }\end{array}$} & Angiopoetin-2 & Plasma & $\uparrow$ & ${ }^{* *} p<0.01$ \\
\hline & Cholesterol & Plasma & $=$ & n.s. \\
\hline & Triglycerides & Plasma & $\downarrow$ & ${ }^{*} p<0.05$ \\
\hline & Cardiac function & US of the heart & $=$ & n.s. \\
\hline & Carotid strain+shear stress & US of carotid artery & $=$ & n.s. \\
\hline & Bodyweight & Before sacrifice & $=$ & n.s. \\
\hline
\end{tabular}




\section{DISCUSSION}

Our study is the first one to investigate Ang-2 depletion in experimental atherosclerosis and the results demonstrate that antibody-mediated Ang-2 depletion reduces fatty streak formation in the brachiocephalic arteries. In addition, anti-Ang-2 treatment reduces plasma triglycerides while plasma cholesterol levels remain unchanged. The anti-Ang-2 antibody did not interfere with pre-existing plaques of the aortic root or with collar-induced plaques in the carotid artery. Moreover, Ang-2 depletion had no effect on the stability of the plaque phenotype as microvessel density, macrophage and collagen content and the necrotic cores remained unchanged (Table 1 ).

\section{Ang-2 loss-of-function model contradictory to gain-of-function model in atherosclerosis}

To date, inhibition or knock-out of endogenous Ang-2 in human or murine atherosclerosis have not been reported. However, Ang-2 overexpression was shown to decrease plaque formation in the aortic root of ApoE knock-out mice via inhibition of LDL oxidation (6). However, the impact on plaque stability was not studied in great detail. The authors only observed an absolute decrease in plaque macrophages after Ang-2 overexpression, albeit that data were not corrected for the reduced overall plaque size (6). Therefore, the reduction in macrophage area probably only reflects the plaque stage. Concordant with this notion, in our study, we did not observe any difference in relative macrophage content (macrophage area normalized to the plaque area) after Ang-2 blockage. Enhancing Ang-2 may seem an attractive therapy at first sight. However, transgenic Ang-2 overexpression does not mimic the endogenous role of Ang-2 in atherogenesis, which is crucial for the translation to the human situation.

\section{No role for Ang-2 blockage in plaque stabilization}

Considering the stimulatory role of Ang-2 in various processes associated with vulnerable plaques (18), such as increased inflammation (13), plaque vascularity (5), microvascular leakage (15) and matrix metalloprotease expression (5), we expected profound effects of Ang-2 antagonism on the stable plaque phenotype. However, Ang-2 deletion was only able to prevent fatty streak formation; it did not prevent plaque progression or changes in plaque stability.

Changes in fatty streak formation could not be attributed to effects on plaque inflammation or angiogenesis. Rather, Ang-2 blockage may retard fatty streak formation by a reduction in plasma triglycerides, prevention of endothelial dysfunction or reduction of monocyte chemotaxis. We observed lower plasma triglycerides after Ang-2 antibody treatment, which might explain the decrease in fatty streak formation. Until recently, hypertriglyceridemia was regarded as a risk factor of cardiovascular disease based on epidemiological association only, with much controversy on possible causality. However, recent Mendelian randomization studies increasingly support the causative role for increased plasma triglycerides in cardiovascular disease and atherosclerosis $(29,30)$. Cellular effects are mediated by remnant lipoproteins, rich in triglycerides, rather than chylomicrons (reviewed in (31)). Mechanistically, direct uptake of these remnant lipoproteins by macrophages stimulates cholesterol ester accumulation and macrophage foam cell formation, an important step in fatty streak formation (32). Neutral and oxidized free fatty acids generated by lipolysis of these triglyceride-rich lipoproteins can also stimulate 112 
endothelial dysfunction, subsequently enhancing vascular inflammation and lipoprotein retention (33), and hence fatty streak formation (31). Thus, lower triglycerides may prevent endothelial dysfunction and foam cell formation, explaining the reduction in fatty streak formation. Although high triglyceride levels have also been associated with clinically relevant, advanced stages of atherosclerosis (34), reduced levels were without effect on pre-existing and advanced atherosclerosis in our model.

Ang-2 blockage could decrease fatty streak formation also via processes that are regulated by the angiopoietin receptor Tie2, which is highly expressed in endothelial cells and monocytes, both involved in the early development of atherosclerosis. Ang- 2 expressed by endothelial cells has been shown to stimulate monocyte chemotaxis (35), hence Ang-2 blockage may reduce monocyte recruitment to developing fatty streaks. However, as we did not observe alterations in macrophage content of fatty streaks, this seems unlikely. More likely is improvement of of endothelial cell dysfunction and the subsequent permeability for lipoproteins by anti-Ang-2. This is thought to be the initial step at the onset of atherosclerosis. Ang-2 is known to induce endothelial destabilization and vascular permeability (36), thus suggesting a beneficial effect of Ang-2 blockage.

\section{Used Ang-2 antibody proven to be effective in other disease models}

The Ang-2 blocking capacity of anti-Ang2 antibodies in vitro (12) and in vivo $(12,22,27,37)$ has been firmly established and was confirmed in our study by using Ang-2 ELISA. It has been reported that treatment of cardiac allografts with anti-Ang2 protects from transplant inflammation and rejection (37). This is in line with atherosclerosis inhibition by overexpression of the Tie2 agonist ligand, Ang1 in cardiac allografts (38). Antibodymediated Ang-2 blockage has been proven to reduce tumor growth (12) and lung metastasis formation (22) in murine cancer models by increasing tumor vessel stability. Moreover, Ang2 is released by endothelial cells (39), thus anti-Ang- 2 antibody has perfect access to the target through the adventitial microvasculature and the main arteries. It is thus unlikely that Ang-2 depletion or impaired tissue availability of the antibody could explain the lack of effect on plaque progression and stable plaque phenotype.

\section{Inhibiting Ang-2 alone might not be sufficient}

Alternatively, inhibiting Ang-2 only might not suffice to affect pre-existing atherosclerosis. Additional factors, such as vascular endothelial growth factor A (VEGF-A) should be targeted as well. For example, one could employ a bifunctional anti-VEGF-A/anti-Ang-2 antibody, which combines the inhibition of both growth factors; this has already provided a stronger anti-tumor effect than targeting the two growth-factors separately (40).

\section{Ang-2 cause or consequence of atherosclerosis}

The lack of effect on plaque growth or the stable plaque phenotype is in contrast to previously reported associations between Ang-2 and cardiovascular disease. Increased Ang2 plasma levels have been correlated with cardiovascular disease progression, but have also been suggested to provide a biomarker for future cardiovascular events such as myocardial infarction or stroke $(3,41-47)$. Furthermore, Ang-2 was detected in advanced vascularized human plaques (48) and it was associated with a high microvessel content and destabilizing MMP-2 expression (5). The outcomes of these studies support our findings that Ang-2 depletion helps to inhibit atherosclerosis development. Nevertheless, not all studies could 
show a correlation of Ang-2 with plaques at risk of rupture $(49,50)$. Perhaps the clinical correlation of Ang-2 expression and atherosclerosis reflects a compensatory expression in response to plaque vulnerability. Overall, Ang- 2 may be correlated with cardiovascular diseases, but the results described in the present work do not support a causal role for it in atherosclerosis progression or plaque vulnerability. Importantly, our study gives no reason to think that Ang-2 blockage could have adverse effects on pre-existing atherosclerosis of treated cancer patients.

\section{Conclusions}

This is the first time that Ang- 2 blockage has been tested in an experimental model of atherosclerosis and found to have significant protective effects on fatty streak formation. Ang-2 blockage reduces plasma triglycerides and decreases early plaque formation, suggesting a beneficial effect of Ang-2 depletion on the early phase of atherosclerosis. In addition, no effects on pre-existing atherosclerosis or cardiac function were found, suggesting that anti-Ang-2 therapy is safe in various clinical settings. 


\section{ACKNOWLEDGEMENTS}

The authors would like to thank Dr. ChingChing Leow and Medlmmune for the anti-Ang2 antibodies, and the staff of the Experimental animal facility at the University of Eastern Finland, Kuopio. Research was supported by Finnish Academy, ERC grant, Sigrid Juselius Foundation, Finnish Foundation for Cardiovascular Research (all to S.Y.); the European Research Council (ERC-2010-AdG-268804, VESSEL network), Leducq Transatlantic Network of Excellence on Lymph Vessels in Obesity and Cardiovascular Disease (11CVD03) (all to K.A.), a VENI fellowship of the Netherlands Organization of Scientific research (to J.C.S. 016.116.017); a PhD-student fellowship from the Cardiovascular Research Institute Maastricht (to T.L.T.). 


\section{REFERENCES}

1. Llovet JM, Peña CEA, Lathia CD, Shan M, Meinhardt G, Bruix J, et al. Plasma biomarkers as predictors of outcome in patients with advanced hepatocellular carcinoma. Clin Cancer Res. 2012 Apr 15;18(8):2290300 .

2. Ziegler T, Horstkotte J, Schwab C, Pfetsch V, Weinmann K, Dietzel S, et al. Angiopoietin 2 mediates microvascular and hemodynamic alterations in sepsis. J Clin Invest. 2013 Jul 1;

3. Iribarren C, Phelps BH, Darbinian JA, McCluskey ER, Quesenberry CP, Hytopoulos E, et al. Circulating angiopoietins-1 and -2, angiopoietin receptor Tie-2 and vascular endothelial growth factor-A as biomarkers of acute myocardial infarction: a prospective nested case-control study. BMC Cardiovasc Disord. 2011;11(1):31.

4. Le Dall J, Ho-Tin-Noé B, Louedec L, Meilhac O, Roncal C, Carmeliet P, et al. Immaturity of microvessels in haemorrhagic plaques is associated with proteolytic degradation of angiogenic factors. Cardiovasc Res. 2010 Jan 1;85(1):184-93.

5. Post S, Peeters W, Busser E, Lamers D, Sluijter JPG, Goumans M-J, et al. Balance between angiopoietin-1 and angiopoietin-2 is in favor of angiopoietin-2 in atherosclerotic plaques with high microvessel density. J Vasc Res. 2008;45(3):244-50.

6. Ahmed A, Fujisawa T, Niu X-L, Ahmad S, Al-Ani B, Chudasama K, et al. Angiopoietin-2 confers Atheroprotection in apoE-/- mice by inhibiting LDL oxidation via nitric oxide. Circ Res. 2009 Jun 19;104(12):1333-6.

7. Bowles EJA, Wellman R, Feigelson HS, Onitilo AA, Freedman AN, Delate T, et al. Risk of heart failure in breast cancer patients after anthracycline and trastuzumab treatment: a retrospective cohort study. J Natl Cancer Inst. 2012 Sep 5;104(17):1293-305.

8. Chen J, Long JB, Hurria A, Owusu C, Steingart RM, Gross CP. Incidence of heart failure or cardiomyopathy after adjuvant trastuzumab therapy for breast cancer. J Am Coll Cardiol. 2012 Dec 18;60(24):2504-12.

9. Choueiri TK, Mayer EL, Je Y, Rosenberg JE, Nguyen PL, Azzi GR, et al. Congestive heart failure risk in patients with breast cancer treated with bevacizumab. J Clin Oncol. 2011 Feb 20;29(6):632-8.

10. Scappaticci FA, Skillings JR, Holden SN, Gerber H-P, Miller K, Kabbinavar F, et al. Arterial thromboembolic events in patients with metastatic carcinoma treated with chemotherapy and bevacizumab. J Natl Cancer Inst. 2007 Aug 15;99(16):1232-9.

11. Saharinen P, Eklund L, Pulkki K, Bono P, Alitalo K. VEGF and angiopoietin signaling in tumor angiogenesis and metastasis. Trends Mol Med. 2011 Jul;17(7):347-62.

12. Leow CC, Coffman K, Inigo I, Breen S, Czapiga M, Soukharev S, et al. MEDI3617, a human anti-angiopoietin 2 monoclonal antibody, inhibits angiogenesis and tumor growth in human tumor xenograft models. Int J Oncol. 2012 May;40(5):1321-30.

13. Fiedler U, Reiss $Y$, Scharpfenecker M, Grunow V, Koidl S, Thurston G, et al. Angiopoietin-2 sensitizes endothelial cells to TNF-alpha and has a crucial role in the induction of inflammation. Nat Med. 2006 Feb;12(2):235-9.

14. Lobov IB, Brooks PC, Lang RA. Angiopoietin-2 displays VEGF-dependent modulation of capillary structure and endothelial cell survival in vivo. Proc Natl Acad Sci U S A. 2002 Aug 20;99(17):11205-10.

15. Benest A V, Kruse K, Savant S, Thomas M, Laib AM, Loos EK, et al. Angiopoietin-2 is critical for cytokineinduced vascular leakage. Parikh SM, editor. PLoS One. 2013 Aug 5;8(8):e70459.

16. Bhandari V, Choo-Wing R, Lee CG, Zhu Z, Nedrelow JH, Chupp GL, et al. Hyperoxia causes angiopoietin 2mediated acute lung injury and necrotic cell death. Nat Med. 2006 Nov;12(11):1286-93. 
17. Murdoch C, Tazzyman S, Webster S, Lewis CE. Expression of Tie-2 by human monocytes and their responses to angiopoietin-2. J Immunol. 2007 Jun 1;178(11):7405-11.

18. Ylä-Herttuala S, Bentzon JF, Daemen M, Falk E, Garcia-Garcia HM, Herrmann J, et al. Stabilization of atherosclerotic plaques: an update. Eur Heart J. 2013 Nov;34(42):3251-8.

19. Moreno PR, Purushothaman KR, Fuster V, Echeverri D, Truszczynska H, Sharma SK, et al. Plaque neovascularization is increased in ruptured atherosclerotic lesions of human aorta: implications for plaque vulnerability. Circulation. 2004 Oct 5;110(14):2032-8.

20. Sluimer JC, Kolodgie FD, Bijnens APJJ, Maxfield K, Pacheco E, Kutys B, et al. Thin-walled microvessels in human coronary atherosclerotic plaques show incomplete endothelial junctions relevance of compromised structural integrity for intraplaque microvascular leakage. J Am Coll Cardiol. 2009 Apr 28;53(17):1517-27.

21. von der Thüsen JH, van Berkel TJ, Biessen EA. Induction of rapid atherogenesis by perivascular carotid collar placement in apolipoprotein E-deficient and low-density lipoprotein receptor-deficient mice. Circulation. 2001 Feb 27;103(8):1164-70.

22. Holopainen T, Saharinen P, D’Amico G, Lampinen A, Eklund L, Sormunen R, et al. Effects of angiopoietin2-blocking antibody on endothelial cell-cell junctions and lung metastasis. J Natl Cancer Inst. $2012 \mathrm{Mar}$ 21;104(6):461-75.

23. Huusko J, Lottonen L, Merentie M, Gurzeler E, Anisimov A, Miyanohara A, et al. AAV9-mediated VEGF-B gene transfer improves systolic function in progressive left ventricular hypertrophy. Mol Ther. 2012 Dec;20(12):2212-21.

24. Feintuch A, Ruengsakulrach P, Lin A, Zhang J, Zhou Y-Q, Bishop J, et al. Hemodynamics in the mouse aortic arch as assessed by MRI, ultrasound, and numerical modeling. Am J Physiol Heart Circ Physiol. 2007 Feb;292(2):H884-92.

25. Castier Y, Brandes RP, Leseche G, Tedgui A, Lehoux S. p47phox-dependent NADPH oxidase regulates flow-induced vascular remodeling. Circ Res. 2005 Sep 16;97(6):533-40.

26. Lutgens E, Daemen M, Kockx M, Doevendans P, Hofker M, Havekes L, et al. Atherosclerosis in APOE*3Leiden transgenic mice: from proliferative to atheromatous stage. Circulation. 1999 Jan 19;99(2):27683.

27. Herbst RS, Hong D, Chap L, Kurzrock R, Jackson E, Silverman JM, et al. Safety, pharmacokinetics, and antitumor activity of AMG 386, a selective angiopoietin inhibitor, in adult patients with advanced solid tumors. J Clin Oncol. 2009 Jul 20;27(21):3557-65.

28. Lutgens E, Gorelik L, Daemen MJ, de Muinck ED, Grewal IS, Koteliansky VE, et al. Requirement for CD154 in the progression of atherosclerosis. Nat Med. 1999 Nov;5(11):1313-6.

29. Johansen CT, Hegele RA. Using Mendelian randomization to determine causative factors in cardiovascular disease. J Intern Med. 2013 Jan;273(1):44-7.

30. Jørgensen AB, Frikke-Schmidt R, West AS, Grande P, Nordestgaard BG, Tybjærg-Hansen A. Genetically elevated non-fasting triglycerides and calculated remnant cholesterol as causal risk factors for myocardial infarction. Eur Heart J. 2013 Jun;34(24):1826-33.

31. Watts GF, Ooi EMM, Chan DC. Demystifying the management of hypertriglyceridaemia. Nat Rev Cardiol. 2013 Nov;10(11):648-61.

32. Goldstein JL, Ho YK, Brown MS, Innerarity TL, Mahley RW. Cholesteryl ester accumulation in macrophages resulting from receptor-mediated uptake and degradation of hypercholesterolemic canine beta-very low density lipoproteins. J Biol Chem. 1980 Mar 10;255(5):1839-48.

33. Wang L, Gill R, Pedersen TL, Higgins LJ, Newman JW, Rutledge JC. Triglyceride-rich lipoprotein lipolysis 
releases neutral and oxidized FFAs that induce endothelial cell inflammation. J Lipid Res. 2009 Feb;50(2):204-13.

34. Triglyceride Coronary Disease Genetics Consortium and Emerging Risk Factors Collaboration, Sarwar N, Sandhu MS, Ricketts SL, Butterworth AS, Di Angelantonio E, et al. Triglyceride-mediated pathways and coronary disease: collaborative analysis of 101 studies. Lancet (London, England). 2010 May 8;375(9726):1634-9.

35. Scholz A, Lang V, Henschler R, Czabanka M, Vajkoczy P, Chavakis E, et al. Angiopoietin-2 promotes myeloid cell infiltration in a $\beta_{2}$-integrin-dependent manner. Blood. 2011 Nov 3;118(18):5050-9.

36. Hakanpaa L, Sipila T, Leppanen V-M, Gautam P, Nurmi H, Jacquemet G, et al. Endothelial destabilization by angiopoietin-2 via integrin $\beta 1$ activation. Nat Commun. 2015 Jan 30;6:5962.

37. Syrjälä SO, Tuuminen R, Nykänen Al, Raissadati A, Dashkevich A, Keränen MAl, et al. Angiopoietin-2 inhibition prevents transplant ischemia-reperfusion injury and chronic rejection in rat cardiac allografts. Am J Transplant. 2014 May;14(5):1096-108.

38. Nykänen Al, Krebs R, Saaristo A, Turunen P, Alitalo K, Ylä-Herttuala S, et al. Angiopoietin-1 protects against the development of cardiac allograft arteriosclerosis. Circulation. 2003 Mar 11;107(9):1308-14.

39. Augustin HG, Koh GY, Thurston G, Alitalo K. Control of vascular morphogenesis and homeostasis through the angiopoietin-Tie system. Nat Rev Mol Cell Biol. 2009 Mar;10(3):165-77.

40. Kienast Y, Klein C, Scheuer W, Raemsch R, Lorenzon E, Bernicke D, et al. Ang-2-VEGF-A CrossMab, a novel bispecific human IgG1 antibody blocking VEGF-A and Ang-2 functions simultaneously, mediates potent antitumor, antiangiogenic, and antimetastatic efficacy. Clin Cancer Res. 2013 Dec 15;19(24):6730-40.

41. Chung NAY, Makin AJ, Lip GYH. Measurement of the soluble angiopoietin receptor tie-2 in patients with coronary artery disease: development and application of an immunoassay. Eur J Clin Invest. 2003 Jul;33(7):529-35.

42. David S, Kümpers $\mathrm{P}$, Hellpap J, Horn R, Leitolf $\mathrm{H}$, Haller $\mathrm{H}$, et al. Angiopoietin 2 and cardiovascular disease in dialysis and kidney transplantation. Am J Kidney Dis. 2009 May;53(5):770-8.

43. David S, Kümpers P, Lukasz A, Kielstein JT, Haller H, Fliser D. Circulating angiopoietin-2 in essential hypertension: relation to atherosclerosis, vascular inflammation, and treatment with olmesartan/pravastatin. J Hypertens. 2009 Aug;27(8):1641-7.

44. Golledge J, Clancy P, Yeap BB, Hankey GJ, Norman PE. Increased serum angiopoietin-2 is associated with abdominal aortic aneurysm prevalence and cardiovascular mortality in older men. Int J Cardiol. 2013 Aug 20;167(4):1159-63.

45. Lee KW, Lip GYH, Blann AD. Plasma angiopoietin-1, angiopoietin-2, angiopoietin receptor tie-2, and vascular endothelial growth factor levels in acute coronary syndromes. Circulation. 2004 Oct 19;110(16):2355-60.

46. Patel J V, Lim HS, Varughese GI, Hughes EA, Lip GYH. Angiopoietin-2 levels as a biomarker of cardiovascular risk in patients with hypertension. Ann Med. 2008 Jan;40(3):215-22.

47. Wang X, Yong H, Mi L, Bai Y, Guo L, Gao W, et al. Changes and significance of serum angiopoietin-2 levels in patients with coronary heart disease. Biomarkers. 2012 Dec;17(8):745-9.

48. Calvi C, Dentelli P, Pagano M, Rosso A, Pegoraro M, Giunti S, et al. Angiopoietin 2 Induces Cell Cycle Arrest in Endothelial Cells: A Possible Mechanism Involved in Advanced Plaque Neovascularization. Arterioscler Thromb Vasc Biol. 2004 Mar 1;24(3):511-8.

49. Pelisek J, Well G, Reeps C, Rudelius M, Kuehnl A, Culmes M, et al. Neovascularization and angiogenic factors in advanced human carotid artery stenosis. Circ J. 2012;76(5):1274-82. 
50. Chen F, Eriksson P, Kimura T, Herzfeld I, Valen G. Apoptosis and angiogenesis are induced in the unstable coronary atherosclerotic plaque. Coron Artery Dis. 2005 May;16(3):191-7. 
Chapter 5 


\section{Chapter 6}

\section{Histamine and VEGF mediate pro-angiogenic gene expression and endothelial cell junction formation through common cellular regulators}

Laakkonen JP*, Lappalainen JP*, Theelen TL, Toivanen PI, Nieminen T, Kaikkonen MU, Sluimer JC, Ylä-Herttuala S

*Authors contributed equally

Submitted 


\section{ABSTRACT}

\section{Objective}

Histamine and vascular endothelial growth factor (VEGF) are involved in several vascular pathologies. Both factors have been suggested to induce neovessel formation and hyperpermeability. Transient phosphorylation events induced by histamine and VEGF are wellknown but gene regulation leading to angiogenesis remains obscure.

\section{Approach and results}

We show here by RNA-sequencing that histamine and VEGF induce similar transcriptional alterations of genes involved in activation of ECs, cell proliferation and adhesion. Altogether, 76 commonly regulated genes were found in human primary endothelial cells (ECS), representing $\sim 53 \%$ of all VEGF-regulated transcripts and $\sim 26 \%$ of all histamineregulated transcripts. Downstream signaling via VEGF or histamine receptors regulated genes involved in calcium signaling, tight junction (TJ) formation, actin re-organization and cell-ECM adhesion. Signaling via VEGFR2 regulated VEGF-specific transcription factors SNAI2 and MYCN, whereas NR4A1 and RCAN1 expression were commonly regulated by both factors independently on VEGFR2. SNAI2 was further shown to be required for EC proliferation and tube formation, and to mediate expression of the major TJ protein claudin5 together with MYCN and NR4A1. Systemic delivery of VEGF and histamine to C57/BI6 mice corroborated in vivo regulation of these factors. Besides commonly expressed transcription factors affecting EC-EC adhesion, both VEGF and histamine induced cell signaling via PI3KAkt pathway and upregulated HGF expression, known to induce angiogenesis.

\section{Conclusion}

This study demonstrates that in addition to transient phosphorylation cascades, major changes in gene expression profile of ECs are induced by VEGF and histamine leading to proangiogenic cellular processes. Our results increase knowledge of angiogenesis and provide novel treatment targets for vascular pathologies. 


\section{INTRODUCTION}

Vascular endothelial growth factors (VEGFs) and inflammatory mediators histamine, bradykinin and serotonin are well known edematogenic factors. Increased levels of histamine have been reported in response to trauma, allergy, burns and infectious diseases (1), whereas increased VEGF levels have been linked to cancer, vascular anomalies and asthma (2-4). Both histamine and VEGF cause vasodilatation, vascular hyper-permeability and local attraction of inflammatory cells. They have been suggested to be released from degranulated mast cells that are often found near inflammation and angiogenic sites (5). Besides their role in hyper-permeability, VEGF and histamine have been suggested to induce endothelial cell proliferation and angiogenesis $(6,7)$. Histamine induced angiogenesis has been observed in primary endothelial cells (6), rabbit cornea (8) and after subcutaneous implantation of histamine pellets (6). Additionally, defective angiogenesis has been shown in histidine decarboxylase knockout mouse that catalyzes histamine synthesis (9) and after treatment with histamine receptor antagonist (7). Histidine decarboxylase and histamine were also recently found to induce differentiation of hematopoietic bone marrow cells (10). Histamine has four receptors (HR1-4) that belong to G-protein-coupled receptor (GPCR) family, from which HR1 and HR2 are expressed in ECs. VEGFs instead bind to VEGF receptors 1-3 (VEGFR), while neuropilins 1-2 (NRP) and heparan sulfate proteoglycans (HSPGs) act as co-receptors. Several VEGF family members have been identified: VEGF-A, -B, -C -D and placenta growth factor in humans, VEGF-E in a virus and VEGF-F derived from snake venom. VEGFs can also exist in several isoforms that vary according to their HSPG binding affinity (2). VEGFR2 has been suggested to be the main receptor responsible for VEGF-induced angiogenesis. In contrast, for histamine two theories exist i) histamine induces VEGF expression and thereby angiogenesis (9) and ii) histamine induces expression of NR4A1 transcription factor that induces angiogenesis (6). In spite of previous work on histamine and VEGF signaling, and the use of various VEGFs in pro-angiogenic therapy, gene regulation by which these factors induce angiogenesis after initial receptor binding and phosphorylation cascades is largely unknown.

In this study, novel methods including RNA-sequencing (RNA-seq) and multi-photon microscopy were used to define the changes induced by various VEGF family proteins and histamine in primary human ECs and in intact vasculature of C57/Bl6 mice. Novel common molecular players involved in angiogenic processes affecting e.g. EC activation, adhesion and proliferation by histamine and VEGF were found by RNA-seq and validated in functional assays. No increase in expression of VEGFs or VEGFRs by histamine was detected. Downstream signaling cascades via VEGF and histamine receptors were both shown to regulate genes involved in calcium signaling, tight junction (TJ) formation, actin reorganization and cell-extracellular matrix adhesion. Common downstream mediators such as hepatocyte growth factor (HGF) and transcription factor RCAN1 (regulator of calcineurin1) were found to regulate both VEGF and histamine mediated responses in ECs. Downregulation of the major tight junction gene claudin-5 and EC proliferation was regulated by PI3K signaling and transcription factors SNAI2 (Snail family zinc finger 2), MYCN (N-myc proto-oncogene protein) and NR4A1 (nuclear receptor subfamily 4 group A member 1). Regulation of NR4A1 and MYCN was also detected after systemic delivery of VEGF or histamine in C57/BI6 mice. Our data show that both histamine and VEGF induce expression of multiple genes in ECs, including growth factors and transcription factors that regulate cell 
Chapter 6

proliferation. Understanding the molecular mechanisms of angiogenic processes in ECs and their underlying gene regulation is crucial for the development of novel ways to reduce edema in pathological conditions and to develop pro- and anti-angiogenic therapies. 


\section{METHODS}

\section{Materials}

Recombinant human VEGF-A $A_{121}$, VEGF-A $A_{165}$, VEGF-D ${ }^{\triangle N \Delta C}$ and VEGF-F were produced and purified as previously described $(11,12)$. Human umbilical vein endothelial cells (HUVECs) were isolated from umbilical cords as previously described (13). Human aortic endothelial cells (HAECs) were purchased from Gibco (Life Technologies). Both cell lines were cultured in Endothelial Cell Growth medium (EGM; Thermo Scientific) on fibronectin-gelatin coated surfaces $(10 \mu \mathrm{g} / \mathrm{ml}, 0.05 \%$; Sigma-Aldrich). Collection of umbilical cords for cell isolation was approved by Ethics Committee of the Kuopio University Hospital (Kuopio, Finland). Silencer Select siRNAs against NR4A1, MET, MYCN, SNAI2, VEGFR2 and two control siRNAs were purchased from Life Technologies.

\section{Experimental animals}

Animal experiments were approved by National Experimental Animal Board of Finland and carried out in accordance with guidelines of the Finnish Act on Animal Experimentation. Recombinant human VEGFs were produced and purified as previously described (11). Histamine was purchased from Sigma-Aldrich (St. Louis, MO). Green fluorescent microspheres (200nm) were obtained from Thermo Fisher Scientific (Fremont, CA). All experiments were performed with 8-12 weeks old male C57/BI6 mice (Harlan Laboratories, Indianapolis, IN). For ex vivo permeability experiments, mice received an intravenous injection of fluorescent microspheres followed by injection with $0.25 \mu \mathrm{g} / \mathrm{g}$ of human recombinant VEGFs or $12.5 \mu \mathrm{g} / \mathrm{g}(4 \mathrm{~h})$ and $25 \mu \mathrm{g} / \mathrm{g}$ (10 min) of histamine under isoflurane anesthesia. Saline was used as control. Mice were sacrificed $10 \mathrm{~min}, 30 \mathrm{~min}$ or $4 \mathrm{~h}$ after injections and perfused with PBS ( $n=4-5$ mice/group). Tissues were harvested and snap frozen in liquid nitrogen for RT-qPCR and western blot. For imaging purposes, tissues were fixed in 4\% PFA in PBS for $2 \mathrm{~h}$, sliced to $1 \mathrm{~mm}$ whole immune mount sections and incubated overnight with rat anti-mouse CD31 (BD Biosciences, Franklin Lakes, NJ), followed by immunostaining with goat anti-rat Alexa594 antibody (Life Technologies, Carlsbad, CA).

\section{Imaging and image analysis}

Imaging was performed by Nikon A1R multi-photon microscope and LSM700 Zeiss confocal microscope. In multi-photon experiments, $25 x / 1.05$ water objective and Chameleon Vision II laser (Coherent, Santa Clara, CA) with an excitation wavelength of $800 \mathrm{~nm}$ was used. The signal was detected at 525-550nm for green fluorescent microspheres and 575-600 nm for CD31 (512x512 frame size). With confocal microscope, 405/488/555nm diode lasers were used together with appropriate emission filters (63x/1.4 PlanApo objective, 512x512 frame size). Image processing and analysis was performed from 3D-images by ImageJ (14) using particle analysis function ( $n=5$ images/animal/tissue).

\section{RNA-Seq Libraries}

Confluent cultures of HUVECs were washed with PBS, followed by starvation of cells for 16 hrs with EGM medium supplemented with $0.5 \% \mathrm{FBS}$. The cells were stimulated with VEGF$\mathrm{A}_{165}(100 \mathrm{ng} / \mathrm{ml})$ or histamine $(20 \mu \mathrm{M})$ for $7 \mathrm{hrs}$. RNA was extracted from HUVEC cells with RNeasy Mini Kit (Qiagen). After enrichment by Poly(A)-RNA with MicroPoly(A) Purist Kit, RNA was fragmented using RNA Fragmentation Reagents (Life Technologies) and purified in 
P-30 column (Bio-Rad, Hercules, CA). Fragmented RNA was further dephosphorylated with PNK (New England Biolabs, Ipswich, MA), heat-inactivated and purified by RNA Clean \& Concentrator ${ }^{\mathrm{TM}}-5$ kit (Zymo Research Corporation). Poly(A)-tailing and CDNA synthesis was performed as described previously (15). For reverse transcription an oligo allowing custom barcoding during final amplification was used: /5Phos/GATCGTCGGACTGTAGAACTCTGAAC/iSp18/TCAGACGTGTGCTCTTCCGATCTTTTTTTT TTTTTTTTTTTTVN (IDT, Coralville, IA). Exonuclease I (New England Biolabs) was used to catalyze the removal of excess oligo. The DNA-RNA hybrid was further purified using ChIP DNA clean \& Concentrator Kit (Zymo Research Corporation), RNaseH treated and circularized using CircLigase (Epicentre, Madison, WI). The libraries were amplified for 10 cycles using the following primers: $5^{\prime}$ AATGATACGGCGACCACCGACAGGTTCAGAGTTCTACAGTCCGACG-3' and ${ }^{\prime}$ ' CAAGCAGAAGACGGCATACGAGATXXXXXGTGACTGGAGTTCAGACGTGTGCTCTTCCG ATCT (barcode XXXXXX-underlined). The final product was ran on Novex $10 \%$-TBE gel, purified and cleaned-up as above. The libraries were sequenced on HiSeq 2000 (Illumina, San Diego, $\mathrm{CA})$ for 50 cycles according to the manufacturer's instructions (GeneCore, EMBL). Data analysis was performed using HOMER 4.3. Detailed instructions can be found at homer.salk.edu/homer (16). RNA-Seq was mapped using TopHat (v2.0.7) allowing up to two mismatches and reporting only one alignment for each read. Poor quality reads were filtered out (min. 97\% of bp over quality cutoff 10). Each sequencing experiment was normalized to a total of $10^{7}$ uniquely mapped tags and visualized by preparing custom tracks for the UCSC Genome browser. Differentially expressed genes were identified using edgeR (17). Following thresholds were used: P-value $<0.05$, RPKM $>0.5$ and fold change $>1.6$. Clustering results were generated by Cluster 3.0 and the output was viewed using Java Treeview. Clustering results were generated by Cluster 3.0 by normalizing and centering the gene expression tags to range from -1 to 1 . For gene ontology analysis DAVID Bioinformatics Resources 6.7, IPA and HOMER were used. The data is available in GEO under the accession number GSE58663 and can be accessed from ncbi.nlm.nih.gov/geo/query/acc.cgi?token=chkbssymbdqxbah\&acc=GSE58663.

\section{qRT-PCR}

Confluent cultures of HUVECs (3 donors) or HAECs were washed with PBS, followed by starvation of cells for $16 \mathrm{~h}$ with EGM medium supplemented with $0.5 \%$ FBS. For siRNA experiments, HUVECs were transfected with 10nM siRNA oligonucleotides using oligofectamine for $48 \mathrm{~h}$ (Life Technologies). siRNA efficiencies were detected by qRT-PCR (NR4A1 78.6\% $\pm 1.51 \%$, MET 77.4\%\% $\pm 0.99 \%$, MYCN $63.5 \% \pm 3.85 \%$, SNAI2 $73.3 \% \pm 2.08 \%$, VEGFR2 86.1\% $\pm 0.98 \%$; Supplemental Figure 1F) In all experiments, the cells were stimulated with VEGFs $(20,100,500 \mathrm{ng} / \mathrm{ml})$ or histamine $(0.2,2,20 \mu \mathrm{M})$ for 4 or $7 \mathrm{~h}$. In PI3K $\alpha / \delta$-inhibitor experiments, ECs were pre-treated for $30 \mathrm{~min}$ with $0.5 \mu \mathrm{M}$ Pictilisib (Sellekchem, Houston, TX) prior to VEGF or histamine stimulation for $7 \mathrm{~h}$. Tissue samples were collected from VEGF or histamine-treated mice as previously described and homogenized with PreCellys 24 tissue homogenizer (Bertin Technologies, Saint Quentin en Yvelines, France). RNA was extracted according to the manufacturer's instructions (Qiagen, Hilden, Germany). Total RNA was reverse transcribed into cDNA using random hexamers (Promega, Madison, WI) and RevertAID reverse transcriptase (MBI Fermentas, Hanover, MD). Quantitative measurements of mRNA levels were performed using the Assays-on-Demand gene 
expression products (Supplementary table 3) with StepOnePlus Real-Time PCR System (Applied Biosystems, Foster City, CA). Amplification of beta-2 microglobulin (B2M) was used as an endogenous control with human endothelial cells and peptidylprolyl osomerase $A$ (PPIA) and GADPH with mouse tissues.

\section{Western blot}

Tissues were homogenized in lysis buffer [50 mM Tris- $\mathrm{HCl}, \mathrm{pH} 7.5,150 \mathrm{mM} \mathrm{NaCl}, 1 \mathrm{mM}$ EDTA, $1 \%$ Triton X-100, $0.5 \%$ sodium deoxycholate, $0.1 \%$ SDS, $10 \%$ glycerol, PhosSTOP and protease inhibitor tablets (Roche Applied Science, Penzberg, Germany)] with Precellys Lysing kit (Bertin Technologies). Protein concentrations were determined by BCA Protein Assay Kit (Thermo Fisher Scientific). Proteins were separated using SDS-PAGE, followed by transfer to nitrocellulose membranes (Pure Nitrocellulose Membrane, Bio-Rad). Membranes were blocked in 5\% w/v BSA in TBS and 0.1\% Tween 20 and incubated with primary antibodies MYCN (Abnova, Taipei, Taiwan), VEGF (Santa Cruz Biotechnology, Dallas, TX) and $\beta$-Actin (Cell Signaling Technology, Danvers, MA) overnight at $4^{\circ} \mathrm{C}$. Blots were visualized using HRP-conjugated secondary antibodies and ECL 2 Western Blotting Substrate (Thermo Fisher Scientific) and detected with Typhoon 9400 (GE Heathcare, Little Chalfont, UK) scanner.

MTS assay. HUVECs were seeded on 96-well plates at 4.000 cells/well and transfected with $10 \mathrm{nM}$ siRNAs for $48 \mathrm{hrs}$ or treated with $0.5 \mu \mathrm{M} \mathrm{PI} 3 \mathrm{~K} \alpha / \delta$-inhibitor for $8 \mathrm{hrs}$. Cell viability was measured with MTS-reagent (Promega) according to the manufacturer's instructions using absorbance of $492 \mathrm{~nm}$. No cytotoxicity was detected with $\mathrm{PI} 3 \mathrm{~K} \alpha / \delta$-inhibitor in comparison to untreated control cells (data not shown).

\section{Statistical analysis}

Data are presented as mean \pm SEM. Following Shapiro-Wilk test for normal distribution, the groups were compared with Mann-Whitney rank-sum test or One-way ANOVA followed by Dunnett's multiple comparison test. (GraphPad Prism4, La Jolla, CA, USA). P<0.05 was used to define statistical significance. 


\section{RESULTS}

\section{Common gene expression profiles regulate VEGF and histamine induced changes in EC function}

To characterize the transcriptional alterations of genes known to regulate angiogenic signaling and to compare the molecular mechanisms induced by histamine and VEGF, RNAseq was performed from primary human vein endothelial cells (HUVEC) after $7 \mathrm{~h}$ of exposure to histamine or VEGF. Fold changes of several genes known to regulate cell adhesion, cell proliferation, ion flux and immune response were compared between the factors in ECs. 76 genes were shown to be commonly regulated between VEGF- $A_{165}$ and histamine (Figure $1 \mathrm{~A}$, B; Table 1, Supplementary Table 1), representing $~ 53 \%$ of all VEGF-regulated transcripts and $\sim 26 \%$ of all histamine-regulated transcripts. Accordingly, $47 \%$ of all RNA transcripts $(n=67)$ were solely regulated by VEGF and $\sim 74 \%$ by histamine $(n=215$; Table 2$)$. Within the commonly induced genes a similar regulation pattern was observed, having strong positive correlation ( $r^{2}=0.8$; Figure 1C). Gene ontology (GO) and KEGG analyses demonstrated that the commonly regulated genes were involved in cell differentiation, development and cell adhesion (Figure 1D, F-G; Table 3). Both factors regulated G-protein coupled receptor signaling (GPCR) involved in calcium influx/release, cytokine-cytokine interactions as well as PI3K-Akt pathway involved e.g. in cell proliferation (Figure 1E, Table 4, Supplementary Table 2). Histamine also regulated genes involved in granulocyte/agranulocyte diapedesis, type I interferon pathway, as well as in chemokine and Toll-like receptor signaling pathways. Additionally, activation of genes involved in complement and coagulation cascades, such as plasminogen activators, were detected. To exclude that histamine did not induce VEGF expression and thereby a similar expression pattern of commonly regulated genes, RT-qPCR and Western Blot (WB) analyses were performed from histamine-stimulated cells from $4 \mathrm{~h}$ and $7 \mathrm{~h}$ time points for RT-qPCR and 7h for WB. No significant change in VEGF expression was detected either in RT-qPCR experiments, RNA-seq data or in WB (data not shown, Supplementary Table 1, Supplemental Figure 1A). Additionally, no changes in expression of VEGFRs were detected by RNA-seq.
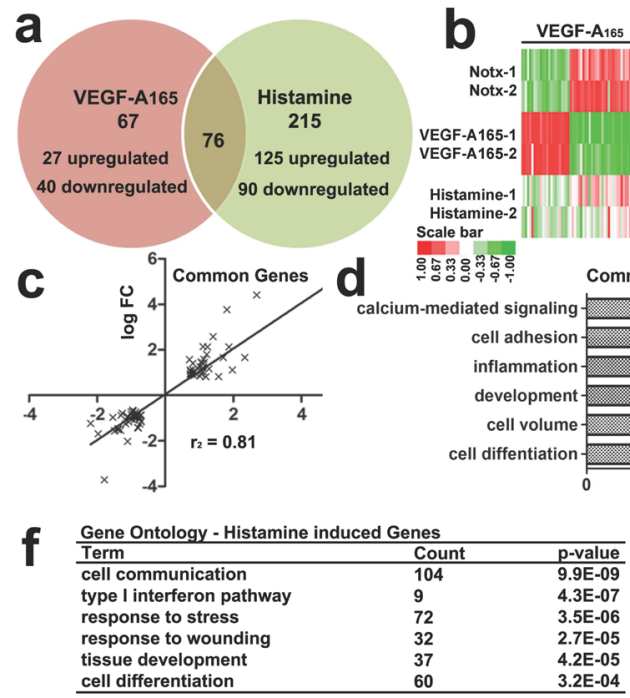

\begin{tabular}{lll}
\multicolumn{4}{l}{ Gene Ontology - Histamine induced Genes } \\
\hline Term & Count & p-value \\
\hline cell communication & 104 & $9.9 \mathrm{E}-09$ \\
type I interferon pathway & 9 & $4.3 \mathrm{E}-07$ \\
response to stress & 72 & $3.5 \mathrm{E}-06$ \\
response to wounding & 32 & $2.7 \mathrm{E}-05$ \\
tissue development & 37 & $4.2 \mathrm{E}-05$ \\
cell differentiation & 60 & $3.2 \mathrm{E}-04$ \\
\hline
\end{tabular}

Q \begin{tabular}{lll} 
Gene Ontology - VEGF Induced Genes & & \\
\hline Term & Count & p-value \\
\hline regulation of cell differentiation & 12 & $1.6 \mathrm{E}-03$ \\
system, organ development & 22 & $8.5 \mathrm{E}-03$ \\
response to stress & 19 & $1.5 \mathrm{E}-02$ \\
response to wounding & 9 & $1.5 \mathrm{E}-02$ \\
cell communication & 25 & $1.9 \mathrm{E}-02$ \\
\hline
\end{tabular}


Figure 1. Histamine and VEGF Regulate Gene Expression Similarly and Induce Angiogenic Signals in Endothelial Cells

(A) RNA-sequencing was performed from HUVECs treated with VEGF-A165 or histamine for 7h. Venn diagram showing all significantly regulated genes in treated ECs, revealing 76 commonly regulated genes. (B) Heat map showing all genes in non-treated ECs (Notx) and in VEGF or histamine treated ECs. Normalized gene expression values are shown (red, upregulated; green, downregulated genes). (C-D) Scatter plot (C) and gene ontology analysis (D) of commonly regulated genes. (E) KEGG pathway analysis of commonly regulated genes (grey), histamine (black) and VEGF (white) regulated genes. (F-G) Gene ontology analyses of histamine (F) and VEGF regulated genes (G). In all data, two independent experiments were performed.

\section{VEGF-mediated short-term vascular hyper-permeability is time-dependent and varies in different vascular beds according to used VEGF family member}

To validate the role of VEGF and histamine in remodeling cell-cell adhesion after both short (10-30min post transduction, p.t.) and long-term exposure (after $7 \mathrm{~h}$ p.t.), endothelial cell (EC) permeability assays, immunostaining of cell junctional proteins and EC tube formation assays were performed. First, vascular hyper-permeability response induced by histamine and VEGFR2-binding VEGF family members was compared in the intact vasculature of C57/BI6 mice by laser scanning microscopy. Extravasation of green fluorescent microspheres indicated vascular permeability. After 10 or $30 \mathrm{~min}$ of injection of permeabilizing agents and fluorescent microspheres (i.v.), mice were sacrificed, perfused and the vasculature was immunostained by CD31 antibody. Vascular leakage of $200 \mathrm{~nm}$ fluorescent microspheres was visible in $20 \mu \mathrm{m}$-sized vessels of trachea with HR1/2-binding histamine, VEGFR1/2-binding VEGF- $A_{165}$ and VEGFR2-binding VEGF-F after $10 \mathrm{~min}$ of exposure. This indicated a fast hyper-permeability response of the vessels (Figure 2A) probably due to gap formations between ECs. No vascular leakage was detected after injection of VEGFR2/3-binding VEGF-D $D_{\triangle N \Delta C}$ or saline injected control mice without permeabilizing agents. Quantification of fluorescent microspheres from CD31-labelled vessels showed a 49-fold increase of vascular permeability in histamine and a 8-fold increase in VEGF-F-treated mice (Figure $2 B, C$ ), whereas no statistically significant vascular leakage was detected with VEGF-A $A_{165}$ after 10 min of exposure (1.6-fold, $P>0.05$ ). Instead, 30 min after injection a 17 -fold increase in vascular permeability was detected with VEGF$\mathrm{A}_{165}$ and a 12 -fold with VEGF-F (Figure $2 \mathrm{H}$ ), demonstrating that vascular hyper-permeability induced by VEGFs increased over time. No differences between treatments were detected in liver (10 min or 30 min; Figure 2D), whereas VEGF-F caused a 5-fold less vascular leakage in spleen at $10 \mathrm{~min}$ time point compared to other VEGFs or histamine (Figure 2E). Additionally, no leakage of microspheres was detected in kidneys or heart in any of the time points (data not shown). To further distinguish the role of VEGF co-receptors NRP1/2 and HSPGs in VEGF-induced vascular hyper-permeability, experiments were performed with VEGF isoform $A_{121}$ which has reduced binding affinity to NRP receptors and HSPGs compared to VEGF-A $A_{165}$ or VEGF-F and increased solubility (12). At 30 min time point, VEGF-A $A_{121}$ did not induce statistically significant vascular permeability in trachea (Figure $2 F, H$ ), whereas in the lungs and spleen, a 2-fold increase in vascular leakage was detected (Figure $2 \mathrm{I}, \mathrm{J}$ ). Therefore, VEGF binding to NRP-receptors or HSPGs seem to further affect the permeability profile induced by VEGFs. In accordance with in vivo results, in vitro permeability assay in HUVEC cells showed that VEGF- $A_{165}$ significantly induced EC permeability of fluorescent marker (Lucifer yellow) after 15 min of exposure, whereas VEGF-A $A_{121}$ or VEGF-D $D_{\Delta N \Delta C}$ had no effect (Supplemental Figure 1B). Redistribution of VE-cadherin, a major adherens junction (AJ) 
protein, and changes in actin organization were also seen $30 \mathrm{~min}$ after exposure to VEGF$A_{121}, V E G F-A_{165}, V E G F-F$ and histamine, whereas VEGF- $D_{\triangle N} \Delta C$ did not induce any major changes (Figure 3B).
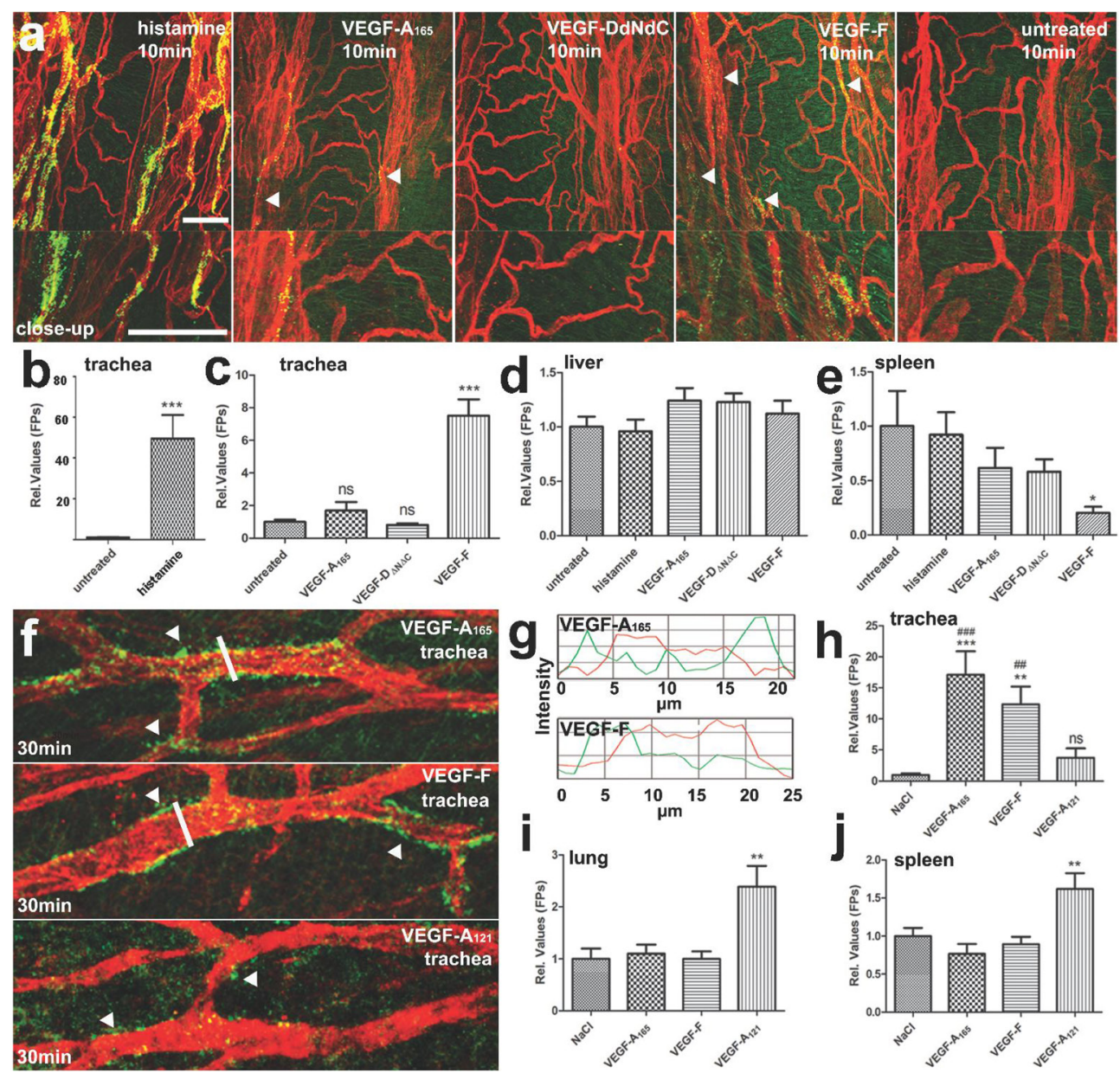

Figure 2. Tissue Specific and Time-Dependent Vascular Hyper-permeability in Intact Vasculature of Mice after Short-term Induction by Histamine and VEGFs Binding to VEGFR2/NRP

(A) Tracheal whole mounts labeled with endothelium marker CD31 (red). Intravenous administration of histamine or various VEGFs together with 200nm-fluorescent microspheres (green) in C57/BI6 mice at 10 min after exposure. $\mathrm{NaCl}$ was used as a negative control together with fluorescent microspheres (untreated). Scale bar, 100 $\mu \mathrm{m}$. (B-E) Quantification of the vascular leakage from trachea (B, C), liver (D) and spleen (E) at 10 min after exposure ( $n=4$ mice/group). (F, G) Close-up and plot profile views of the $20 \mu \mathrm{m}$-sized leaky vessels are presented after $30 \mathrm{~min}$ of exposure to VEGFs, showing fluorescent microspheres (green line) outside of the vessels (red line). White bars (F) represent the site from which the corresponding intensity plot profile area is derived (G). (H-J) Quantification of vascular leakage from trachea $(\mathbf{H})$, lung $(\mathbf{I})$ and spleen $(\mathrm{J})$ at $30 \mathrm{~min}$ after VEGF exposure ( $\mathrm{n}=4 \mathrm{mice} / \mathrm{group})$. In all data, mean+SEM presented. P-values: $<0.05^{*},<0.01^{* *},<0.001^{* * *}$.

\section{VEGF regulates gene expression of a major tight junction protein claudin-5 via SNAI2}

Although short-term exposure to permeabilizing agents has been shown to induce phosphorylation cascades e.g. in VE-cadherin affecting formation of AJs, changes in EC gene 
expression leading to angiogenic processes are not well known. RNA-seq from HUVECs at $7 \mathrm{~h}$ after exposure revealed that histamine and VEGF both regulate genes involved in AJ and tight junction (TJ) cell-cell adhesion modulation as well as re-organization of actin filaments that mediate gap formation between ECs (Figure 3A). Both factors downregulated the major TJ protein claudin-5 (CLDN5), whereas no change in the expression of the major AJ protein VE-cadherin (CDH5) was detected. To further validate the VEGF induced effects, other VEGF family members were studied. RT-qPCR experiments confirmed the significant downregulation of CLDN5 in both venous (HUVEC) and aortic (HAEC) primary human ECS after $4 \mathrm{~h}$ and $7 \mathrm{~h}$ of exposure by VEGF- $\mathrm{A}_{121}$, VEGF- $\mathrm{A}_{165}$, VEGF-F and histamine, whereas VEGF$D_{\triangle N \Delta C}$ had no effect. (Figure $3 C$, data not shown). This implicates that high affinity binding of VEGF to VEGFR2 regulates CLDN5 expression and thereby cell-cell adhesion. Similarly, a trend in CLDN5 downregulation was detected in lung tissue of C57/BI6 mice after 4h of exposure (i.v.) to VEGF-A $A_{165}$, VEGF-F or histamine, however the effect was not statistically significant (Supplemental Figure 1C). In addition to downregulation of CLDN5 by both VEGF and histamine, both factors regulated several other junctional molecules independently. By RNA-seq, VEGF was shown to induce downregulation of other cell-cell adhesion genes such as immunoglobulin superfamily cell adhesion receptors (AMIGO3, CADM4) and junction adhesion molecule 2 (JAM2). Histamine instead regulated other genes associated with TJs e.g. claudin superfamily members (CLDN1, CLDN14), coxsackie virus and adenovirus receptor (CXADR), cingulin-like 1 (CGNL1), membrane associated guanylate kinase CNKSR and membrane protein MPP7. Altogether, the presented data indicate that besides internalization of VE-cadherin due to well-known phosphorylation cascades induced by VEGF or histamine and thereby vascular hyper-permeability, cell junction formation is regulated by complex cellular mechanisms at the gene expression level affecting particularly TJ formation.

Besides cell junction molecules, SNAI2 expression was shown to be upregulated 2.8-fold in VEGF-treated ECS $(P<0.01)$ while transcript levels were unchanged in histamine-treated cells. SNAI2, a zinc finger protein, has been previously suggested to act as a transcriptional regulator of claudins in keratinocytes and to enhance cancer cell migration. To distinguish the role of SNAI2 and VEGFR2 signaling in potentially regulating CLDN5 expression and EC migration in our study, siRNA experiments against VEGFR2 and SNAI2 were performed in HUVECs. In VEGFR2 siRNA treated ECs, histamine but not VEGF was able to downregulate CLDN5 expression (Figure 3D). Similarly, silencing of SNAI2 was able to inhibit VEGFmediated CLDN5 downregulation, but not histamine-induced effects (Figure 3E). Therefore, VEGFR2 and SNAI2 seem to specifically regulate VEGF-mediated TJ assembly. To detect the role of SNAI2 in angiogenesis and cell proliferation, matrigel-based in vitro tube forming and cell viability assays were performed in HUVECs. In comparison to control siRNA treated cells, tube formation of ECs was inhibited in siSNAI2-treated cells (48h), indicating a role for SNAI2 in regulating vessel formation (Figure3F-H). Stimulation with VEGF (7h) further decreased EC tube formation in siSNAI2 treated ECs and induced the formation of cell clusters with abnormal morphology. Additionally, reorganization of F-actin filaments, known to be associated with formation of TJs, was observed in siSNAI2-treated ECs (Supplemental Figure 1J). siSNAI2 also reduced EC proliferation compared to control siRNA treated ECs (Figure $4 \mathrm{~L})$. Thus, SNAI2 seems to be an important regulator of cell adhesion, EC tube formation and TJ assembly in VEGF activated ECs but not by histamine. 


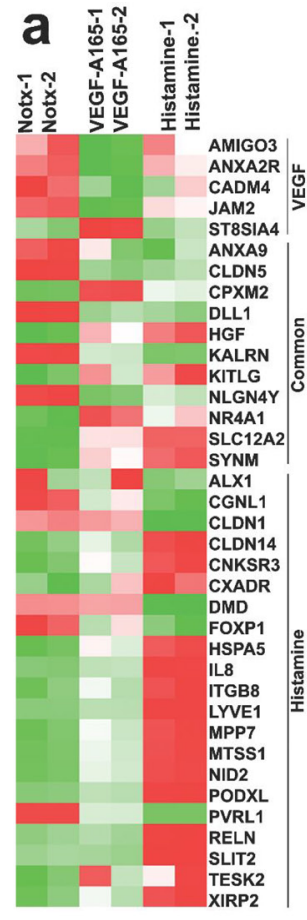

Cell-Cell Adhesion
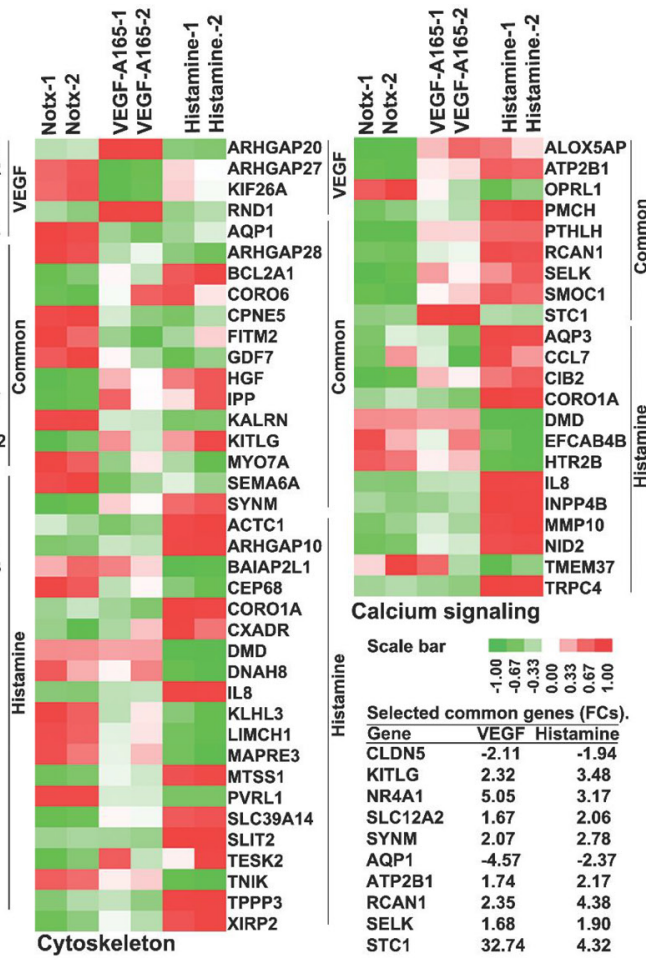

Calcium signaling

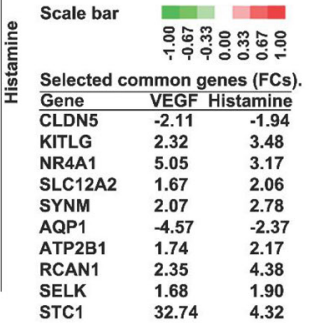

b

F-actin

DAPI
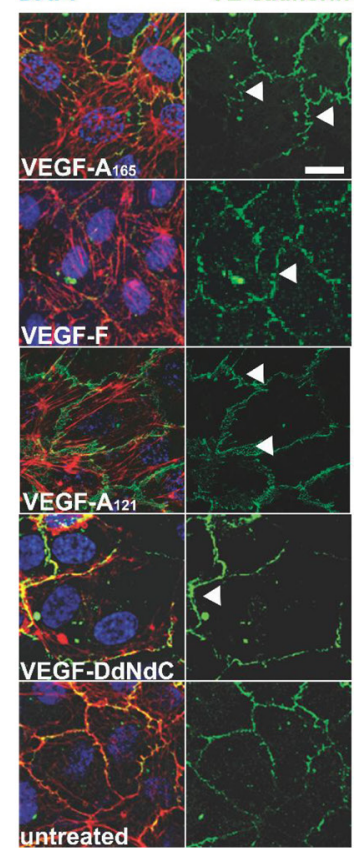

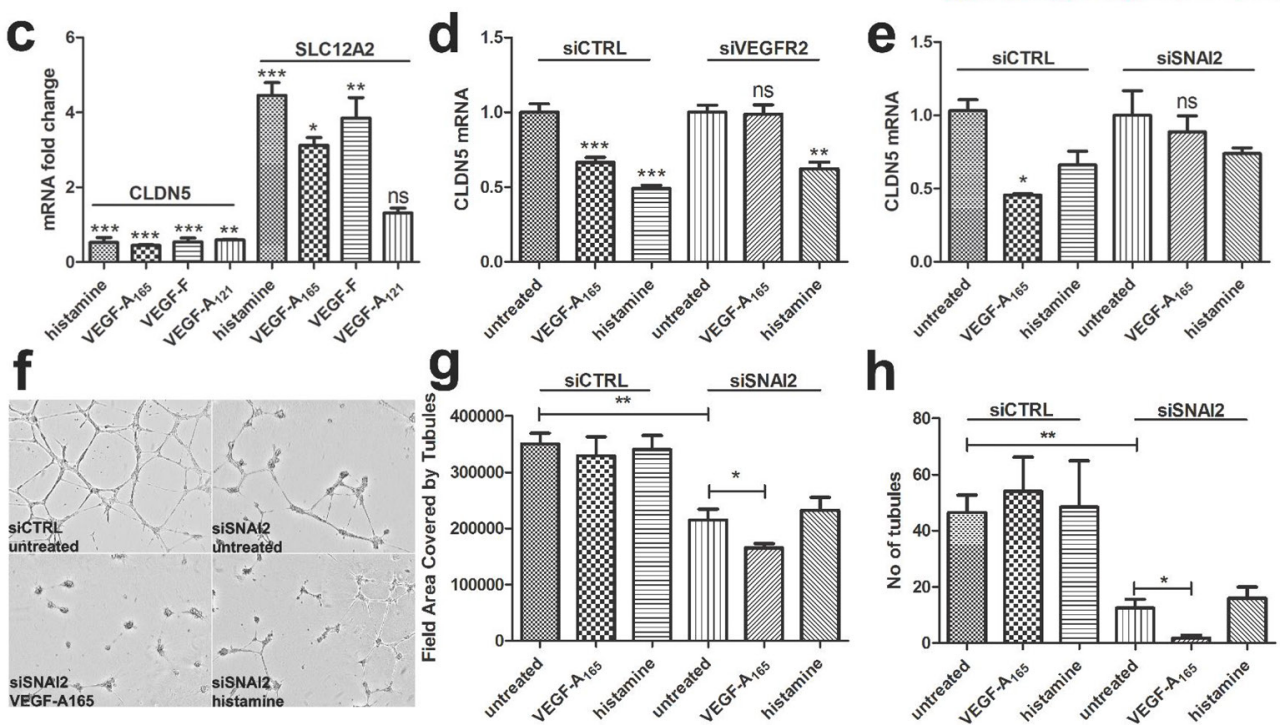

Figure 3. Regulation of Cell-Cell Adhesion and Calcium Signaling by Histamine and VEGFs by Inducing Changes in Gene Expression

(A) Heat map showing genes in cell-cell adhesion, cytoskeleton and calcium signaling after VEGF and histamine stimulation for 7h in HUVEC cells by RNA-sequencing. Normalized gene expression values are shown (red, upregulated; green, downregulated genes). In all data, two independent experiments were performed. Fold changes (FC) of selected commonly regulated genes are shown in the table. (B) VE-cadherin (green) redistribution 
(arrow heads) and F-actin mediated stress fiber formation (red) after VEGF treatment for 30 min. VEGF-A165 and untreated cells were used as positive and negative controls. DAPI (blue) was used for staining nuclei. No changes were detected with VEGF-D $\Delta N \Delta C$. Scale bar, 20 $\mu$ m. (C) mRNA expression levels of claudin-5 (CLDN5) and solute carrier family member SLC12A2 after 7h stimulation with histamine or VEGFs in HUVECs detected by qRT-PCR. (DE) Role of VEGFR2 signaling in regulating CLDN5 expression. CLDN5 mRNA expression levels in VEGF receptor 2 (siVEGFR2; D), Zinc finger protein SNAI2 (siSNAI2; E) or control (siCTRL) siRNA silenced HUVECs after 7h stimulation with histamine or VEGF-A detected by qRT-PCR. (F) In vitro tube formation assay in HUVEC cells after $48 \mathrm{~h}$ siRNA treatment with siSNAI2 and siCTRL and 7h stimulation with histamine or VEGF. (G-H) In vitro tube formation assay in HUVEC cells after $48 \mathrm{~h}$ siRNA treatment with siSNAI2 and $7 \mathrm{~h}$ stimulation with VEGF. Field area and number of tubules were detected by Angiosys program (4 images/treatment). In all data, mean+SEM presented. P-values: $<0.05^{*},<0.01 * *,<0.001 * * *$.

\section{TJ formation is regulated via PI3K-signaling by VEGF}

Regulation of extracellular matrix (ECM) organization and EC proliferation by cytokines, growth factors and transcription factors have been shown to mediate angiogenic processes, including EC-EC adhesion and CLDN5 expression. In this study, 18 transcripts regulating extracellular matrix (ECM) remodeling were detected by RNA-seq in histamine-treated ECs (7h, HUVEC, Figure 4A). Histamine also modulated matrix metalloproteinases (MMP), which are crucial in angiogenesis associated cell migration, e.g. MMP10, MMP19, as well as a serine protease reelin (RELN), whereas in VEGF treated cells the expression levels of ADAM metallopeptidase (ADAMTS10) and MMP28 were altered. Histamine and VEGF also shared 14 commonly regulated genes involved in cell proliferation, such as hepatocyte growth factor (HGF; Figure 4B, Table 3). Besides these, VEGF upregulated platelet derived growth factor D (PDGFD, Figure 4B), whereas macrophage chemotactic factors chemokine ligand 7 (CCL7) and interleukin-8 (IL8) were only significantly upregulated in histamine-stimulated cells. Since HGF has previously been connected with the regulation of TJ formation likely therefore influencing CLDN5 expression, and angiogenic signaling by PI3K-Akt pathway, it was studied in greater detail. First, WB analysis of HGF-stimulated ECs showed that there was no increase in the expression level of VEGF protein (Supplemental Figure 1A) that could explain detected CLDN5 downregulation and induced cell proliferation by histamine and VEGF via indirect mechanism. This was further confirmed by siRNA experiments against HGF receptor MET (siMET) that showed no inhibition of CLDN5 downregulation after stimulation with VEGF or histamine (Figure 4E). Further PI3K inhibitor experiments in ECs revealed that upregulation of HGF was inhibited by both VEGF and histamine (7h; Figure 4D), whereas CLDN5 downregulation was only inhibited in VEGF stimulated PI3K inhibitor-treated ECS (Figure 4F). Thus, although the PI3K-Akt pathway is required for VEGF and histamine induced HGF mRNA upregulation, HGF is not the common mediator required for regulating CLDN5 expression and subsequent TJ formation. Interestingly, PI3K-signalling was required particularly for VEGF induced CLDN5 downregulation, whereas no effect was observed with histamine. This implies that histamine and VEGF regulate TJ formation via complex and partially different cellular mechanisms involving multiple cellular factors affecting to EC-EC adhesion and cell proliferation. 


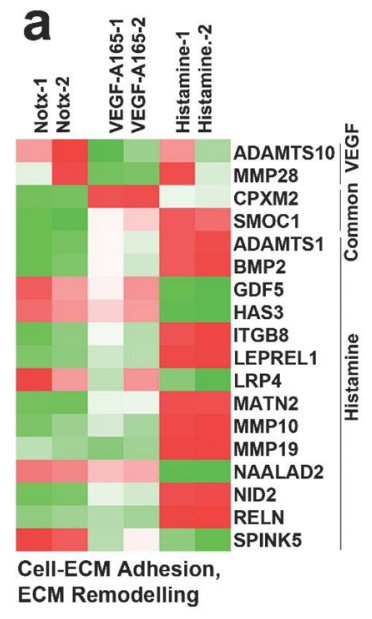

\section{Scale bar

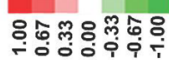

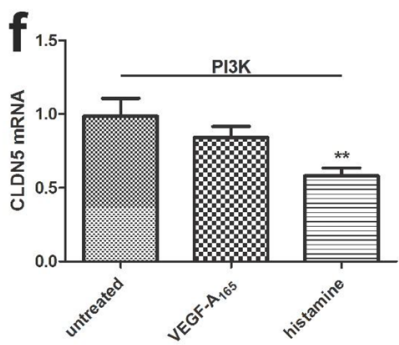

i

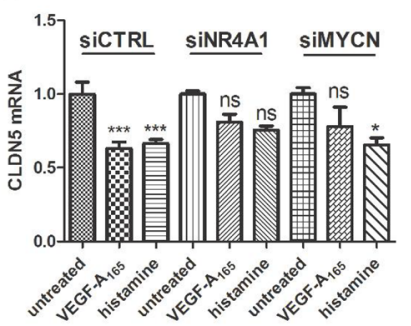

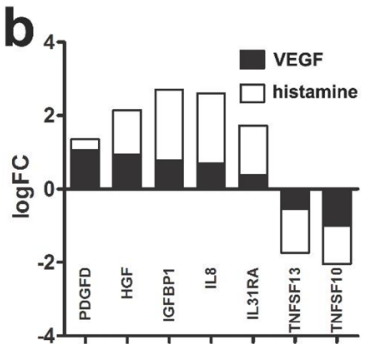

Gene Symbol
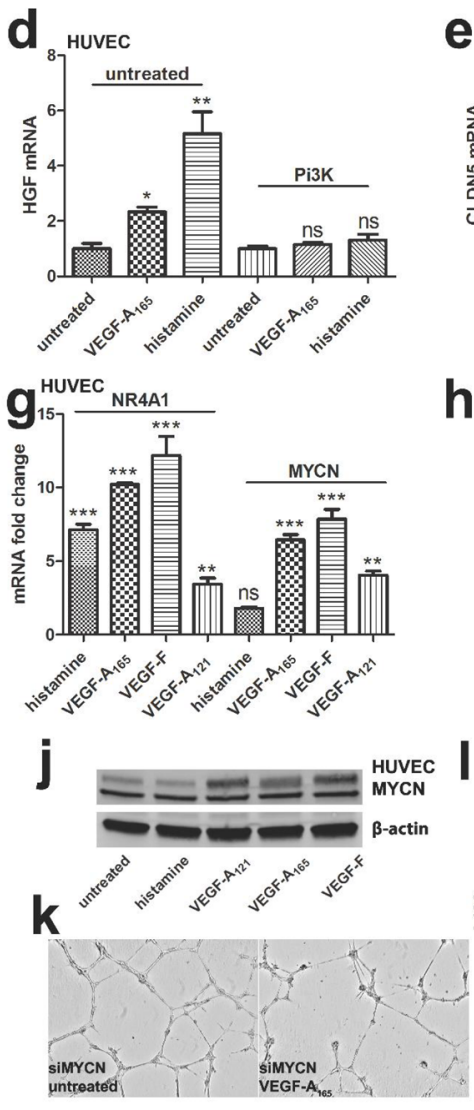

e

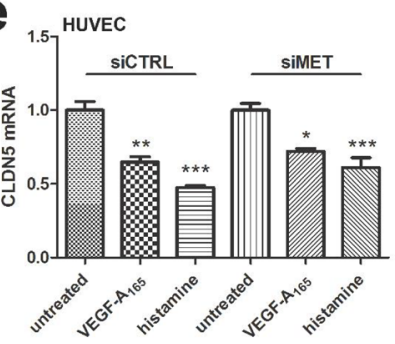

$\mathrm{h}$

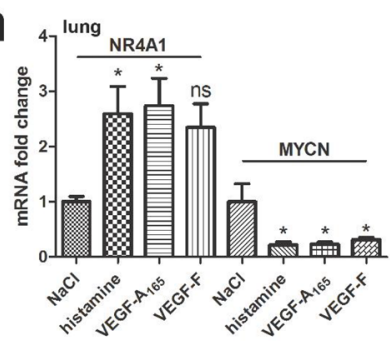

Figure 4. Regulation of Endothelial Cell Proliferation by Histamine and VEGFs

(A) Heat map showing genes involved in cell-ECM adhesion regulated by VEGF and histamine stimulation in HUVECS at $7 \mathrm{~h}$ by RNA-sequencing. In all data, two independent experiments were performed. Normalized gene expression values are shown (red, upregulated; green, downregulated genes). (B-C) Genes involved in the regulation of growth factors, cytokines (B) and transcriptional activity (C) by VEGF and histamine. Log fold changes are shown. (D) PI3KAkt pathway is required for HGF upregulation. HGF mRNA expression levels in untreated and PI3K inhibitor treated HUVECs after histamine and VEGF stimulation for 7h detected by qRT-PCR. (E) HGF-MET signaling does not regulate CLDN5 expression. CLDN5 mRNA expression levels in MET (siMET) or control (siCTRL) siRNA treated HUVECs after 7h stimulation with histamine or VEGF-A detected by qRT-PCR. (F) PI3K-Akt pathway is required for CLDN5 downregulation by VEGF. CLDN5 mRNA expression levels in untreated and PI3K inhibitor treated HUVECs after histamine and VEGF stimulation for 7h detected by qRT-PCR. (G) Upregulation of transcription factors NR4A1 and MYCN in HUVECs after histamine and VEGF stimulation for 7h detected by qRT-PCR. (H) Upregulation of NR4A1 mRNA expression and downregulation of MYCN in lung tissue by histamine and VEGFs after 4 h of systemic delivery 
detected by qRT-PCR. (I) NR4A1 regulates TJ formation. CLDN5 mRNA expression levels in NR4A1 (siNR4A1), MYCN ( SiMYCN) or siCTRL siRNA treated HUVECs after 7h stimulation with histamine or VEGF detected by qRT-PCR. (J) MYCN protein expression is upregulated by VEGFs, but not by histamine in HUVECs after 7h of treatment detected by WB. (K) In vitro tube formation assay in HUVEC cells after $48 \mathrm{~h}$ siRNA treatment with siMYCN and $7 \mathrm{~h}$ stimulation with VEGF. Representative images are shown. (L) HUVEC proliferation/survival was decreased by siRNAs against MYCN, NR4A1 and SNAI2 detected by MTS assay at $48 \mathrm{~h}$ time point. In all data, mean+SEM presented. P-values: $<0.05^{*},<0.01 * *,<0.001 * * *$.

\section{VEGF and histamine regulate expression of transcription factors RCAN1, NR4A1 and MYCN that induce EC proliferation}

In addition to cytokines and growth factors, transcription factors (TFs) are crucial in regulating cell adhesion, proliferation and angiogenesis. To identify other TFs than SNAI2 that are required for cell adhesion/proliferation and regulation of TJ formation, this was studied further. NR4A1, RCAN1 and MAFB were found to be regulated by both VEGF and histamine by RNA-seq (7h, HUVEC). Besides these, 13 other TFs were specifically regulated by histamine and 8 TFs by VEGF (Figure 4C). Of all the TFs, RCAN1, NR4A1 and MYCN were the most highly upregulated and thus chosen for further functional studies. In addition to RCAN1, a regulator of calcium-dependent calcineurin 1, both histamine and VEGF upregulated several genes that are activated in ECs due to overload of calcium, such as plasma membrane calcium transporting ATPase 1 (ATP2B1; Supplemental Figure 1D), stanniocalcin-1 (STC-1; Supplemental Figure 1E) and aquaporin 1 (AQP1, data not shown). Histamine also upregulated expression of $\mathrm{Ca}^{2+}$-influx channel TRPC4 and $\mathrm{Ca}^{2+}$-activated chloride channel anoctamin 1 (ANO1) (Supplemental Figure 1D, data not shown). STC-1 and RCAN1 were also upregulated after treatment of ECs (48h) with adenovirus vector expressing VEGF- $\mathrm{A}_{165}$ (data not shown). To elaborate more the role of VEGFRs in calcium signaling further, RCAN1 expression was detected after stimulation with various VEGFR2 binding VEGFs and in VEGFR2 siRNA-treated HUVECs. In ECs treated with siVEGFR2, histamine but not VEGF was able to upregulate RCAN1 expression (Supplemental Figure $1 \mathrm{G})$, indicating that both factors regulate RCAN1 regardless of their distinct receptor binding profiles. Interestingly, no significant changes were found in the expression levels of RCAN1, STC1, ATP2B1 or a mediator of sodium/chloride transporter SLC12A2 by VEGF-A $A_{121}$ (Figure 3C, Supplemental Figure 1D-E). VEGF-F, a selective ligand of VEGFR2, instead seemed to induce a similar expression pattern of calcium-induced genes as VEGF-A $\mathrm{A}_{165}$. Since VEGFR2 binding affinities of VEGF- $A_{121}$ and VEGF- $A_{165}$ are similar, NRP/HSPG co-receptor binding may play a role in VEGF-induced calcium signaling and gene regulation.

Besides RCAN1, upregulation of NR4A1 expression, an orphan nuclear superfamily member involved in angiogenesis and inflammation, was observed in ECs (7h HUVEC) and lung tissue of C57/Bl6 mice (i.v.; 4h) by VEGFR2 binding VEGFs and histamine (Figure 4G, H). In comparison, MYCN gene expression, linked with regulation of cell proliferation e.g. in neuroblastoma, was upregulated by all VEGFs but not by histamine (7h, HUVEC; Figure 4G, J). Both NR4A1 and MYCN were also upregulated after longer treatment of ECs (48h) with adenovirus vector expressing VEGF-A $\mathrm{A}_{165}$ (data not shown). A different role of MYCN in ECS compared to lung tissue was seen after systemic delivery of VEGF or histamine in C57/BI6 mice, as MYCN was downregulated (i.v., 4h; Figure 4H). The importance of NR4A1 and MYCN in EC function was further analyzed in a cell proliferation/survival assay in HUVECs, which demonstrated a significant decrease in cell viability after siRNA treatment against NR4A1 and MYCN (48h, Figure 4I). In siNR4A1 treated ECs, downregulation of CLDN5 was 
inhibited after VEGF and histamine treatment (7h), showing that NR4A1 affects TJ regulation, but is not specific for VEGF (Figure 4I). In siVEGFR2 experiments, NR4A1 upregulation was further shown to be independent on VEGFR2 expression (Supplemental Figure $1 \mathrm{H}$ ). This indicates that an indirect mechanism, independent of VEGFR2 signaling induces upregulation of this TF after stimulation by histamine and VEGF. Instead, treatment of ECs with siMYCN inhibited only VEGF-induced CLDN5 downregulation, whereas no effect was observed in histamine-induced ECs. Interestingly, no statistically significant differences were found in EC tube formation with either siMYCN or siNR4A1 treated ECs (Figure 4K, Supplemental Figure 1I), confirming earlier observations that they mainly affect EC proliferation. To conclude, multiple TFs regulate TJ formation and cell adhesion affecting cell proliferation. For VEGF, TFs SNAI2, NR4A1 and MYCN are important regulators of CLDN5 expression and subsequent TJ formation, whereas histamine is regulated only by NR4A1. Altogether, these data demonstrate that both VEGF and histamine regulate genes involved in cell adhesion, calcium signaling and cell proliferation affecting angiogenic signaling of ECs (Figure 5). Since the VEGFR2 binding ligands VEGF-A $A_{165}$ and VEGF-F induce similar EC responses, binding efficiency to VEGFR2 likely determines the vascular/EC hyperpermeability response. Common TFs, such as NR4A1 and RCAN1 regulate similar signaling mechanisms of histamine and VEGF. CLDN5 expression and thus TJ formation and EC-EC adhesion is regulated differently between histamine and VEGF and involves signaling via PI3K, SNAI2, NR4A1 and MYCN. 


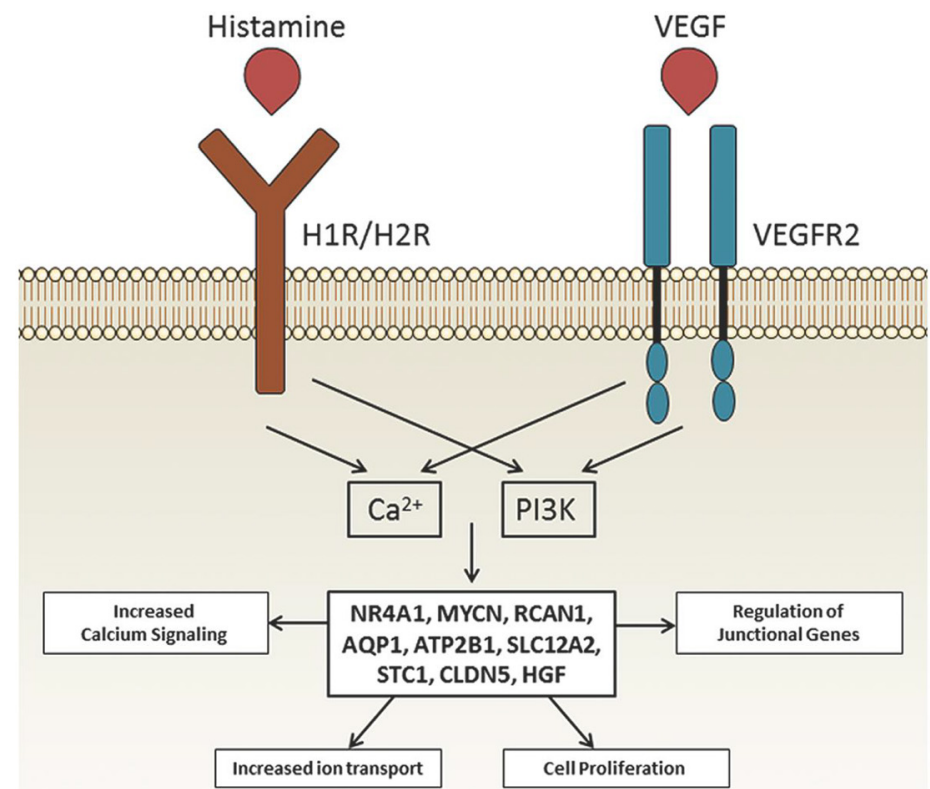

Figure 5. Common Gene Regulation by Histamine and VEGF leads to Angiogenic Processes in Endothelial Cells Common molecular mechanisms, such as PI3K-Akt pathway and calcium signaling exist between histamine ad VEGF despite of their distinct receptor binding profiles to H1R/H2R and VEGFRs, respectively. Selected genes affecting EC proliferation, cell-cell adhesion and ion flux are shown.

Table 1. Top 10 commonly upregulated genes after VEGF or histamine stimulation in HUVECs at 7h time point.

\begin{tabular}{lllll}
\hline Gene Symbol & $\begin{array}{l}\text { LogFC } \\
\text { (VEGF) }\end{array}$ & $\begin{array}{l}\text { FDR } \\
\text { (VEGF) }\end{array}$ & $\begin{array}{l}\text { LogFC } \\
\text { (Histamine) }\end{array}$ & FDR (Hista \\
\hline Commonly Regulated Genes: & & & & \\
STC1 & & & & \\
PMCH & 5.03 & $2.11 \mathrm{E}-129$ & 2.11 & $2.74 \mathrm{E}-09$ \\
NR4A1 & 2.69 & $3.33 \mathrm{E}-05$ & 4.41 & $8.77 \mathrm{E}-31$ \\
A2M & 2.34 & $5.15 \mathrm{E}-18$ & 1.66 & $1.20 \mathrm{E}-07$ \\
CCL20 & 1.98 & $4.15 \mathrm{E}-13$ & 1.11 & $3.04 \mathrm{E}-03$ \\
DNALI1 & 1.81 & $3.99 \mathrm{E}-02$ & 3.77 & $2.18 \mathrm{E}-16$ \\
LBH & 1.70 & $1.81 \mathrm{E}-12$ & 1.67 & $1.93 \mathrm{E}-13$ \\
RCAN1* & 1.40 & $4.04 \mathrm{E}-12$ & 2.58 & $1.25 \mathrm{E}-55$ \\
KITLG & 1.23 & $9.17 \mathrm{E}-26$ & 2.13 & $5.14 \mathrm{E}-77$ \\
HDAC9 & 1.21 & $2.11 \mathrm{E}-18$ & 1.80 & $3.80 \mathrm{E}-27$ \\
& 1.14 & $1.16 \mathrm{E}-03$ & 1.59 & $3.00 \mathrm{E}-08$
\end{tabular}

Abbreviations: STC1 stanniocalcin 1, PMCH pro-melanin-concentrating hormone, NR4A1 nuclear receptor subfamily 4, group A, member 1, A2M alpha-2-macroglobulin, CCL20 chemokine (C-C motif) ligand 20, DNALI1 dynein, axonemal, light intermediate chain 1; LBH limb bud and heart development, RCAN1 regulator of calcineurin 1, HDAC9 histone deacetylase $9 .{ }^{*}$ Commonly upregulated transcription factors. 
Table 2. Top 10 VEGF or histamine-specific genes upregulated in HUVECs at $7 \mathrm{~h}$ time point. Gene Symbol

VEGF-specific:
MYCN*
EGR3*
SNAI2*
ARHGAP20
PRDM1
DNAJB9
MEF2C*
ST8SIA4
PDGFD
SULT1C4

v-myc myelocytomatosis viral related oncogene

$2.1 \mathrm{E}-129$

early growth response 3

6.07E-31

snail homolog 2

3.77E-30

Rho GTPase activating protein 20

3.66E-29

PR domain containing 1 , with ZNF domain

9.17E-26

DnaJ (Hsp40) homolog, subfamily B, member 9

4.35E-20

myocyte enhancer factor $2 \mathrm{C}$

2.11E-18

ST8 alpha-N-acetyl-neuraminide alpha-2,8-sialyltransferase 4

$5.15 \mathrm{E}-18$

platelet derived growth factor $\mathrm{D}$

1.34E-17

sulfotransferase family, cytosolic, 1C, member 4

8.47E-17

Histamine-specific:

$\begin{array}{llc}\text { SERPINB2 } & \text { serpin peptidase inhibitor } & 4.43 \mathrm{E}-130 \\ \text { FST } & \text { follistatin } & 1.24 \mathrm{E}-106 \\ \text { AKAP12 } & \text { A kinase (PRKA) anchor protein 12 } & 7.51 \mathrm{E}-85 \\ \text { RELN } & \text { reelin } & 2.02 \mathrm{E}-72 \\ \text { PLAU } & \text { plasminogen activator, urokinase } & 4.08 \mathrm{E}-59 \\ \text { PLAT } & \text { plasminogen activator, tissue } & 6.55 \mathrm{E}-54 \\ \text { SEMA3A } & \text { semaphorin3A } & 4.40 \mathrm{E}-50 \\ \text { MALL } & \text { mal, T-cell differentiation protein-like } & 1.11 \mathrm{E}-46 \\ \text { PEG10 } & \text { paternally expressed 10 } & 1.36 \mathrm{E}-42 \\ \text { MYADM } & \text { myeloid-associated differentiation marker } & 3.23 \mathrm{E}-42\end{array}$

*Upregulated transcription factors and transcription enhancer factors.

Table 3. GO analysis of commonly regulated genes in HUVEC cells after 7h of VEGF and histamine exposure.

\begin{tabular}{|c|c|c|}
\hline GO & Term & Genes \\
\hline GO:0045595 & regulation of cell differentiation & PTHLH,DLL1,FITM2,AQP1,MAFB,KITLG,RCAN1, \\
\hline GO:0050793 & and developmental process & $\begin{array}{l}\text { SMOC1,HGF,GDPD5,SEMA6A,ABCA1,HDAC9, } \\
\text { GDF7 }\end{array}$ \\
\hline GO:0048731 & \multirow[t]{3}{*}{ organ, system and tissue development } & CLDN5,PTHLH,NR4A1,AQP1,MYO7A,MAFB, \\
\hline GO:0009888 & & KALRN,TNFSF10,SMOC1,RCAN1,GDPD5, \\
\hline GO:0048513 & & $\begin{array}{l}\text { SEMA6A,DOK5,HDAC9,CYP1A1,DLL1,KITLG, } \\
\text { HGF, SLC12A2, STC1,NLGN4Y,GDF7 }\end{array}$ \\
\hline \multirow[t]{4}{*}{ GO:0007154 } & \multirow[t]{4}{*}{ cell communication } & PTHLH,CCL20,NR4A1,PMCH,A2M,KALRN,TNFSF \\
\hline & & 10,DNAJB11,SMOC1,RCAN1,RASD1,SEMA6A, \\
\hline & & DOK5,HDAC9,DLL1,BCL2A1,KITLG,ARHGAP28, \\
\hline & & OPRL1,HGF,ANXA9,SLC12A2,ABCA1,STC1,GDF7 \\
\hline GO:0071320 & cellular response to cAMP & STC1,AQP1 \\
\hline GO:0002673 & regulation of acute inflammatory response & ALOX5AP,A2M \\
\hline GO:0007186 & $\begin{array}{l}\text { G-protein coupled receptor signaling } \\
\text { pathway }\end{array}$ & RASD1,OPRL1,PTHLH,SLC12A2,PMCH,ABCA1 \\
\hline
\end{tabular}


Table 4. Gene Regulation of Major Signaling Pathways by VEGF and Histamine in HUVEC cells after 7h of exposure.

\begin{tabular}{|c|c|c|}
\hline IDterm & Term & Genes \\
\hline \multirow[t]{4}{*}{ hsa04060 } & Cytokine-cytokine receptor interaction: & \\
\hline & VEGF-A & KITLG,TNFSF10,HGF,CCL20 \\
\hline & Histamine & CCL7,BMP2,TNFSF13,CXCL11,CCL20*,IL8 \\
\hline & & TNFSF10*, KITLG*,HGF*,GDF5 \\
\hline \multirow[t]{3}{*}{ hsa04151 } & PI3K-Akt signaling pathway: & \\
\hline & VEGF-A & PDGFD,KITLG,HGF,BCL2L11,NR4A1 \\
\hline & Histamine & KITLG*,HGF* ,RELN,ITGB8,NR4A1* \\
\hline \multirow[t]{2}{*}{ hsa04610 } & Complement and coagulation cascades: & \\
\hline & Histamine & PLAU,PLAT,SERPIND1,A2M* \\
\hline \multirow[t]{2}{*}{ hsa04068 } & FoxO signaling pathway: & \\
\hline & VEGF-A & TNFSF10*,FBXO32*,BCL2L11 \\
\hline hsa04015, & Rap1 and Ras signaling pathways: & \\
\hline \multirow[t]{2}{*}{ hsa04014 } & VEGF-A & PDGFD,KITLG,HGF \\
\hline & Histamine & KITLG* ${ }^{*} \mathrm{HGF}^{*}, \mathrm{MAP} 2 \mathrm{~K} 6$ \\
\hline \multirow[t]{2}{*}{ hsa04350 } & TGF-beta signaling pathway: & \\
\hline & Histamine & BMP2,FST,GDF5,GDF7* \\
\hline \multirow[t]{2}{*}{ hsa04064 } & NF-kappa B signaling pathway: & \\
\hline & Histamine & BIRC3,PLAU,BCL2A1*,IL8 \\
\hline \multirow[t]{2}{*}{ hsa04668 } & TNF signaling pathway: & \\
\hline & Histamine & BIRC3,CCL20*,RPS6KA5,MAP2K6 \\
\hline \multirow[t]{2}{*}{ hsa04390 } & Hippo signaling pathway: & \\
\hline & Histamine & BMP2,GDF5,TEAD2,GDF7* \\
\hline
\end{tabular}




\section{DISCUSSION}

Physiological effects of VEGFs and histamine on the vasculature are well established but postreceptor signaling pathways and gene regulation affecting angiogenic signals are not fully understood. Using RNA-seq and functional assays, we compared gene regulation by VEGF and histamine in human primary ECs and in the intact vasculature of C57/BI6 mice after systemic delivery. Altogether, 76 commonly regulated genes were found between histamine and VEGF stimulated ECs by RNA-seq, representing $53 \%$ of all VEGF-regulated transcripts and $\sim 26 \%$ of all histamine-regulated transcripts. Several of the genes encoded proteins important for cell junction stability, cytoskeleton organization and cell-ECM adhesion thereby affecting EC integrity and proliferation. Particularly, VEGF induced changes in the immunoglobulin superfamily cell adhesion receptors and genes involved in the stabilizing/regulating TJs and AJs, whereas histamine regulated mainly transcripts linked to TJ function. Both histamine and VEGF reduced expression of the major TJ protein claudin5 that has previously been connected to pathological angiogenesis, increased macromolecule influx (18) and blood-brain barrier breakdown (19). Although changes in VEcadherin and F-actin organization at short-time points after stimulation were found, no change in gene expression level of VE-cadherin was detected with either histamine or VEGF in our study. This indicates that claudin- 5 may be more regulated at the transcriptional level, whereas VE-cadherin is mainly regulated at the post-translational level (20). In addition to claudin-5, histamine upregulated several cell adhesion molecules that are a known hallmark of inflammation and H1R-mediated signaling. Although Schweighofer et al. (2009) suggested earlier a similar expression pattern of inflammatory genes by VEGF and IL-1 (21), these changes were not detected in our study.

Decreased cell-cell adhesion has been reported to promote angiogenesis and is likely regulated by multiple factors at the gene expression level. Previously, histamine has been suggested to promote vessel growth in tumors, as well as to induce EC proliferation and angiogenesis by upregulating VEGF expression or by inducing expression of the proangiogenic transcription factor NR4A1 $(6,22)$. In our study, no upregulation of VEGFs or VEGFRs was detected after histamine stimulation. Additionally, inhibition experiments by VEGFR2 siRNA did not reduce histamine-mediated regulation of specific genes (e.g. RCAN1, CLDN5). Therefore, our results are in line with the previous finding of Qin et al. (6) that histamine is able to induce VEGFR2 independent angiogenic signaling. Moreover, we also show that the cellular mechanisms required to induce angiogenic processes by histamine are more complex than earlier reported direct regulation via NR4A1 and instead involves changes in the expression levels of multiple genes, including growth factors, cytokines, transcription factors and calcium dependent cell signaling events. Altogether, 14 commonly regulated genes were directly involved in cell proliferation by histamine and VEGF. A common mediator of EC proliferation was shown to be HGF, which has previously been linked to induce EC proliferation via PI3K-Akt pathway (23), and to be upregulated after ischemic injury (24) and in cancer (23). HGF, however, had no effect on claudin-5 expression and subsequent TJ formation. To identify the transcription factors required for cell adhesion/proliferation by histamine and VEGF, functional studies were further performed based on RNA-seq data. Particularly the role of transcription factors MYCN, NR4A1, SNAI2 and RCAN1 was further investigated due to their high upregulation by either histamine and/or VEGF. MYCN and SNAI2 were exclusively regulated by VEGF, whereas NR4A1 and 140 
RCAN1 were regulated by both factors. MYCN expression has been previously linked to integrin-mediated cell adhesion and PI3K-mediated VEGF regulation in neuroblastoma (25). SNAI2 has been shown to repress claudin-1 expression in keratinocytes (26) and to be upregulated in pathological angiogenesis. RCAN1 has been connected to VEGF-signaling and regulation of angiogenesis via NFAT-signaling (27). Prior to this study, histamine signaling has not been connected to these genes. Furthermore, we demonstrated that NR4A1, MYCN and SNAI2 induce downregulation of claudin-5, and are required for EC proliferation. Since both NR4A1 and RCAN1 have been shown to be upregulated after elevated intracellular calcium levels $(28,29)$ and changes in gene expression of calcium transporters were detected with both histamine and VEGF, this together with HGF induced signaling may explain the observed similar gene expression patterns found between the factors. Although the role of calcium in mediating angiogenesis needs further clarification, calcium overload seems to lead to upregulation of multiple TFs (30) that promote angiogenic processes in ECs by histamine and VEGF.

As VEGFs have been used to induce arteriogenesis and angiogenesis in the clinic to treat coronary and peripheral vascular diseases, understanding their potential adverse effects is of great importance for future clinical use. Our group previously demonstrated that VEGF therapy in preclinical animal models successfully induced blood vessel growth, increased skeletal muscle perfusion but simultaneously caused edema (31). So far the molecular mechanisms regulating cell-cell adhesion and EC permeability have been poorly understood, although numerous genes have been linked with vascular permeability (32). We show here that systemic delivery of histamine and VEGF-F causes vascular hyperpermeability of $200 \mathrm{~nm}$ sized particles $(<10 \mathrm{~min})$ in the intact vasculature of mice, whereas VEGF-A $A_{165}$ induces a slower hyper-permeability response (<30min). Previously, the majority of vascular permeability studies have been performed locally in skin by using the modified Miles Assay. However, the Miles assay although effective, has been shown to induce inflammation at site or in individually perfused vessels (33). Based on these methods, VEGF$F$ has been suggested as a more potent vasodilator than VEGF-A $A_{165}$ (34), whereas VEGF- $A_{121}$ and VEGF-A $A_{165}$ induce similar local responses (12). Our data, however, show that VEGFinduced vascular hyper-permeability after systemic delivery i) varies among different vascular beds and ii) is time-dependent. Both VEGF- $\mathrm{A}_{165}$ and VEGF-F cause similar responses over time. Time-dependent variation of VEGFs is likely explained by VEGF-A $A_{165}$ binding to VEGFR1/VEGFR2, whereas VEGF-F only binds to VEGFR2. To our knowledge this is the first comprehensive study evaluating VEGF-induced effects on vascular permeability in intact vasculature of mice in various vascular beds. Additionally, in our EC permeability model, VEGF-A $A_{121}$ was not able to induce EC permeability or induce changes in VE-cadherin localization or F-actin organization. Additional differences were detected in the expression levels of calcium-induced genes (e.g. ATP2B1, SLC12A2, STC1) by VEGF-A 121 and other VEGFR2-binding VEGFs. The obtained knowledge of VEGF-induced vascular hyperpermeability responses are valuable in the future for safety evaluation of these proteins for pro-angiogenic therapy.

Previously, it has been suggested that endothelial function requires regulation - not only at transient phosphorylation level - but also at transcriptional level (35). In this study, we identified novel common molecular players influencing EC activation, proliferation and adhesion by VEGFs and histamine. RNA-seq was used to identify gene regulation behind angiogenic signals. Our results on the regulation of vascular endothelium improve general 
understanding of pathological angiogenesis and may provide novel treatment targets, such as RCAN1, MYCN and SNAI2 for treatment of vascular pathologies. Regulation of e.g. MYCN signaling by CDK7 inhibitors (36) or NR4A1 signaling by calcineurin inhibitors (37) may also be beneficial in pro-angiogenic therapy to reduce edema and EC proliferation. 


\section{ACKNOWLEDGMENTS}

This study was supported by Academy of Finland (project No 250614), CoE of Cardiovascular and Metabolic Disease, ERC advanced grant (AdG09-250050) and CARIM PhD and VENI fellowships of the Netherlands Organization of Scientific Research (016.116.017). Personnel of the Kuopio University Hospital Maternity Ward are thanked for providing the umbilical cords. 


\section{REFERENCES}

1. Kumar P, Shen Q, Pivetti CD, Lee ES, Wu MH, Yuan SY. Molecular mechanisms of endothelial hyperpermeability: implications in inflammation. Expert Rev Mol Med. 2009 Jun;11:e19.

2. Olsson A-K, Dimberg A, Kreuger J, Claesson-Welsh L. VEGF receptor signalling - in control of vascular function. Nat Rev Mol Cell Biol. 2006 May;7(5):359-71.

3. Asai K, Kanazawa H, Kamoi H, Shiraishi S, Hirata K, Yoshikawa J. Increased levels of vascular endothelial growth factor in induced sputum in asthmatic patients. Clin Exp Allergy. 2003 May;33(5):595-9.

4. Brouillard P, Vikkula M. Vascular malformations: localized defects in vascular morphogenesis. Clin Genet. 2003 May;63(5):340-51.

5. Abdel-Majid RM, Marshall JS. Prostaglandin E2 induces degranulation-independent production of vascular endothelial growth factor by human mast cells. J Immunol. 2004 Jan 15;172(2):1227-36.

6. Qin L, Zhao D, Xu J, Ren X, Terwilliger EF, Parangi S, et al. The vascular permeabilizing factors histamine and serotonin induce angiogenesis through TR3/Nur77 and subsequently truncate it through thrombospondin-1. Blood. 2013 Mar 14;121(11):2154-64.

7. Norrby K. Evidence of a dual role of endogenous histamine in angiogenesis. Int J Exp Pathol. 1995 Apr;76(2):87-92.

8. Zauberman H, Michaelson IC, Bergmann F, Maurice DM. Stimulation of neovascularization of the cornea by biogenic amines. Exp Eye Res. 1969 Jan;8(1):77-83.

9. Ghosh AK, Hirasawa N, Ohtsu H, Watanabe T, Ohuchi K. Defective angiogenesis in the inflammatory granulation tissue in histidine decarboxylase-deficient mice but not in mast cell-deficient mice. J Exp Med. 2002 Apr 15;195(8):973-82.

10. Yang XD, Ai W, Asfaha S, Bhagat G, Friedman RA, Jin G, et al. Histamine deficiency promotes inflammation-associated carcinogenesis through reduced myeloid maturation and accumulation of CD11b+Ly6G+ immature myeloid cells. Nat Med. 2011 Jan;17(1):87-95.

11. Toivanen PI, Nieminen T, Viitanen L, Alitalo A, Roschier M, Jauhiainen S, et al. Novel vascular endothelial growth factor D variants with increased biological activity. J Biol Chem. 2009 Jun 5;284(23):16037-48

12. Nieminen T, Toivanen PI, Rintanen N, Heikura T, Jauhiainen S, Airenne KJ, et al. The impact of the receptor binding profiles of the vascular endothelial growth factors on their angiogenic features. Biochim Biophys Acta. 2014 Jan;1840(1):454-63.

13. Jaffe EA, Nachman RL, Becker CG, Minick CR. Culture of human endothelial cells derived from umbilica veins. Identification by morphologic and immunologic criteria. J Clin Invest. 1973 Nov;52(11):2745-56.

14. Abràmoff MD, Magalhães PJ, Ram SJ. Image processing with ImageJ. Biophotonics Int. 2004;11:36-42.

15. Wang D, Garcia-Bassets I, Benner C, Li W, Su X, Zhou Y, et al. Reprogramming transcription by distinct classes of enhancers functionally defined by eRNA. Nature. 2011 Jun 16;474(7351):390-4.

16. Heinz S, Benner C, Spann N, Bertolino E, Lin YC, Laslo P, et al. Simple combinations of lineagedetermining transcription factors prime cis-regulatory elements required for macrophage and $B$ cell identities. Mol Cell. 2010 May 28;38(4):576-89.

17. Robinson MD, McCarthy DJ, Smyth GK. edgeR: a Bioconductor package for differential expression 
analysis of digital gene expression data. Bioinformatics. 2010 Jan 1;26(1):139-40.

18. Wessel F, Winderlich M, Holm M, Frye M, Rivera-Galdos R, Vockel M, et al. Leukocyte extravasation and vascular permeability are each controlled in vivo by different tyrosine residues of VE-cadherin. Nat Immunol. 2014 Mar;15(3):223-30.

19. Argaw AT, Gurfein BT, Zhang Y, Zameer A, John GR. VEGF-mediated disruption of endothelial CLN-5 promotes blood-brain barrier breakdown. Proc Natl Acad Sci U S A. 2009 Feb 10;106(6):1977-82.

20. Gavard J, Gutkind JS. VEGF controls endothelial-cell permeability by promoting the beta-arrestindependent endocytosis of VE-cadherin. Nat Cell Biol. 2006 Nov;8(11):1223-34.

21. Schweighofer B, Testori J, Sturtzel C, Sattler S, Mayer H, Wagner O, et al. The VEGF-induced transcriptional response comprises gene clusters at the crossroad of angiogenesis and inflammation. Thromb Haemost. 2009 Sep;102(3):544-54.

22. Zeng H, Qin L, Zhao D, Tan X, Manseau EJ, Van Hoang M, et al. Orphan nuclear receptor TR3/Nur77 regulates VEGF-A-induced angiogenesis through its transcriptional activity. J Exp Med. 2006 Mar 20;203(3):719-29.

23. Naran S, Zhang X, Hughes SJ. Inhibition of HGF/MET as therapy for malignancy. Expert Opin Ther Targets. 2009 May;13(5):569-81.

24. Jennische E, Ekberg S, Matejka GL. Expression of hepatocyte growth factor in growing and regenerating rat skeletal muscle. Am J Physiol. 1993 Jul;265(1 Pt 1):C122-8.

25. Kang J, Rychahou PG, Ishola TA, Mourot JM, Evers BM, Chung DH. N-myc is a novel regulator of PI3Kmediated VEGF expression in neuroblastoma. Oncogene. 2008 Jun 26;27(28):3999-4007.

26. Martínez-Estrada OM, Cullerés A, Soriano FX, Peinado H, Bolós V, Martínez FO, et al. The transcription factors Slug and Snail act as repressors of Claudin-1 expression in epithelial cells. Biochem J. 2006 Mar 1;394(Pt 2):449-57.

27. Baggott RR, Alfranca A, López-Maderuelo D, Mohamed TMA, Escolano A, Oller J, et al. Plasma membrane calcium ATPase isoform 4 inhibits vascular endothelial growth factor-mediated angiogenesis through interaction with calcineurin. Arterioscler Thromb Vasc Biol. 2014 Oct;34(10):2310-20.

28. Pinato G, Pegoraro S, Visentini M, Ruaro ME, Torre V. Elevation of somatic Ca2+ upregulates genes Nr4a1 and Egr2, but not Bdnf and Arc. Neuroreport. 2009 Jun 17;20(9):869-74.

29. Sobrado M, Ramirez BG, Neria F, Lizasoain I, Arbones ML, Minami T, et al. Regulator of calcineurin 1 (Rcan1) has a protective role in brain ischemia/reperfusion injury. J Neuroinflammation. 2012;9(1):48.

30. Mellström B, Savignac M, Gomez-Villafuertes R, Naranjo JR. Ca2+-operated transcriptional networks: molecular mechanisms and in vivo models. Physiol Rev. 2008 Apr;88(2):421-49.

31. Rissanen TT, Korpisalo P, Markkanen JE, Liimatainen T, Ordén M-R, Kholová I, et al. Blood flow remodels growing vasculature during vascular endothelial growth factor gene therapy and determines between capillary arterialization and sprouting angiogenesis. Circulation. 2005 Dec 20;112(25):393746.

32. Nagy JA, Benjamin L, Zeng H, Dvorak AM, Dvorak HF. Vascular permeability, vascular hyperpermeability and angiogenesis. Angiogenesis. 2008 Jun 22;11(2):109-19.

33. Fu BM, Shen S. Acute VEGF effect on solute permeability of mammalian microvessels in vivo. Microvasc Res. 2004 Jul;68(1):51-62. 
34. Matsunaga Y, Yamazaki Y, Suzuki H, Morita T. VEGF-A and VEGF-F evoke distinct changes in vascular ultrastructure. Biochem Biophys Res Commun. 2009 Feb 20;379(4):872-5.

35. Goddard LM, Iruela-Arispe ML. Cellular and molecular regulation of vascular permeability. Thromb Haemost. 2013 Mar;109(3):407-15.

36. Chipumuro E, Marco E, Christensen CL, Kwiatkowski N, Zhang T, Hatheway CM, et al. CDK7 inhibition suppresses super-enhancer-linked oncogenic transcription in MYCN-driven cancer. Cell. 2014 Nov 20;159(5):1126-39.

37. Wang X, Hayashi S, Umezaki M, Yamamoto T, Kageyama-Yahara N, Kondo T, et al. Shikonin, a constituent of Lithospermum erythrorhizon exhibits anti-allergic effects by suppressing orphan nuclear receptor Nr4a family gene expression as a new prototype of calcineurin inhibitors in mast cells. Chem Biol Interact. 2014 Oct 29;224C:117-27. 


\section{SUPPLEMENTAL DATA}
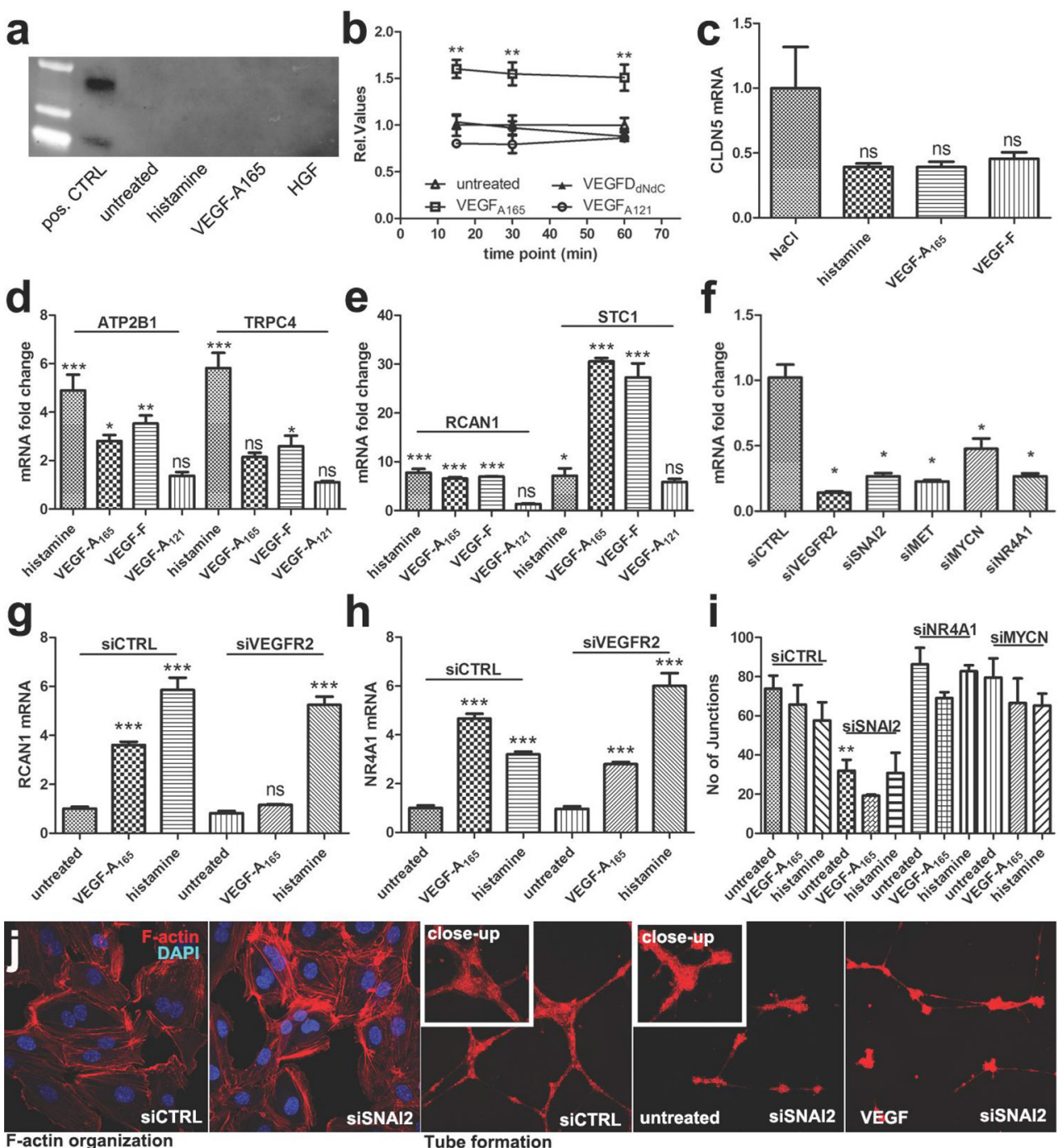

Supplemental figure 1. Regulation of Cell-Cell Adhesion Genes and Calcium Signaling Varies between VEGF Family Members

(A) No visible VEGF expression was detected in HUVEC cells after 7h stimulation with histamine, VEGF or HGF detected by WB. Monomer and dimer forms of VEGF are detected in the VEGF protein control (positive control). (B) Transwell assay was performed in HUVECs to study EC permeability induced by VEGF-A121, VEGF-A165 and VEGF-DdNdC. After the cells reached confluency, VEGFs were incubated together with Lucifer yellow that was used as an EC permeability marker. Nontreated cells were used as a control. Fluorescence was detected from the lower Transwell chamber at 15, 30 and $60 \mathrm{~min}$ time points by fluorometer. Relative values are shown together with mean \pm SEM. Significant EC permeability is detected with VEGF-A165. (C) TJ protein expression in lung. CLDN5 expression in lung tissue after $4 \mathrm{~h}$ of systemic delivery of VEGFs or histamine detected by qRT-PCR. (D-E) mRNA expression levels of genes involved in calcium signaling detected by qRT-PCR. Expression levels of plasma membrane calcium transporting ATPase 1 (ATP2B1; D), short transient receptor potential channel 4 (TRPC4; D), regulator of calcineurin 1 (RCAN1; E) and stanniocalcin-1 (STC1; E) after 7h stimulation with histamine or VEGFs in 
HUVECs are shown. (F) Silencing of target genes by siRNAs in HUVECs. Relative values are shown. (G-H) Role of VEGFR2 signaling in regulating RCAN1 and NR4A1expression. RCAN1 or NR4A1 mRNA expression after 7h stimulation with histamine or VEGFs in siVEGFR2 or siCTRL silenced HUVECs detected by qRT-PCR. I) Angiogenic sprouting was decreased in siSNAI2-treated cells. In vitro tube formation assay in HUVEC cells after $48 \mathrm{~h}$ siRNA treatment with siSNAI2, siNR4A1, siMYCN or siCTRL and after $7 \mathrm{~h}$ stimulation with VEGF or histamine. The amount of EC junctions is shown (4 images/treatment). (J) F-actin organization is changed in siSNAI2-treated HUVECs. Factin (red) was immunolabeled from confluent cell cultures and after in vitro tube formation assay (48h). VEGF stimulation was performed for $7 \mathrm{~h}$. In all data, mean+SEM presented. P-values: $<0.05^{*},<0.01^{* *},<0.001^{* * *}$. 


\begin{tabular}{|c|c|c|c|c|c|c|}
\hline \multicolumn{3}{|c|}{ SUPPLEMENTARY TABLE 1. COMMONLY REGULATED GENES BY VEGF AND HISTAMINE. } & \multicolumn{2}{|l|}{ VEGF } & \multicolumn{2}{|c|}{ Histamine } \\
\hline Gene ID & Symbol & Description & $\log \mathrm{FC}$ & P-Value & $\log \mathrm{FC}$ & P-Value \\
\hline NM_002674 & PMCH & pro-melanin-concentrating hormone & 2,69 & $2,27 \mathrm{E}-07$ & 4,41 & $8,11 \mathrm{E}-34$ \\
\hline NM_001130046 & CCL20 & chemokine (C-C motif) ligand 20 & 1,81 & 2,33E-03 & 3,77 & 6,19E-19 \\
\hline NM_030915 & LBH & limb bud and heart development & 1,40 & $5,01 \mathrm{E}-15$ & 2,58 & $4,26 E-59$ \\
\hline NM_004049 & BCL2A1 & $\mathrm{BCL} 2$-related protein $\mathrm{A} 1$ & 1,11 & $4,90 \mathrm{E}-02$ & 2,14 & $1,07 \mathrm{E}-05$ \\
\hline NM_017846 & TRNAU1AP & tRNA selenocysteine 1 associated protein 1 & 1,87 & 3,10E-04 & 2,13 & $1,01 \mathrm{E}-05$ \\
\hline NM_203418 & RCAN1 & regulator of calcineurin 1 & 1,23 & $4,24 E-29$ & 2,13 & $8,76 E-81$ \\
\hline NM_003155 & STC1 & stanniocalcin 1 & 5,03 & $3,09 \mathrm{E}-133$ & 2,11 & $2,16 \mathrm{E}-11$ \\
\hline NM_003994 & KITLG & KIT ligand & 1,21 & $1,33 \mathrm{E}-21$ & 1,80 & $3,79 E-30$ \\
\hline NM_003462 & DNALII & dynein, axonemal, light intermediate chain 1 & 1,70 & $2,09 \mathrm{E}-15$ & 1,67 & $7,98 \mathrm{E}-16$ \\
\hline NM_002135 & NR4A1 & nuclear receptor subfamily 4 , group A, member 1 & 2,34 & $3,38 \mathrm{E}-21$ & 1,66 & $1,26 \mathrm{E}-09$ \\
\hline NM_001204148 & HDAC9 & histone deacetylase 9 & 1,14 & $1,90 \mathrm{E}-05$ & 1,59 & $2,79 \mathrm{E}-10$ \\
\hline NM_018431 & DOK5 & docking protein 5 & 0,71 & 3,90E-03 & 1,57 & $9,74 \mathrm{E}-13$ \\
\hline NM_145728 & SYNM & synemin, intermediate filament protein & 1,05 & $2,12 \mathrm{E}-15$ & 1,47 & $6,18 \mathrm{E}-25$ \\
\hline NM_198965 & PTHLH & parathyroid hormone-like hormone & 1,15 & $6,60 \mathrm{E}-03$ & 1,45 & $3,94 \mathrm{E}-04$ \\
\hline NM_001034852 & SMOC1 & SPARC related modular calcium binding 1 & 1,07 & $5,00 \mathrm{E}-03$ & 1,42 & $9,08 \mathrm{E}-05$ \\
\hline NM_001271004 & TFPI2 & tissue factor pathway inhibitor 2 & 0,81 & 3,13E-18 & 1,41 & $1,52 \mathrm{E}-42$ \\
\hline NM_001086 & AADAC & arylacetamide deacetylase & 1,02 & $2,84 \mathrm{E}-05$ & 1,25 & $1,55 \mathrm{E}-07$ \\
\hline NM_001145349 & IPP & intracisternal A particle-promoted polypeptide & 1,10 & $5,81 \mathrm{E}-05$ & 1,21 & $1,35 \mathrm{E}-05$ \\
\hline NM_000601 & HGF & hepatocyte growth factor (hepapoietin A; scatter factor) & 0,94 & $3,95 \mathrm{E}-03$ & 1,20 & $1,34 \mathrm{E}-04$ \\
\hline NM_001008739 & C6orf226 & chromosome 6 open reading frame 226 & 1,29 & $1,02 \mathrm{E}-03$ & 1,16 & $4,35 \mathrm{E}-03$ \\
\hline NM_001001323 & ATP2B1 & ATPase, Ca++ transporting, plasma membrane 1 & 0,80 & $7,62 \mathrm{E}-11$ & 1,12 & $1,14 \mathrm{E}-21$ \\
\hline NM_001242463 & FBX032 & F-box protein 32 & 0,73 & 4,97E-06 & 1,12 & $5,92 \mathrm{E}-15$ \\
\hline NM_000014 & A2M & alpha-2-macroglobulin & 1,98 & $4,44 \mathrm{E}-16$ & 1,11 & 1,15E-04 \\
\hline NM_001629 & ALOX5AP & arachidonate 5-lipoxygenase-activating protein & 1,05 & $4,38 \mathrm{E}-02$ & 1,06 & $4,24 \mathrm{E}-02$ \\
\hline NM_001007595 & C2CD4B & C2 calcium-dependent domain containing 4B & 1,12 & $8,32 \mathrm{E}-04$ & 1,04 & 3,43E-03 \\
\hline NM_001256461 & SLC12A2 & solute carrier family 12 , member 2 & 0,74 & $9,44 \mathrm{E}-17$ & 1,04 & $1,10 \mathrm{E}-26$ \\
\hline NR_034078 & LOC643733 & caspase 4 , apoptosis-related cysteine peptidase pseudogene & 0,85 & $5,29 E-05$ & 1,01 & 4,86E-06 \\
\hline NM_032854 & CORO6 & coronin 6 & 0,79 & $1,20 \mathrm{E}-02$ & 1,00 & $1,96 \mathrm{E}-03$ \\
\hline NM_148909 & OSBPL9 & oxysterol binding protein-like 9 & 0,80 & $4,66 \mathrm{E}-02$ & 0,97 & $8,70 \mathrm{E}-03$ \\
\hline NM_016306 & DNAJB11 & DnaJ (Hsp40) homolog, subfamily B, member 11 & 0,73 & $1,74 \mathrm{E}-15$ & 0,96 & $1,40 \mathrm{E}-24$ \\
\hline NM_138464 & C5orf55 & chromosome 5 open reading frame 55 & 1,06 & $5,47 \mathrm{E}-03$ & 0,94 & $1,74 \mathrm{E}-02$ \\
\hline NR_002775 & RPLPOP2 & ribosomal protein, large, PO pseudogene 2 & 0,74 & $3,31 \mathrm{E}-07$ & 0,93 & 4,05E-09 \\
\hline NM_021237 & SELK & selenoprotein $\mathrm{K}$ & 0,75 & $3,89 \mathrm{E}-09$ & 0,93 & $3,21 \mathrm{E}-15$ \\
\hline NM_005460 & SNCAIP & synuclein, alpha interacting protein & 0,91 & $3,41 \mathrm{E}-06$ & 0,92 & $2,90 \mathrm{E}-06$ \\
\hline NR_037901 & ARHGEF26-AS1 & ARHGEF26 antisense RNA 1 & 0,84 & 2,99E-02 & 0,86 & $2,61 \mathrm{E}-02$ \\
\hline NR_024124 & ATP1A10S & ATP1A1 opposite strand & 1,02 & $1,84 \mathrm{E}-17$ & 0,86 & $6,74 \mathrm{E}-12$ \\
\hline $\begin{array}{l}\text { NM_198148 } \\
\text { NM_016084 }\end{array}$ & $\begin{array}{l}\text { CPXM2 } \\
\text { RASD1 }\end{array}$ & $\begin{array}{l}\text { carboxypeptidase } X \text { (M14 family), member } 2 \\
\text { RAS, dexamethasone-induced } 1\end{array}$ & $\begin{array}{l}1,57 \\
1,19\end{array}$ & $\begin{array}{l}4,56 \mathrm{E}-15 \\
2,96 \mathrm{E}-09\end{array}$ & $\begin{array}{l}0,82 \\
0,81\end{array}$ & $\begin{array}{l}2,38 \mathrm{E}-04 \\
7,15 \mathrm{E}-04\end{array}$ \\
\hline NM_199242 & UNC13D & unc-13 homolog D (C. elegans) & $-0,95$ & $6,64 \mathrm{E}-05$ & $-0,66$ & $3,77 \mathrm{E}-03$ \\
\hline NM_001080472 & FITM2 & fat storage-inducing transmembrane protein 2 & $-0,94$ & $3,42 \mathrm{E}-03$ & $-0,71$ & $3,15 \mathrm{E}-02$ \\
\hline NM_198540 & B3GNT8 & UDP-GICNAc: betaGal beta-1,3-N-acetylglucosaminyltransferase 8 & $-1,00$ & $1,59 \mathrm{E}-04$ & $-0,73$ & $6,68 \mathrm{E}-03$ \\
\hline NM_001080509 & TSPAN11 & tetraspanin 11 & $-0,87$ & $6,54 \mathrm{E}-09$ & $-0,74$ & $7,56 \mathrm{E}-07$ \\
\hline NM_001164238 & NLGN4Y & neuroligin 4, Y-linked & $-1,26$ & $1,75 \mathrm{E}-03$ & $-0,79$ & $3,59 E-02$ \\
\hline NR_037844 & LOC100133445 & uncharacterized LOC100133445 & $-1,00$ & $1,58 \mathrm{E}-03$ & $-0,83$ & $9,84 \mathrm{E}-03$ \\
\hline NM_001242835 & NDRG4 & NDRG family member 4 & $-0,72$ & $1,72 \mathrm{E}-12$ & $-0,86$ & $4,06 \mathrm{E}-17$ \\
\hline NM_014746 & RNF144A & ring finger protein $144 \mathrm{~A}$ & $-1,30$ & $1,09 \mathrm{E}-08$ & $-0,88$ & $3,21 \mathrm{E}-05$ \\
\hline NM_032385 & FAXDC2 & fatty acid hydroxylase domain containing 2 & $-0,81$ & 2,13E-04 & $-0,88$ & $1,60 \mathrm{E}-04$ \\
\hline NM_005461 & MAFB & v-maf musculoaponeurotic fibrosarcoma oncogene homolog B (avian) & $-0,72$ & $5,84 \mathrm{E}-04$ & $-0,90$ & $2,86 \mathrm{E}-05$ \\
\hline NM_005618 & DLL1 & delta-like 1 (Drosophila) & $-0,85$ & 1,53E-03 & $-0,90$ & $1,15 \mathrm{E}-03$ \\
\hline NM_015016 & MAST3 & microtubule associated serine/threonine kinase 3 & $-0,74$ & $2,74 \mathrm{E}-03$ & $-0,91$ & $6,04 \mathrm{E}-04$ \\
\hline NM_020796 & SEMA6A & semaphorin 6A & $-1,12$ & $3,29 \mathrm{E}-05$ & $-0,93$ & $3,18 \mathrm{E}-04$ \\
\hline NM_001270765 & CHST15 & carbohydrate ( $\mathrm{N}$-acetylgalactosamine 4-sulfate 6-0) sulfotransferase 15 & $-0,83$ & $6,28 \mathrm{E}-04$ & $-0,93$ & 7,96E-05 \\
\hline NM_020939 & CPNE5 & copine V & $-0,90$ & $1,86 \mathrm{E}-05$ & $-0,94$ & $8,07 \mathrm{E}-07$ \\
\hline NM_001004470 & ST8SIA6 & ST8 alpha-N-acetyl-neuraminide alpha-2,8-sialyltransferase 6 & $-0,78$ & $3,05 E-02$ & $-0,95$ & $1,15 \mathrm{E}-02$ \\
\hline NM_003277 & CLDN5 & claudin 5 & $-1,08$ & $2,54 \mathrm{E}-23$ & $-0,96$ & $6,56 \mathrm{E}-17$ \\
\hline NM_002516 & NOVA2 & neuro-oncological ventral antigen 2 & $-0,78$ & $8,02 \mathrm{E}-06$ & $-0,96$ & $9,62 \mathrm{E}-08$ \\
\hline NM_001256067 & NOXA1 & NADPH oxidase activator 1 & $-1,54$ & $8,80 \mathrm{E}-03$ & $-0,97$ & $4,75 E-02$ \\
\hline NM_138445 & GPR146 & G protein-coupled receptor 146 & $-0,92$ & $3,97 \mathrm{E}-07$ & $-0,99$ & $1,44 \mathrm{E}-07$ \\
\hline NM_001190942 & TNFSF10 & tumor necrosis factor (ligand) superfamily, member 10 & $-1,00$ & $2,46 \mathrm{E}-13$ & $-1,05$ & $1,52 \mathrm{E}-12$ \\
\hline NM_198988 & LENG9 & leukocyte receptor cluster (LRC) member 9 & $-0,76$ & $1,52 \mathrm{E}-02$ & $-1,05$ & $2,06 \mathrm{E}-03$ \\
\hline NM_020716 & GRAMD1B & GRAM domain containing $1 \mathrm{~B}$ & $-0,93$ & $1,25 \mathrm{E}-02$ & $-1,10$ & $4,33 \mathrm{E}-03$ \\
\hline NM_001113567 & FAM211A & family with sequence similarity 211 , member $A$ & $-0,70$ & $5,22 \mathrm{E}-04$ & $-1,14$ & $1,78 \mathrm{E}-07$ \\
\hline NM_001024660 & KALRN & kalirin, RhoGEF kinase & $-0,71$ & $5,60 \mathrm{E}-05$ & $-1,17$ & $1,48 \mathrm{E}-09$ \\
\hline NM_030792 & GDPD5 & glycerophosphodiester phosphodiesterase domain containing 5 & $-1,07$ & $2,07 \mathrm{E}-11$ & $-1,17$ & $5,43 E-14$ \\
\hline NM_198098 & AQP1 & aquaporin 1 (Colton blood group) & $-2,19$ & $1,18 \mathrm{E}-34$ & $-1,25$ & $1,86 \mathrm{E}-13$ \\
\hline NM_001127180 & MYOTA & myosin VIIA & $-1,17$ & $1,87 \mathrm{E}-05$ & $-1,25$ & $1,92 \mathrm{E}-06$ \\
\hline NM_182828 & GDF7 & growth differentiation factor 7 & $-0,74$ & $1,53 \mathrm{E}-03$ & $-1,27$ & $5,89 \mathrm{E}-07$ \\
\hline NM_001010000 & ARHGAP28 & Rho GTPase activating protein 28 & $-0,81$ & $1,68 \mathrm{E}-06$ & $-1,42$ & $4,80 \mathrm{E}-15$ \\
\hline NM_001200019 & OPRL1 & opiate receptor-like 1 & $-0,79$ & $1,04 \mathrm{E}-02$ & $-1,43$ & $4,06 \mathrm{E}-05$ \\
\hline NM_207336 & ZNF467 & zinc finger protein 467 & $-1,39$ & $2,23 E-08$ & $-1,47$ & $9,01 \mathrm{E}-09$ \\
\hline NM_005502 & ABCA1 & ATP-binding cassette, sub-family A ( $A B C 1$ ), member 1 & $-1,38$ & $7,01 \mathrm{E}-20$ & $-1,51$ & $1,34 \mathrm{E}-21$ \\
\hline NM_021063 & HIST1H2BD & histone cluster $1, \mathrm{H} 2 \mathrm{bd}$ & $-1,31$ & 2,75E-02 & $-1,53$ & $1,20 \mathrm{E}-02$ \\
\hline NM_003568 & ANXA9 & annexin A9 & $-1,49$ & 1,13E-02 & $-1,56$ & $3,81 \mathrm{E}-03$ \\
\hline $\begin{array}{l}\text { NM_000499 } \\
\text { NM_152852 }\end{array}$ & $\begin{array}{l}\text { CYP1A1 } \\
\text { MS4A6A }\end{array}$ & $\begin{array}{l}\text { cytochrome P450, family } 1 \text {, subfamily A, polypeptide } 1 \\
\text { membrane-spanning } 4 \text {-domains, subfamily A, member } 6 \text { A }\end{array}$ & $\begin{array}{l}-1,97 \\
-1,10\end{array}$ & $\begin{array}{l}8,90 \mathrm{E}-10 \\
3,64 \mathrm{E}-02\end{array}$ & $\begin{array}{l}-1,68 \\
-2,02\end{array}$ & $\begin{array}{l}1,57 \mathrm{E}-06 \\
5,84 \mathrm{E}-04\end{array}$ \\
\hline NM_012419 & RGS17 & regulator of G-protein signaling 17 & $-1,79$ & $2,83 \mathrm{E}-02$ & $-3,72$ & $1,54 \mathrm{E}-04$ \\
\hline
\end{tabular}




\section{Supplementary table 2 KEGG pathways}

A. Selected VEGF-stimulated pathways.
\begin{tabular}{|l|l|l|r|l|}
\hline TermID & Term & logP & Target Genes in Term & Gene Symbols \\
\hline hsa05202 & Transcriptional misregulation in cancer & $-3,51$ & 4 & HIST1H3C,MYCN,BCL2A1,MEF2C \\
\hline hsa05203 & Viral carcinogenesis & $-3,07$ & 4 & HLA-G,EGR3,HIST1H2BD,HDAC9 \\
\hline hsa04068 & FoxO signaling pathway & $-2,88$ & 3 & TNFSF10,FBXO32,BCL2L11 \\
\hline hsa04514 & Cell adhesion molecules (CAMs) & $-2,70$ & 3 & HLA-G,CLDN5,JAM2 \\
\hline hsa04151 & PI3K-Akt signaling pathway & $-2,58$ & 5 & PDGFD,KITLG,HGF,BCL2L11,NR4A1 \\
\hline hsa04060 & Cytokine-cytokine receptor interaction & $-2,34$ & 4 & KITLG,TNFSF10,HGF,CCL20 \\
\hline hsa04015 & Rap1 signaling pathway & $-1,80$ & 3 & PDGFD,KITLG,HGF \\
\hline hsa04014 & Ras signaling pathway & $-1,68$ & 3 & PDGFD,KITLG,HGF \\
\hline
\end{tabular}

B. Selected histamine-stimulated pathways.

\begin{tabular}{|c|c|c|c|c|}
\hline TermID & Term & $\log P$ & Target Genes in Term & Gene Symbols \\
\hline hsa04060 & Cytokine-cytokine receptor interaction & $-5,92$ & 10 & CCL7,BMP2,TNFSF13,CXCL11,CCL20,IL8,TNFSF10,KITLG,HGF,GDF5 \\
\hline hsa05202 & Transcriptional misregulation in cancer & $-4,63$ & 7 & BIRC3,PLAU,BCL2A1,NUPR1,PLAT,HIST1H3D,IL8 \\
\hline hsa04514 & Cell adhesion molecules (CAMs) & $-4,46$ & 6 & CLDN1,NRXN3,CLDN5,ITGB8,CLDN14,PVRL1 \\
\hline hsa04610 & Complement and coagulation cascades & $-4,31$ & 4 & PLAU,PLAT,SERPIND1,A2M \\
\hline hsa04350 & TGF-beta signaling pathway & $-3,82$ & 4 & BMP2,FST,GDF5,GDF7 \\
\hline hsa05323 & Rheumatoid arthritis & $-3,48$ & 4 & TNFSF13,CCL20,IL8,ATP6V0A4 \\
\hline hsa04064 & NF-kappa B signaling pathway & $-3,41$ & 4 & BIRC3,PLAU,BCL2A1,IL8 \\
\hline hsa04261 & Adrenergic signaling in cardiomyocytes & $-3,02$ & 5 & ADRB2,ACTC1,RPS6KA5,ATP2B1,CREM \\
\hline hsa04668 & TNF signaling pathway & $-2,82$ & 4 & BIRC3,CCL20,RPS6KA5,MAP2K6 \\
\hline hsa05410 & Hypertrophic cardiomyopathy (HCM) & $-2,31$ & 3 & ACTC1,ITGB8,DMD \\
\hline hsa04512 & ECM-receptor interaction & $-2,23$ & 3 & RELN,GP6,ITGB8 \\
\hline hsa05414 & Dilated cardiomyopathy & $-2,13$ & 3 & ACTC1,ITGB8,DMD \\
\hline hsa04390 & Hippo signaling pathway & $-1,91$ & 4 & BMP2,GDF5,TEAD2,GDF7 \\
\hline hsa04620 & Toll-like receptor signaling pathway & $-1,78$ & 3 & CXCL11,MAP2K6,IL8 \\
\hline hsa04141 & Protein processing in endoplasmic reticulum & $-1,69$ & 4 & DNAJB11,HYOU1,DERL3,HSPA5 \\
\hline hsa04670 & Leukocyte transendothelial migration & $-1,56$ & 3 & CLDN1,CLDN5,CLDN14 \\
\hline hsa04360 & Axon guidance & $-1,42$ & 3 & SEMA3A,SEMA6A,SLIT2 \\
\hline hsa04062 & Chemokine signaling pathway & $-1,41$ & 4 & CCL7,CXCL11,CCL20,IL8 \\
\hline hsa04530 & Tight junction & $-1,32$ & 3 & CLDN1,CLDN5,CLDN14 \\
\hline hsa04510 & Focal adhesion & $-1,21$ & 4 & HGF,RELN,BIRC3,ITGB8 \\
\hline hsa05200 & Pathways in cancer & $-0,81$ & 5 & BMP2,KITLG,HGF,BIRC3,IL8 \\
\hline hsa04151 & PI3K-Akt signaling pathway & $-0,71$ & 5 & KITLG,HGF,RELN,ITGB8,NR4A1 \\
\hline hsa04080 & Neuroactive ligand-receptor interaction & $-0,68$ & 4 & OPRL1,SSTR1,ADRB2,HTR2B \\
\hline
\end{tabular}

C. Selected commonly regulated pathways by VEGF and histamine.

\begin{tabular}{|l|l|l|l|l|}
\hline TermID & Term & logP & Target Genes in Term & Gene Symbols \\
\hline hsa04060 & Cytokine-cytokine receptor interaction & $-3,99$ & 4 & KITLG,TNFSF10,HGF,CCL20 \\
\hline hsa04151 & PI3K-Akt signaling pathway & $-1,90$ & 3 & KITLG,HGF,NR4A1 \\
\hline
\end{tabular}


Supplementary tabe 3 Genes for qRT-PCR

\begin{tabular}{|c|c|c|}
\hline Gene & & Assay ID \\
\hline hANO1 & Anoctamin 1 (human) & Hs00216121_m1 \\
\hline hATP2B1 & ATPase, $\mathrm{Ca}++$ transporting plasma membrane 1 (human) & Hs00155949_m1 \\
\hline hB2M & Beta-2 microglobulin (human) & Hs00187842_m1 \\
\hline hCLDN5 & claudin 5 (human) & Hs00533949_s1 \\
\hline hCLDN14 & claudin 14 (human) & Hs99999034_m1 \\
\hline hHGF & hepatocyte growth factor (human) & Hs00300159_m1 \\
\hline hIL8 & interleukin 8 (human) & Hs99999034_m1 \\
\hline hMET & hepatocyte growth factor receptor (human) & Hs00179845_m1 \\
\hline hMYCN & $\begin{array}{l}\text { V-Myc Avian Myelocytomatosis Viral Oncogene } \\
\text { Neuroblastoma Derived (human) }\end{array}$ & Hs00232074_m1 \\
\hline $\mathrm{mMYCN}$ & $\begin{array}{l}\text { V-Myc Avian Myelocytomatosis Viral Oncogene } \\
\text { Neuroblastoma Derived (mouse) }\end{array}$ & Mm00476449_m1 \\
\hline hNR4A1 & nuclear receptor subfamily 4, group A, member 1 (human) & Hs00374226_m1 \\
\hline mNR4A1 & nuclear receptor subfamily 4, group A, member 1 (mouse) & Mm01300401_m1 \\
\hline mPPIA & peptidylprolyl Isomerase A (mouse) & Mm03302254_g1 \\
\hline hRCAN1 & regulator of calcineurin 1 (human) & Hs01120954_m1 \\
\hline hSLC12A2 & solute carrier family 12 member 2 (human) & Hs00169032_m1 \\
\hline hSTC1 & stanniocalcin 1 (human) & Hs01120954_m1 \\
\hline hTRPC4 & $\begin{array}{l}\text { transient receptor potential channel, subfamily } C \text {, } \\
\text { member } 4 \text { (human) }\end{array}$ & Hs01077392_m1 \\
\hline hVEGF & vascular endothelial growth factor (human) & Hs00900055_m1 \\
\hline hVEGFR2 & vascular endothelial growth factor receptor 2 (human) & Hs00911700_m1 \\
\hline
\end{tabular}


Chapter 6 


\section{Chapter 7}

\section{General discussion}

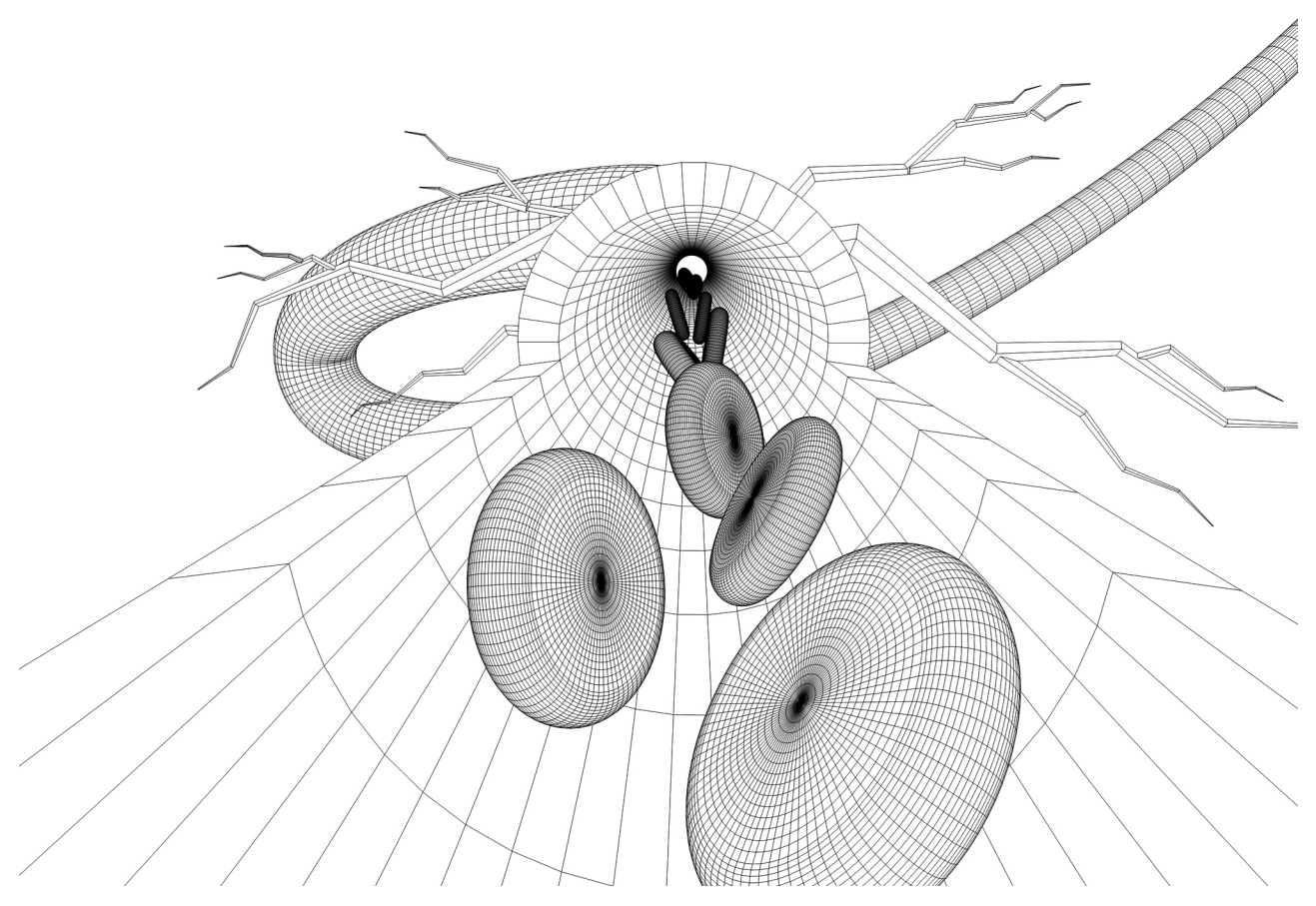




\section{Main findings of the thesis}

The overall aim of this thesis was to explore a possible causal role for plaque hypoxia and angiogenesis in experimental murine atherosclerosis, as both have been identified as risk factors for plaque rupture in humans.

Main findings of the presented work which are discussed in this chapter:

I. Hyperoxic therapy alleviates progression of experimental atherosclerosis (chapter 2) but the translation of our findings is complex.

II. Aggravation of the molecular hypoxia response stabilizes experimental atherosclerosis (chapter $\mathbf{3}$ ), but probable side effects require careful evaluation before translation.

III. Mouse models are not suitable to investigate intra-plaque angiogenesis in atherosclerosis (chapter $\mathbf{3}$, chapter $\mathbf{4}$, chapter $\mathbf{5}$ ).

IV. VEGF and histamine regulate vascular permeability via commonly and independently regulated pathways which might help to prevent edema formation in pro-angiogenic gene therapy (chapter 6).

\section{Hypoxia and atherosclerosis - translatability of own findings}

In chapter $\mathbf{2}$ we tested the hypothesis that hyperoxic treatment alleviates plaque hypoxia and atherosclerosis burden investigated the underlying molecular mechanism as plaque hypoxia is present in human atherosclerosis (1). A logical therapeutic intervention derived from the findings reported in chapter $\mathbf{2}$ is restoration of plaque oxygen levels in humans. In chapter $\mathbf{2}$ oxygen levels were restored in LDLr KO mice that had been on a Western type diet for 4 weeks, showing hypoxic fatty streaks. Oxygen levels were restored by daily inhalation of carbogen gas (95\% oxygen) over 4 weeks with continuation of Western type diet feeding. Plaque hypoxia was recognized already in 1944 by Hueper (2) and consequently oxygen therapy has been applied to treat experimental atherosclerosis (3-6). In comparison to Altschul et al. (3), Kjeldsen et al. (4) and Vesselinovitch et al. (6), we did not observe a reduction in plaque size, but rather a reduction in necrotic core percentage (chapter 2). A reduced necrotic core implies increased plaque stability. A major difference between chapter 2 and previous research is the use of mice instead of rabbits. The presence of more foam cells in rabbit lesions my, explain the different outcome (7). Other differences explaining the diverging results may be the study design; for instance Altschul et al. started the oxygen treatment together with the atherogenic diet, whereas Vesselinovitch et al. started the oxygen treatment after diet feeding, when atherosclerotic plaques had already formed. The study design of both studies seems irrelevant for human atherosclerosis as patients are not treated at disease initiation and disease progression likely continues after onset of the oxygen treatment. The in chapter $\mathbf{2}$ chosen experimental design represents in our opinion a more relevant approach as the oxygen treatment was started after a plaque initiation period of 4 weeks and was continued for another 4 weeks. In addition to the differences in study design, also the oxygen concentrations, treatment frequency and total treatment duration varies between the studies. In chapter 2 95\% oxygen was given 1.5h daily for 4 weeks, Altschul et al. used 60\% oxygen 1-3h three days per week for 13 weeks, Kjeldsen et al. administered $28 \%$ oxygen constantly for 10 weeks and Vesselinowitch et al. applied $100 \%$ oxygen for $2 \mathrm{~h}$, five days per week for 10 weeks. Thus, oxygen treatment is 
beneficial in animal models of atherosclerosis regardless of the species and experimental design and oxygen concentration.

Oxygen treatment has also been successfully used in human atherosclerosis. Patients suffering from sleep apnea, evoking intermittent hypoxia, present with advanced atherosclerosis, which is reduced upon oxygen inhalation therapy (8). However, the treatment with oxygen is impractical and not applicable on a larger number of patients. The use of oxygen masks and nasal cannulas may lead to skin irritation, nasal dryness, fatigue and morning tiredness. In addition, oxygen therapy may suppress the breathing reflex in rare cases. The equipment also immobilizes patients, limiting work efficiency and hence be economically unattractive. Finally, increased oxygen concentrations inside of closed rooms raise the fire hazard substantially (9). Thus, the impractical therapeutic circumstances of oxygen therapy without direct beneficial effects for the patient, unlike in breathing disorders, and the potential life-long therapy compliance, seriously limits the use of oxygen in atherosclerosis management. Therefore, chapter $\mathbf{2}$ also aimed to unravel the cellular mechanism of the successful hyperoxic treatment, to allow a potential pharmacological translation. We found that oxygen treatment increased phagocytosis efficiency, which subsequently increased apoptotic cell removal thus preventing necrotic core formation. Mechanistically, hypoxia downregulated the efferocytosis receptor MerTK, resulting in accumulation of dead cells and necrotic core formation. Thus, instead of the oxygen therapy itself drugs enhancing MerTK activity or preventing its cleavage by A disintegrin A metalloprotease 17 (ADAM17) may partially mimic the therapeutic effects of oxygen treatment (10). However, increased ADAM17 mRNA expression has been associated with an atherosclerosis protective phenotype in mice (11). Other possibilities involve the inhibition of the molecular hypoxia signaling cascade, instead of restoring oxygen levels, for instance by targeting the main hypoxia effector HIF1 $\alpha$. At first sight, HIF1 $\alpha$ appears to be an ideal target, as several processes involved in atherosclerosis development/progression are likely HIF1 $\alpha$ mediated: inflammation, angiogenesis and apoptosis (12). Although HIF1 $\alpha$ conceivably has a negative effect on atherosclerosis, its inhibition bares certain risks. The function of HIF1 $\alpha$ seems to differ in various cell types in atherosclerosis, for instance HIF1 $\alpha$ deficiency in endothelial cells reduced plaque size (13), whereas in dendritic cells HIF1 $\alpha$ deficiency increased plaque size (14). Therefore, the outcome of inhibiting HIF1 $\alpha$ is unpredictable, and potentially even harmful. Besides potentially detrimental cell type specific effects of abolished HIF1 $\alpha$ signaling in atherosclerosis, currently available HIF1 $\alpha$ inhibitors act systemically and likely have organ-specific adverse effects. Potentially all ischemic conditions, such as liver ischemia, and ischemic renal disease, may be exacerbated by HIF1 $\alpha$ inhibition (15). Moreover, adverse effects are especially expected on comorbidities in the elderly cardiovascular patient population as inhibition of HIF1 $\alpha$ might lead to severe side effects after a myocardial infarction, particularly when treating atherosclerosis. Several features of the crucial healing process after a myocardial infarction, such as angiogenesis, cellular adaption to hypoxia, and wound healing are dependent on HIF1 $\alpha$ signaling (16-18). To avoid cell type specific and/or organ-specific side effects, celltargeted treatment strategies were recently introduced and offer an appealing solution. Lipid particles loaded with atorvastatin enabled targeted drug delivery exclusively to macrophages (19). The results of chapter 2 also suggest macrophages as a target for a HIF1 $\alpha$ inhibition. However, our group recently showed that myeloid HIF1 $\alpha$ KO had no effect on 
experimental atherosclerosis (14). Thus, modulating the molecular hypoxia response is promising, but needs careful follow up.

\section{Targeting the molecular hypoxia response via prolyl hydroxylase inhibition - one step up in the signaling cascade}

Opposed to the previous section, with the aim of directly limiting hypoxia by restoration of oxygen levels, we were also interested in the effects of stimulating the molecular hypoxia response in atherosclerosis (chapter $\mathbf{3}$ ) in which we tested the hypothesis that aggravation of the cellular hypoxia response via myeloid PHD2 deficiency increases atherosclerosis burden via elevated hypoxia signaling resulting in increased plaque angiogenesis. To increase the molecular hypoxia response the HIF master regulator PHD2 was inhibited in macrophages (20). The expected effects were uncertain, as on the one hand HIF1 $\alpha$ signaling could be beneficial by preparing macrophages for hypoxia, and thereby preventing macrophage apoptosis. On the other hand increasing HIF1 $\alpha$ levels might have detrimental effects on atherosclerosis, as it is associated with promoting inflammation, and induction of angiogenesis. Despite successfully raising HIF1 $\alpha$ levels and observed larger plaques following PHD2 knockdown, angiogenesis was not increased. Although the plaques were larger, PHD2 inhibition augmented plaque stability via an increase in plaque collagen and cap thickness. This suggests that HIF1 $\alpha$ and/or PHD2 have other functions and induce different pathways than those already described. The same holds true for a manipulation of the remaining isoforms of the PHD enzyme: PHD1 and PHD3. A whole body KO of PHD1 unexpectedly led to smaller plaques in LDLr KO mice via lowering plasma cholesterol levels (Marsch et al. unpublished data). In comparison, a hematopoietic KO of PHD3 resulted in a slight increase in plaque size, while hematopoietic PHD1 KO did not alter cholesterol or atherosclerosis (Marsch et al. unpublished data). These differential outcome suggest PHD isoform-specific effects. Indeed it has been shown that the three isoforms have several binding partners inside and outside of the hypoxia signaling cascade (21). However, to date only a limited number of the described molecules have been associated with atherosclerosis: activation transcription factor $4, \beta(2)$-adrenergic receptor and inhibitor of kappa B kinase $\beta$. Inhibition or stimulation of PHD targets previously known and unknown in in hypercholesterolemic PHD KO mice would be particularly interesting. These experiments would help to explain the underlying mechanism of the different atherosclerotic plaque phenotypes observed in the three PHD KO mice.

Although PHD2 inhibition increased plaque size it simultaneously stabilized the plaques, and thus may be important to prevent plaque rupture, there are several challenges and potential dangers for a potential therapeutic translation. A potential detrimental effect of PHD2 inhibition raises from the observation that PHD2 inhibition increased plaque size and alters the cellular metabolism. Preliminary data from our group show that the elevated HIF1 $\alpha$ signaling provokes a metabolic switch thereby increasing glycolysis in macrophages (Figure 1, unpublished). Augmented glycolysis has been linked with increased inflammation via increased tumor necrosis factor $\alpha$ (TNF $\alpha$ ) secretion (HIF1 $\alpha$ dependent) (22). In line, increasing oxygen levels dampened the pro-inflammatory phenotype of M1 macrophages (22) and PHD2 inhibition led to increased TNF $\alpha$ secretion by macrophages (chapter 3 ). Moreover, increased glycolysis also leads to an increase of its waste products, namely lactate. Consequently, excessive lactate accumulation lowers the $\mathrm{pH}$ in the plaque and a 
low $\mathrm{pH}$ has been associated with for instance inhibition and activation of enzyme functionality and induction of apoptosis (23). Besides lactate, also oxidative stress increases with a higher glycolysis rate. In turn, increased oxidative stress may induce macrophage apoptosis (24) and additional lipid oxidation (25) thereby contributing to atherosclerosis progression/severity. Thus, the inhibition of glycolysis may be a possible novel approach. Recently, 6-phosphofructo-2-kinase/fructose-2,6-biphosphatase 3 (PFKFB3) a key enzyme of glycolysis was inhibited in macrophages in vitro. As expected, glycolysis was hampered and simultaneously inflammation was lowered (22). Testing currently available PFKFB3 inhibitors (26) in experimental atherosclerosis would be a relevant and interesting experiment.

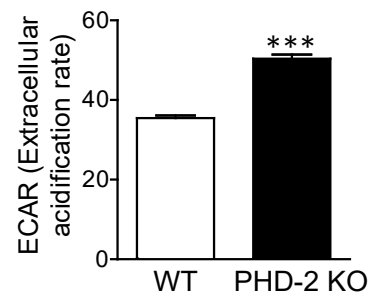

Figure 1. PHD2 KO increases glycolysis in macrophages in vitro (unpublished)

PHD2 KO increases the extracellular acidification rate, a measure of the glycolysis rate, in vitro (WT n=9, PHD2 KO $\mathrm{n}=8$ ). Measured by Seahorse (Agilent). Mean \pm SEM, $p<0.001$

The potential harm of PHD2 inhibition was also demonstrated by the Quebec beer-drinker's myopathy in 1960s presumably resulting from cobalt sulfate, a broad spectrum PHD inhibitor, added to beer by a Canadian beer company (27). In support of this finding, experimental PHD2 inhibition in the murine heart also led to cardiomyopathy (28). Another limitation in the translation of the findings presented in chapter $\mathbf{3}$ is based on the experimental design of the study: LysM-Cre conditional deletion results in PHD2 knockdown in all myeloid cells from conception and is thus already present before atherosclerosis induction. Therefore, the treatment of established atherosclerosis, most relevant for the clinical implications, remains to be investigated.

There are several other potential dangers that urge careful consideration before translating our findings into human disease. Given the effects on plaque size the ongoing clinical trials, in which for instance anemia in patients with chronic kidney disease is treated with PHD inhibitors (NCT02174731), should integrate atherosclerosis as an additional measure. On the one hand potential adverse effects of PHD inhibition on atherosclerosis such as hyperactive angiogenesis (29) or a pro-inflammatory macrophage phenotype (22) might be observed. On the other hand, potential advantageous effects such as increased plaque stability (chapter $\mathbf{3}$ ) and lower plasma cholesterol (Marsch et al. unpublished) could be documented. However, the currently available PHD inhibitors also have limitations, as these are neither isoform specific nor cell type specific and therefore the retrospective evaluation of their effects on atherosclerosis will be challenging.

\section{Angiogenesis research in atherosclerosis - lack of suitable (mouse) models}

In addition to chapter 3, also chapter $\mathbf{4}$ and chapter $\mathbf{5}$ aimed to modulate angiogenesis in murine atherosclerosis by inducing increased neo-angiogenesis (chapter $\mathbf{3}$ ), inducing 
microvascular pericyte loss in a PDGF-B retention motif KO (chapter 4 ) and normalizing microvessels via ant-Ang-2 treatment (chapter 5). However, in all three studies intra-plaque angiogenesis was not observed, nor any effects of the two interventions on plaqueassociated vasa vasorum. Qualitative differences were also not observed. These findings question the suitability of mice to investigate the angiogenesis in atherosclerosis hypothesis. In many aspects, murine plaques are phenotypically comparable to human atherosclerosis, but there are certain important differences as they are much smaller, less fibrotic, more cellular, show virtually no intra-plaque angiogenesis, and only rarely display intra-plaque hemorrhage. However, the most profound difference between mouse and men is the absence of plaque rupture in murine atherosclerosis (30). In addition, the low incidence of intra-plaque hemorrhage already indicates a low incidence of plaque angiogenesis, as red blood cells are presumed to extravasate from hyper-permeable microvessels (31). Numerous studies aimed to quantify and/or manipulate intra-plaque angiogenesis in murine atherosclerosis, however, only very few studies succeeded to do so, as shown in Table $1 a+b$. Studies describing feeding atherosclerosis- prone mice a high cholesterol diet/Western type diet for a relatively short time of 7-25 weeks almost all failed to show intra-plaque angiogenesis (32-40) (Table 1a). Furthermore, the few studies which reported plaque angiogenesis show a very low incidence: $15 \%$ of the mice have intra-plaque microvessels, with a maximum of 1.5 vessels per mouse (36). In comparison human atherosclerotic plaques for example thick fibrous cap atheroma display with around 50 microvessels $/ \mathrm{mm}_{2}$ (41). The remaining studies do not provide microvessel counts or density (MVD) at all. Instead the total amount CD31+ area in the intima is measured (32), or data supporting the stated presence of intra-plaque vessels are not even shown (33). Our own results are in line with these results, as we did not observe any intra-plaque microvessels in three separate studies, while also using a relatively short high cholesterol diet duration of 8-12 weeks (chapter 3, chapter 4 and chapter 5).

Intra-plaque vessels are more frequently observed in atherosclerosis-prone mice aged 4094 weeks $(42,43)$ or after prolonged feeding with a high cholesterol diet/Western type diet (39-96 weeks) (44-49). The incidence of mice with intra-plaque vessels still remains low, ranging from 5 to $30 \%$ of the total analyzed mice (Table 1b). However, no microvessel density or count was provided in these studies.

Besides aging and diet, several other mouse models have been used to induce arthrosclerosis/angiogenesis: stress on a mixed SV/129- C57/BI6 background (50), collar placement (51-56), vein graft (57), stenosis (58) and wire injury (59). Several studies state the presence of intra-plaque vessels, but again do not provide a frequency or microvessel count/density $(50,51,54,56,57)$. Only two studies do provide a microvessel density: 0.03 microvessels per $\mathrm{mm}^{2}(58)$ or 0.5 microvessels per $\mathrm{mm}^{2}$ at baseline, and $1.75 / \mathrm{mm}^{2}$ after treatment (59). This again underlines the very low incidence of intra-plaque microvessels in mice, even in surgery models.

However, out of all listed studies (Table $1 a+b)$ there are two examples showing relevant differences in intra-plaque angiogenesis, also providing an adequate quantification $(60,61)$. Here the frequency of intra-plaque vessels is relatively high compared to control. In Moulton et al., 53\% of ApoE collagen XVIII double KO and $13 \%$ of the control mice displayed with minimum one intra-plaque vessel (60). The frequency was even higher in the study by van der Donckt et al. with 90\% of the ApoE fibrillin double KO mice (MVD 6 vessels $/ 10^{7} \mu \mathrm{m}^{2}$ ) and $4 \%$ of the single KO mice (MVD 0.2 vessels $/ 10^{7} \mu \mathrm{m}^{2}$ ) having at least one microvessel per 
plaque (61). Remarkably, both studies observe the differences in plaque angiogenesis after a KO of extracellular matrix components (Collagen XVIII (60) and fibrillin (61)). Furthermore, the process of angiogenesis largely depends on the cleavage of extracellular matrix by several different proteases (62). Likewise, the density of intra-plaque microvessels was shown to correlate with the expression of matrix metalloproteases in rabbits (63). Correspondingly, Eriksson (44) observed that the number of adventitial vessels is higher in case of medial damage, which also suggests matrix degradation as crucial process for neoangiogenesis. The same connection between medial disruption and increased frequency of intra-plaque microvessels has been shown in human autopsy studies (64). This observation might explain the results described in chapter 3. Here, we aimed to induce a pro-angiogenic plaque macrophage phenotype by myeloid PHD2 deficiency, but simultaneously extensive plaque fibrosis was induced. The excessive fibrosis might have hampered the angiogenic process. However, human plaques are very fibrotic, and yet intra-plaque angiogenesis is frequently observed (30). Besides extracellular matrix degradation, also the different biomechanical properties between mice and man might explain the lack of plaque angiogenesis (65-67). The distinct biomechanical properties, such as less fibrotic material stiffness (cellular and hypocellular), and a fundamental difference in plaque morphology (dome-like) together with a smaller vessel size result in lower residual parietal stress as well as lower peak cap stress in murine compared to human plaques $(67,68)$. These lower mechanical stresses are thought to prevent plaque rupture in mice. In addition, tissue contraction and deformation have been shown to induce VEGF-A expression (69). Lower biomechanical stresses might account for lower VEGF-A levels in mice versus humans. Indeed, ruptured human plaques express higher levels of VEGF-A compared to stable plaques (70). In murine atherosclerosis only experimental overexpression of VEGF-A increased signs of plaque vulnerability (32), showing that autologous VEGF-A expression advanced murine lesions is not sufficient to evoke signs of plaque rupture.

In addition to biomechanical differences between plaques of mice and man, transcriptional regulation may differ. Although angiogenesis is an evolutionary conserved process (71), small differences in transcriptional regulation of the angiogenic process itself could explain the absence of intra-plaque angiogenesis in murine compared to human atherosclerosis. To further investigate the possibility of differences in the angiogenic pathway between mouse and man, the transcriptome of both human and murine primary endothelial cells, after angiogenic priming with for instance VEGF-A could be compared in future studies.

In addition to the molecular, matrix and biomechanical differences, there are fundamental etiological differences between human and murine atherosclerosis: in mice hypercholesterolemia is primarily causing atherosclerosis, whereas in humans multiple risk factors contribute to disease development. Also, progression of atherosclerosis takes several decades in humans and only several weeks in mice. Thus atherosclerosis is modeled in relatively young animals, whereas patients are often elderly (30).

Overall, a minority of the studies intending to investigate intra-plaque angiogenesis in murine atherosclerosis have delivered the anticipated result to mimic or manipulate intraplaque angiogenesis (table 1). Therefore, the majority of these studies instead analyzed the effects of the desired intervention on the plaque associated vasa vasorum of the adventitia, as surrogate for intra-plaque microvessels (including chapter 3, chapter 4 and chapter 5). The adventitial vasa vasorum have a crucial role in the development of atherosclerosis in humans, as the largest proportion of the intra-plaque vessels derives from the adventitia 
and very rarely from the lumen (72). Nevertheless, a direct involvement of the adventitia in plaque rupture is unlikely. Effects may be more indirect as inflammatory cells and angiogenic factors, as well as intra-plaque vessels are thought to originate from the adventitia (73). However, in humans, plaque rupture has been linked with increased intraplaque angiogenesis rather than an increase in adventitial vasa vasorum. More research is needed to clarify a possible connection between vasa vasorum density and rupture susceptibility in humans. Thus far, this limits the impact of the obtained results from murine studies using adventitial angiogenesis as an outcome parameter. Besides this major biological limitation, several methodological limitations persist that impede the comparability of the performed studies and future use. The outcome is scarcely comparable, as atherosclerosis was studied in various locations: aortic root, ascending aorta, descending aorta, brachiocephalic artery, carotid artery etc. In addition to the different locations, the data presentation highly varies among the studies: microvessel density (no. of microvessels per $\mathrm{mm}^{2}$ ), microvessel count (per section or per mouse), CD31 positive adventitial area or vasa vasorum volume were used (Table $1 a+b)$. Moreover, also the imaging method varied: the majority of studies used histology, but also intra-vital microscopy, two photon microscopy, confocal microscopy and micro CT have been used to visualize adventitial microvessels (Table $1 a+b)$. The experimental design also limits the translatability of the findings. In two studies, induction/manipulation of angiogenesis was started together with atherosclerosis induction $(39,45)$, whereas pre-existing plaques represent the treatment target in human atherosclerosis. The same is true for KO models: the $\mathrm{KO}$ is present prior to atherosclerosis initiation. Similarly, side effects of the chosen treatments were either not determined $(49,74)$, or had significant effects on other cells/processes involved in atherosclerosis development (52-54). Thus, the effect of a certain treatment on atherosclerosis size or complexity may not be exclusively attributed to changes in angiogenesis. Especially, since microvessel numbers itself correlated with plaque size and complexity (44). Because of the ambivalence of the used compounds, conclusions regarding microvessel normalization - so improving microvessel quality rather than reducing quantity - are difficult to derive from the listed studies. Agents that potentially normalize microvessel functionality, are often also anti-angiogenic, as for instance anti-VEGF treatment (75). Additionally microvessel quality was assessed in very few studies only (chapter 3, chapter 4, $(42,61)$ ).

Overall, the studies listed in table $1 \mathrm{a}+\mathrm{b}$ still give valuable insights for the understanding of atherosclerosis, by investigating possible effects of pro- and anti-angiogenic treatment on atherosclerosis and existing co-morbidities. VEGF-A gene therapy, which might be used to induce angiogenesis in peripheral artery disease (76), increased experimental atherosclerosis $(32,54)$. However, the effect was not mediated by increased plaque angiogenesis, instead VEGF-A increased leukocyte recruitment without affecting plaque angiogenesis (54). Chapter 5 where we initially hypothesized that anti-Ang-2 treatment would normalize existing microvessels and thereby reduce atherosclerosis burden in $\mathrm{ApoB}^{100 / 100}$ LDLr KO mice, still provides valuable insights despite the lack of effect. The inhibition of vascular growth factors is frequently used in oncology and increasing numbers of patients survive cancer therapy. Therefore evaluating the risk of Ang-2 inhibition on atherosclerosis as a frequent co-morbidity of elder cancer patients is important. We found that anti-Ang-2 treatment act beneficial, rather than harmful with respect to atherosclerosis, as it reduced plaque initiation, likely via the reduction of plasma triglyceride 
levels. This finding may be significant as high triglyceride levels contribute importantly to human atherosclerosis development and burden (77).

Chapter $\mathbf{4}$ is another example of a study that failed, due to the lack of angiogenesis, as we initially hypothesized that microvascular pericyte loss via PDGF-B retention motif $\mathrm{KO}$ and subsequent increased microvascular permeability would exacerbate atherosclerosis. Still, it provided valuable insights for atherosclerosis research. We found that PDGF-B retention motif KO in atherosclerosis resulted in a pro-inflammatory leukocyte profile and elevated plaque fibrosis thereby increasing plaque size. Thus, PDGF-B is likely not a suitable target for atherosclerosis therapy, as it may have opposing effects. To make use of the potential benefits of PDGF-B inhibition or stimulation its downstream pathways should be targeted to avoid unwanted side effects.

Almost all aforementioned studies that intended to quantify and/or manipulate angiogenesis in atherosclerosis have two major things in common, first the use of mice as model organisms, and second an intervention strategy aimed at antiangiogenic molecules or peptides, (thalidomide (45), TNP-470 (49), angiostatin $(48,78)$, etc.) or inhibition of vascular growth factors (anti-Ang-2 (chapter 5), anti-VEGF-A (79)) to reduce microvessel numbers. A similar strategy has been applied to induce angiogenesis (FGF (43), VEGF-A (32) etc.). However, the scope of these strategies might be too narrow and novel approaches should be included in the future to broaden the view beyond vascular growth factors. Several pro- and anti-angiogenic microRNAs (miRNA) have been described in the recent years (80). These could for instance potentially be used to manage angiogenesis in atherosclerosis. Furthermore, angiogenic endothelial and pericyte precursors haven been found in the adventitia $(73,81)$. Possibly, stimulation of these progenitor cells could be used to prevent hyperactive intra-plaque angiogenesis or to increase pericyte coverage of present leaky intra-plaque vessels.

Together, literature suggests mouse models to be unsuitable for the investigation of angiogenesis in atherosclerosis, thus warranting the use of other animal models. So far rats (Table 2), rabbits (Table 2), pigs (Table 3 ) and non-human primates have been used to study angiogenesis in atherosclerosis. In rabbit models, atherosclerosis was mostly induced by a combination of balloon angioplasty and high cholesterol diet, leading to plaques with a baseline MVD between 15 and 80 vessels per $\mathrm{mm}^{2}$ (Table 2 ). In some studies, adventitial angiogenesis was specifically targeted using a hollow perivascular collar together with a relatively short post-operation time of 9 to 21 days (82-85). In addition to rabbits, pro- and anti-angiogenic treatments were also applied in pig models of atherosclerosis (Table 3 ). In pigs, atherosclerosis was induced by high cholesterol diet and/or surgical interventions (balloon angioplasty or stenting). However, intra-plaque angiogenesis was absent in all analyzed studies except for one (Table 3). Here, a genetically engineered Yucatan mini pig was used, which develops hypercholesterolemia due to pro-protein convertase subtilisin/kexin type 9 (PCSK9) overexpression, when fed a high cholesterol diet (86). The resulting plaques show a human like morphology including intra-plaque and adventitial angiogenesis. However, data on MVD were unfortunately not provided. Practically, larger animal models allow the use of clinical diagnostic tools such as magnetic resonance imaging to detect microvessels. Therefore it will be easier to translate the study results to the human situation. To conclude, mice are a good model for atherosclerosis research in general, but fall short when aiming at plaque angiogenesis. This should preferably be studied in larger animal models such as the recently developed PCSK9 overexpressing pig model. 


\section{Potential side effects in pro-angiogenic therapy}

Similar to the previously described approach of evoking microvessel formation in atherosclerotic mice by using pro-angiogenic factors such VEGFs, the use of pro-angiogenic factors has many possible applications in cardiovascular therapy (87). For instance, VEGF-A gene therapy has been tested for the treatment of peripheral artery disease (76). Besides the induction of angiogenesis, VEGF-A gene therapy was reported to induce increased vascular permeability, and even edema formation (88). In chapter 6 in which we compared the effects of histamine, and VEGF-A, D and $F$ on the angiogenic response and microvascular permeability, and confirmed this effect. Additionally the endothelial transcriptomic response to VEGF-A and histamine was compared. We found that histamine and VEGF-A amongst others commonly downregulate claudin-5 which is important for endothelial tight junction formation and thus permeability and upregulate endothelial cell proliferation necessary for angiogenesis. We further identified that these processes are regulated via Nmyc proto-oncogene protein (MYCN) and nuclear receptor subfamily 4 group A member 1 (NR4A1). Thus, inhibition of inhibition of MYCN signaling by for instance CDK7 inhibitors (89) or NR4A1 signaling by for instance calcineurin inhibitors (90) may reduce edema formation in pro-angiogenic gene therapy. Our identification of other commonly and independently regulated permeability inducing pathways by VEGF-A and histamine might help to prevent side effects of pro-angiogenic gene therapy in the future.

\section{Concluding Remarks}

Plaque hypoxia and plaque angiogenesis are thought to be substantial contributors to plaque rupture in humans. However, this ides is based mainly on human autopsy studies and a causal relation has not yet been established. Moreover, as the data presented in this thesis show, manipulating plaque hypoxia and angiogenesis in experimental atherosclerosis to understand the underlying mechanisms and causal relation to plaque rupture is challenging.

Although plaque hypoxia was reversible and causally related to atherogenesis, more research is needed to translate this systemic treatment into a molecular therapy. Our research showed that attempts to modulate the hypoxic signaling cascade need careful evaluation to detect and exclude unwanted side effects. However, unexpected findings may provide novel insights, as enhanced hypoxia signaling by PHD2 cKO improved plaque stabilization.

Angiogenesis in atherosclerosis is difficult to study in mouse models of the disease, as murine plaques lack microvessels, adventitial microvessels are a poor surrogate of intraplaque vessels and experiments increasing microvessel numbers or altering microvessel quality including those described in this thesis have failed. The limitations probably arise from dissimilarities with the human plaque stemming from molecular, matrix, biomechanical, transcriptional and/or etiological differences between species. However, interference with the vascular growth factor Ang-2 in experimental atherosclerosis showed that a treatment with anti-Ang-2 therapy is likely not harmful from a cardiovascular point of view. In comparison, manipulation of the vascular growth factor PDGF-B is rather unfavorable for atherosclerosis treatment. Moreover, safety of using VEGFs for proangiogenic therapy could be improved by considering the identified downstream pathways inducing permeability in endothelial cells. 


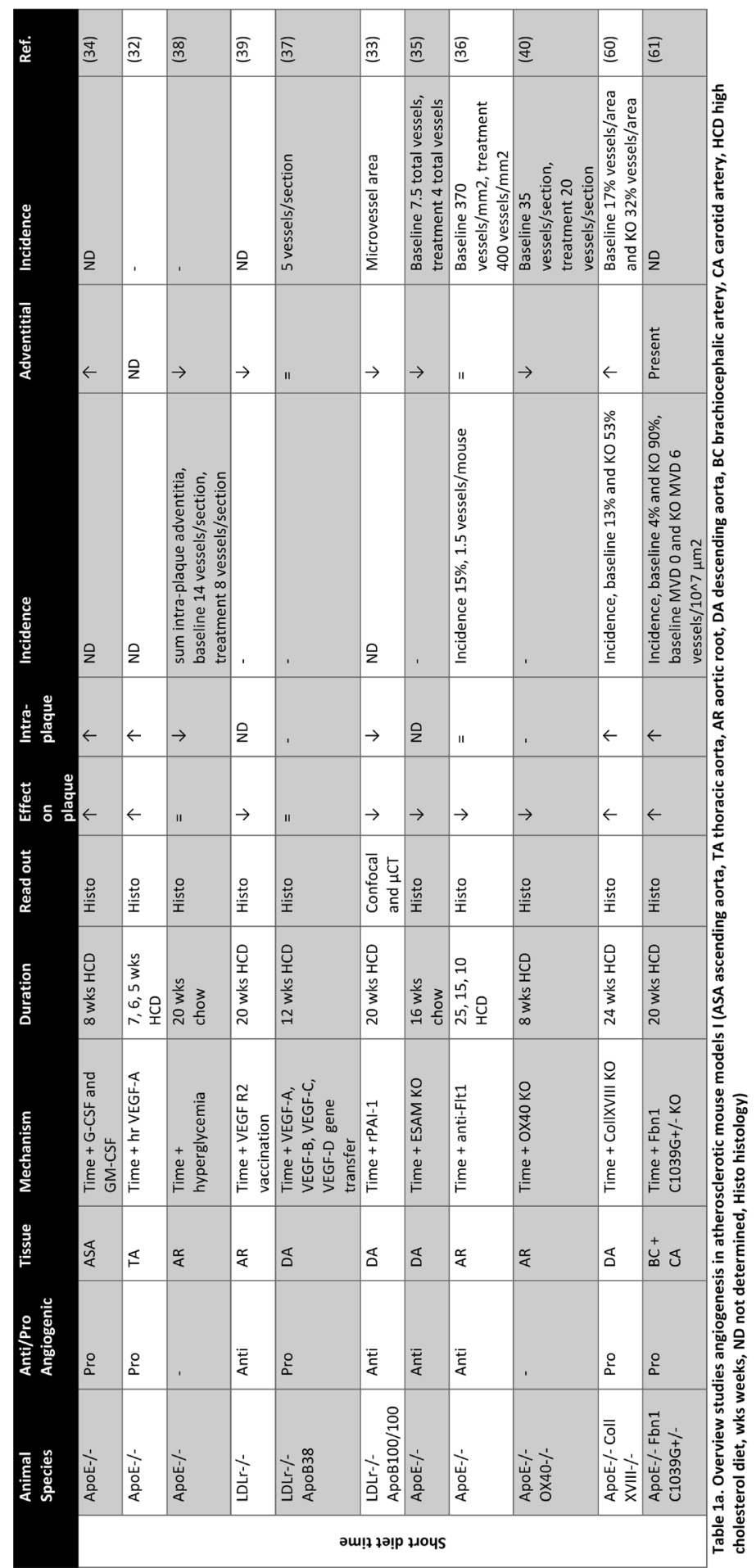




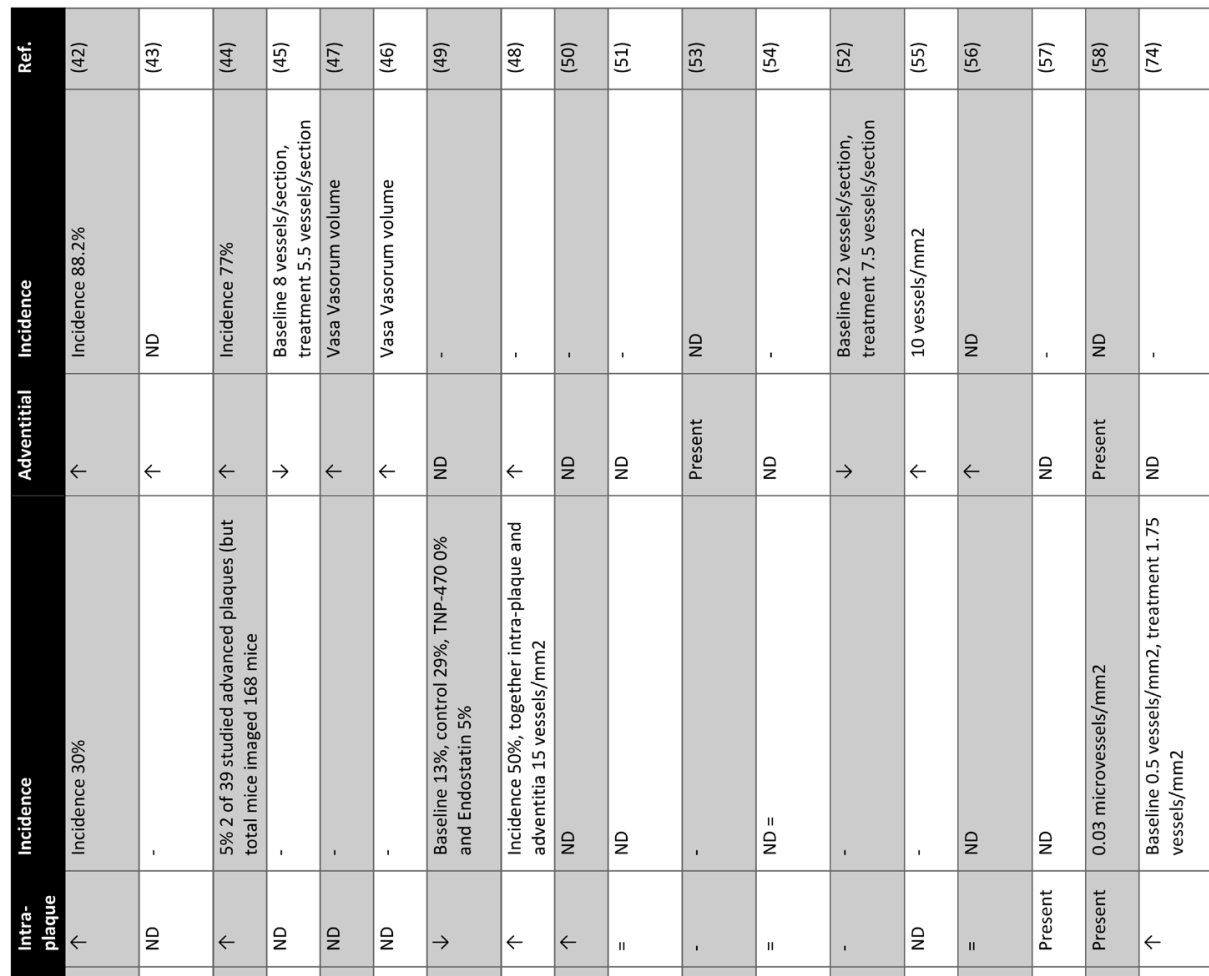

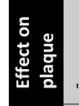

言

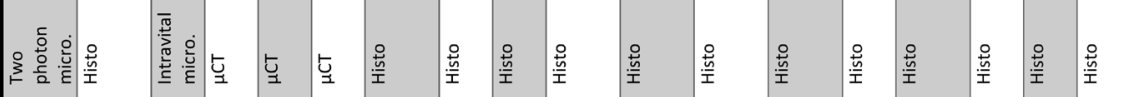

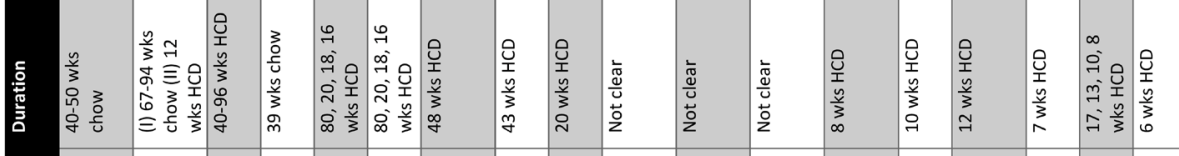

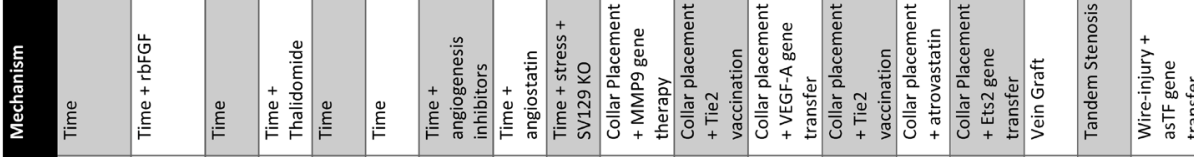
耪

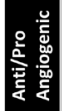

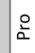

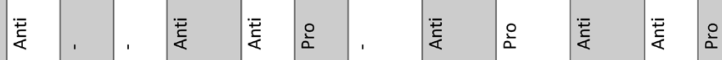
을

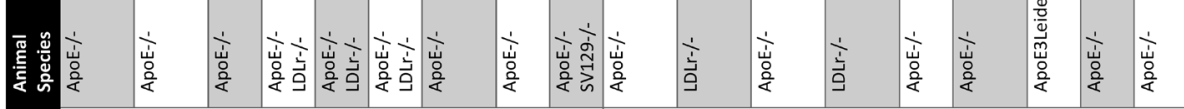




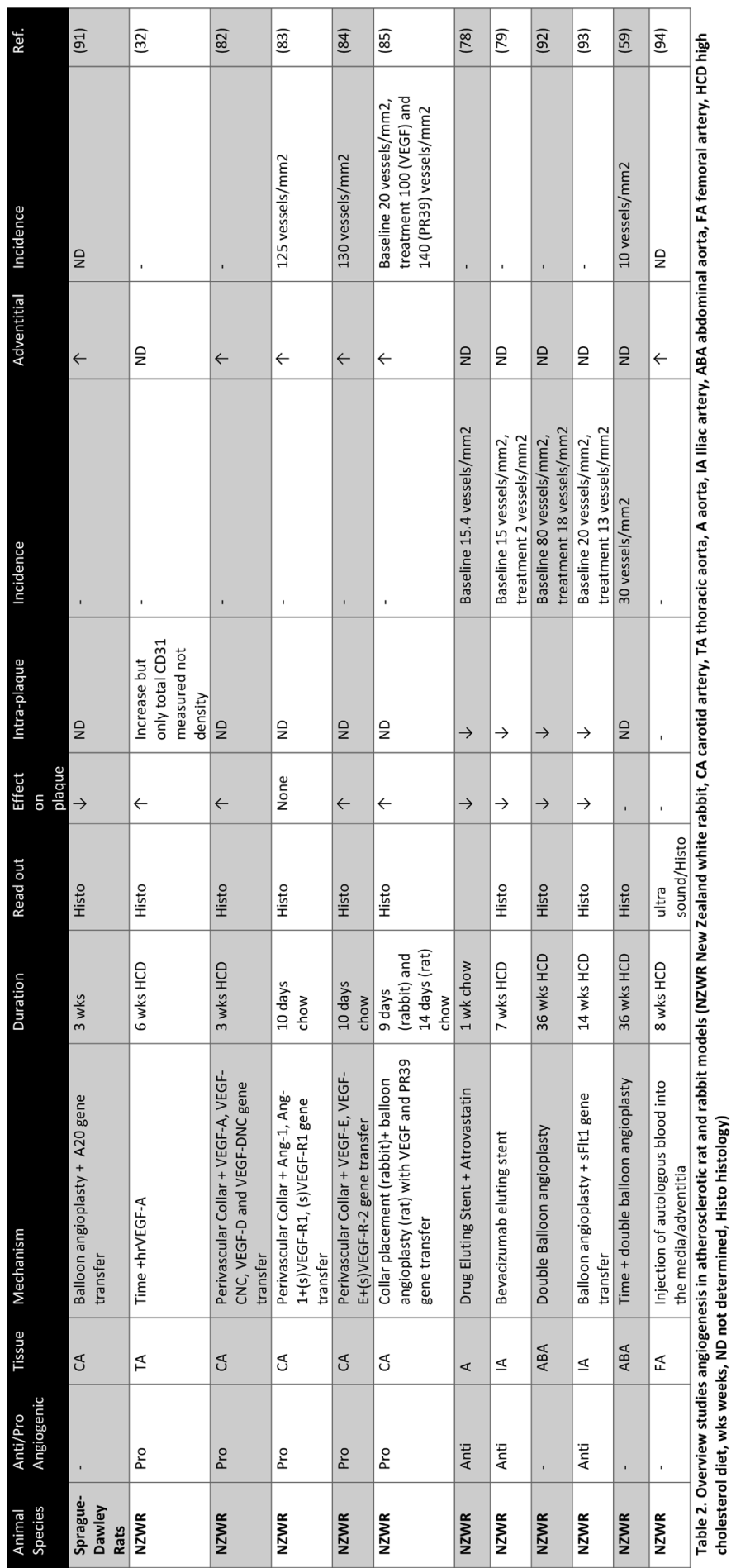


Chapter 7

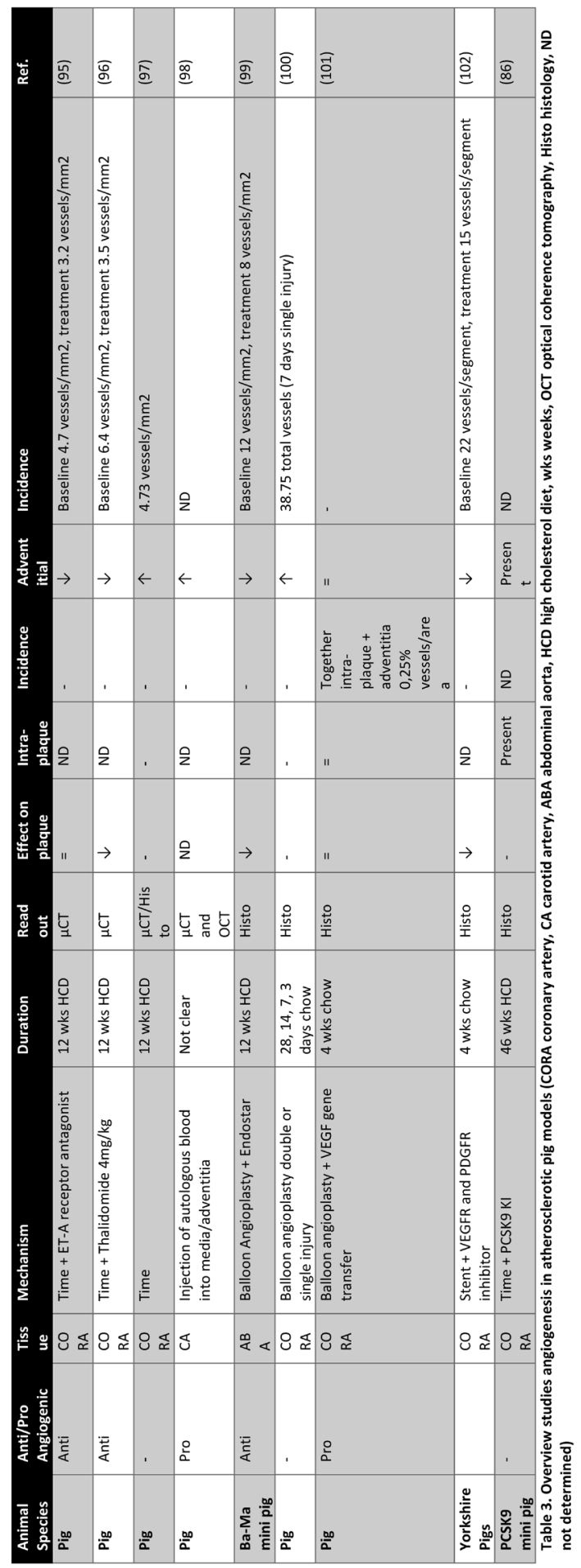




\section{References}

1. Sluimer JC, Gasc J-M, van Wanroij JL, Kisters N, Groeneweg M, Sollewijn Gelpke MD, et al. Hypoxia, hypoxia-inducible transcription factor, and macrophages in human atherosclerotic plaques are correlated with intraplaque angiogenesis. J Am Coll Cardiol. 2008 Apr 1;51(13):1258-65.

2. Hueper WC. The anoxemia theory. Arch Path. 1944;39:162, 245, 350.

3. ALTSCHUL R, HERMAN IH. Influence of oxygen inhalation on cholesterol metabolism. Arch Biochem Biophys. 1954 Jul;51(1):308-9.

4. Kjeldsen K, Astrup P, Wanstrup J. Reversal of rabbit atheromatosis by hyperoxia. J Atheroscler Res. $1969 ; 10(2): 173-8$.

5. Okamoto R, Hatani M, Tsukitani M, Suehiro A, Fujino M, Imai N, et al. The effect of oxygen on the development of atherosclerosis in WHHL rabbits. Atherosclerosis. $1983 \mathrm{Apr}$;47(1):47-53.

6. Vesselinovitch D, Wissler RW, Fisher-Dzoga K, Hughes R, Dubien L. Regression of atherosclerosis in rabbits. I. Treatment with low-fat diet, hyperoxia and hypolipidemic agents. Atherosclerosis. 1974 Mar;19(2):259-75.

7. Shim J, Al-Mashhadi RH, Sørensen CB, Bentzon JF. Large animal models of atherosclerosis - new tools for persistent problems in cardiovascular medicine. J Pathol. 2015 Sep 28;914531200.

8. Drager LF, Bortolotto LA, Figueiredo AC, Krieger EM, Lorenzi GF. Effects of continuous positive airway pressure on early signs of atherosclerosis in obstructive sleep apnea. Am J Respir Crit Care Med. 2007 Oct 1;176(7):706-12.

9. NIH. What Is Oxygen Therapy? [Internet]. 2012 [cited 2015 Dec 11]. Available from: http://www.nhlbi.nih.gov/health/health-topics/topics/oxt

10. Thorp E, Vaisar T, Subramanian M, Mautner L, Blobel C, Tabas I. Shedding of the Mer tyrosine kinase receptor is mediated by ADAM17 protein through a pathway involving reactive oxygen species, protein kinase $C \delta$, and p38 mitogen-activated protein kinase (MAPK). J Biol Chem. 2011 Sep 23;286(38):3333544.

11. Holdt LM, Thiery J, Breslow JL, Teupser D. Increased ADAM17 mRNA Expression and Activity Is Associated With Atherosclerosis Resistance in LDL-Receptor Deficient Mice. Arterioscler Thromb Vasc Biol. 2008 Jun 1;28(6):1097-103.

12. Gao L, Chen Q, Zhou X, Fan L. The role of hypoxia-inducible factor 1 in atherosclerosis. J Clin Pathol. 2012 Oct;65(10):872-6.

13. Akhtar S, Hartmann P, Karshovska E, Rinderknecht F-A, Subramanian P, Gremse F, et al. Endothelial Hypoxia-Inducible Factor-1 $\alpha$ Promotes Atherosclerosis and Monocyte Recruitment by Upregulating MicroRNA-19a. Hypertension. 2015 Oct 19;HYPERTENSIONAHA.115.05886.

14. Chaudhari SM, Sluimer JC, Koch M, Theelen TL, Manthey HD, Busch M, et al. Deficiency of HIF1 $\alpha$ in Antigen-Presenting Cells Aggravates Atherosclerosis and Type 1 T-Helper Cell Responses in Mice. Arterioscler Thromb Vasc Biol. 2015 Nov;35(11):2316-25.

15. Semenza GL. Hypoxia-inducible factors: mediators of cancer progression and targets for cancer therapy. Trends Pharmacol Sci. 2012 Apr;33(4):207-14.

16. Ockaili R, Natarajan R, Salloum F, Fisher BJ, Jones D, Fowler AA, et al. HIF-1 activation attenuates postischemic myocardial injury: role for heme oxygenase-1 in modulating microvascular chemokine generation. Am J Physiol Heart Circ Physiol. 2005 Aug;289(2):H542-8.

17. Huang M, Nguyen P, Jia F, Hu S, Gong Y, de Almeida PE, et al. Double knockdown of prolyl hydroxylase 
and factor-inhibiting hypoxia-inducible factor with nonviral minicircle gene therapy enhances stem cell mobilization and angiogenesis after myocardial infarction. Circulation. 2011 Sep 13;124(11 Suppl):S4654.

18. Shyu KG, Wang MT, Wang BW, Chang CC, Leu JG, Kuan P, et al. Intramyocardial injection of naked DNA encoding HIF-1alpha/VP16 hybrid to enhance angiogenesis in an acute myocardial infarction model in the rat. Cardiovasc Res. 2002 Jun;54(3):576-83.

19. Tang J, Lobatto ME, Hassing L, van der Staay S, van Rijs SM, Calcagno C, et al. Inhibiting macrophage proliferation suppresses atherosclerotic plaque inflammation. Sci Adv. 2015 Apr;1(3).

20. Bishop T, Ratcliffe PJ. HIF hydroxylase pathways in cardiovascular physiology and medicine. Circ Res. 2015 Jun 19;117(1):65-79.

21. Wong BW, Kuchnio A, Bruning U, Carmeliet P. Emerging novel functions of the oxygen-sensing prolyl hydroxylase domain enzymes. Trends Biochem Sci. Elsevier Ltd; 2013 Jan;38(1):3-11.

22. Tawakol A, Singh P, Mojena M, Pimentel-Santillana M, Emami H, MacNabb M, et al. HIF-1 $\alpha$ and PFKFB3 Mediate a Tight Relationship Between Proinflammatory Activation and Anerobic Metabolism in Atherosclerotic Macrophages. Arterioscler Thromb Vasc Biol. 2015 Jun;35(6):1463-71.

23. Naghavi M, John R, Naguib S, Siadaty MS, Grasu R, Kurian KC, et al. pH Heterogeneity of human and rabbit atherosclerotic plaques; a new insight into detection of vulnerable plaque. Atherosclerosis. 2002 Sep;164(1):27-35.

24. Moore KJ, Tabas I. Macrophages in the pathogenesis of atherosclerosis. Cell. Elsevier; 2011 Apr 29;145(3):341-55.

25. Stocker R, Keaney JF. Role of oxidative modifications in atherosclerosis. Physiol Rev. 2004 Oct;84(4):1381-478.

26. Clem BF, O’Neal J, Tapolsky G, Clem AL, Imbert-Fernandez Y, Kerr DA, et al. Targeting 6-phosphofructo2-kinase (PFKFB3) as a therapeutic strategy against cancer. Mol Cancer Ther. 2013 Aug;12(8):1461-70.

27. Morin Y, Daniel P. Quebec beer-drinkers' cardiomyopathy: etiological considerations. Can Med Assoc J. 1967 Oct 7;97(15):926-8.

28. Moslehi J, Minamishima YA, Shi J, Neuberg D, Charytan DM, Padera RF, et al. Loss of hypoxia-inducible factor prolyl hydroxylase activity in cardiomyocytes phenocopies ischemic cardiomyopathy. Circulation. 2010 Sep 7;122(10):1004-16.

29. Takeda K, Cowan A, Fong G-H. Essential role for prolyl hydroxylase domain protein 2 in oxygen homeostasis of the adult vascular system. Circulation. 2007 Aug 14;116(7):774-81.

30. Bentzon JF, Falk E. Atherosclerotic lesions in mouse and man: is it the same disease? Curr Opin Lipidol. 2010 Oct;21(5):434-40.

31. Michel J-B, Virmani R, Arbustini E, Pasterkamp G. Intraplaque haemorrhages as the trigger of plaque vulnerability. Eur Heart J. 2011 Aug;32(16):1977-85, 1985a, 1985b, 1985c.

32. Celletti FL, Waugh JM, Amabile PG, Brendolan A, Hilfiker PR, Dake MD. Vascular endothelial growth factor enhances atherosclerotic plaque progression. Nat Med. 2001 Apr;7(4):425-9.

33. Drinane M, Mollmark J, Zagorchev L, Moodie K, Sun B, Hall A, et al. The antiangiogenic activity of rPAI$1(23)$ inhibits vasa vasorum and growth of atherosclerotic plaque. Circ Res. 2009 Feb 13;104(3):337-45.

34. Haghighat A, Weiss D, Whalin MK, Cowan DP, Taylor WR. Granulocyte colony-stimulating factor and granulocyte macrophage colony-stimulating factor exacerbate atherosclerosis in apolipoprotein Edeficient mice. Circulation. 2007 Apr 17;115(15):2049-54.

35. Inoue M, Ishida T, Yasuda T, Toh R, Hara T, Cangara HM, et al. Endothelial cell-selective adhesion 
molecule modulates atherosclerosis through plaque angiogenesis and monocyte-endothelial interaction. Microvasc Res. Elsevier Inc.; 2010 Sep;80(2):179-87.

36. Luttun A, Tjwa M, Moons L, Wu Y, Angelillo-Scherrer A, Liao F, et al. Revascularization of ischemic tissues by PIGF treatment, and inhibition of tumor angiogenesis, arthritis and atherosclerosis by anti-Flt1. Nat Med. 2002 Aug;8(8):831-40.

37. Leppänen $\mathrm{P}$, Koota S, Kholová I, Koponen J, Fieber C, Eriksson U, et al. Gene transfers of vascular endothelial growth factor-A, vascular endothelial growth factor-B, vascular endothelial growth factor-C, and vascular endothelial growth factor-D have no effects on atherosclerosis in hypercholesterolemic low-density lipoprotein. Circulation. 2005 Aug 30;112(9):1347-52.

38. Veerman KJ, Venegas-Pino DE, Shi Y, Khan MI, Gerstein HC, Werstuck GH. Hyperglycaemia is associated with impaired vasa vasorum neovascularization and accelerated atherosclerosis in apolipoprotein-E deficient mice. Atherosclerosis. Elsevier Ltd; 2013 Apr;227(2):250-8.

39. Petrovan RJ, Kaplan CD, Reisfeld RA, Curtiss LK. DNA vaccination against VEGF receptor 2 reduces atherosclerosis in LDL receptor-deficient mice. Arterioscler Thromb Vasc Biol. 2007 May;27(5):1095-100.

40. Nakano M, Fukumoto Y, Satoh K, Ito Y, Kagaya Y, Ishii N, et al. OX40 ligand plays an important role in the development of atherosclerosis through vasa vasorum neovascularization. Cardiovasc Res. 2010 Dec 1;88(3):539-46.

41. Sluimer JC, Kolodgie FD, Bijnens APJJ, Maxfield K, Pacheco E, Kutys B, et al. Thin-walled microvessels in human coronary atherosclerotic plaques show incomplete endothelial junctions relevance of compromised structural integrity for intraplaque microvascular leakage. J Am Coll Cardiol. 2009 Apr 28;53(17):1517-27.

42. Rademakers T, Douma K, Hackeng TM, Post MJ, Sluimer JC, Daemen MJAP, et al. Plaque-associated vasa vasorum in aged apolipoprotein E-deficient mice exhibit proatherogenic functional features in vivo. Arterioscler Thromb Vasc Biol. 2013 Feb;33(2):249-56.

43. Tanaka K, Nagata D, Hirata Y, Tabata Y, Nagai R, Sata M. Augmented angiogenesis in adventitia promotes growth of atherosclerotic plaque in apolipoprotein E-deficient mice. Atherosclerosis. Elsevier Ireland Ltd; 2011 Apr;215(2):366-73.

44. Eriksson EE. Intravital microscopy on atherosclerosis in apolipoprotein e-deficient mice establishes microvessels as major entry pathways for leukocytes to advanced lesions. Circulation. 2011 Nov 8;124(19):2129-38.

45. Kampschulte M, Gunkel I, Stieger P, Sedding DG, Brinkmann A, Ritman EL, et al. Thalidomide influences atherogenesis in aortas of ApoE(-/-)/LDLR (-/-) double knockout mice: a nano-CT study. Int J Cardiovasc Imaging. 2014 Apr;30(4):795-802.

46. Langheinrich AC, Michniewicz A, Bohle RM, Ritman EL. Vasa vasorum neovascularization and lesion distribution among different vascular beds in ApoE-/-/LDL-/- double knockout mice. Atherosclerosis. 2007 Mar;191(1):73-81.

47. Langheinrich AC, Michniewicz A, Sedding DG, Walker G, Beighley PE, Rau WS, et al. Correlation of vasa vasorum neovascularization and plaque progression in aortas of apolipoprotein $E(-/-) /$ low-density lipoprotein(-/-) double knockout mice. Arterioscler Thromb Vasc Biol. 2006 Feb;26(2):347-52.

48. Moulton KS, Vakili K, Zurakowski D, Soliman M, Butterfield C, Sylvin E, et al. Inhibition of plaque neovascularization reduces macrophage accumulation and progression of advanced atherosclerosis. Proc Natl Acad Sci U S A. 2003 Apr 15;100(8):4736-41.

49. Moulton KS, Heller E, Konerding M a, Flynn E, Palinski W, Folkman J. Angiogenesis inhibitors endostatin or TNP-470 reduce intimal neovascularization and plaque growth in apolipoprotein E-deficient mice. Circulation. 1999 Apr 6;99(13):1726-32. 
50. Najafi AH, Aghili N, Tilan JU, Andrews JA, Peng X, Lassance-Soares RM, et al. A new murine model of stress-induced complex atherosclerotic lesions. Dis Model Mech. 2013 Mar;6(2):323-31.

51. de Nooijer R, Verkleij CJN, von der Thüsen JH, Jukema JW, van der Wall EE, van Berkel TJC, et al. Lesional overexpression of matrix metalloproteinase- 9 promotes intraplaque hemorrhage in advanced lesions but not at earlier stages of atherogenesis. Arterioscler Thromb Vasc Biol. 2006 Feb;26(2):340-6.

52. Hauer AD, Habets KLL, van Wanrooij EJA, de Vos P, Krueger J, Reisfeld RA, et al. Vaccination against TIE2 reduces atherosclerosis. Atherosclerosis. 2009 Jun;204(2):365-71.

53. Hauer AD, van Puijvelde GHM, Peterse N, de Vos P, van Weel V, van Wanrooij EJA, et al. Vaccination against VEGFR2 attenuates initiation and progression of atherosclerosis. Arterioscler Thromb Vasc Biol. 2007 Sep;27(9):2050-7.

54. Lucerna M, Zernecke A, de Nooijer R, de Jager SC, Bot I, van der Lans C, et al. Vascular endothelial growth factor-A induces plaque expansion in ApoE knock-out mice by promoting de novo leukocyte recruitment. Blood. 2007 Jan 1;109(1):122-9.

55. Bot I, Jukema JW, Lankhuizen IM, van Berkel TJC, Biessen EAL. Atorvastatin inhibits plaque development and adventitial neovascularization in ApoE deficient mice independent of plasma cholesterol levels. Atherosclerosis. Elsevier Ireland Ltd; 2011 Feb;214(2):295-300.

56. Cheng C, Tempel D, Den Dekker WK, Haasdijk R, Chrifi I, Bos FL, et al. Ets2 determines the inflammatory state of endothelial cells in advanced atherosclerotic lesions. Circ Res. 2011 Aug 5;109(4):382-95.

57. de Vries MR, Niessen HWM, Löwik CWGM, Hamming JF, Jukema JW, Quax PHA. Plaque Rupture Complications in Murine Atherosclerotic Vein Grafts Can Be Prevented by TIMP-1 Overexpression. Schulz C, editor. PLoS One. 2012 Oct 11;7(10):e47134.

58. Chen Y-C, Bui AV, Diesch J, Manasseh R, Hausding C, Rivera J, et al. A novel mouse model of atherosclerotic plaque instability for drug testing and mechanistic/therapeutic discoveries using gene and microRNA expression profiling. Circ Res. 2013 Jul 19;113(3):252-65.

59. Giannarelli C, Ibanez B, Cimmino G, Garcia Ruiz JM, Faita F, Bianchini E, et al. Contrast-enhanced ultrasound imaging detects intraplaque neovascularization in an experimental model of atherosclerosis. JACC Cardiovasc Imaging. Elsevier Inc.; 2010 Dec;3(12):1256-64.

60. Moulton KS, Olsen BR, Sonn S, Fukai N, Zurakowski D, Zeng X. Loss of collagen XVIII enhances neovascularization and vascular permeability in atherosclerosis. Circulation. 2004 Sep 7;110(10):13306.

61. Van der Donckt C, Van Herck JL, Schrijvers DM, Vanhoutte G, Verhoye M, Blockx I, et al. Elastin fragmentation in atherosclerotic mice leads to intraplaque neovascularization, plaque rupture, myocardial infarction, stroke, and sudden death. Eur Heart J. 2015 May 1;36(17):1049-58.

62. Carmeliet P, Jain RK. Molecular mechanisms and clinical applications of angiogenesis. Nature. 2011 May 19;473(7347):298-307.

63. Liu XQ, Mao Y, Wang B, Lu XT, Bai WW, Sun YY, et al. Specific matrix metalloproteinases play different roles in intraplaque angiogenesis and plaque instability in rabbits. Hagemeyer CE, editor. PLoS One. 2014 Sep 18;9(9):e107851.

64. Virmani R, Kolodgie FD, Burke AP, Finn A V, Gold HK, Tulenko TN, et al. Atherosclerotic plaque progression and vulnerability to rupture: angiogenesis as a source of intraplaque hemorrhage. Arterioscler Thromb Vasc Biol. 2005 Oct;25(10):2054-61.

65. Campbell IC, Weiss D, Suever JD, Virmani R, Veneziani A, Vito RP, et al. Biomechanical modeling and morphology analysis indicates plaque rupture due to mechanical failure unlikely in atherosclerosis-prone mice. Am J Physiol Heart Circ Physiol. 2013 Feb 1;304(3):H473-86. 
66. Riou LM, Broisat A, Ghezzi C, Finet G, Rioufol G, Gharib AM, et al. Effects of mechanical properties and atherosclerotic artery size on biomechanical plaque disruption - mouse vs. human. J Biomech. Elsevier; 2014 Mar 3;47(4):765-72.

67. van der Heiden K, Hoogendoorn A, Daemen MJ, Gijsen FJH. Animal models for plaque rupture: a biomechanical assessment. Thromb Haemost. 2015 Nov 26;115(3)

68. Ohayon J, Mesnier N, Broisat A, Toczek J, Riou L, Tracqui P. Elucidating atherosclerotic vulnerable plaque rupture by modeling cross substitution of ApoE-/- mouse and human plaque components stiffnesses. Biomech Model Mechanobiol. 2012 Jul;11(6):801-13.

69. Rivron NC, Vrij EJ, Rouwkema J, Le Gac S, van den Berg A, Truckenmüller RK, et al. Tissue deformation spatially modulates VEGF signaling and angiogenesis. Proc Natl Acad Sci U S A. 2012 May 1;109(18):688691.

70. Pelisek J, Well G, Reeps C, Rudelius M, Kuehnl A, Culmes M, et al. Neovascularization and angiogenic factors in advanced human carotid artery stenosis. Circ J. 2012;76(5):1274-82.

71. Carmeliet P, Tessier-Lavigne M. Common mechanisms of nerve and blood vessel wiring. Nature. 2005 Jul 14;436(7048):193-200.

72. Kumamoto M, Nakashima Y, Sueishi K. Intimal neovascularization in human coronary atherosclerosis: its origin and pathophysiological significance. Hum Pathol. 1995 Apr;26(4):450-6.

73. Stenmark KR, Yeager ME, El Kasmi KC, Nozik-Grayck E, Gerasimovskaya E V, Li M, et al. The adventitia: essential regulator of vascular wall structure and function. Annu Rev Physiol. 2013 Feb 10;75(1):23-47.

74. Giannarelli C, Alique M, Rodriguez DT, Yang DK, Jeong D, Calcagno C, et al. Alternatively spliced tissue factor promotes plaque angiogenesis through the activation of hypoxia-inducible factor- $1 \alpha$ and vascular endothelial growth factor signaling. Circulation. 2014 Oct 7;130(15):1274-86.

75. O'Connor JPB, Carano RAD, Clamp AR, Ross J, Ho CCK, Jackson A, et al. Quantifying antivascular effects of monoclonal antibodies to vascular endothelial growth factor: insights from imaging. Clin Cancer Res. 2009 Nov 1;15(21):6674-82.

76. Rajagopalan S, Mohler ER, Lederman RJ, Mendelsohn FO, Saucedo JF, Goldman CK, et al. Regional angiogenesis with vascular endothelial growth factor in peripheral arterial disease: a phase II randomized, double-blind, controlled study of adenoviral delivery of vascular endothelial growth factor 121 in patients with disabling intermittent cl. Circulation. 2003 Oct 21;108(16):1933-8.

77. Watts GF, Ooi EMM, Chan DC. Demystifying the management of hypertriglyceridaemia. Nat Rev Cardiol. 2013 Nov;10(11):648-61.

78. Ganaha F, Kao EY, Wong H, Elkins CJ, Lee J, Modanlou S, et al. Stent-based controlled release of intravascular angiostatin to limit plaque progression and in-stent restenosis. J Vasc Interv Radiol. 2004 Jun;15(6):601-8.

79. Stefanadis C, Toutouzas K, Stefanadi E, Lazaris A, Patsouris E, Kipshidze N. Inhibition of plaque neovascularization and intimal hyperplasia by specific targeting vascular endothelial growth factor with bevacizumab-eluting stent: an experimental study. Atherosclerosis. 2007 Dec;195(2):269-76.

80. Haver VG, Slart RHJA, Zeebregts CJ, Peppelenbosch MP, Tio RA. Rupture of vulnerable atherosclerotic plaques: microRNAs conducting the orchestra? Trends Cardiovasc Med. Elsevier Inc.; 2010 Feb;20(2):6571.

81. Kawabe J-I, Hasebe N. Role of the vasa vasorum and vascular resident stem cells in atherosclerosis. Biomed Res Int. 2014;2014(Table 1):701571.

82. Bhardwaj S, Roy H, Heikura T, Ylä-Herttuala S. VEGF-A, VEGF-D and VEGF-D(DeltaNDeltaC) induced intimal hyperplasia in carotid arteries. Eur J Clin Invest. 2005 Nov;35(11):669-76. 
83. Bhardwaj S, Roy H, Kärpänen $\mathrm{T}, \mathrm{He} \mathrm{Y}, \mathrm{Hi} \mathrm{Y}$, Jauhiainen S, et al. Periadventitial angiopoietin-1 gene transfer induces angiogenesis in rabbit carotid arteries. Gene Ther. 2005 Mar;12(5):388-94.

84. Bhardwaj S, Roy H, Babu M, Shibuya M, Yla-Herttuala S. Adventitial gene transfer of VEGFR-2 specific VEGF-E chimera induces MCP-1 expression in vascular smooth muscle cells and enhances neointimal formation. Atherosclerosis. Elsevier Ireland Ltd; 2011 Nov;219(1):84-91.

85. Khurana R, Zhuang Z, Bhardwaj S, Murakami M, De Muinck E, Yla-Herttuala S, et al. Angiogenesisdependent and independent phases of intimal hyperplasia. Circulation. 2004 Oct 19;110(16):2436-43.

86. Al-Mashhadi RH, Sørensen CB, Kragh PM, Christoffersen C, Mortensen MB, Tolbod LP, et al. Familial hypercholesterolemia and atherosclerosis in cloned minipigs created by DNA transposition of a human PCSK9 gain-of-function mutant. Sci Transl Med. 2013 Jan 2;5(166):166ra1.

87. Rissanen TT, Ylä-Herttuala S. Current status of cardiovascular gene therapy. Mol Ther. 2007 Jul;15(7):1233-47.

88. Rissanen TT, Korpisalo P, Markkanen JE, Liimatainen T, Ordén M-R, Kholová I, et al. Blood flow remodels growing vasculature during vascular endothelial growth factor gene therapy and determines between capillary arterialization and sprouting angiogenesis. Circulation. 2005 Dec 20;112(25):3937-46.

89. Chipumuro E, Marco E, Christensen CL, Kwiatkowski N, Zhang T, Hatheway CM, et al. CDK7 inhibition suppresses super-enhancer-linked oncogenic transcription in MYCN-driven cancer. Cell. 2014 Nov 20;159(5):1126-39.

90. Wang X, Hayashi S, Umezaki M, Yamamoto T, Kageyama-Yahara N, Kondo T, et al. Shikonin, a constituent of Lithospermum erythrorhizon exhibits anti-allergic effects by suppressing orphan nuclear receptor $\mathrm{Nr} 4 \mathrm{a}$ family gene expression as a new prototype of calcineurin inhibitors in mast cells. Chem Biol Interact. 2014 Oct 29;224C:117-27.

91. Damrauer SM, Fisher MD, Wada H, Siracuse JJ, da Silva CG, Moon K, et al. A20 inhibits post-angioplasty restenosis by blocking macrophage trafficking and decreasing adventitial neovascularization. Atherosclerosis. Elsevier Ireland Ltd; 2010 Aug;211(2):404-8.

92. Hutter R, Speidl WS, Valdiviezo C, Sauter B, Corti R, Fuster V, et al. Macrophages transmit potent proangiogenic effects of oxLDL in vitro and in vivo involving HIF-1 $\alpha$ activation: a novel aspect of angiogenesis in atherosclerosis. J Cardiovasc Transl Res. 2013 Aug;6(4):558-69.

93. Wang $\mathrm{Y}$, Zhou $\mathrm{Y}, \mathrm{He} \mathrm{L}$, Hong $\mathrm{K}$, Su H, Wu Y, et al. Gene delivery of soluble vascular endothelial growth factor receptor-1 (sFlt-1) inhibits intra-plaque angiogenesis and suppresses development of atherosclerotic plaque. Clin Exp Med. 2011 Jun;11(2):113-21.

94. Lee SC, Carr CL, Davidson BP, Ellegala D, Xie A, Ammi A, et al. Temporal characterization of the functional density of the vasa vasorum by contrast-enhanced ultrasonography maximum intensity projection imaging. JACC Cardiovasc Imaging. Elsevier Inc.; 2010 Dec;3(12):1265-72.

95. Herrmann J, Best PJ, Ritman EL, Holmes DR, Lerman LO, Lerman A. Chronic endothelin receptor antagonism prevents coronary vasa vasorum neovascularization in experimental hypercholesterolemia. J Am Coll Cardiol. 2002 May 1;39(9):1555-61.

96. Gössl M, Herrmann J, Tang H, Versari D, Galili O, Mannheim D, et al. Prevention of vasa vasorum neovascularization attenuates early neointima formation in experimental hypercholesterolemia. Basic Res Cardiol. 2009 Nov;104(6):695-706.

97. Kwon HM, Sangiorgi G, Ritman EL, McKenna C, Holmes DR, Schwartz RS, et al. Enhanced coronary vasa vasorum neovascularization in experimental hypercholesterolemia. J Clin Invest. 1998 Apr 15;101(8):1551-6.

98. Aoki T, Rodriguez-Porcel M, Matsuo Y, Cassar A, Kwon T-G, Franchi F, et al. Evaluation of coronary adventitial vasa vasorum using 3D optical coherence tomography--animal and human studies. 
Atherosclerosis. Elsevier Ltd; 2015 Mar;239(1):203-8.

99. Xu X, Mao W, Chai Y, Dai J, Chen Q, Wang L, et al. Angiogenesis Inhibitor, Endostar, Prevents Vasa Vasorum Neovascularization in a Swine Atherosclerosis Model. J Atheroscler Thromb. 2015 Oct 1;22(10):1100-12.

100. Pels K, Labinaz M, Hoffert C, O’Brien ER. Adventitial angiogenesis early after coronary angioplasty : correlation with arterial remodeling. Arterioscler Thromb Vasc Biol. 1999 Feb;19(2):229-38.

101. Pels K, Deiner C, Coupland SE, Noutsias M, Sutter AP, Schultheiss HP, et al. Effect of adventitial VEGF(165) gene transfer on vascular thickening after coronary artery balloon injury. Cardiovasc Res. 2003 Dec $1 ; 60(3): 664-72$.

102. Cheema AN, Hong T, Nili N, Segev A, Moffat JG, Lipson KE, et al. Adventitial microvessel formation after coronary stenting and the effects of SU11218, a tyrosine kinase inhibitor. J Am Coll Cardiol. 2006 Mar 7;47(5):1067-75. 
Chapter 7 


\title{
Chapter 8
}

\author{
Summary
}

Samenvatting

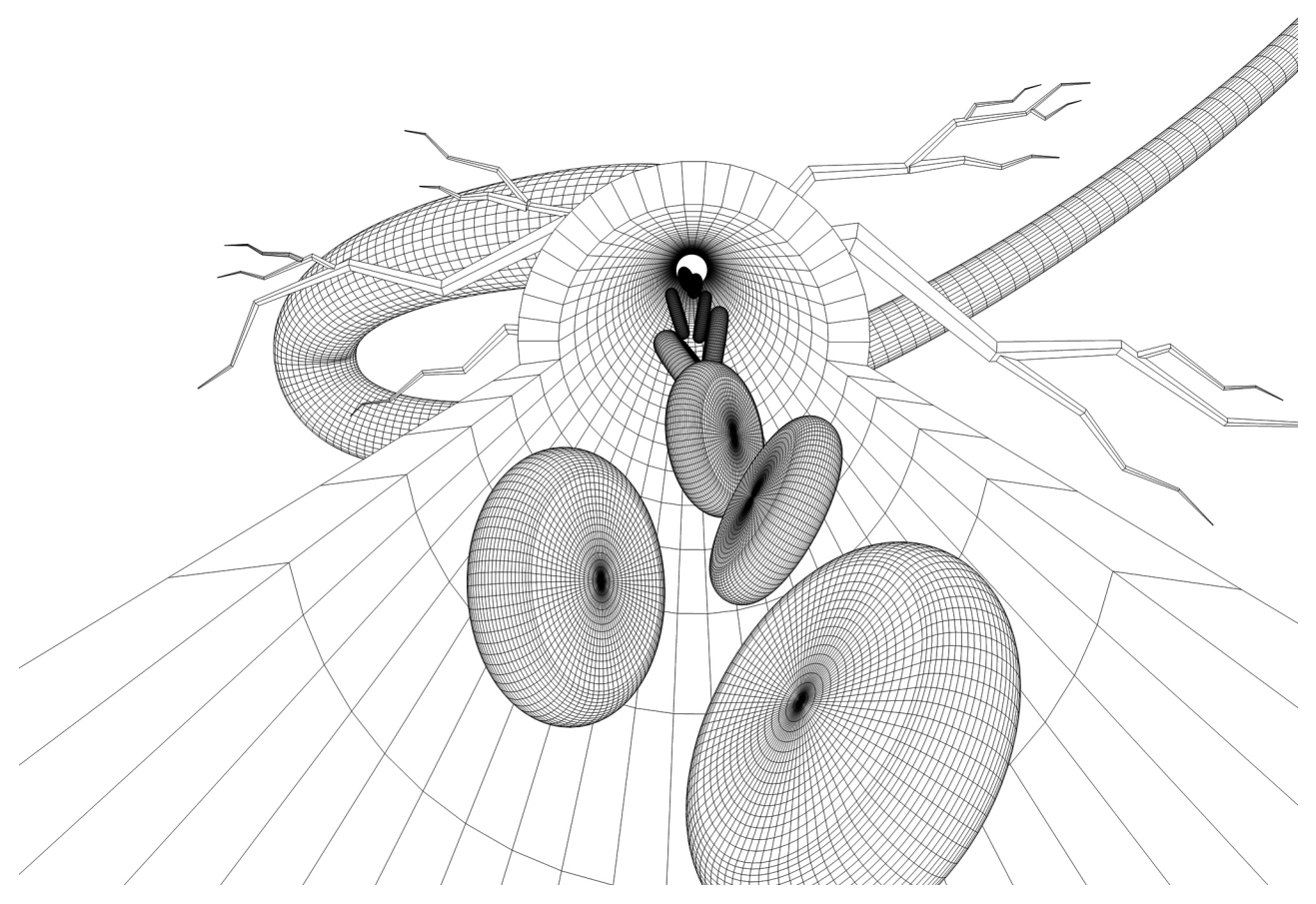




\section{SUMMARY}

Cardiovascular disease, such as myocardial infraction or stroke, still remains the major cause of death in the Western world. Rupture of an atherosclerotic plaque has been identified as the underlying cause of these life threatening conditions. Rupture susceptibility in humans has been associated amongst others with plaque hypoxia (lack of oxygen) and plaque angiogenesis (microvessel growth). Thus, the overall aim of the studies presented in this thesis was to explore a causal role for plaque hypoxia and angiogenesis in experimental murine atherosclerosis.

Prior studies showed that hypoxia correlated mainly with macrophages in human atherosclerotic plaques. Here we were able to show that also much smaller murine plaques are hypoxic, from disease initiation onwards (chapter 2). Furthermore, we hypothesized that restoring plaque oxygenation would stabilize atherosclerosis (chapter 2). We were able to show that restoring plaque oxygen levels, thus reversing hypoxia, by hyperoxic carbogen gas $\left(95 \% \mathrm{O}_{2}, 5 \% \mathrm{CO}_{2}\right)$ treatment of atherosclerosis prone mice, is indeed beneficial in experimental atherosclerosis. Reoxygenation hampered necrotic core formation by increased phagocytic removal of apoptotic cells.

An important consequence of hypoxia is the induction of angiogenesis through oxygendependent enzymes (PHD) and transcription factors (HIF). Plaque angiogenesis has been associated with intra-plaque hemorrhage and plaque rupture in humans. In murine plaques, angiogenesis is scarce, despite the presence of hypoxia and occasional intraplaque hemorrhage. Most murine studies have thus resorted to use adventitial angiogenesis as a surrogate. Although the presence of intra-plaque angiogenesis would be needed for murine plaques to more closely resemble human plaques, and processes taking place therein. Therefore, we hypothesized that increasing the molecular hypoxia signaling in macrophages would increase plaque angiogenesis and as a consequence increase plaque size and instability (chapter 3). A macrophage-specific knock out model of the dominant hypoxia sensing protein prolyl hydroxylase domain protein (PHD) 2 was used in a model of experimental atherosclerosis. PHD2 KO is known to lead to increased protein levels of HIF1 $\alpha$, which in turn triggers angiogenesis. Despite a lack of plaque angiogenesis and no changes in adventitial angiogenesis, plaque size increased in PHD2 deficient mice. The larger plaques were attributed to elevated plaque collagen leading to increased plaque stability.

The absence of vessel surrounding stabilizing cells (pericytes) is an important determinant of microvessel permeability. This is regulated by anchoring of "platelet derived growth factor B" (PDGF-B) in the extracellular matrix which facilitates pericyte recruitment towards microvessels. Deficiency of PDGF's retention motif prevents PDGF-B from anchoring and consequently the migration of pericytes thereby increasing microvessel permeability. It was hypothesized that decreased pericyte recruitment due to retention motif deficiency (PDGF-B ${ }^{\text {ret/ret}}$ ) would lead to increased hyper-permeability and finally exacerbation of plaque development (chapter 4 ). To investigate this hypothesis PDGF$B^{\text {ret/ret }}$ mice were crossed with hypercholesterolemic mice. In contrast to the hypothesis adventitial microvessel density and quality were unchanged in PDGF-B ${ }^{\text {ret/ret }}$ mice, despite an increased plaque area. PDGF-B ${ }^{\text {ret/ret }}$ resulted in a pro-inflammatory phenotype of circulating leukocytes, which was not resembled in the plaques as the percentage of 
macrophages remained similar. The increase in plaque area could be explained by an increase in collagen deposition in the plaques, independent of smooth muscle cell density. As previously mentioned, microvessel quality has been associated with plaque rupture in humans and improving microvessel quality and functionality (microvessel normalization), might thus reduce plaque burden. Microvessel normalization is established for oncological applications. As microvessel appearance found in ruptured human atherosclerotic plaques widely phenocopies leaky tumor vessels, this strategy may be feasible for atherosclerosis. In tumors, as well as in atherosclerotic plaques, high levels of the vascular growth factor Angiopoietin-2 (Ang-2) were identified associated with leaky microvessels. Moreover, in an experimental sepsis model anti-Ang-2 therapy normalized the leaky vessels and improved disease outcome. It is conceivable that microvessel normalization via Ang-2 depletion is also applicable for the treatment of atherosclerosis. Thus, it was hypothesized that depletion of Ang-2 would normalize existing plaque vessels and decrease experimental atherosclerosis (chapter 5). However, Ang-2 depletion had no effect on microvessels and existing atherosclerotic plaques. Instead Ang-2 depletion delayed plaque initiation, presumably via decreasing plasma triglyceride levels.

The poor microvessel structure and ensuing microvascular leakage is thought to cause intra-plaque hemorrhage. Microvascular (hyper-) permeability has been linked to an imbalance of vascular growth factors, including members of the vascular endothelial growth factor (VEGF) family in atherosclerosis: VEGF-A, D and $F$. The varying potential of the different VEGFs to evoke vascular permeabilitycompared to vaso-active factors such as histamine is not known. In chapter 6 we compared the potential of the different VEGFs (VEGF-A, D and F) and histamine to instigate hyper-permeability on a functional level. In addition, the effects on the transcriptome of endothelial cells after stimulation with VEGF$A$ and histamine was analyzed using RNA sequencing. Interetsingly, almost $50 \%$ of genes regulated by VEGF-A, are also regulated by histamine. The obtained molecular insights may be used to prevent edema formation as a side effect of VEGF gene therapy.

The experiments described in this thesis led us to conclude, that manipulating plaque hypoxia and angiogenesis in experimental atherosclerosis to understand the underlying mechanisms and causal relation to atherogenesis is challenging. Although plaque hypoxia was reversible and causally related to atherogenesis, more research is needed to translate this systemic treatment to a molecular therapy. Angiogenesis in atherosclerosis is difficult to study in mouse models, as murine plaques lack microvessels, adventitial microvessels are a poor surrogate of intra-plaque vessels and our experiments increasing microvessel numbers or altering microvessel quality have failed. Interference with the vascular growth factor Ang-2 in experimental atherosclerosis showed that treatment with anti-Ang-2 therapy is likely not harmful from a cardiovascular point of view. In comparison, manipulation of the vascular growth factor PDGF-B is rather unfavorable for atherosclerosis treatment. Moreover, safety of using VEGFs for pro-angiogenic therapy could be improved by considering the identified downstream pathways inducing permeability in endothelial cells.

In conclusion interfering with hypoxia in atherosclerosis shows promising results, however manipulation of the molecular hypoxia mechanism bares potential dangers. Angiogenesis seems detrimental for human atherosclerosis but a causal relation remains to be established for example in (animal) mouse models, however, these have been proven 
Chapter 8

unsuitable thus far. VEGF and histamine share common molecular pathways in endothelial cells which may help to improve the safety of angiogenic therapy in the future. 


\section{SAMENVATTING}

De gevolgen van aderverkalking of atherosclerose, zoals een hartaanval of een beroerte, zijn de belangrijkste doodsoorzaak in de huidige welvaartssamenleving. Een plaque ruptuur, het scheuren van fibrotische cap van een atherosclerotische plaque wordt gezien als de onderliggende oorzaak. Het risico op een plaque ruptuur wordt in verband gebracht met o.a. een gebrek aan zuurstof (hypoxie) en de vorming van microvaten (angiogenese) in de plaque. Het overkoepelende doel van de studies, die beschreven worden in dit proefschrift, was om een causale rol van plaque hypoxie en angiogenese in diermodellen van aderverkalking te onderzoeken.

Er was reeds aangetoond dat de aanwezigheid van hypoxie in humane atherosclerotische plaques vooral correleerde met macrofagen. In hoofdstuk 2 werd aangetoond dat ondanks het kleinere oppervlakte van plaques in muizen, de plaques ook hypoxisch zijn direct vanaf de vorming van een nieuwe plaque. Vervolgens was de hypothese geformuleerd, dat herstel van het zuurstof gehalte in een plaque (reoxygenatie) atherosclerose zou verminderen. We hebben aangetoond dat herstel van plaque hypoxie door een behandeling met hyperoxisch carbogeen gas $\left(95 \% \mathrm{O}_{2}, 5 \% \mathrm{CO}_{2}\right)$, inderdaad atherosclerose in een muismodel vermindert. Reoxygenatie voorkwam groei van de necrotische kern door een verbeterde opruiming van dode cellen door fagocyten.

In het algemeen stimuleert hypoxie, via signalering door zuurstof-afhankelijke enzymen en transcriptie factoren, angiogenese om het zuurstofgebrek op te heffen. Angiogenesis in humane plaques is gecorreleerd aan intraplaque bloedingen en plaque ruptuur. In muizen plaques is angiogenese schaars, ondanks de aanwezigheid van hypoxie en intraplaque bloedingen. In de meerderheid van atherosclerose studies met muizen wordt dan ook angiogenese in de adventitia als surrogaat gebruikt. Echter, de aanwezigheid van microvaten in de muizenplaque zou het muis model sterk verbeteren door een grotere vergelijkbaarheid met de morfologie van een humane plaque. Hierdoor zouden ook de verantwoordelijke moleculaire processen beter te bestuderen zijn. We hebben dan ook de hypothese geformuleerd dat plaque angiogenese - en dus ook plaque groei en destabiliteit- versterkt worden door de zuurstofafhankelijke signaleringscascades te stimuleren (hoofdstuk 3). Een macrofaag-specifieke knock-out (KO) van de zuurstof sensor "prolyl hydroxylase domain protein" (PHD) 2 werd voor dit doel gebruikt in een muismodel van atherosclerose. Er werd reeds aangetoond dat PHD2 KO het eiwitniveau van HIF1 $\alpha$ verhoogt, wat vervolgens angiogenese stimuleert. Echter, PHD2 KO in macrofagen in muizen plaques resulteerde niet in verhoogde microvaatdichtheid in plaques of adventitia, maar wel tot een toename in plaque oppervlakte. Een toename in plaque collageen verklaarde de grotere plaques, maar verbeterde desondanks wel de plaque stabiliteit.

Microvaatdichtheid is gecorreleerd met het risico op humane plaque ruptuur. Meer recent werd ook de gebrekkige kwaliteit van microvaten en de resulterende hyperpermeabiliteit gerelateerd aan klinische symptomen van hart- en vaatziekten. De afwezigheid van verstevigende cellen (pericyten) rondom microvaten is een belangrijke determinant van microvasculaire permeabiliteit. Dit wordt gereguleerd door de verankering van "platelet derived growth factor B" (PDGF-B) in de extracellulaire matrix, wat leidt tot rekrutering van pericyten naar microvaten. Deficiëntie van het retentiemotief voorkomt de verankering van PDGF-B, de migratie van periycten en verhoogde microvasculaire 
permeabiliteit. Er werd dan ook de hypothese geformuleerd, dat verlaagde pericyte rekrutering door deficiëntie van het retentiemotief (PDGF-B ${ }^{\text {ret/ret}}$ ) zou leiden tot microvasculaire hyperpermeabiliteit en vervolgens plaque ontwikkeling zou versterken (hoofdstuk 4). PDGF-B ${ }^{\text {ret/ret }}$ muizen werden gekruist met hypercholesterolemische muizen om deze hypothese te bestuderen. In tegenstelling tot de hypothese waren adventitiale microvaatdichtheid en kwaliteit onveranderd in PDGF-B ${ }^{\text {ret/ret }}$ muizen, maar desondanks was het plaque oppervlakte toegenomen. PDGF-B ${ }^{\text {ret/ret }}$ resulteerde in een proinflammatoir fenotype van circulerende leukocyten, desondanks leidde dit tot gelijke macrofaagdichthied in de plaque. Het toegenomen plaque oppervlakte werd verklaard door verhoogde collageen depositie in de plaque onafhankelijk van gladde spiercel dichtheid in de plaques.

Zoals genoemd, is microvasculaire kwaliteit gecorreleerd met humane plaque ruptuur. De verbetering van microvasculaire kwaliteit en barrière functie, zogenaamde normalisatie, kan mogelijk plaque ruptuur voorkomen. Het principe van microvasculaire normalisatie wordt al toegepast in oncologisch onderzoek. Aangezien de morfologie van tumor microvaten overeenkomt met plaque microvaten, lijkt deze tactiek kansrijk. In tumoren en plaques is de vasculaire groeifactor "Angiopoietin-2" (Ang-2) gecorreleerd met hyperpermeabele microvasculatuur. Bovendien, normaliseerde anti-Ang-2 therapie microvasculaire hyperpermeabilieit ten gevolge van sepsis in een experimenteel diermodel. Het is dus aannemelijk dat microvasculaire normalisatie door Ang-2 depletie ook toepasbaar is voor de benadeling van atherosclerose. De hypothese werd geformuleerd dat depletie van Ang-2 bestaande plaque microvaten normaliseert en de ontwikkeling van atherosclerose in een diermodel vertraagd (hoofdstuk 5). Echter in tegenstelling tot de hypothese, had Ang-2 depletie geen effect op plaque of adventitiale microvaten of bestaande plaque grootte en fenotype. Ang-2 depletie vertraagde enkel plaque initiatie, waarschijnlijk door verlaging van plasma triglycerides.

Een gebrekkige microvasculaire structuur en de daaruit volgende hyperpermeabliteit wordt gezien als de oorzaak van intra-plaque bloedingen. Microvasculaire (hyper-) permeabiliteit wordt veroorzaakt door een disbalans van vasculair groeifactoren, zoals de "vascular endothelial growth factor" (VEGF) familie: VEGF-A, D and F. Het relatieve potentieel van deze factoren om vasculair permeabiliteit ter veroorzaken in vergelijking met vasoactieve factoren zoals histamine is nog onbekend. In hoofdstuk 6 vergelijken we de transcriptionele respons en de resulterende permeabiliteit van de verschillende VEGF$A, D$ and $F$ onderling en met histamine door middel van RNA sequencing, multifoton microscopie en in vitro experimenten. Ongeveer $50 \%$ van de VEGF-A responsieve genen worden ook door histamine gereguleerd. De verkregen kennis over de moleculaire respons op VEGF kan toegepast worden om oedema vorming, een bijwerking van VEGF gen therapie, te voorkomen.

De resultaten in dit proefschrift leidden tot de conclusie dat interventies in plaque hypoxie en angiogenese in de muis om onderliggende mechanismen en een causale rol met atherogenese vast te stelen niet altijd het boogde resultaat bewerkstelligden. Ondanks de waargenomen reoxygenatie van plaque hypoxie en een causale relatie met plaque stabilisering, is meer onderzoek nodig om deze systemische behandeling te vertalen naar een moleculaire therapie. De rol van angiogenese in atherosclerose is bijna onmogelijk te bestuderen in muis modellen, omdat muizen plaques geen microvaten bevatten, en adventitiale microvaten een slecht surrogaat zijn van intraplaque vaten. Daarbij zijn onze 
interventies om een geschikt model te creëren door microvasculaire kwantiteit en kwaliteit te manipuleren, helaas niet geslaagd. Depletie van Ang-2 in muismodellen van atherosclerose leidde niet tot schadelijke neveneffecten of cardiovasculaire complicaties, terwijl interventies met de vasculaire groeifactor PDGF-B plaque groei en destabiliteit lijken te stimuleren. Verder kan de veiligheid van pro-angiogene therapie door VEGF stimulering of toediening verbeterd worden door de onderliggende moleculaire mechanismen, verantwoordelijk voor endotheliale permeabiliteit, verder te bestuderen.

In conclusie, manipulatie van hypoxie in atherosclerose levert veelbelovende resultaten, maar ingrijpen in de moleculaire hypoxie signaleringscascade vertoond mogelijk risico's. Angiogenesis lijkt nadelig te zijn voor humane atherosclerose maar een causale relatie moet nog worden aangetoond, bijvoorbeeld door middel van (dier) muis modellen, echter zijn deze tot nu toe ongeschikt hiervoor. VEGF en histamine werken deels via gelijke moleculaire signalering in endotheelcellen, deze inzicht zal in de toekomst mogelijk helpen om angiogene therapie veiliger te maken. 
Chapter 8 


\title{
Chapter 9
}

\author{
Valorization
}

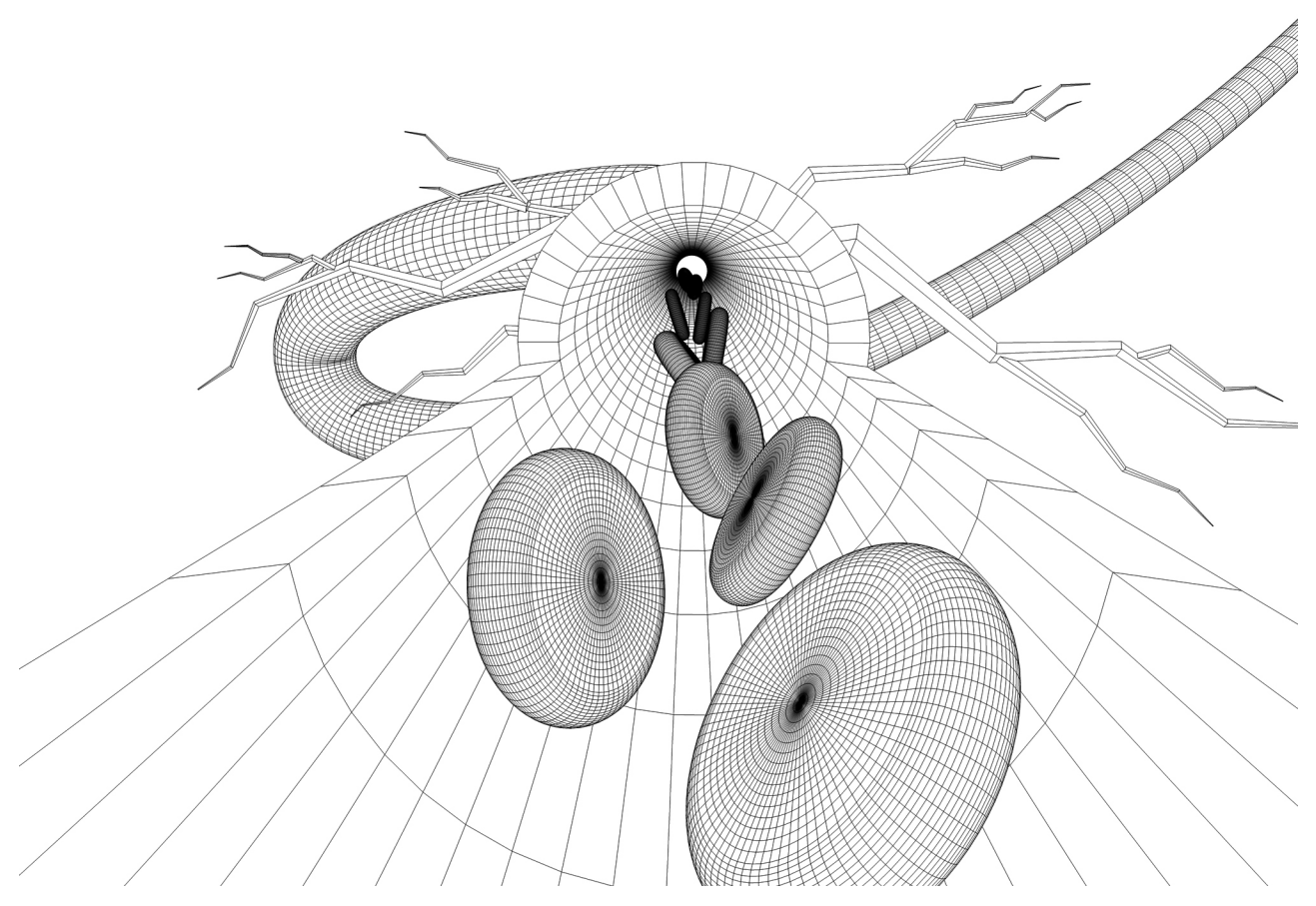




\section{Atherosclerosis: a socio-economic burden}

Valorization describes "the process of creating value from knowledge" in order to make knowledge suitable and/or available for social and/or economic use. In the opinion of the author the valorization section is of particular importance since it is the aim of the European Union (EU), in which the work was conducted (the Netherlands and Finland), to create a knowledge-based economy (1). The work presented in this thesis addresses atherosclerosis which is the underlying cause of most cardiovascular diseases and represents a major socio-economic burden. Cardiovascular diseases, such as myocardial infraction of stroke, annually account for $40 \%$ (1.9 million) of all deaths in the EU (2). Furthermore, as cardiovascular diseases are often not instantly lethal, patients may require life-long treatment and suffer from severe disabilities. In addition to the affliction of the patient, cardiovascular disease also has an impact on people in the patient's close environment. Society as a whole is affected by cardiovascular disease as the related health care costs add up to annually $196 €$ billion in the EU (2).

Public funds are dedicated to two major themes: First, to the prevention of atherosclerosis and second, to its treatment. Reducing well established classical risk factors such as obesity, smoking, alcohol consumption, high blood pressure and high plasma LDL cholesterol helps to prevent atherosclerosis. However, not all cases can and will be prevented as unwillingness to adapt lifestyle, poor therapy compliance and genetic disorders such as familial hypercholesterolemia remain problematic. Therefore, exploring treatment options for existing and symptomatic atherosclerosis is a necessity. In 1987, statins, a drug primarily designed for cholesterol lowering, became commercially available and is since than the only frequently used treatment (3). Statins cannot cure cardiovascular disease as treatment reduces overall cardiovascular mortality with $12 \%$ and lowers the risk for major coronary and cerebrovascular events by $30 \%$ and $19 \%$ repspectively (4). In addition long term statins use may cause side effects such as myopathy (5).

Thus, the current societal burden resulting from atherosclerosis raises demand for new innovative treatment strategies. This implies that important underlying experimental concepts to open new therapeutic opportunities have to be developed and need to become publicly available.

\section{Public availability of research results}

The author's interest in making the results of the publically funded research available for society is depicted in the aim of publishing the obtained results in peer reviewed journals. This aim was accomplished in chapter $\mathbf{2}$ and chapter $\mathbf{5}$ as both are published in journals relevant for atherosclerosis research, in "Arteriosclerosis, Thrombosis, and Vascular Biology" and "Atherosclerosis" respectively. In addition to the already published chapters, chapter 3 and chapter 6 have been submitted for publication. The aim is to also publish the work presented in chapter 4, however, additional experiments are necessary to underline our preliminary findings. Publishing in peer reviewed journals does not only provide for knowledge dissemination, but also assures the scientific quality as experts in the field evaluate the submitted article prior to publication. The chosen journals are frequented by scientist, as well as medical personnel, making possible future translation of the research presented here more likely, as direct integration into the daily clinical 
practice or commercial products is not applicable at the current state. The major limitation for a rapid translation is the use of mouse models, which do provide solid indications for basic mechanisms but unfortunately leave a gap between rodent and human atherosclerosis (6).

\section{Generated novel insights with potential socio-economic value}

Clinical complications arising from atherosclerosis such as myocardial infraction or stroke are based on plaque rupture. Thus, delaying plaque formation or plaque stabilization of an atherosclerotic plaque in the performed animal experiments is defined by the author as finding with a potential value.

Chapter 2 demonstrated how oxygen treatment successfully hampered arthrosclerosis progression by reducing necrotic core formation in murine atherosclerosis. Furthermore, the underlying mechanism was partially unraveled which may represent a starting point for future therapeutic strategies. As oxygen treatment is also successful, but impractical in treating human atherosclerosis, the same underlying mechanisms found in the mouse model could be applied to humans. Mechanistically, it was found that hypoxia downregulated the efferocytosis receptor MerTK, resulting in accumulation of dead cells and necrotic core formation. After validation of the mechanism in humans, treatment aiming at upregulating MerTK receptor expression could be designed.

In chapter 3 plaque stabilization, by enhancing plaque collagen and cap thickness after PHD2 inhibition in macrophages, was shown in an animal model. However, PHD2 inhibition simultaneously increased plaque size, demonstrating the risk for potential side effects of PHD2 inhibition. PHD inhibitors are currently investigated in clinical trials for the treatment of anemia in patients with chronic kidney disease (NCT02174731). These are neither isoform-specific, nor targeted to macrophages, but since PHD2 inhibition had an effect on experimental atherosclerosis (7), investigating its effects on atherosclerosis in the ongoing clinical trials could be considered. The effect of PHD inhibition could be ambivalent, thus, either the currently tested drug may be beneficial or harmful for atherosclerosis development. To obtain an isolated effect, isoform-specific, macrophagetargeted PHD inhibitors would need to be developed.

In chapter $\mathbf{5}$ systemic Ang-2 inhibition was observed to decelerate plaque initiation likely via reducing plasma triglyceride levels. Reducing plasma triglycerides levels could also be beneficial for humans as these have a proven pro-atherogenic function. The anti-Ang-2 antibody used in chapter 2 is currently investigated in a clinical trial to treat unresectable stage III or stage IV melanoma (NCT02141542). Thus, the effect on triglyceride lowering in the animal model could be validated in the humans receiving the antibody. The frequent use of an antibody for reducing triglycerides is impractical for clinical practice as it is injected. General Ang-2 inhibition via small molecule inhibitors could overcome the practical concerns. However, Ang-2 is an important factor in angiogenesis and long term Ang-2 inhibition for triglyceride reduction may thus have unpredictable side effects. Besides, the presented results indicate a safe use of the Ang-2 antibody in cancer treatment with regard to atherosclerosis, but note that the data was generated based on an animal model.

Chapter 6 shows the common regulation of several genes associated with endothelial hyper-permeability by VEGF-A and histamine. VEGF-A gene therapy bares the potential to treat ischemic diseases such as peripheral artery disease by the stimulation of 
angiogenesis and subsequent revascularization of ischemic areas. A known side effect of VEGF-A gene therapy is the formation of edema as a consequence of its potential to evoke hyper-permeability. Our obtained results, the partial identification of the underlying pathways of VEGF-A and histamine induced hyper-permeability, might thus help to prevent edema formation in VEGF-A gene therapy thereby increasing the safety of this promising therapeutic tool. Amongst others MYCN and NR4A1 could be identified in the permeability induction cascade and their pharmacological inhibition by for instance existing CDK7 inhibitors or calcineurin inhibitors, respectively could be considered. However, additional experimental work is needed to verify the suggested approach for future clinical translation.

In conclusion, our findings are not applicable for direct translation into the clincical practice, but they provide a scientific basis for future developments in atherosclerosis research, and potentially even the development of new therapies. Furthermore, our results suggest to explore additional applications for existing therapeutic agents in atherosclerosis. 


\section{References}

1. European Council. Lisbon European Council Conclusions [Internet]. 2000 [cited 2016 Jan 15]. Available from: http://www.consilium.europa.eu/en/workarea/downloadAsset.aspx?id=40802198157

2. Nichols M, Townsend N, Luengo-Fernandez R, Leal J, Gray A, Scarborough P RM. European Cardiovascular Disease Statistics 2012. 2012.

3. Endo A. A historical perspective on the discovery of statins. Proc Japan Acad Ser B. 2010;86(5):484-93.

4. Brugts JJ, Yetgin T, Hoeks SE, Gotto AM, Shepherd J, Westendorp RGJ, et al. The benefits of statins in people without established cardiovascular disease but with cardiovascular risk factors: meta-analysis of randomised controlled trials. BMJ. 2009 Jun 30;338(jun30 1):b2376.

5. Kapur NK, Musunuru K. Clinical efficacy and safety of statins in managing cardiovascular risk. Vasc Health Risk Manag. 2008;4(2):341-53.

6. Libby P. Murine "Model” Monotheism: Figure. Circ Res. 2015 Nov 6;117(11):921-5.

7. Rahtu-Korpela L, Määttä J, Dimova EY, Hörkkö S, Gylling H, Walkinshaw G, et al. Hypoxia-Inducible Factor-Prolyl 4-Hydroxylase-2 Inhibition Protects Against Development of Atherosclerosis. Arterioscler Thromb Vasc Biol. 2016 Feb 4;ATVBAHA.115.307136. 
Chapter 9 


\section{Appendices}

\section{List of abbreviations}

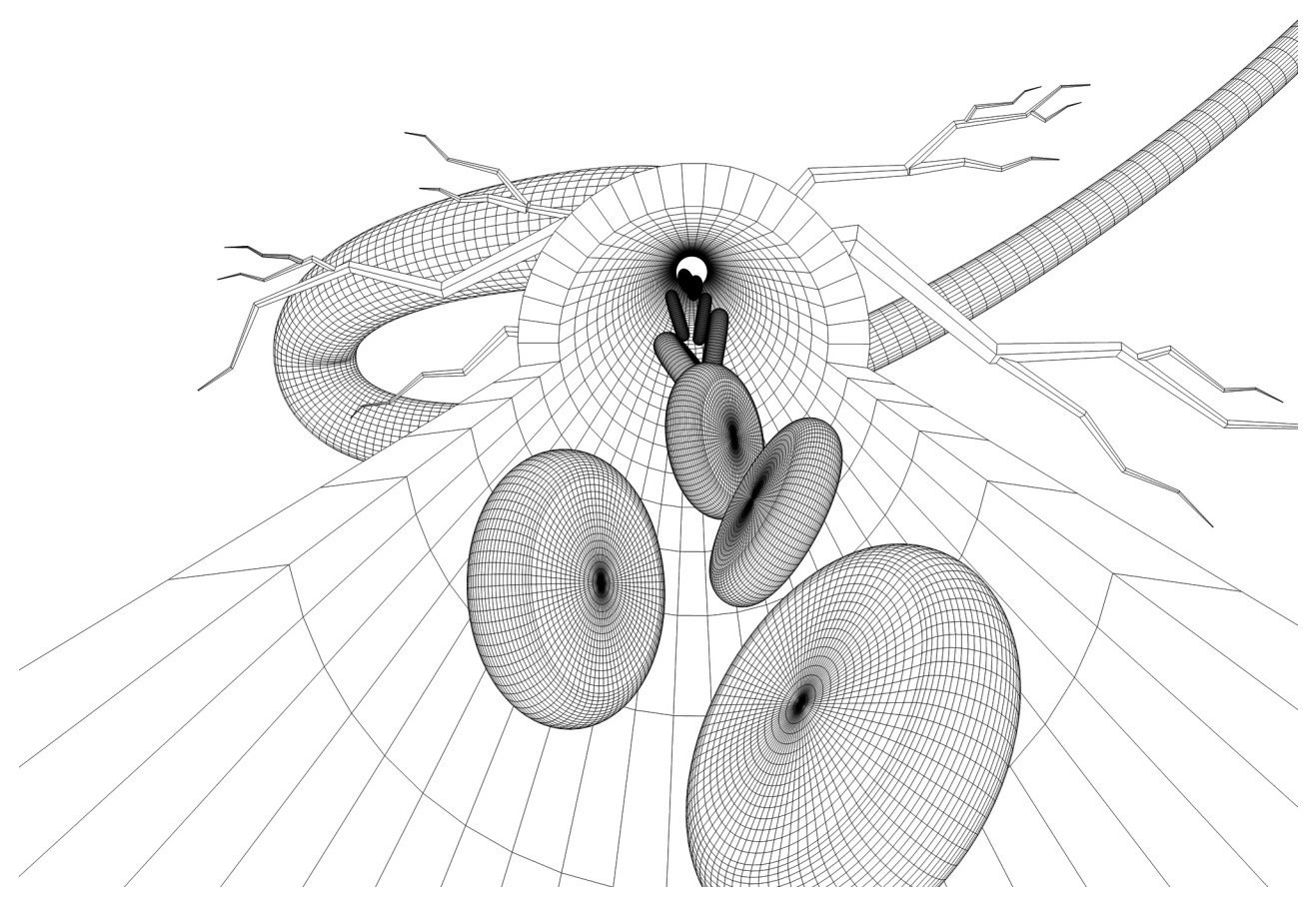


8OH-dG

ADAM

AJ

Ang

ANO1

Apo

AQP1

ATF4

ATP

ATP2B1

BMDM

CD

CDC

CGNL1

cKO

CLDN

Coll

Cre

CXADR

$\mathrm{CXCL}$

$D A B$

$D C$

DMEM

EC

ECM

EDTA

EF

ELISA

ER

FACS

FCS

FDG

FGF

$\mathrm{fl}$

FS

GLUT

$\mathrm{GO}$ 8-hydroxy-2'deoxy-guanosine

A desintegrin metallo protease

Adherens junction

Angiopoietin

$\mathrm{Ca}^{2+}$-activated chloride channel anoctamin 1

Apolipoprotein

Aquaporin 1

Activation transcription factor 4

Adenosine triphosphate

Plasma membrane calcium transporting ATPase 1

Bone marrow derived macrophage

Cluster of differentiation

Conventional dendritic cell

Cingulin-like 1

Conditional knock out

Claudin

Collagen

Cre recombinase

Coxsackie virus and adenovirus receptor

Chemokine (C-X-C motif) ligand

Diaminobenzidine

Dendritic cell

Dulbecco's modified Eagle's medium

Endothelial cell

extracellular matrix

Ethylenediaminetetraacetic acid

Ejection fraction

Enzyme-linked immunosorbent assay

Endoplasmatic reticulum

Fluorescence-activated cell sorting

Fetal calf serum

Fluorine-labeled 2-deoxy-D-glucose

Fibroblast growth factor

Flox

Fractional shortening

Glucose transporter

Gene ontology 


\begin{tabular}{|c|c|}
\hline GPCR & G-protein-coupled receptor \\
\hline HAEC & Human aortic endothelial cells \\
\hline $\mathrm{HE}$ & Hematoxylin and eosin \\
\hline HGF & Hepatocyte growth factor \\
\hline HIF & Hypoxia inducible factor \\
\hline$H R$ & Histamine receptor \\
\hline HRE & Hypoxia responsible element \\
\hline HSPGs & Heparan sulfate proteoglycans \\
\hline HUVEC & Human umbilical vein endothelial cells \\
\hline i.v. & Intravenous \\
\hline IFN & Interferon \\
\hline $\lg$ & Immunoglobulin \\
\hline IKK $\beta$ & Inhibitor of kappa B kinase $\beta$ \\
\hline IL & Interleukin \\
\hline iNOS & Nitric oxide synthase \\
\hline JAM & Junction adhesion molecule \\
\hline KO & Knock out \\
\hline LCM & L929-conditioned medium \\
\hline LDL & Low density lipoprotein \\
\hline LDLr & Low density lipoprotein receptor \\
\hline LPS & Lipopolysaccharide \\
\hline LRP-1 & Low density lipoprotein receptor-related protein 1 \\
\hline LV & Left ventricle \\
\hline LysM & Lysozyme M \\
\hline MerTK & Mer tyrosine kinase domain \\
\hline MMP & Matrix metalloprotease \\
\hline MR & Mannose receptor \\
\hline MVD & Microvessel density \\
\hline MYCN & $\mathrm{N}$-myc proto-oncogene protein \\
\hline NFKB & Nuclear factor kappa beta \\
\hline NO & Nitric oxide \\
\hline Notx & Non-treated endothelial cells \\
\hline NR4A1 & Nuclear receptor subfamily 4 group A member 1 \\
\hline NRP & Neuropilin \\
\hline OCT & Optical coherence tomography \\
\hline oxLDL & Oxidized low density lipoprotein \\
\hline p.t. & Post transduction \\
\hline
\end{tabular}




\begin{tabular}{|c|c|}
\hline $\mathrm{P} 4 \mathrm{HA}$ & Collagen prolyl 4-hydroxylase alpha \\
\hline PBS & Phosphate buffered saline \\
\hline PCR & Polymerase chain reaction \\
\hline PCSK9 & Proprotein convertase subtilisin/kexin type 9 \\
\hline $\mathrm{pDC}$ & Plasmacytoid dendritic cell \\
\hline PDGF-B & Platelet derived growth factor B \\
\hline PFA & Paraformaldehyde \\
\hline PFKFB3 & 6-phosphofructo-2-kinase/fructose-2,6-biphosphatase 3 \\
\hline PHD & Prolyl hydroxylase domain protein \\
\hline $\mathrm{pVHL}$ & von Hippel-Lindau enzyme \\
\hline qPCR & Quantitative polymerase chain reaction \\
\hline RCAN1 & Regulator of calcineurin-1 \\
\hline RELN & Serine protease reelin \\
\hline RNA-seq & RNA sequencing \\
\hline ROS & Reactive oxygen species \\
\hline RPMI & Roswell park memorial institute medium \\
\hline rRNA & Ribosomal RNA \\
\hline RT & Room temperature \\
\hline s.c. & Subcutaneous \\
\hline SEM & Standard error of the mean \\
\hline SMC & Smooth muscle cells \\
\hline SNAI2 & Snail family zinc finger 2 \\
\hline STC-1 & Stanniocalcin-1 \\
\hline TEAC & Trolox equivalent antioxidant capacity \\
\hline TF & Tissue factor \\
\hline TGF- $\beta$ & tumor growth factor $-\beta$ \\
\hline Tie2 & TEK receptor tyrosine kinase 2 \\
\hline TJ & Tight junction \\
\hline TNF- $\alpha$ & Tumor Necrosis Factor $\alpha$ \\
\hline TUNEL & Terminal deoxynucleotidyl transferase dUTP nick end labeling \\
\hline UV & Ultra violet light \\
\hline VE cadherin & Vascular endothelial cadherin \\
\hline VEGF & Vascular endothelial growth factor \\
\hline VEGFR & Vascular endothelial growth factor receptor \\
\hline WB & Western Blot \\
\hline WT & Wild type \\
\hline WTD & Western type diet \\
\hline
\end{tabular}


aSMA

$\beta(2) A R$
Alpha smooth muscle actin

$\beta(2)$-adrenergic receptor 
Appendices 


\section{Appendices}

\section{Acknowledgements}

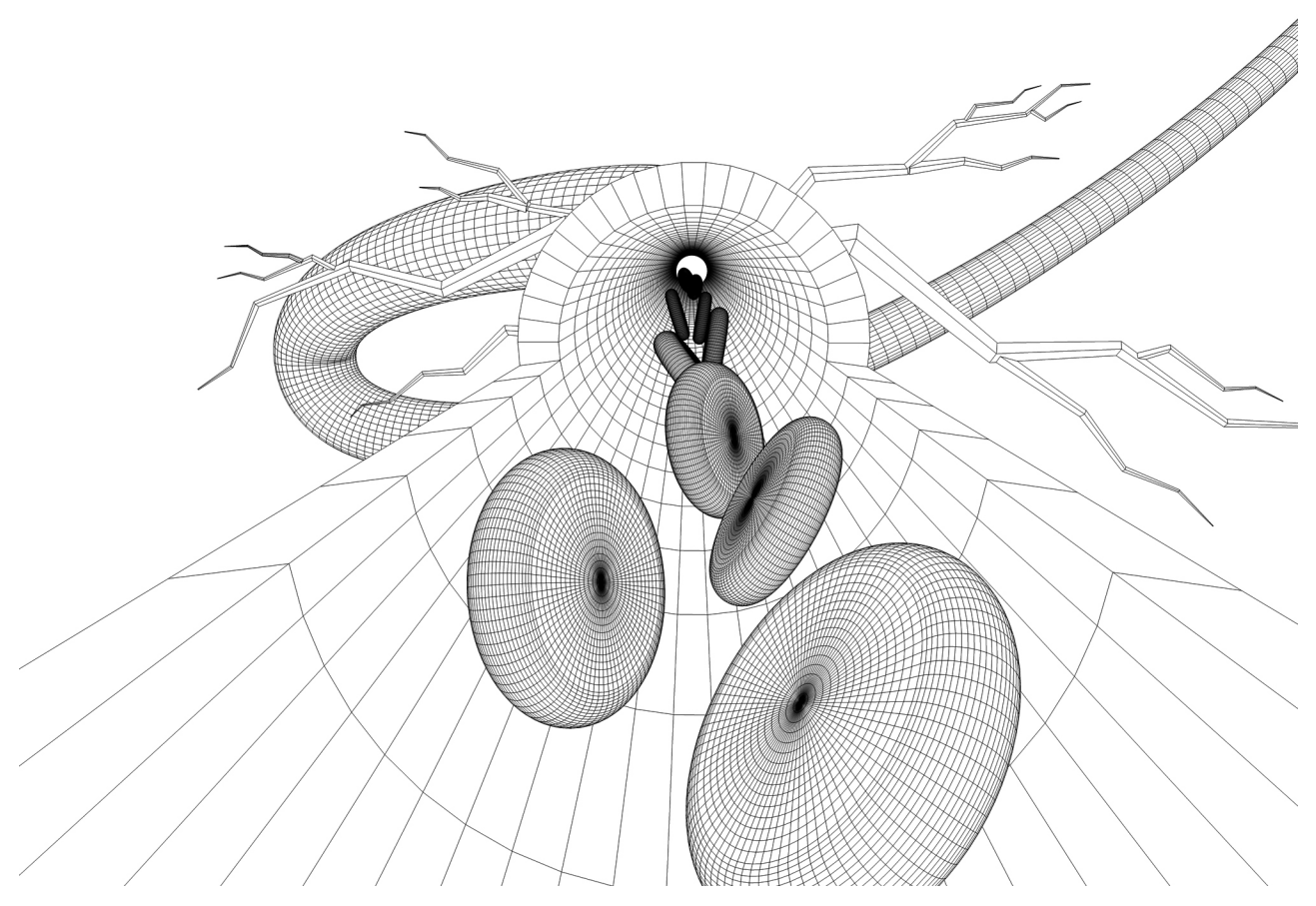


Finis coronat opus, as no thesis is complete without a Latin quote and I am in possession of a Latinum. This felt like the right moment to use it after a long time. There a lot of people that accompanied and supported me on the way to this dissertation, whom I wish to sincerely thank.

Dr. Sluimer, beste Judith, als eerste wil ik jou van harte voor je begeleiding en de kans om te mogen promoveren bedanken. Ik ben 6 jaar geleden als eerste student naar je post-doc voor jou begonnen werken en ik herinner mijn eerste dag nog als of het gisteren was. Toen kwam jij om 15.30 uur naar het lab om ter vermelden dat we nog even iets op je kamer moesten bespreken en we dan klaar waren voor die dag. Dus kwam ik met mijn jas al aan en mijn rugzak op naar jouw kamer toe. Je bent niet echt voor je pokerface bekend en naar de daaropvolgende verwoording van de eerder afleesbare emoties, zat ik heel snel weer op het lab. Dat was precies de wake-up call die ik op dat moment nodig had, hierdoor heb je mijn ambitie getriggerd en on top begon ik ook nog enorm plezier in wetenschappelijk onderzoek te vinden. In het vervolg heb je me veel over labwerk, wetenschappelijk schrijven en de wetenschap zelf geleerd en hiermee een hele nieuwe wereld voor mij geopend. Je vraagt veel en je begeleiding past niet bij iedereen, maar de ongelofelijke steun en carrière support die ik van jou heb ervaren, samen met je passie voor wetenschap is buitengewoon motiverend. De manier waarop jij met successen en mislukkingen persoonlijk of professioneel omgaat, is indrukwekkend en heeft veel aan mijn persoonlijke ontwikkeling bijgedragen. Ik heb heel veel van je geleerd ook over mezelf. Hiervoor en voor het vertrouwen dat je altijd in me had kan ik je niet genoeg danken.

Prof. dr. Daemen, beste Mat, ik wil je graag voor de wetenschappelijke en persoonlijke begeleiding in de afgelopen jaren bedanken. Ook jij hebt me als bachelor student in 2010 leren kennen en ik wist toen nog niet zo goed wat ik wilde na de bachelor. Daar was jij het niet mee eens, tenminste bleek dat uit mijn eerste sollicitatie gesprek met jou voor een student assistentschap bij Judith. Het beste laat zich dat in het Duits beschrijven met "ganz oder gar nicht". Uiteindelijk was het dus "ganz". Jouw manier van strategisch denken en het kritieke belichten van ons onderzoek liet de projecten doelgericht met het juiste oogmerk op de sterke en zwakke punten verlopen. Verder wil ik je graag ook voor je carrière advies bedanken. Na 5 jaar wetenschap stond ik weer op het punt waar ik niet precies wist wat ik wilde voor mijn toekomst, door je sturing heb ik belangrijke stappen in het voorbereiden van mijn carrière kunnen ondernemen.

Prof. dr. Biessen, beste Erik, jou wil ik ook graag bedanken voor de steun en wetenschappelijke begeleiding tijdens mijn promotie periode. Besprekingen met jou waren een bron van talloze creatieve ideeën om wetenschappelijke problemen op te lossen. Het zelfde geldt voor de gesprekken tijdens jou lab-wandelingen na de officiële uren. Bedankt voor de inspiratie.

I would like to sincerely thank the members of the assessment committee prof. dr. Tilman Hackeng, prof. dr. Robert van Oostenbrugge, prof. dr. Jürgen Bernhagen, prof. dr. Anton Jan Zonneveld, dr. Paula da Costa Martins for the time and effort you spend on the judgment and defense of my thesis. 
Without the funding provided by the CARIM PhD award the completion this thesis would not have been possible.

Prof. dr. Ylä-Herttuala, dear Seppo, two out of five chapters presented in this thesis originate from a close collaboration with you. You accommodated me for one year in your lab and I felt warmly welcome despite the Finish climate. I would like to thank you for the supervision and support you provided during my time in Koupio and beyond.

Liebe Elke, vielen Dank für deine Freundschaft und Unterstützung in den letzten 4 Jahren. Wir haben gemeinsam an fast 10 Projekten (!) gearbeitet und sind zusammen durch alle Höhen und Tiefen des Wissenschaftsalltags gegangen. Letztendlich haben wir viele Erfolge zusammen erlebt und gefeiert (Publikationen, Konferenzen, Poster Preise etc.). Genauso gut wie wir zusammen gearbeitet haben, haben wir uns auch in unserer Freizeit verstanden. Daher musste ich nicht lange überlegen wen ich als Paranymph gerne an meiner Seite haben wollte. Ich hoffe dass wir auch in Zukunft weiter so eng befreundet bleiben.

Mr. Kosta, met niemand anders op onze afdeling heb ik zo veel gelachen, foute grapjes en misgelopen pranks gemaakt. Je goede humeur is door bijna niets te verstoren. Ik heb zelden iemand leren kennen die zo positief is, dus ik ben blij je als Paranymph naast mij te hebben staan. Bedankt voor de goede tijd!

Dear Jari (aka glove-man), I would like to thank you for all I have learned from you and the awesome time we spend together working and living in Kuopio. All the countless long nights and weekends in the animal facility, operating and sacrificing together. After some drawbacks we still managed to obtain very good results and had a great time doing so. Without you my stay would have not been the same. You made me really feel at home with all the fun things we did: icehockey games, sauna nights, and many more. Kiitos for being an awesome friend and colleague.

Ik had bijzonder geluk omdat ik met zo fantastische mensen op een kamer mocht zitten: Floor, David, Dr. Lieve, Anette, Karen, Timo, Jeroen, Bart, Anke, Jasper, Margaux, Marchy, Ine, Anjana, Taghi Emiel en Jan (großer Pathologe). Promoveren doe je niet alleen, wij zijn alle in een vergelijkbare situatie en hebben elkaar altijd geholpen en gesteund. We hebben samen zo veel gelachen en gezamelijk van de sunny side of sience genoten. Ook moeilijke tijden hebben we samen overwonden. Dat zal ik jullie nooit vergeten!

Graag wil ik de stafleden van de Pathologie bedanken voor jullie input, collaboratie en motivatie bedanken: Axel, (jetzt kann ich die Frage "na, schon promoviert?" endlich mit "ja" beantworten), Sylvia, Marion (speciaal voor de droge humor), Marjo en Jack.

Wat zou een lab zijn zonder analisten? Beste Erwin, Mat, Anique, Petra, Clairy, Danielle bedankt voor het soepel laten lopen van de dagelijkse lab werk en de technische expertise. 
I would like to thank Simon and Levy for your hard work and dedication during your internships which I had the pleasure to supervise.

Verder wil ik graag de Epi 's, Axels groep en rest van de medewerkers van de afdeling Pathologie bedanken.

Ook wil ik graag de medewerkers van de CPV voor het goede verzorgen van de muizen en de steun bij de logistiek van onze experimenten bedanken.

Dear lunch boys, Petri, small Antti, big Antti (aka the plumper) we shared some special moments not only during lunch. Dear Finland roomies, Erika and Line, thank you girls for the warm welcome, excellent companionship and introduction to the live in Finland. Dear Johanna, working with you was a great pleasure. The work we did for chapter 6 was amazing we started off from establishing a new technique while experimenting truly free. This curiosity driven process, developed interesting new ideas and was quite successful in the end.

Thanks you guys for the awesome time!

Research is not restricted to one department, I made many new friends at the department of biochemistry: Dennis, Martijn, Brecht, Armand, Rick, Tom and Leon. But also in the department of biomechanical engineering: Lauren and John and department of molecular genetics: Mike. And Tammy and Jasper of course. Thanks for the collaboration and the good times.

I would also like to thank my science friends and conference buddies from outside of the UM: dear Tom, Martin and Holger thanks for the good times!

Furthermore, I would like to thank all coauthors and collaborators who contributed to the research presented in this thesis.

I would like to thank the city of Maastricht and the Netherlands, as both have been my home for the past 9 years. I felt welcome here at all times and enjoyed every moment.

Danke liebe Rabenhaus Mitbewohner Merel, Chris, Horst, Stephan, Arno, Hanna unsere Zeit zusammen bleibt unvergesslich und lässt sich kaum in Worte fassen...

Liebe Kata, Hannah, Johanna, Anne, Benne, Matze Bu, Matze Lei, Maggi, Mareike mit euch würde ich jederzeit wieder studieren, ihr seid mega!

Liebe Mensa Chiller Dave, Dennis, Dima, Jo, Kevin (+ Caro), Marco, Nik, Steffen (RIP), Tristan ohne euch Jungs wäre es nicht dasselbe gewesen!

Dear Psycho-Boys and Casa-hangarounds Basti, Jonas and Dariusch, thank you guys for all the countless crazy nights back in the days! 
Dear Benno, you were my first friend in Maastricht and we sure did have a great time together. I appreciate that we still see each other and share our complete out of this world weirdo humor. To cite our fellow companion Erich Cartman: "I respect your authoritah" or thanks for your friendship and support during all these years.

Dear former Manos team-mates Sabrina, Fredo, Cons, Basti, Bernd, Bob, Simon, David, Wessel, Anika, Anika, Patrick, Timmi, Fabian, Justus, Viktor, Kaja, Topper thank you for the awesome time on the handball court and the adventures beyond!

Liebe Saskia und lieber Cristian, euch habe ich erst in meinen letzten Jahren in Maas kennengelernt aber mit euch würde ich jederzeit nach Portugal oder Düsseldorf auswandern um den Rest unseres Lebens Bacalao oder Blutwurst zu essen. Auf viele weitere Gönnungswochenenden!

Interesting times are approaching in Brussels and since Anna moved in 2010 I had the pleasure to get to know you Lea H, Lea P, Svenja, Bernd, Juliane, Amy, Becki and Daniel. Thank you guys for making me feel home already. I am looking forward to the awesome period ahead of us!

Dieses Dankwort wäre nicht komplett ohne mich bei den Wickrather Herren (und Damen) Bohnen, Strehlow, Volker, Julia, Fabian, Bodo, Maryam, Katrin und Vitus zu bedanken. Mit vielen von euch bin ich seit dem Kindergarten sehr eng befreundet, also eigentlich seitdem ich denken kann. Eine Freundschaft über so eine lange Zeit aufrecht zu erhalten ist außergewöhnlich und was auch passiert, auf euch kann und konnte ich mich immer verlassen. Herzlichsten Dank!

Lieber Wolfgang und liebe Monika, herzlichen Dank für eure lange Freundschaft und Unterstützung. Besonders in schwierigen Zeiten habt ihr mir und meiner Familie sehr geholfen und wart immer für uns da.

Liebe Birgit, danke für deine Unterstützung in den letzten 30 Jahren.

Lieber Markus, wir sind ziemlich unterschiedlich, haben verschiedene Ansichten und Interessen. Das hat in der Vergangenheit nicht immer Reibungslos funktioniert, aber wenn es drauf ankommt dann haben wir zusammen gehalten. Ich bin froh, dass du deine Bestimmung gefunden hast und mit Alex jemanden an deiner Seite der dich so gut ergänzt. Danke für die Unterstützung von euch beiden.

Liebe Mama, ich war sicherlich nicht das einfachste Kind und habe doch die ein oder andere Sorge bereitet. Du hast in all den Jahren immer hinter mir gestanden, egal was ich gemacht habe und mich in meinen Entscheidungen unterstützt und begleitet. Ohne deine Erziehung und Fürsorge wäre ich heute nicht der, der ich bin. Danke für all das!

Liebe Simo, lieber Andre, mit euch dem NRW Teil des Bergmann Paketes hat es natürlich sofort geklickt. Vielen Dank für euer offenes Ohr und eure Unterstützung vor allem in Karrierefragen. Ihr seid super Freunde! 
Liebe (erweiterte) Bergmanns Sophie, Jan, Tine, Benni und Johannes ich bin froh euch mit Anna zusammen in einem Pokeball bekommen zu haben, denn ohne euch wäre das Set einfach nicht komplett. Ihr seid inzwischen mehr als Familie und so unglaublich gute Freunde. Ich bin glücklich und dankbar für eure Unterstützung in den letzten Jahren!

Liebe Ute und lieber Christoph, bei euch wurde ich von Anfang an mit offenen Armen empfangen und habe mich sofort zu Hause gefühlt. Dazu kommt noch das wir zufällig recht viele gemeinsame Ansichten haben inklusive des Faibles für die Nordsee. Ich schätze eure Weltoffenheit, euer offenes Ohr und eure Meinung/Ratschläge auch in schwierigen Fragen. Ihr habt Anna und mich in der ganzen Zeit immer unterstützt, trotz der räumlichen Distanz, hierfür und für eure Freundschaft bin ich euch sehr dankbar!

Liebe Anna, mein größter Dank gilt dir. Ich habe beim Aufräumen der Materialien meiner zahlreichen gescheiterten Experimente, die es nicht in dieses Buch geschafft haben, noch Objektträger aus dem Jahr 2011 mit deiner Handschrift drauf gefunden. In jeder dieser kleinen Science-Krisen hast du immer mit angepackt und mich immer wieder aufgebaut. Ohne deine Unterstützung und deinen Rückhalt wäre dieses Buch niemals möglich gewesen. Es war eine schwierige Zeit, 5 Jahre konnten wir nicht zusammen wohnen, dazu anspruchsvolle Jobs, die Familie weit weg und unsere Freunde über ganz Europa verteilt. Das alles war nie ein ernsthaftes Problem, weil du dabei warst. Deine Offenheit schätze ich sehr an dir, sie hat dazu geführt das wir egal wo wir gelandet uns innerhalb kürzester Zeit wohlgefühlt haben und so viele gute Freunde gefunden haben. Langeweile kenne ich seitdem wir zusammen sind nicht mehr. Dein unvergleichlicher Humor macht jeden Moment unvergesslich. Die lange Zeit in der ich dich nicht regelmäßig sehen konnte, hat mich gelehrt jede Sekunde mit dir zu feiern und zu genießen. Ich weiß egal was auch kommt, zusammen können wir alles schaffen. Ich liebe dich! 


\section{Appendices}

Curriculum vitae

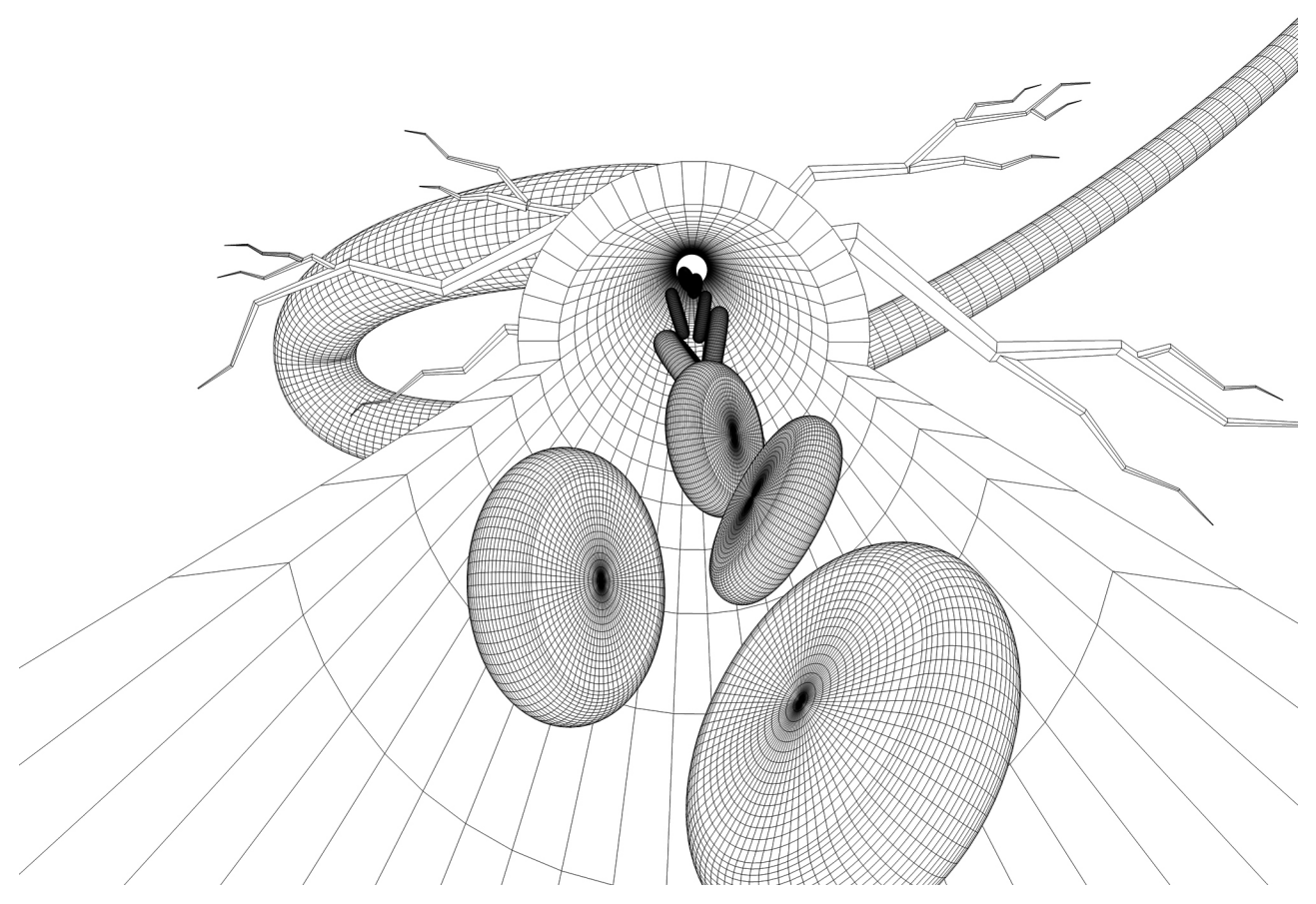


Name:

Date of birth:

Address:

Phone:

E-mail:

Research/Work Experience
Aug. 2012 - Jan. 2016

Nov. 2012 - Jun. 2012

Jul. 2011 - Nov. 2012

Feb. 2011 - Jul. 2011

Jul. $2010-$ Feb. 2011

Apr. 2010 - Jul. 2010
Thomas (Lukas) Theelen

29 October 1985 - Mönchengladbach, Germany

Overwinningsstraat 187A

1060 Brussels, Belgium

+32489840519

t.theelen@outlook.com
Service Design Consultant - Science Service Factory

(Maastricht University), the Netherlands

Optimizing the process of supplying medical

instruments to the hospitals in the European market

(Medtronic)

PhD training - Department of Pathology, Maastricht

University, the Netherlands

Promotors: Prof. Mat Daemen, Prof. Erik Biessen

Supervisor: Dr. Judith Sluimer

The Role of Hypoxia and Vascular Growth Factors in

Experimental Atherosclerosis

MSc Senior practical training - Department of

Biomedical Engineering, Maastricht University, the Netherlands

Project title: VE-cadherin as a potential mediator of microvascular permeability in atherosclerosis?

Student research assistant - Department of Pathology,

Maastricht University, the Netherlands

Project title: Carbogen oxygen treatment as a novel method to reduce plaque hypoxia and stimulate efferocytosis

MSc Junior practical training - Department of

Biotechnology and Molecular Medicine, University of Eastern Finland, Kuopio, Finland, A.I. Virtanen Institute Project title: The effects of periadventitial Ang-1/2 adenoviral gene transfer on the carotid arteries of atherosclerotic mice

Student research assistant - Department of Pathology, Maastricht University, the Netherlands

Project title: Molecular imaging of plaque hypoxia and angiogenesis using [18F]HX4 and [18F]RGD-K5

BSc Major practical training - Department of Pathology, Maastricht University, the Netherlands 
Project title: The effect of hypoxia on efferocytosis in the context of atherosclerosis

\section{Education}

Aug. 2012 - Jan. 2016

Sept. 2010 - Jul. 2012

Sept. $2007-$ Jul. 2010

Sept. 2004 - May 2006
PhD in Biomedical Sciences, Faculty of Health, Medicine and Life Sciences, Maastricht University, the Netherlands

MSc Clinical Molecular Sciences, Faculty of Health, Medicine and Life Sciences, Maastricht University, the Netherlands - graduated with distinction cum laude

BSc Biomedical Sciences, Faculty of Health, Medicine and Life Sciences, Maastricht University, the Netherlands

"Städt. Gesamtschule Hardt" (secondary school), Hardt, Germany

A-levels, biological profile

Additional Education

Jun. 2015

Apr. 2015

Feb. 2014

Nov. 2013

Feb. 2011

Jan. 2011

Jun. 2007
Summer school EIT Health Business and Innovation (Trinity College Dublin \& IESE Barcelona)

Advanced Scientific Writing Biomedical Sciences and Psychology (PhD-2)

Academic Writing for PhD Students (PhD-1)

Radiation protection level $5 b$ course

Safe microbiological techniques course

Laboratory animal science art. 9 course

Intensive summer course Dutch for Germans, Language Center Maastricht University, the Netherlands

Grants/Awards

Nov. 2014

Jul. 2014

Jul. 2014

Mar. 2013
Best Poster Award CARIM annual symposium, Maastricht, Netherlands

Best Poster Award Frontiers in Cardiovascular Biology, Barcelona, Spain

Travel grant European Society of Cardiology

Best Poster Award Winterschool, Tahko, Finland 
Mar. 2012 an research internship abroad

CARIM PhD Award 2012, Fellowship for four years of PhD training

Extra-curricular activities

Sept. 2010 - Sept. 2011

President of Student Handball Association MSHV Manos

Sept. 2009 -Sept. 2010

Treasurer of Student Handball Association MSHV Manos

$2004-2007$

Handball trainer at HSG Wickrath

Publications

1. Deficiency of the oxygen sensor prolyl hydroxylase 1 stimulates non-biliary cholesterol excretion attenuating hypercholesterolemia and atherosclerosis Marsch E, Demandt JA, Theelen TL, Tullemans BM, Boon MR, van Dijk TH, Gijbels MJ, Dubois LJ, Hung G, Fisher EA, Biessen EA, Daemen MJ, Rensen PC, Carmeliet P, Groen AK, Sluimer JC

Accepted for publication in Eur Heart J. (2016)

2. Quantification of endothelial $\alpha v \beta 3$ expression with high frequency ultrasound and targeted microbubbles: in vitro and in vivo studies.

Daeichin V, Bosch JG, Skachkov I, Kooiman K, Needles A, Theelen TL, Janssen B, Daemen MJ, van der Steen AF, de Jong N, Sluimer JC Accepted for publication in Ultrasound Med Biol. (2016)

3. The effect of prolonged dietary nitrate supplementation on atherosclerosis development Atherosclerosis Marsch E, Theelen TL, Janssen BJ, Briede JJ, Haenen GR, van Loon L, Poeze M, Bierau J, Gijbels MJ, Daemen MJ, Sluimer JC Atherosclerosis. 2015

4. Ablation of CD8 $\alpha+$ dendritic cell mediated cross-presentation does not impact atherosclerosis in hyperlipidemic mice Legein B, Janssen EM, Theelen TL, Gijbels MJ, Walraven J, Klarquist JS, Hennies CM, Wouters K, Seijkens TT, Wijnands E, Sluimer JC, Lutgens E, Zenke M, Hildner K, Biessen EA, Temmerman L.

Sci Rep. 2015

5. Deficiency of HIF1alpha in dendritic cells aggravates atherosclerosis and type $\mathbf{1} \mathbf{T}$ helper cell responses in mice 
Chaudhari SM*, Sluimer JC*, Koch M, Theelen TL, Manthey HD, Busch M, CaballeroFranco C, Vogel F, Cochain C, Pelisek J, Daemen MJ, Lutz MB, Görlach A, Kissler S, Hermanns HM, Zernecke A.

Arterioscler Thromb Vasc Biol. 2015

6. Angiopoietin-2 blocking antibodies reduce early atherosclerotic plaque development in mice.

Theelen TL*, Lappalainen JP*, Sluimer JC*, Gurzeler E, Cleutjens JP, Gijbels MJ, Biessen EA, Daemen MJ, Alitalo K, Ylä-Herttuala S.

Atherosclerosis. 2015

7. Reversal of hypoxia in murine atherosclerosis prevents necrotic core expansion by enhancing efferocytosis.

Marsch $E^{*}$, Theelen $T L^{*}$, Demandt JA, Jeurissen $M$, van Gink $M$, Verjans $R$, Janssen $A$, Cleutjens JP, Meex SJ, Donners MM, Haenen GR, Schalkwijk CG, Dubois L, Lambin P, Mallat Z, Gijbels MJ, Heemskerk JW, Fisher EA, Biessen EA, Janssen BJ, Daemen MJ, Sluimer JC.

Arterioscler Thromb Vasc Biol. 2014

8. Increased adventitial lymphatic capillaries in atherosclerosis: a path for $\mathbf{T}$ cell trafficking?

Rademakers T, van der Vorst EP, Daissormont IT, Otten JT, Theelen TL, Gijbels MJ, Anisimov A, Nurmi H, Zandvoort MA, Schober A, Heeneman S, Alitalo K, Biessen EA Submitted

9. Effect of remote ischemic preconditioning on the release kinetics of high-sensitivity cardiac troponin I and $\mathrm{T}$ following a $\mathbf{3 0} \mathrm{km}$ run: a randomized controlled crossover study

Klinkenberg LJ, Luyten P, van der Linden N, Urgel K, Snijders DP, Knackstedt C, Dennert R, Kietselaer BL, Mingels AM, Cardinaels EP, Peeters FE, van Suijlen JD, ten Kate J, Marsch E, Theelen TL, Sluimer JC, Wouters K, Bekers O, Bekkers BC, van Loon $\amalg$, van Dieijen-Visser MP, Meex SJ

Submitted

10. Histamine and VEGF mediate pro-angiogenic gene expression and endothelial cell junction formation through common cellular regulators

Laakkonen JP*, Lappalainen JP*, Theelen TL, Toivanen PI, Nieminen T, Kaikkonen MU, Sluimer JC, Ylä-Herttuala S

Submitted

11. Myeloid prolyl hydroxylase domain-containing protein 2 deficiency leads to larger, but more stable atherosclerotic plaques in mice

Theelen TL, Marsch E, Demandt JA, Fallais S, Tullemans BM, van der Vorst EP, Donners MM, Gijbels MJ, Welting TJ, Reutelingsperger CP, Mastenbroek TG, Cosemans JM, Willems BA, Schurgers LJ, Carmeliet P, Biessen EA, Daemen MJ, Sluimer JC

Submitted 
1. Myeloid PHD2 deficiency impairs macrophage collagen degradation stimulating atherosclerotic plaque stability in mice

Theelen TL, Marsch E, Demandt J, Fallais S, van der Vorst E, Dinjens C, Gijbels ML, Welting TJM, Mastenbroek T, Cosemans JM, Willems BAG, Schurgers LJ, Biessen E, Carmeliet P, Daemen MJAP, Sluimer JC. Gordon Research Conference Atherosclerosis 2015, Newry, USA - Poster presentation

2. Myeloid PHD2 deficiency impairs macrophage collagen degradation resulting in enlarged and fibrotic atherosclerotic plaques in mice

Thomas L Theelen, Elke Marsch, Jasper AF Demandt, Emiel van der Vorst, Clairy Dinjens, Marion L Gijbels, Tim JM Welting, Tom Mastenbroek, Judith M Cosemans, Brecht AG Willems, Leon J Schurgers, Erik Biessen, Peter Carmeliet, Mat JAP Daemen, Judith C Sluimer. International Symposium on atherosclerosis 2015, Amsterdam, the Netherlands - Guided poster presentation

3. Myeloid PHD-2 deficiency leads to enlarged and fibrotic atherosclerotic plaques in mice

Theelen TL, Marsch E, Demandt JAF, Van Der Vorst EP, Welting TJM, Willems BAG, Schurgers LJ, Dinjens C, Gijbels MJ, Carmeliet P, Daemen MJAP, Sluimer JC. CARIM annual symposium 2014, Maastricht, the Netherlands - Poster presentation (awarded best poster)

4. Myeloid PHD-2 deficiency leads to enlarged and fibrotic atherosclerotic plaques in mice

Theelen TL, Marsch E, Demandt JAF, Van Der Vorst EP, Dinjens C, Gijbels MJ, Carmeliet $\mathrm{P}$, Daemen MJAP, Sluimer JC. Dutch Endothelial Biology Society Meeting 2014, Biezenmortel, the Netherlands - Oral presentation

5. Myeloid PHD-2 deficiency leads to enlarged and fibrotic atherosclerotic plaques in mice

Theelen TL, Marsch E, Demandt JAF, Van Der Vorst EP, Dinjens C, Gijbels MJ, Carmeliet P, Daemen MJAP, Sluimer JC. Cardiovascular PHD course 2014, Papendal, the Netherlands - Poster presentation

6. Myeloid PHD-2 deficiency leads to enlarged and fibrotic atherosclerotic plaques in mice

Thomas L Theelen, Elke Marsch, Jasper Demandt, Clairy Dinjens, Jack Cleutjens, Marion Gijbels, Peter Carmeliet, Mat Daemen, Judith Sluimer. Fontiers in Cardiovascular Biology 2014, Barcelona, Spain - Poster presentation (awarded as best poster)

7. Myeloid PHD-2 deficiency leads to enlarged and fibrotic atherosclerotic plaques in mice

Thomas L Theelen, Elke Marsch, Jasper Demandt, Clairy Dinjens, Jack Cleutjens, Marion Gijbels, Peter Carmeliet, Mat Daemen, Judith Sluimer. $5^{\text {th }}$ International Meeting on Angiogenesis 2014, Amsterdam, the Netherlands - Poster presentation

8. Myeloid PHD-2 deficiency leads to enlarged and fibrotic atherosclerotic plaques in mice 
Thomas L Theelen, Elke Marsch, Jasper Demandt, Clairy Dinjens, Jack Cleutjens, Marion Gijbels, Peter Carmeliet, Mat Daemen, Judith Sluimer - Cardiovascular conference (CVC) 2014, Ermelo, the Netherlands - Poster presentation

9. VE-cadherin as a potential mediator of microvascular permeability in atherosclerosis?

Thomas L. Theelen, Timo Rademakers, Jaap D. van Buul, Jack P. Cleutjens, Pieter van de Vijver, Tilman M. Hackeng, Marc A.M.J. van Zandvoort, Mat J.A.P. Daemen, Judith C. Sluimer. European Society of Cardiology summer school, Nice, France-Poster presentation

10. VE-cadherin as a potential mediator of microvascular permeability in atherosclerosis?

Thomas L Theelen, Timo Rademakers, Jaap van Buul, Jack Cleutjens, Pieter van de Vijver, Tilman Hackeng, Marc van Zandvoort, Mat Daemen, Judith Sluimer. Focus on Microscopy 2013, Maastricht, The Netherlands - Poster presentation

11. Seek the leak - Measuring vascular permeability using in vivo multi-photon laser scanning microscopy Thomas L Theelen, Jari Lappalainen, Johanna Laakkonen, Judith Sluimer, Seppo Ylä-Herttuala. The $7^{\text {th }}$ annual post-graduate symposium of the doctoral program in molecular medicine 2013 (University of Eastern Finland), Tahko, Finland - Poster presentation (awarded as best poster)

12. Small vessels with huge impact: leaky microvessels exacerbate atherosclerosis. Thomas L Theelen Annual Symposium 2012 of Cardiovascular Research Institute Maastricht (CARIM), Maastricht, the Netherlands - Oral presentation

13. Small vessels with huge impact: leaky microvessels exacerbate atherosclerosis. Thomas L Theelen $4^{\text {th }}$ International Life Sciences Master Research Conference 2012 at Hasselt University, Hasselt, Belgium - Oral presentation

14. Periadventitial adenoviral Ang-1/2 gene transfer interferes with atherosclerotic plaque progression and angiogenesis in the carotid artery of Idlr ${ }^{-/-}$apob ${ }^{100 / 100}$ mice. Thomas L Theelen, Jari Lappalainen, Fons Verheyen, Erik Biessen, Einari Aavik, Kari Alitalo, Mat Daemen, Judith Sluimer, Seppo Ylä-Herttuala. Cardiovascular conference (CVC) 2012, Leeuwenhorst, the Netherlands - Oral presentation

15. The effects of a periadventitial adenoviral Ang-1 gene transfer on the carotid arteries of atherosclerotic mice.

Thomas L Theelen, Jari Lappalainen, Einari Aavik, Fons Verheyen, Mat Daemen, Judith Sluimer, Seppo Ylä-Herttuala. The $8^{\text {th }}$ International Duodecim Symposium, Endothelial Growth Factors In Cancer and Cardiovascular Diseases 2011, Vanajanlinna, Finland Poster Presentation 